\title{
Electrical Percolation Threshold of Magnetostrictive Inclusions in Piezo-Electric Matrix Composite as a Function of Relative Particle
} Size

Antoine Joseph Bedard Jr.

Follow this and additional works at: https://researchrepository.wvu.edu/etd

\section{Recommended Citation}

Bedard Jr., Antoine Joseph, "Electrical Percolation Threshold of Magnetostrictive Inclusions in PiezoElectric Matrix Composite as a Function of Relative Particle Size" (2017). Graduate Theses, Dissertations, and Problem Reports. 5173.

https://researchrepository.wvu.edu/etd/5173

This Dissertation is protected by copyright and/or related rights. It has been brought to you by the The Research Repository @ WVU with permission from the rights-holder(s). You are free to use this Dissertation in any way that is permitted by the copyright and related rights legislation that applies to your use. For other uses you must obtain permission from the rights-holder(s) directly, unless additional rights are indicated by a Creative Commons license in the record and/ or on the work itself. This Dissertation has been accepted for inclusion in WVU Graduate Theses, Dissertations, and Problem Reports collection by an authorized administrator of The Research Repository @ WVU.

For more information, please contact researchrepository@mail.wvu.edu. 


\title{
Electrical percolation threshold of magnetostrictive inclusions in piezo-electric matrix composite as a function of relative particle size
}

\author{
Antoine Joseph Bedard Jr. \\ Dissertation submitted \\ to the College of Engineering and Mineral Resources \\ at West Virginia University \\ in partial fulfillment of the requirements for the degree of \\ Doctor of Philosophy in \\ Mechanical Engineering \\ Ever J. Barbero, Ph.D., Chair \\ Fritz A. Campo, Ph.D., \\ Xueyan Song, Ph.D., \\ Yun Chen, Ph.D., \\ Eduardo M. Sosa, Ph.D \\ Department of Mechanical and Aerospace Engineering \\ Morgantown, West Virginia \\ 2017
}

Key words: particle dynamics, magnetostrictive, piezoelectric, magnetoelectric, percolation, LAMMPS (C) Copyright 2017 Antoine Joseph Bedard Jr. 


\begin{abstract}
Magnetoelectric (ME) composites can be produced by placing magnetostrictive particles in a piezoelectric-matrix. Ferrite magnetostrictive $(\mathbf{H})$ particles, if allowed to percolate, can short the potential difference generated in the piezoelectric (E) phase. This work focuses on modeling an $\mathbf{M E}$ composite as bi-disperse hard shells with the magnetostrictive $\mathbf{H}$ particles scaled to $100 \mathrm{~nm}$ where particle dynamics is used to explore relationships among relative particle size, particle affinity, and electrical percolation with the goal of maximizing the percolation threshold. It was found that the two factors that increase the $\mathbf{H}$ to $\mathbf{H}$ intra-phase percolation threshold are: (1) the size of $\mathbf{H}$ particles relative to the $\mathbf{E}$ particles, and (2) the affinity between the $\mathbf{H}$ and $\mathbf{E}$ particles. Other factors that were also found to decrease the same percolation threshold are: the (1) deformation of the $\mathbf{H}$ particles from spherical geometry, and (2) a tipping point in the relative $\mathrm{R}_{\mathrm{H}} / \mathrm{R}_{\mathrm{E}}$ size ratio where if the size of the $\mathbf{E}$ particles goes below a value of approximately (3.5) ${ }^{-1} \mathbf{H}$ particle size, then the percolation threshold decreases.
\end{abstract}




\section{Acknowledgements}

I would like to sincerely thank my dissertation committee and my advisor Dr. Ever Barbaro without whose help with fleshing out details as well as overall guidance and direction this work would not have been possible.

I would also like to thank my prior supervisors and advisors Dr. W. Chu and Mr. M. Goede, Dr. Glenn Dalrymple (retired from the University of Nebraska Medical Center), and coworkers Ralph Bunting and Becky Edwards also formerly with the Nebraska Medical Center. In addition, I would like to thank Dr. Mesfin Tsige currently with the University of Akron Department of Polymer Science, Dr. John McCoy with the New Mexico Institute of Mining and Technology, and Dr. Estela Blaisten-Barojas with George Mason University Center for Simulation and Modeling, all of whom contributed to my professional growth and understanding of science.

Lastly, I would like to thank my parents Antoine Joseph Bedard Sr. and Linda C. Bedard for their love, and my friends Katya Bray, John Gage, Patrick and Julie Dwyer, Rich Raiders, and Kathy Huelsing for their encouragement and friendship over the years. 


\section{Table of Contents}

I. Acknowledgements iii

II. Table of Contents $\quad$ iv

Chapter 1: Literature Review

1. Introduction 1

2. Problems that diminish the ME effect 2

3. Polydispersity 3

4. Ramdom close packed particle configuration 3

5. Percolation 4

6. Segregation $\quad 5$

7. Glass transition $\quad 5$

$\begin{array}{ll}\text { 8. Particle affinity } & 6\end{array}$

$\begin{array}{ll}\text { 9. References } & 7\end{array}$

Chapter 2: Electrical percolation threshold of magnetostrictive inclusions in a piezoelectric matrix composite as a function of relative particle size

Abstract

$\begin{array}{ll}\text { 1. Introduction and Objectives } & 14\end{array}$

2. Methodolgy 16

$\begin{array}{ll}\text { 3. Results } & 24\end{array}$

4. Analysis 31 
Chapter 3: Electrical percolation threshold of magnetostrictive inclusions in a piezoelectric matrix under simulated sintering conditions

\section{$\underline{\text { Page }}$}

Abstract 40

1. Introduction and Objectives 40

2. Methodolgy 43

3. Three Dimensional Particle Dynamics 45

4. Characterizations and Measurment 51

5. Results 53

6. Conclusions 63

7. References 65

Chapter 4: Extension of hard-shell methods from Chapter 2 applied to relative size $R_{H} / R_{E}=1,2,3,4$

1. Introduction

2. Review of chapter 2

3. Methods and results for $\mathrm{R}_{\mathrm{H}} / \mathrm{R}_{\mathrm{E}}=4$

4. Analysis

5. References 
Chapter 5: Conclusions and future work

$\begin{array}{lr}\text { 1. Conclusions } & 80\end{array}$

2. Future work $\quad 82$

3. References $\quad 83$

Appendix A. Programs for data processing of hard-shell results per chapter $2 \quad 85$

Appendix B. Programs for processing particle deformation per chapter $3 \quad 106$

Appendix C. Input LAMMPS (C Scripts used for particle simulations 129 


\section{Chapter 1}

\section{Literature Review}

\section{Introduction.}

1.1 Magnetoelectric materials.

Multiphase magnetoelectric materials (ME) are composed of piezoelectric (E) and magnetostrictive (H) components [1-9] [20] [52-53]. Piezoelectric materials are those for which an electric polarization occurs as a result of an applied stress [1-3]. Magnetostriction is the inducing of magnetic polarization in response to an applied mechanical stress [1]. When these two materials are combined, they form a magnetoelectric two-phase composite, which, via coupling of mechanical strain in common with both materials, produces a resultant material whereby an electrical polarization occurs in response to an applied mechanical field or vise-versa [3]. The ME effect was first discovered to occur naturally, albeit weakly, in 1888 by Wilhelm C. Rontgen [9], and in 1972, J. van Suchtelen first proposed the ME concept for artificially produced ME composites [3]. In 2000 N.Hill [1] discussed conditions for ferroelectricty and ferromagnetism to be compatible with oxides, ushering in a wave of experimental research in response. Multiferric magnetoelectric technology finds application in sensors, transducers, oscillators, energy harvesters, phase shifters, and multiple state memory devices and spintronics (electronics utilizing electron spin) [3-4].

1.2 Molecular dynamics.

Molecular dynamics has been used to model hard-shell granular systems [54] [55] [31-33] [56] [48] [57] [58], and in particular the Leonard-Jones potential has been used to model granular materials [31-32] [59-62]. Bell et al. [59] report results using molecular dynamics soft-sphere methods which are a common way to simulate granular materials such as spheres and polyhedrals. Foffi et al. [31] reports molecular dynamics simulation results for binary mixtures of hard spheres for different size disparities 
and mixing percentages. Zaccarelli et al. [48] report event-driven molecular dynamics simulations of particles interacting via a maximum valence model.

2. Problems that diminish the ME effect.

2.1 Manufacture.

There are problems inherent with the manufacture of ME composite materials. One problem is atomic diffusion during the nucleation growth process [8] [14-17]. Another problem is chemical reactions during manufacture between the component materials and thermal expansion mismatch which diminishes the ME effect [3] [8-9].

2.2 ME mechanical coupling versus segregation

To maximize the ME effect, it is essential that the materials be thoroughly mixed to maximize mechanical contact and thus maximize the $\mathbf{M E}$ coupling coefficient between the two phases $\mathbf{H}$ and $\mathbf{E}$ [8] [14-17]. However, when the particles are mixed, often times as a function of relative size, density, and velocity differences, granular particles will segregate or show dynamic heterogeneities [27] [32] [37] [42] [50] [62-63]. This segregation causes “demixing” which reduces the mechanical contact between the $\mathbf{H}$ and $\mathbf{E}$ phases [32] [50] [63-64].

2.3 Quantum tunneling and percolation.

Because the $\mathbf{H}$ phase is generally conductive, it is also critical that the $\mathbf{H}$ phase does not percolate or form a connective chain across the material [14-17]. If the $\mathbf{H}$ particles do percolate, then the piezoelectric charge separation or voltage of the $\mathbf{E}$ phase will be shorted or reduced, thus diminishing the overall ME effect [1] [14-17]. In addition, even if the $\mathbf{H}$ particles are not physically connected, there is the problem of quantum tunneling [12] [22]. Quantum tunneling between adjacent $\mathbf{H}$ particles is a quantum mechanical effect whereby charge transport or collective plasma resonance between neighboring particles can occur between classically insulated adjacent particles [12] [22]. Numerous researchers [2124] [27] [63] have described the phenomena of charge transfer between granular and granular conducting particles. A good reference on the fundamentals of quantum tunneling as it applies to chemical reactions 
among particles can be found in [21]. Beloborodov et al. [22] investigate the Coulomb interaction and hopping transport in granular metals where they develop a theory of tunneling through chains of granular boundaries.

\section{Polydispersity}

Relative particle size or polydispersity affects the maximum packing fraction than can be obtained for random close packed configuration of hard spheres [19] [34] [35] [58] [62-66]. Polydispersity increases the volume fraction of the random close packed hard shell particle configuration due to the smaller spheres filling in the interstitial spaces between larger spheres [57], thus decreasing the packing fraction. Kuzy [19] in particular developed a model whereby the surface fraction of the primary phase is covered by the dispersed secondary phase. Titscher and Uncer [54] report a method whereby the problem of polydisperse sphere packing is applied to concrete with a molecular dynamics simulation using growing particles. They found a minimum distance between particles influences that characterizes percolation. This minimum distance they interpret as a thin film surrounding each particle, and this minimum distance may depend on the volume fraction [54].

\section{Random close packed particle configuration}

Random close packed systems are amorphous packed systems for which the maximum packing fraction is obtained but no crystal structural is apparent [33-36] [55-58] [67]. For close packed systems of equally sized or monodisperse spheres, the maximum packing fraction is $f=0.64$ [55-56] [67]. Kansal et al. out of Princeton University [35] report on a method to generate dense polydisperese sphere packing via slowly growing the spheres until a maximum packing fraction is reached, and they report a range of maximum packing fractions for bidisperese spheres is reported to range from 0.64 (equally sized spheres) to 0.78 when radius of the smaller particles is smaller than the large by a factor of 10 or more. Donev et al. [55], also out of Princeton University (Prinston New Jersey), using a hard-sphere molecular dynamics in which spheres are grown until a final state with diverging collision rate is reach report the event of 
jamming (immobility) for hard spheres with a packing fraction $\mathbf{f}=0.64$, and they report amorphous bidsiperse disk were jammed at a packing fraction $\mathbf{f}=0.84$. Numerous other investigators [19] [37] [54] [68-71] have developed models which consider the volume fraction requirements necessary to form a percolative network as a function of particle size ratio and packing fraction for random close packed particle configurations.

\section{Percolation}

Percolation Theory was developed to address disordered media or variations in the degree of connectivity [51] [72-73]. A connectivity search among particles or nodes may be performed both iteratively [51] [73] or (if interpreted in terms of graphs and tree traversal) via recursion [40]. Percolation may be defined as a phase transition for which a significant macroscopic change occurs as a result of connectivity among a subset of particles [37]. The percolation threshold is the critical point corresponding to the onset of long-range connectivity [12] [74]. In order for ME materials to function, percolation or connective paths between $\mathbf{H}$ particles must be minimized, which is accomplished by maximizing the percolation threshold [8] [12] [37]. For equalized monodisperse spheres, the percolation threshold or critical volume fraction ( $\boldsymbol{\rho})$ is 0.16, the Scher-Zallen invariant [12] [18] [72]. The percolation threshold $(\boldsymbol{\rho}=0.16)$ and the packing fraction $(\mathbf{f}=0.64)$ are related by the equation $\boldsymbol{\rho}=\mathbf{f} \times \mathbf{p}$ where $\mathbf{p}$ is the critical percolation probability [18]. E. Garboczi and D. Benz of U.S. National Institute of Standards and Technology (NIST) [68-69] in their extensive report show how the multi-length scale and random microstructure of concrete can be interpreted with percolation theory. For example, Garboczi and Benz [68] report that of degrees of hydration of $1.8 \%, 2.7 \%$, and $4.6 \%$ are required to achieve percolation when the water to concrete ratios are $0.3,0.4$ and 0.5 respectively, where the degree of hydration is the volume fraction of cement that has reacted with water. Grunlan et.al [37] report the lowering of percolation thresholds of carbon black-polymer composites, where particles are forced into conductive pathways at low concentrations because of their inability to occupy volume claimed by larger particles. Zhu et al. [75] report the effects of different particle aspect ratios and polydisperse particle size 
differences on percolation in heterogeneous composites, where percolation transitions were observed in the context of thermal conductivity. Schilling et al. [44] review the effects that particle anisotropy (deviations from spherical particle shape) and changes in volume fraction have on the percolation thresholds of colloidal suspension ranging in size from 10 to 10,000 nm. Lamas et al. [71] report the main factors governing thermal conductivity hence percolation for carbon nanotubes suspended in fluid are size, shape and particle aspect ratio, and that the Brownian motion and structural flexibility of the nanotubes have a negligible effect on overall conductivity.

\section{Segregation}

The phenomenon of segregation is where differences in size or density or velocity cause populations of particles to separate or become isolated from each other [32] [42] [50] [62-63]. Researchers [32] [63] consider the effects of the difference in size on the segregation of granular materials. Puertas et al. [47] describes dynamical heterogeneities within the context of particle dynamics of a percolating network. Vaart et al. [42] report that large and small particles show an asymmetry as a function of local particle concentration and differences in particle dynamics. Rapaport [62] gives a concise overview of counterintuitive granular segregation particle dynamics involving molecular dynamics modeling involving dependencies on volume fraction and damping.

\section{Glass transition}

Glass transition is described as a sharp change from a viscous, rubbery, or fluid state to solid or gel state [31] [33] [48] [67]. Specifically, [67] defines a glass transition for random close packed and loose packed hard shell systems as a precipitous change in a macroscopic property such as diffusion. At high packing fractions, arrest of particle motion can take place via a glass transition which can be driven by jamming of hard-sphere systems [48]. Donev et al. [55] report testing form jamming condition of particle random packing. Parisi et al. [33] review applications of mean field theory to hard sphere jamming where in three dimensions they obtain expressions for the radial distribution functions close to jamming, as well 
as an equations of state for glass for slow compression rates, and for binary mixtures, the variation with mixture composition of the jamming density and partial average number of contact points or coordination numbers.

8. Particle affinity.

\subsection{Sintering}

Sintering is the process of bonding particles of a powder by heat and pressure without liquefaction [8] [12] [14-17]. Sintering can have an effect on the ME coupling coefficient [8]. Bichurin et al. [4] state their methods of producing magnetoelectric composites capture aspect of ME design using sintering methods. de Brito et al. [5] report sintering temperatures for CFO ferrites between 1000 to 1500 degrees Celsius, and report in the context of ME transducers the use of chemical sintering additives than form a liquid during sintering as an alternative to lowering the sintering temperature for ceramics. Sharif et al. [76] report the microstructure and electrical properties of PZT ceramics are highly sensitive to sintering conditions.

\subsection{Functionalization}

Nappini et al. [41] report methods and efficacy to induce surface charges and bonding sites on magnetic cobalt ferrite CFO nanoparticles, and how, when properly functionalized with organic molecules, represent a promising approach to biomedical applications such as magnetic resonance imaging and drug delivery. 


\section{References}

1. W. Eerenstein, N.D. Mathur, J.F. Scott (2006) Multiferroic and magnetoelectric materials. Nature, 442(17):759-765

2. R. Grossinger, G.V. Duong, R. Sato-Turtlli (2008) The physics of magnetoelectric composites. Journal of Magnetism and Magnetic Materials, 320:1972-1977

3. J. Ma, J. Hu, Z. Li, C-W. Nan (2011) Recent progress in multiferric magnetoelectric composites: from bulk to thin films. Advanced Matter, 23:1062-1087

4. M.Bichurin, V.Petrov, S.Priya, A.Bhalla (2012) Editorial multiferric magnetoelectric composites and their applications. Advances in Condensed Matter Physics, 2012(129794):1-3

5. V.L.O. de Brito, S.A. Cunha, L.V. Lemos, C.B. Nunes (2012) Magnetic properties of liquidphase sintered $\mathrm{CoFe}_{2} \mathrm{O}_{4}$ for application in magneoelastic and magnetoelectric transducers. Sensors, 12:10086-10096

6. R. Ramesh, N.A. Spaldin (2007) Multiferrics: progress and prospects in thin films. Nature Matrials, 6:21-29

7. Y-H. Chu, L.W. Martin, M.B. Holcomb, M. Gajek, S-J.Han, Q. He, N. Balke, C-H. Yang, D. Lee, W. Hu, Q. Zhan, P-L. Yang, A. Fraile-Rodriquez, A. Scholl, S.X. Wang, R. Ramesh (2008) Electric-field control of local ferromagnetism using magnetoelectric multiferric. Nature Materials, 7:478-482

8. C-W. Nan, M.I. Bichurin, S. Dong, D. Veichland, G. Srinivasan (2008) Multiferric magnetoelectric composites: historical perspective, status, and future directions. J. Appl. Phys, 103:031101

9. N. Ortega, A. Kumar, J.F. Scott, R.S. Katlyar (2015) Multifunctional magnetostatic materials for device applications. J. Phys: Concens. Matter, 27:504002 
10. N.S.A.B. Sharif (May 2015) Synthesis and characterization of lead zirconate titanate $\left(\mathrm{Pb}\left[\mathrm{Zr}_{0.52} \mathrm{Ti}_{0.48}\right] \mathrm{O}_{3}\right)$ properties via high energy planetary ball milling. Ph.D. dissertation, Manufacturing Engineering, University Malaysia Pahang

11. C.A. Randall, N. Kim, J-P. Kucera, W. Cao, T.R. Shrout (1998) Intrinsic and extrinsic size effects in fine grained morphotropic-phase-boundary lead zirconate titanete ceramics. J. Am. Ceram, 81(3):677-688

12. C-W. Nan, Y. Shen, J. Ma (2010) Physical properties of composites Near Percolation. Annual Review of Materials Research, 40:131-51

13. A. Bunde, W. Deiterich (2000) Percolation in composites. Journal of Electroceramics, 5(2):81-92

14. T.I Muchenic, E.J. Barbero ( 13-16 October 2014) Micromechanics modeling of magnetoelectric Composites. The Composites and Advanced Materials Expo. (CAMX ) Conference Proceedings, Orlando FL

15. T.I.Muchenik, E.J.Barbero (2015) Charge, voltage and work-conversion formulas for Magnetoelectric laminated composites. Smart Mater and Structure, 24:025039

16. T.I. Muchenik (2016) Modeling of magnetoelectric composite structures. Ph.D. dissertation, West Virginia University

17. T.I. Muchenik, E.J. Barbero (2016) Prediction of extrinsic charge, voltage, and work-conversion factors for laminated Magnetoelectric composites. Smart Mater and Structure, 25:015006.

18. H. Scher, R. Zallen (1970) Crtical Density in Percolation Processes. J. Chem. Phys., 53:37593761

19. R.P. Kusy (1977) Influence of particle size ratio on the continuity of aggregates. Journal of Applied Physics, 48:5301

20. K.S. Deepa, S.K. Nisha, P. Parameswaran, M.T. Sebastian, J. James (2009) Effect of conductivity on filer on the percolation threshold of composites. Applied Physics Letters, 94:142902

21. D.Carrera (28 Nov 2007) Quantum tunneling in chemical reactions, MacMillan Group Meeting. https://www.princeton.edu/chemistry/macmillan/group-meetings/DEC_tunneling.pdf 
22. I.S. Beloborodov, A.V. Lopatin, V.M. Vinokur (2005) Coulomb effects and hopping transport in granular metals. Physical Review B, 72:125121

23. R.T. Hill, J.J. Mock, S.D. Wolter, N.M.Jokest, D.R. Smith, A. Chilkoti (2012) Plasmon ruler with angstrom length resolution. ACS NANO, 6(10):9237-9246

24. S. Kadkhodazadeh, J.B. Wagner, H. Kneipp, K. Kneipp (2013) Coexistance of classical and quantum plasmonics in large plasmonic structures with subnanometer gaps. Applied Physics Letters, 103:083103.

25. J.A. Scholl, A. Garcia-Etxarri, A.L. Koh, J.A. Dionne (2013) Observations of quantum tunneling between two plasmonic nanopoles. American Chemical Society Publications, Nano Letter, 13:564-569

26. J. Zhang, B.I. Shklovskii (2004) Density of states and conductivity of a granular metal or array of quantum dots. Physical Review B, Vol.70:153317

27. J.Li, J-K. Kim (2007) Percolation threshold of conducting polymer composites containing 3D randomly distributed Graphite Nanoplatlets. Composites Science and Technology, 67:2114-2120.

28. X. Zhu (1 Dec 2013) Tutorial on Hertz Contact Stress. OPTI 512, https://wp.optics.arizona.edu/optomech/wp-content/uploads/sites/53/2016/10/OPTI-521-Tutorialon-Hertz-contact-stress-Xiaoyin-Zhu.pdf

29. V. Popov (2010) Contact Mechanics and Friction. Springer-Verlag Berlin Heidelberg, file://C:/cygwin/home/WVU/DISSR/R_Barbero/Papers/Springer.Contact.Mechanics.And.Frictio n.Mar.2010.eBook-ELOHiM.pdf

30. S. J. Plimpton (1995) Fast parallel Algorithms for short-range molecular dynamics. J. Comp. Phys., 117:1-19

31. G. Foffi, W. Goetz, F. Sciortino, P. Tartaglia, T. Voigtmann (2003) Mixing Effects for the Structural Relaxation in Binary Hard-Sphere Liquids. Physical Review Letters, 91(8):085701

32. J.M. Ottino, D.V. Khakhar (2000) Mixing and Segregation of Granular Materials. Annual Review Fluid Mechanics, 32:55-91 
33. G. Parisi, F. Zamponi (2010) Mean Field Theory of Hard Sphere Glasses and Jamming. Condensed Matter, Rev. Mod. Phys. 82:789

34. H.J.H. Brouwers (2006) Particle-size Distribution and Packing Fraction of Geometric Random Packing. Physical Review E, 74:031309

35. A.R. Kansal, S. Torquato, F.H. Stillinger (2002) Computer Generation of Dense Polydisperse Sphere Packing. Journal of Chemical Physics, 117:8212

36. G.D.Scott, D.M. Kilgour (1969) The Density of Random Close Packing of Spheres. British Journal Applied Physics, 2(2):863-866

37. J.C. GRunlan, W.W. Gerberich, L.F. Francis (2001) Lowering the percolation threshold of conductive composites using particulate polymer microstructure. Journal of Applied Polymer Science, 80:69-705

38. H.M.Hasanabadi, M. Wilhelm, D. Rodrigue (2014) A rheological criterion to determine the percolation threshold in polymer nano-composites. Rheol. Acta, 53:869-882

39. R. Eisberg, R.Resnick (1985) Quantum Mechanics of Atoms, Molecules, Solids, Nuclei and Particles. John Wiley and Sons, $2^{\text {nd }}$ Ed

40. R.L. Kruse (1989) Programming with data structures. Chap.17.3 Graph Traversal, Prentice Hall

41. S. Nappini, E. Magnano (2015) Surface Charge and Coating of $\mathrm{CeFe}_{2} \mathrm{O}_{4}$ Nanoparticles: Evidence of Preserved Magnetic and Electronic Properities. J. Phys. Chem C, 119:25529-25541.

42. K.van der Vaart, P. Gajjar, G. Epely-Chauvin, N. Andreini, J.M.N.T. Gray, C. Ancey (2015) An underlying asymmetry within particle-size segregation. Phys. Rev. Let., 114:238001

43. C.P. Royall, S.R. Williams, T. Ohtsuka, J. Tanaka (2008) Direct observation of a local structural mechanism for dynamic arrest. Nature Materials, 7:556-561

44. T. Schilling, S. Dorosz, M. Radu, M. Mathue, S. Jungblut, K. Binder (2013) Mixtures of qnsiotropic and spherical colloids: phase behavior, confinement, percolation phenomena and kinetics. Eur. Phys. J. Special Topics, 222:3039-3052 
45. M-A. Suarez, N. Kem, W. Kob (2009) Out-of-equilibrium dynamcis of a fractal model Gel. J. Chem. Phys., 130: 194904

46. A.Amirjanov, K. Sobolev (2008) Optimization of a computer simulation model for packing of concrete aggregates. Particulate Science and Technology, 26 (4):380-39

47. A.M. Puertas, M. Fuchs, M.E. Cates (2004) Dynamical heterogeneities close to a colloidal gel. J. Chem. Phys., 121(6):2813-2822

48. E. Zaccarelli, S.V. Buldyrev, E. La Nave, A.J. Morene, I. Saika-Voivod, F. Sciortino, P. Tartaglia (2005) Model for reversible colloidal gelation. Physical Review Letters, 94:218301

49. G.S.Rohr (2001) Structure and bonding in crystalline materials. Cambridge University Press.

50. M. Nakagawa, J.L. Moss, S.A. Altobelli (1999) MRI measurements and granular dynamics simulation of segregation of granular mixture. Proceedings of forth microgravity fluid physics and transport phenomena (NASA/CP-199902085526/SUPPL1)

51. D. Stauffer, A. Aharmony (1994) Introduction to Percolation Theory. $2^{\text {nd }}$ Ed., Tayler and Francis London

52. Y. Wang, J. Hu, Y. Lin, C-W. Nan (2010) Multiferric magnetoelectric composite nanostructure. NPG Asia Mate, 2(2):61-68

53. L.W. Martin, S.P. Crane, Y-H. Chu, M.B. Holcomb, M. Gejek, M. Huijben, C-H.Yang, N. Blake, R.Ramesh (2008) Mulitferrics and magnetoelectrics: thin films and nanostructures. J.Phys: Conden.Matter, 20:434220

54. T.Titscher, J.F.Unger (2015) Application of molecular dynamics for the generation of dense mesoscale geometries, BAM Federal Institute Materials Research and Testing. Computers and Structures, 158:274-284

55. A. Donev, S. Torquato, F.H. Stillinger, R. Connely (2003) Jamming in hard sphere and disk packings. J.Appl. Phys., 95(3):989-999

56. P.N. Pusey, E. Zaccarelli, C. Valeriani, E. Sanz, W.C.K. Poon, M.E. Cates (2009) Hard spheres: crystallization and glass formation. Phil.Trans., Royal Society A, 367(1909):4993-5011 
57. W. Schaertl, H. Sillescu (1994) Browian dynamics of poly disperse colloidal hard spheres: equilibrium structures and random close packings. Journal of Statistical Physics, 77(5/6):10071025

58. P. Jalali, M. Li (2004) An estimate of random close packing density in monodisperse hard spheres. Journal of Chemical Physics, 120(2):1138

59. N. Bell, Y. Yu, P.J. Nucha (2005) Particle-based simulation of granular materials. Eurographics/ACM SIGGRAPH, Univ.of Illinois

60. M. Rintoul, S. Torquato (1996) Metastability and Crystallization in Hard-Sphere Systems. Physical Review Letters, 77(20):4198-4201

61. M.Rintoul, S.Torquato (1996) Computer simulation of dense hard-sphere systems. J.Chem.Phys. 105(20): 9258

62. D.C. Rapaport (2014) Molecular dynamics simulations: a tool for exploration and discovery using simple models. J. Phys: Condens. Matter, 26:503104

63. S.W. Meier, R.M. Lueptow, J.M. Ottino (2007) A dynamical systems approach to mixing and segregation of granular materials in tumblers. Adv. in Phys., 56(5):757-827

64. K. Zhang, W.W. Smith, M. Wang, Y. Liu, J. Schroers, M.D. Shattuck, C.S. OHern (2014) Connection between the packing efficiency of binary hard spheres and the glass-forming ability of bulk metallic glasses. Physical Review E, 90: 032311

65. C. Radin (2008) Random close packing of matter. J. Stat Phys, 131:567-573

66. Y. Ishii, K. Sato, M. Salanne, P.A. Madden, N. Ohtori (2016) Thermal conductivity of simple liquids: origins of temperature and packing fraction dependencies. Journal of Chemical Physics, $140: 114502$

67. E. Santiso, E.A. Muller (2002) Dense packing of binary and polydisperse hard spheres. Molecular Physics, 100(15):2461-2469

68. E.J. Garboczi, M.F. Thorp, M.S. DeVries, A.R. Ray (1991) Universal conductivity curve for plane containing random holes. Physical Review A, 43(12):6473-6482 
69. E.J. Garboczi, D.P. Bentz (Oct 1996) Multi-scale picture of concrete and its transport properties: introduction for non-cement researchers. U.S. National Institute of Standards and Technology, NISTIR 5900

70. Y.C.Chiew, E.D.Glandt (1983) Percolation behavior of permeable and of adhesive Spheres. J. Phys. A: Math. Gen., 16:2599-260

71. B. Lamas, B. Abreu, A. Fonseca, N. Martins, M. Oliveira (2013) Numerical analysis of percolation formulation in carbon nanotube based nanofluids. Int. J. Num. Meth. Eng. Vol.95:257-270

72. D.P. Benz, E.J. Garboczi (1991) Percolation of phases in a three-dimensional cement paste microstructural model. NIST, 21(2/3):325-344

73. H.Gould, J. Tobochnik (1996) An introduction to computer simulation methods. $2^{\text {nd }}$ Ed., Chap.13 Percolation, Addison Wesley

74. A. Noel, J. Faucheu, J-M. Chenal, J-P. Viricell (2014) Electrical and Mechanical Percolation in graphene-latex Nanocomposites. Polymer, 55:5140-5145

75. Y. Zhu, K. Chen, F. Kang (2013) Percolation transition in thermal conductivity of $\beta-\mathrm{Si}_{3} \mathrm{~N}_{4}$ filled with epoxy. Solid State Communications, 158:46-50

76. N.S.Sharif, J.J. Mohamed, H.S.Derita, Z.A.Ahmad, M.Z.Abdullah, H.Mohamad, W.A.W.Yusoff (2014) The effect of sintering conditions on the microstructure and electrical properties of $\mathrm{Pb}\left(\mathrm{Zr}_{0.15} \mathrm{Ti}_{0.48}\right) \mathrm{O}_{2}$ Ceramic. Journal of Mechanical Engineering and Sciences, 6:901-906 


\title{
Chapter 2
}

\section{Electrical percolation threshold of magnetostrictive inclusions in a piezoelectric matrix composite as a function of relative particle size}

\begin{abstract}
Magnetoelectric (ME) composites can be produced by placing magnetostrictive particles in a piezoelectric-matrix. Ferrite magnetostrictive particles, if allowed to percolate, can short the potential difference generated in the piezoelectric phase. Modeling an ME composite as bi-disperse hard shells, with the magnetostrictive $(\mathbf{H})$ particles scaled to $100 \mathrm{~nm}$, molecular dynamics was used to explore relationships among relative particle size, particle affinity, and electrical percolation with the goal of maximizing the percolation threshold. Two factors raise the percolation threshold, namely the size of magnetostrictive particles relative to the piezoelectric $(\mathbf{E})$ particles, and the affinity between the magnetostrictive and piezoelectric particles.
\end{abstract}

Key words: magnetostrictive, piezoelectric, magnetoelectric composite, percolation, granular molecular dynamics, LAMMPS, segregation, polydisperse hard shells, Leonard-Jones

1. Introduction and objectives

Magnetostriction is a property of ferromagnetic materials that causes them to deform with strain when exposed to a magnetic field [1-8]. For example, Cobalt ferrite $\mathrm{CoFe}_{2} \mathrm{O}_{4}(\mathrm{CFO})$ is a ceramic ferrite with high magnetostrictive coupling [5]. The piezoelectric effect [4] [7] is the ability to generate electrical potential in response to an applied mechanical strain [9]. For example, pervoskite $\mathrm{Pb}\left[\mathrm{Zr}_{0.52} \mathrm{Ti}_{0.48}\right] \mathrm{O}_{3}$ (PZT) is a chemically stable and hard material with high piezoelectric coupling [10]. Magnetoelectric (ME) composites combine magnetostrictive and piezoelectric materials into a composite material that can convert a magnetic field into an electrical potential [1-8]. 
Since magnetostrictive (H) materials are electrically conductive [1-3], once the $\mathbf{H}$ particles percolate, the charge produced by the piezoelectric $(\mathbf{E})$ materials is lost, so a high percolation threshold is needed to achieve high magnetoelectric performance [11-16]. Therefore, the objective of this study is to find the largest volume fraction or percolation threshold for which electrical percolation of the $\mathbf{H}$ phase does not occur, as a function of the relative particle size and affinity between the precursor powders.

The ratio of the volume of $\mathbf{H}$ particles to the volume of the simulation box is denoted by $\boldsymbol{\rho}=\mathrm{V}_{\mathrm{H}} / \mathrm{box}$ volume. A mixture of two powders, one conductive and the other insulating, represented by spheres of equal diameter has a percolation threshold of $\boldsymbol{\rho}_{\mathbf{c}}=15.4 \%$ regardless of the arrangement of the spheres into any type of lattice [11] [17]. The volume ratio $\boldsymbol{\rho}$ may be written as $\boldsymbol{\rho}=\mathbf{f} \times \mathbf{p}$ where $\mathbf{f}$ is the packing fraction of both powders in the container, and $\mathbf{p}=\mathrm{V}_{\mathrm{H}} /\left(\mathrm{V}_{\mathrm{H}}+\mathrm{V}_{\mathrm{E}}\right)$ is the volume fraction [17]. Interstitial space is always present between packed hardshell spheres of any size. If the interstitial space between the $\mathbf{H}$ spheres is somehow filled completely then $\mathbf{f}=1$, and volume ratio $\boldsymbol{\rho}$ and volume fraction $\mathbf{p}$ are numerically equal. However, if the interstitial space between $\mathbf{H}$ particles is reduced by sintering, then the volume fraction (p) and the volume ratio $(\boldsymbol{\rho})$ increases since $\mathbf{p}_{\mathbf{c}}=\boldsymbol{\rho}_{\mathbf{c}} / \mathbf{f}$ where $\mathbf{f}$ is measured before sintering.

Numerous researchers have studied the effects of particle size on rheology and flow patterns [18][19] and granular segregation [20][21], and numerous researchers have applied molecular or particle dynamics to the study of granular motion [18-21]. Kuzy [23] studied the effects of particle size on granular static spatial distribution patterns and, in particular, studied the percolation threshold $\left(\boldsymbol{\rho}_{\mathbf{c}}\right)$ when $\boldsymbol{\rho}_{\mathbf{c}}=\mathbf{p}_{\mathbf{c}}$ for a fine-grain conductive phase $\mathbf{H}$ with spherical particle radius $\mathbf{R}_{\mathbf{H}}$ dispersed and surrounding larger spherical particles of phase $\mathbf{E}$ with radius $\mathbf{R}_{\mathbf{E}}$. He concluded (Fig. 1 lower right insert) that the larger the $\mathbf{R}_{\mathrm{E} / \mathbf{R}_{\mathbf{H}}}$ ratio, the lower the percolation threshold of the $\mathbf{H}$ phase. Our study is the diametric opposite of Kuzy's; that is, to increase the percolation threshold of the larger $\mathbf{H}$ phase (Fig. 1 upper left insert) by reducing the size of particles in the $\mathbf{E}$ phase. One goal of this study is to test via molecular dynamics 
simulation the proposition that the percolation threshold of the $\mathbf{H}$ phase will increase as the size of the particles in the $\mathbf{E}$ phase decreases [11-12] [20][23][24].

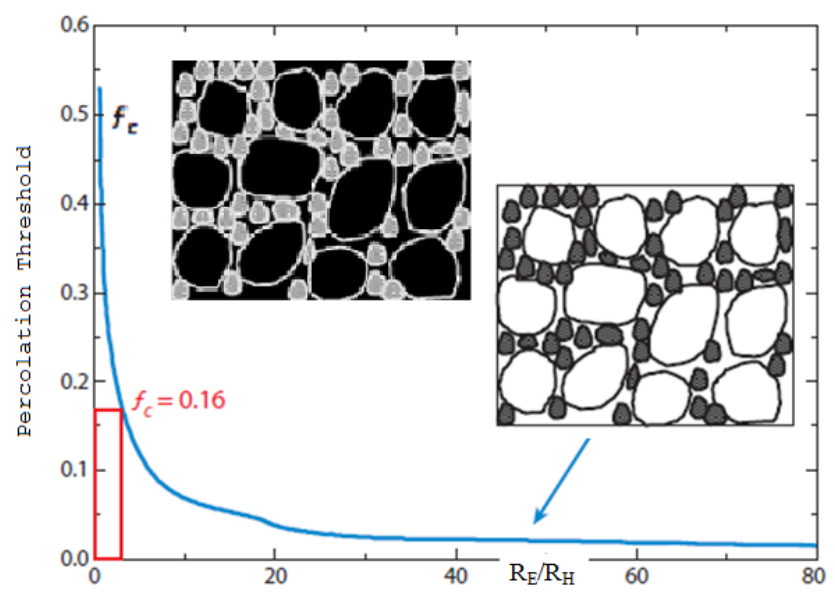

Fig. 1 [11] Percolation threshold as a function of relative particle size $R_{E} / R_{H}$. Insert lower right: smaller $\mathrm{H}$ dark particles; upper left: larger $\mathrm{H}$ dark particles

\section{Methodology}

\subsection{Particle Dynamics}

In this study, particle dynamics is preformed using Large Scale Atomic and Molecular Massively Parallel Simulation (LAMMPS)@ software from Sandia National Laboratories [34] to simulate the mixing of two powders that represent the magnetostrictive $\mathbf{H}$ and piezoelectric $\mathbf{E}$ phases. Particles are modeled as perfect spheres of uniform density for each phase, but each phase has a different diameter with a ratio between the two diameters given by $\mathbf{R}_{\mathbf{H}} / \mathbf{R}_{\mathrm{E}}$. The Leonard-Jones (L-J) “12-6” potential is used in this study to model particles interactions where distance is expressed in units of sigma $(\boldsymbol{\sigma})$ and energy in units of epsilon ( $\boldsymbol{\varepsilon}$ ) which are features of the pair-wise L-J potential function between particles centers (Fig. 2). The diameter $\mathbf{D}$ the CFO particles were chosen to be $100 \mathrm{~nm}(\mathrm{D}=1.12246 \sigma)$ as a compromise between the effects of quantum tunneling [25-31] and mechanical coupling [1-2] [13-16]. If the particles are too large, mechanical coupling between the $\mathbf{E}$ and $\mathbf{H}$ phase will be poor. If the particles are too small, 
quantum tunneling will short any voltage produced in the $\mathbf{E}$ phase. The L-J potential can be used to model hard shell spheres with the particle diameter modeled as the cutoff corresponding to the deepest point in the well which is located at $1.12246 \sigma$ (Fig. 2). The Leonard-Jones potential is set equal to zero after after the range cutoff $\mathbf{R}_{\mathbf{m}}$ (Sect. 2.4).

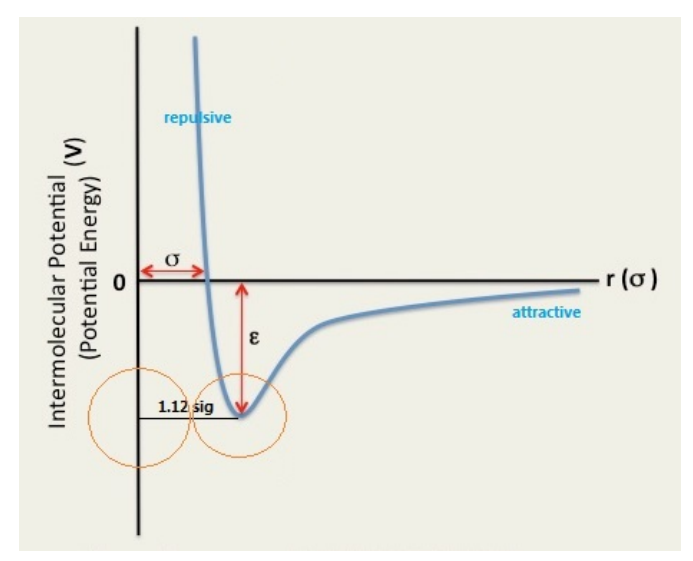

Fig. 2 Leonard-Jones pair-wise potential energy

To approximate the hard-shell behavior, the well depth (Fig. 2) was set to $10 \varepsilon$ during the mixing stage and $20 \varepsilon$ during the equilibration and gelation stage. Per preliminary studies using Hertzian analysis [32][33] to compare Leonard-Jones well depth to particle elasticity, a well depth of $10 \varepsilon$ to $20 \varepsilon$ produces a stress which is at least three orders of magnitude larger than any stress which would be produced by gravity on either the $\mathbf{H}$ or $\mathbf{E}$ particles. Therefore, the effects of gravity may be ignored. To simulate particle friction and affinity, an attractive force is added between the $\mathbf{H}$ and $\mathbf{E}$ particles while no other forces are present. This was accomplished by extending the L-J cutoff between the $\mathbf{H}$ and $\mathbf{E}$ particles from $\mathbf{r}_{\mathbf{c o}}=1.12246 \sigma$ to $\mathbf{r}_{\mathbf{c o}}=1.25 \sigma$. The value $1.25 \sigma$ was chosen because too large a cutoff violates the rigid spheres assumption that particles do not attract each other. If particles attract each other over long distances, then the distance between the particles decreases, thus enhancing the likelihood of $\mathbf{H}$ particle percolation contrary to our goals.

\subsection{Simulation Process}


We require $\mathbf{H}$ and $\mathbf{E}$ particles to be well mixed with each other to maintain high electromechanical energy transfer between them. All simulations are time integrated with the Verlet velocity algorithm [34] using the Nose-Hoover NVT thermostat where the number and volume are fixed and temperature is either fixed or slowly decreased. To prevent segregation, a common problem when mixing powders [23][3540], the simulations are performed in three separate stages of mixing, equilibration, and gelation.

In the first stage (mixing) all particles are initially mixed at an L-J temperature of $T=0.2 \varepsilon / \mathrm{k}_{\mathrm{B}}\left(\mathrm{k}_{\mathrm{B}}\right.$ is the Boltzman constant which equals one in Leonard-Jones units) for 10 million time steps for $R_{H} / R_{E}=1$, 2, and 5 million time steps for $\mathrm{R}_{\mathrm{H}} / \mathrm{R}_{\mathrm{E}}=3$. In all cases, periodic boundary conditions are applied. For equally sized spheres the maximum packing fraction, below which the particles are free to move, is $\mathbf{f}=0.64$ [39]. For any packing fraction above this value, the particles will experience a glass transition [36] [39] and thus are not be able to move or mix. Therefore, for the mixing stage, the packing fraction used was $\mathbf{f}=0.5236$ corresponding to the simple cubic (sc) lattice. This is sufficiently less than $\mathbf{f}=0.64$ to allow mixing to occur.

The second stage (equilibration) is where local particle movement on the order of particles size is allowed, but motion on the order of the size of the simulation box is restricted. The equilibration stage is run for 100,000 time steps with the L-J parameters $\boldsymbol{\sigma}$ and the cutoff $\mathbf{r}_{\mathbf{c o}}$ adjusted to increase the particle size, so that the particles occupy more space for a fixed simulation box volume, effectively increasing the packing fraction from $\mathbf{f}=0.5236$ to $\mathbf{f}=0.553$. During the second stage (equilibration) the simulation is run at a cold temperature ramped from $\mathrm{T}=0.02 \varepsilon / \mathrm{k}_{\mathrm{B}}$ to $0.01 \varepsilon / \mathrm{k}_{\mathrm{B}}$ allowing sufficient energy for the particles to move locally to find their equilibrium positions but restrict particle movement sufficiently to avoid particle segregation [23] [35-40]. Finally, the third stage (gelation) is run for 10,000 additional time steps where the temperature is also ramped from $T=0.02 \varepsilon / \mathrm{K}_{\mathrm{B}}$ to $0.01 \varepsilon / \mathrm{k}_{\mathrm{B}}$, to allow sufficient energy for motion while the L-J interaction between the particles and the box walls is adjusted to effectively decrease the box size to achieve the target packing fraction of $\mathbf{f}=0.64$ for $R_{H} / R_{E}=1$ or $\mathbf{f}=0.675,0.73$ for $\mathrm{R}_{\mathrm{H}} / \mathrm{R}_{\mathrm{E}}=2,3$ respectively. 


\subsubsection{Packing Fraction}

To achieve a packing fraction of $\mathbf{f}=0.64,0.675$, or 0.73 without segregation, particles of diameter $1.12246 \sigma$ are mixed in a simulation box of side length equal to $20 \times 1.12246 \sigma$ at a packing fraction of 0.5236 with periodic boundary conditions applied. In the equilibration stage to apply isotropic compression [20] for the case of $R_{H} / R_{E}=1$, the particles radii are grown by a factor of $(0.64 / 0.5236)^{1 / 3}$, and for $\mathrm{R}_{\mathrm{H}} / \mathrm{R}_{\mathrm{E}}=2$, and 3, the particle diameters are increased by a factor of $(0.675 / 0.5235)^{1 / 3}$, and $(0.73 / 0.5235)^{1 / 3}$ respectively. For $R_{H} / R_{E}=1$ this scaling factor would increase the packing fraction to $0.5236 \times(0.64 / 0.5236)=0.64$, but by design with reflective boundary conditions, the particle centers can go all the way to the edge of the box in the equilibration stage which decreases the packing fraction to $0.64 \times(20 / 21)^{3}=0.552856$. This packing fraction is still sufficiently below $\mathbf{f}=0.64$ (or $0.675,0.73$ ) to allow local particle movement, but is sufficiently high to prevent phase segregation, as evidenced by the measurements for the center of mass $(\mathrm{CoM})$, average local volume fraction $\left(\mathbf{p}_{\mathbf{a}}\right)$, probability density profile (PDP), and radial distribution function (RDF) (see section 2.3). To restore the packing fraction back to 0.64 (or $0.675,0.73$ ) the simulation box sides are shrunk in the gelation stage by a factor of (20/21), thus removing the reduction in packing fraction $\mathbf{f}$ that resulted from using periodic boundary conditions.

\subsubsection{Particle Size}

Simulations were conducted for three relative particle sizes of $R_{H} / R_{E}=1,2$, 3 where $R_{H}$ and $R_{E}$ are the radius of the conductive $\mathbf{H}$ and insulating $\mathbf{E}$ phase particles, respectively. $R_{H}$ is fixed at $100 \mathrm{~nm}$, and the density of the particles is scaled to correspond to CFO and PZT molecular weights. When the $\mathbf{H}$ and $\mathbf{E}$ particles are of equal size, the simulation box side length is $1.12246 \sigma$ times the cube root of the number of particles being simulated. Specifically, for $20^{3}$ total spheres the length of the simulation box is $1.12246 \sigma \times 20=22.4492 \sigma$. Since the size of the box and the number of $\mathbf{H}$ particles remains unchanged throughout this study, to maintain the volume fraction (p) and the packing fraction (f) unchanged, the 
number of $\mathbf{E}$ particles is varied with $\left(\mathrm{R}_{\mathrm{H}} / \mathrm{R}_{\mathrm{E}}\right)^{3}$. In the gelation stage, the simulation uses rigid walls which are constructed with Leonard-Jones particle-wall interactions equal to the particle-particle interactions except where in a few cases when $\mathrm{R}_{\mathrm{H}} / \mathrm{R}_{\mathrm{E}}=3$, due to computational difficulties, it became necessary to reduce the strength of the particle-wall interaction a factor of 4 to 10, but analysis of the results suggests that the percolation threshold was not affected.

\subsection{Characterization}

Measurements for this study include the radial distribution function (RDF), probability density profiles (PDP), center of mass (CoM), and average local volume fraction ( $\left.\mathbf{p}_{\mathbf{a}}\right)$ which were used to characterize the degree of mixing.

\subsubsection{Radial Distribution Function}

The radial distribution function (RDF) maps the distances among pairs of particles $\mathbf{H}-\mathbf{H}, \mathbf{E}-\mathbf{E}$, and $\mathbf{H}$ $\mathbf{E}$ to the likelihood of finding pairs of particles at those distances from each other. These functions characterize the structure between phases as well as mean separation distances which are a key parameter for understanding percolation [12][25][41][42][55].

\subsubsection{Probability Density Profiles}

Probability density profiles (PDP) are histograms of the number of particles (both $\mathbf{H}$ and $\mathbf{E}$ ) which in this study are computed between $y$ and $y+\Delta y$ along the $y$ axis of the simulation box, where $\Delta y$ represents the histogram bin size which is taken as one eight the length of the simulation box. The PDP gives a measure of the degree of mixing between $\mathbf{H}$ and $\mathbf{E}$ particles. The percent difference is defined as the difference between the maximum and minimum values of density divided by the average density. Percent differences less than $10 \%$ typically indicate good mixing. On the other hand if the particles segregate, a sharp inflection or abrupt value change in the PDP is observed.

\subsubsection{Center of Mass}


The center of mass (CoM) is computed as a function of time in L-J time steps along a single direction of the simulation box for the $\mathrm{H}$ particles only. A well mixed distribution of $\mathbf{H}$ particles will have a CoM within plus or minus $5 \%$ of the center of the box after the simulation is completed. A CoM outside this 5\% range provides an indication that segregation has occurred.

\subsubsection{Average Local Volume Fraction}

The average local volume fraction $\left(\mathbf{p}_{\mathbf{a}}\right)$ is an intrinsic scale invariant quantity proportionate to the volume fraction measured repeatedly within small spheres of radius twice the radius of an $\mathbf{H}$ particle for all particles both $\mathbf{H}$ and $\mathbf{E}$ for any given simulation. For example, for a simulation volume fraction of 0.18, if $12 \mathbf{H}$ particles of diameter $\mathbf{D}$ were found in a sphere of diameter $2 \mathbf{D}$ centered around any given particle, and $64 \mathbf{E}$ particles were found in that same sphere, then $\mathbf{p}_{\mathbf{a}}$ for that point would be $12 / 64 \times 100=$ 0.1875 . This calculation is repeated for all particle centers and averaged. In order to compare $\mathbf{p}_{\mathbf{a}}$ values for simulations of unequal number of $\mathbf{E}$ particles, the number of $\mathbf{E}$ particles is normalized by dividing the number of $\mathbf{E}$ particles found in any $2 \mathbf{D}$ sphere by $\left(\mathrm{R}_{\mathrm{H}} / \mathrm{R}_{\mathrm{E}}\right)^{3}$.

\subsection{Percolation Distance}

The percolation distance $\mathbf{R m}$ or critical range between particle centers for which percolation first occurs is the smallest distance between adjacent particle centers for which pairs of connected particles are chained together to produce a percolation path from one side of the simulation box to the other. Any distance larger than $\mathbf{R}_{\mathbf{m}}$ will also produce a percolation path, but the critical percolation distance is always the smallest distance among coordinate centers which produces a percolation. The percolation distance $\mathbf{R}_{\mathbf{m}}$ includes the effective particle diameter after growth (section 2.4.1) and the effect from quantum tunneling (section 2.4.2). Because the growth or scaling factor is different for different packing fractions at which glass transition is expected, $\mathbf{R}_{\mathbf{m}}$ is calculated as follows:

$$
\begin{aligned}
& \mathbf{R}_{\mathbf{m}}=\text { particle diameter } \times \text { scale factor } \times \text { quantum tunneling } \\
& \mathrm{R}_{\mathrm{H}} / \mathrm{R}_{\mathrm{E}}=1 \rightarrow \mathrm{R}_{\mathrm{m}}=1.12246 \sigma \times(0.64 / 0.5236)^{1 / 3} \times 1.05=1.26 \sigma
\end{aligned}
$$




$$
\begin{aligned}
& \mathrm{R}_{\mathrm{H}} / \mathrm{R}_{\mathrm{E}}=2 \rightarrow \mathrm{R}_{\mathrm{m}}=1.12246 \sigma \times(0.675 / 0.5236)^{1 / 3} \times 1.05=1.28 \sigma \\
& \mathrm{R}_{\mathrm{H}} / \mathrm{R}_{\mathrm{E}}=3 \rightarrow \mathrm{R}_{\mathrm{m}}=1.12246 \sigma \times(0.73 / 0.5236)^{1 / 3} \times 1.05=1.32 \sigma
\end{aligned}
$$

\subsubsection{Particle scaling factor (SF)}

The maximum packing fraction of equally sized random close packed spheres that can be achieved without particle deformation is $\mathbf{f}=0.64$ for $R_{H} / R_{E}=1, \mathbf{f}=0.675$ for $R_{H} / R_{E}=2$, and $\mathbf{f}=0.73$ for $R_{H} / R_{E}=3$ [38][39]. When powders or hard shell spheres are mixed, segregation between non-identical particles can occur when the particles are under stress or pressure [23] [35-40]. In order to achieve a packing fraction of $\mathbf{f}=0.64$ while avoiding phase segregation, in the case of $R_{H} / R_{E}=1$, the particle diameters are increased in the equilibration stage by a scaling factor of $(0.64 / 0.5235)^{1 / 3}=1.069203$. This increases the diameter of the particles from $1.12246 \sigma$ to $1.200137 \sigma$. In the case of $\mathrm{R}_{\mathrm{H}} / \mathrm{R}_{\mathrm{E}}=2$, and 3 , the particle diameters are increased by a factor of $(0.675 / 0.5235)^{1 / 3}$, and $(0.73 / 0.5235)^{1 / 3}$ respectively (section 2.2 .1$)$.

\subsubsection{Quantum Tunneling (QT)}

Quantum tunneling [43] is an effect whereby electric charge can jump a space between conductive but electrically isolated particles that is forbidden by classical mechanics. Quantum tunneling is reported to range from $3 \mathrm{~nm}$ [25] to $10 \mathrm{~nm}$ [31] between conducting particles in a non-conducting matrix. Others [28][29] describe plasmonic (charge density) energy transfer partially attributed to tunneling between conducting particles separated by distances up to $7 \mathrm{~nm}$. Hill et al. [27] reports plasmonic conduction between particles exceeding a separation distances of $20 \mathrm{~nm}$. Therefore we decided that if two conducting particles were at most $5 \mathrm{~nm}$ or closer, there would be a high likelihood of quantum tunneling between the two conducting particles of diameter $100 \mathrm{~nm}$, so when the $\mathrm{H}$ particle diameter $\mathbf{D}=$ $100 \mathrm{~nm}$, the conductivity distance used to evaluate percolation between particles centers is increased by a factor of 1.05 .

\subsection{Percolation Algorithm}


The algorithm to measure percolation consists of two subroutines. The first subroutine dist3.c (Appendix A) builds a pair bonding list of all pairs of $\mathbf{H}$ particles that are less than $\mathbf{R}_{\mathbf{m}}$ from each other. The second subroutine zap2.c (Appendix A) attempts to find a path from one side of the box (coordinate less than 10\%) to the other side (coordinate greater than 90\%), refered to below as endzone locations, by chaining together successive pairs of particles via recursion [44].

Let $\mathbf{R}$ represent a variable distance. To compute the percolation threshold $\left(\boldsymbol{\rho}_{\mathbf{c}}\right)$, the theoretical distance between $\mathrm{H}$ particles at which a percolation could occur $\left(\mathbf{R}_{\mathbf{m}}\right)$ is determined for a given volume fraction $\mathbf{p}$ and packing fraction $\mathbf{f}$. Next, the distance algorythm dist3.c followed by the recursive percolaton algorithm zap2.c (Appendix A) is run to test for percolation for a range of $\mathbf{R}$ values below and above $\mathbf{R}_{\mathbf{m}}$. If a percolation is discovered for $\mathbf{R}<=\mathbf{R}_{\mathbf{m}}$, then the $\mathbf{M E}$ composite is judged to have percolated, and the distance $\mathbf{R}$, at which the percolation occurred, is also used to compute the corresponding c.n. for that percolation threshold. If a percolation does not occure until $\mathbf{R}>\mathbf{R}_{\mathbf{m}}$, then the ME composite is judged not to have percolated, but the value of $\mathbf{R}$, is still increased and the percolation algorithm is repeated until a percolation is found. Eventually for large enough $\mathbf{R}$, all configurations of $\mathbf{H}$ particles will percolate. Regardless of whether the ME composite is judged to have percolated or not, the smallest distance at which a computational percolation occurred is used to compute the $\mathbf{H}-\mathbf{H}$ coordination number (c.n.). The c.n. is used to show that the average number of connections of each particle to nearby particles is approximately independent of the volume fraction $\mathbf{p}$. The results are shown in Table 2.

A distance along the simulation box's side length and within $10 \%$ of the box perpendicular edge was chosen as an endzone distance because the particles centers cannot get closer than $0.627 \sigma$ from either simulation wall adjusting for particle growth in the equilibration stage. This causes a dearth of particle centers in the first and last slice closest to the walls as shown in Fig. 5 (section 3.2). Since the box length is $22.4492 \sigma, 10 \%$ of this is rounded to $2.25 \sigma$. The second to last slice where a full population of particles is expected to occur is between $0.63 \sigma$ and $1.9 \sigma$. Adding a small safety factor of $0.35 \sigma$ to account for 
steric effects, if any percolation path gets within $2.25 \sigma$ or $10 \%$ of the box length to a wall, a conductive path is presumed.

\subsection{Affinity}

As all the particles are compressed, for the cases when $\mathbf{R}_{\mathbf{H}} / \mathbf{R}_{\mathbf{E}}$ is not equal to one, the smaller $\mathbf{E}$ particles are likely to move to the interstitial space between the large $\mathbf{H}$ particles rather than maintain positions that separate $\mathbf{H}$ particles from each other. Friction between the $\mathbf{H}$ and $\mathbf{E}$ particles would reduce this tendency, but the Leonard-Jones potential used in this study produces a purely radial force between particles without a shear or frictional component. The effect of friction may be partially simulated, however, by a radial attractive force between the $\mathbf{H}$ and $\mathbf{E}$ particles. A radial force between the $\mathbf{H}$ and $\mathbf{E}$ particles is implemented by extending the L-J cutoff parameter $\mathbf{r}_{\mathbf{c o}}$ between the $\mathbf{H}$ and $\mathbf{E}$ particles. In our study, we extended the L-J from $\mathbf{r}_{\mathbf{c o}}=1.12246 \sigma$ to $\mathbf{r}_{\mathbf{c o}}=1.25 \sigma$. The value $1.25 \sigma$ was chosen to allow the $\mathbf{H}$ particles to attract the $\mathbf{E}$ particles close to the surface of the $\mathbf{H}$ particles, but to be short range enough so as not to allow long range forces to do work on the system.

In addition, even in the absence of friction, a slight attractive force between $\mathbf{H}$ and $\mathbf{E}$ particles allows the smaller $\mathbf{E}$ particles to act like a coat of paint surrounding and insulating the conductive $\mathbf{H}$ particles which would increase the percolation threshold. Functionalization of CFO particles into polymers has been considered by [45] whereby similar techniques might be used to produce affinity between $\mathbf{H}$ and $\mathbf{E}$ particles.

\section{Results}

The critical percolation threshold $\boldsymbol{\rho}_{\mathbf{c}}$ of phase $\mathbf{H}$ is expressed as $\rho_{\mathrm{c}}=\mathrm{V}_{\mathrm{H}} / \mathrm{V}_{\mathrm{box}}=\mathrm{f} \times \mathrm{p}_{\mathrm{c}}$ where $\mathbf{f}$ is the packing fraction, $\mathbf{V}_{\mathbf{H}}$ is the volume of the $\mathrm{H}$ particles, $\mathbf{V}_{\mathbf{b o x}}$ is the simulation box volume, and $\mathbf{p}_{\mathbf{c}}$ is the critical volume fraction where volume fraction $\mathrm{p}=\mathrm{V}_{\mathrm{H}} /\left(\mathrm{V}_{\mathrm{H}}+\mathrm{V}_{\mathrm{E}}\right)$. The critical percolation threshold $\boldsymbol{\rho}_{\mathbf{c}}$ for equally sized hard-shell spheres without affinity is 0.154 [11] [17]. To verify our model, three different lattices, simple cubic (sc) (f=0.5236), body centered cubic (bcc) (f=0.6802), and hexagonal close packed 
(hcp) (f=0.7405) were evaluated for percolation. For all crystal lattices $\left(R_{H} / R_{E}=1\right.$ only), different volume fractions are achieved by randomly removing a precise number of phase $\mathbf{H}$ spheres at lattice points while leaving the remaining $\mathbf{H}$ spheres in place. The results are shown in Table 1 and are comparable to Table I of [17]. Referring to Table 1, c.n. stands for the lattice coordination number, $\mathbf{V}$ means volume, and $\mathbf{N}$ stands for number of particles for equations (2) (3).

For $\mathrm{R}_{\mathrm{H}} / \mathrm{R}_{\mathrm{E}}=1$, volume fraction is

$$
\mathrm{p}=\mathrm{N}_{\mathrm{PM}} /\left(\mathrm{N}_{\mathrm{PM}}+\mathrm{N}_{\mathrm{PE}}\right)=\mathrm{V}_{\mathrm{H}} /\left(\mathrm{V}_{\mathrm{H}}+\mathrm{V}_{\mathrm{E}}\right)
$$

In general, the volume ratio is

$$
\rho=f \times p
$$

The percolation threshold shown in Tables 1-2 are the lowest values of $\mathbf{p}$ and $\boldsymbol{\rho}$ for which percolation occurs, and are denoted $\mathbf{p}_{\mathbf{c}}$ and $\boldsymbol{\rho}_{\mathbf{c}}$.

\section{Table 1.}

(Percolation Thresholds for hcp, bcc, sc lattices with $\mathrm{R}_{\mathrm{H}} / \mathrm{R}_{\mathrm{E}}=1$ )

\begin{tabular}{|c|c|c|c|c|c|}
\hline Crystal 3D & c.n. & $\mathbf{f}$ & $\mathbf{p}_{\mathbf{c}}$ & $\boldsymbol{\rho}_{\mathbf{c}}$ & $\boldsymbol{\rho}$ Scher-Zallen [17] \\
\hline hcp & 12 & 0.7405 & 0.175 & 0.130 & 0.144 \\
\hline bcc & 8 & 0.6802 & 0.24 & 0.163 & 0.163 \\
\hline sc & 6 & 0.5326 & 0.32 & 0.168 & 0.162 \\
\hline
\end{tabular}
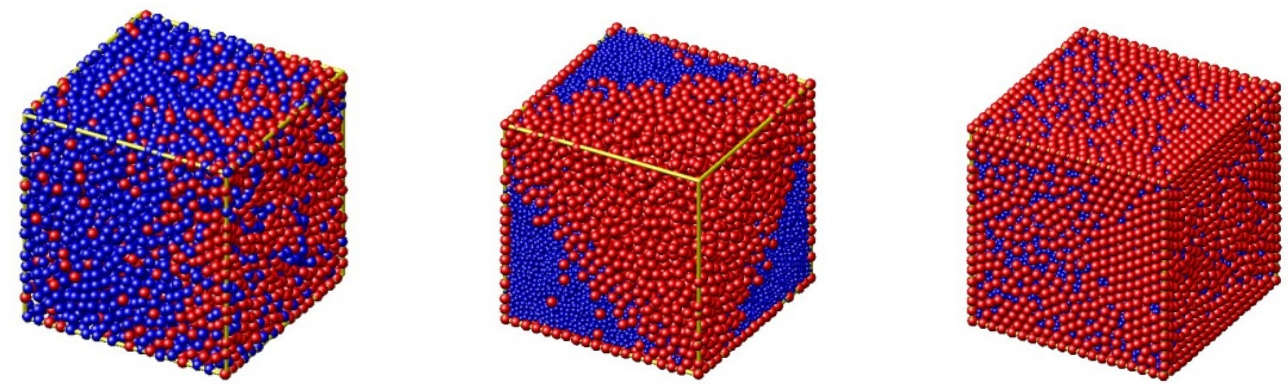

Fig. 3. Three different cases of segregation between red $\mathbf{H}$ and blue $\mathbf{E}$ particles

\subsection{Segregation}


When granular mixtures with different properties are subjected to stress or pressure, segregation as shown in Fig. 3 can occur [36] [46]. Segregation occurs between the different constituents [13-16] [23] [36] [47-50] due to difference in velocity, size, and density of the different particles groups. Segregation is detrimental to electromechanical coupling which requires the $\mathbf{H}$ and $\mathbf{E}$ particles to be thoroughly mixed. Percolation among one phase will either be undesirably enhanced within an aggregation of $\mathbf{H}$ particles which excludes $\mathbf{E}$ particles, or percolation will be inhibited if an agglomeration of $\mathbf{H}$ particles does not reach from one side to the other, but occupies just a central region. In this study, the glass transition [35] $[36][41][48][51][52]$ is used to restrict particle motion preventing segregation while the particles undergo equilibration and gelation. Glass transition is described as a sharp change from a viscous, rubbery, or fluid state to solid or gel state [35] [37] [52]. In this study we adopt the approach per [52] that at high packing fractions "arrest takes place via a glass transition process which can be driven by jamming as in hard sphere systems.”

\subsection{Random Close Packed Powders}

The closest that hard spheres can be packed into any lattice are with a coordination number of 12 and packing fraction of 0.7405 [53] which occurs for hcp and fcc lattices. If a lattice is relaxed and the spheres are allowed to move around randomly, the arrangement with the highest packing is called random close packing (rcp) with a packing fraction of 0.64 [17][22][37][38][40].

The results for simulations of random close packed powders using our methodology are shown in Table 2 and Fig. 4 with affinity (A) or no affinity (N) applied between $\mathbf{H}$ and $\mathbf{E}$ particles. The average local volume fraction $\mathbf{p}_{\mathbf{a}}$ described in section 2.14 is a measure of mixing. When mixing between the $\mathbf{H}$ and $\mathbf{E}$ phases is well maintained the average local volume fraction $\mathbf{p}_{\mathbf{a}}$ approaches $\mathbf{p}$. The average coordination number of the $\mathbf{H}$ particles computed at the smallest inter-particle distance between $\mathbf{H}$ particle centers that produces a percolation is denoted by c.n. $\mathbf{p}_{\mathbf{c}}=\mathrm{V}_{\mathrm{H}} /\left(\mathrm{V}_{\mathrm{H}}+\mathrm{V}_{\mathrm{E}}\right)$ is the threshold volume fraction 
of $\mathbf{H}$ phase with respect to the total particle volume. $\boldsymbol{\rho}_{\mathbf{c}}=\mathrm{V}_{\mathrm{H}}$ /box is the volume ratio of $\mathbf{H}$ to the simulation box.

Table 2. (mean values for 3 runs (COV[\%]),

$\left.\mathbf{R}_{\mathbf{m}}=1.26 \sigma, 1.28 \sigma, 1.32 \sigma, \mathbf{f}=0.64,0.675,0.73, \mathrm{D}=100 \mathrm{~nm}\right)$

\begin{tabular}{|c|c|c|c|c|c|}
\hline Affinity & $\mathbf{R}_{\mathrm{H}} / \mathbf{R}_{\mathrm{E}}$ & $p_{a}$ & c.n. & $\mathbf{p}_{\mathrm{c}}$ & $\rho_{c}$ \\
\hline $\mathbf{N}$ & 1 & $0.2429(10.19)$ & $1.69(16.22)$ & $0.2433(8.555)$ & $0.1557(8.384)$ \\
\hline $\mathbf{N}$ & 2 & $0.2780(4.893)$ & $2.02(7.967)$ & $0.2833(5.391)$ & $0.1913(5.589)$ \\
\hline $\mathbf{N}$ & 3 & $0.3213(3.962)$ & $1.897(3.390)$ & $0.320(5.413)$ & $0.2337(5.436)$ \\
\hline A & 1 & $0.2996(2.165)$ & $1.563(9.320)$ & $0.2967(1.946)$ & $0.190(1.823)$ \\
\hline A & 2 & $0.3375(2.923)$ & $1.7467(4.936)$ & $0.350(2.857)$ & $0.2363(2.753)$ \\
\hline A & 3 & $0.3984(6.305)$ & $1.55(0.0)$ & $0.3933(5.292)$ & $0.287(5.273)$ \\
\hline
\end{tabular}

Percolation vs. Relative Particles Size

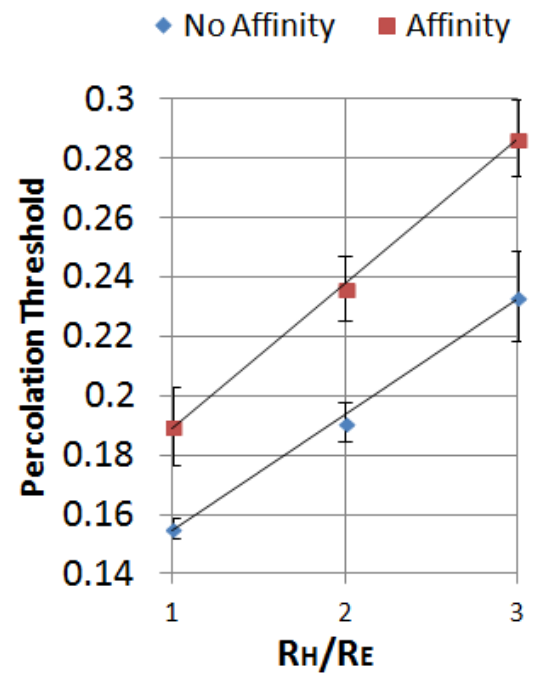

Fig. 4. Plot of percolation threshold ( $\left.\boldsymbol{\rho}_{\mathrm{c}}\right)$ versus particle size $\left(\mathrm{R}_{\mathrm{H}} / \mathrm{R}_{\mathrm{E}}\right)$ from Table 2

It can be seen that for $\mathrm{R}_{\mathrm{H}} / \mathrm{R}_{\mathrm{E}}=1, \boldsymbol{\rho}_{\mathrm{c}}$ reproduced the value of $0.1557(\sim 0.16)$ from the literature [11] [17]. For $R_{H} / R_{E}>1$, $\rho_{c}$ grows with $R_{H} / R_{E}(0.18,0.20)$, and more so with affinity $(0.22,0.27)$. With only three simulations per case, the standard deviation and coefficient of variance is reasonably small. The average coordination numbers (c.n.) are approximately invariant of the affinity and $R_{H} / R_{E}$ particle diameter ratio, and are less than an integer away from the number two, since every particle in the conduction path needs a minimum of two contact points to be part of a conductive chain. The c.n. is 
computed at the smallest $R_{m}$ value were a percolation is first detected for any given volume fraction (p). The average local volume fraction $\left(\mathbf{p}_{\mathbf{a}}\right)$ correlates well with the critical volume fraction $\mathbf{p}_{\mathbf{c}}$ indicating the $\mathbf{H}$ and $\mathbf{E}$ particles are well mixed.

The probability density profile and center of mass plot shown in Fig. 5 and 6 below are conducted as tests and verification of particle mixing. Complete mixing is required to ensure maximum $\mathrm{H}$ particle separation in order to minimize electrical percolation. A typical probability density profile (section 2.3.2) during the gelation stage is shown in Fig. 5. All density profiles show a percent difference less than $8 \%$ (excluding the first and last slice due to edge effects) indicating that the phases remains mixed through all three stages without segregation. A typical plot of the center of mass (CoM) (described in section 2.3.3) for the $\mathbf{H}$ particles is shown in Fig. 6. The CoM remains close to the center of the simulation box within $5 \%$ for all simulations in all stages again indicating good mixing without segregation.

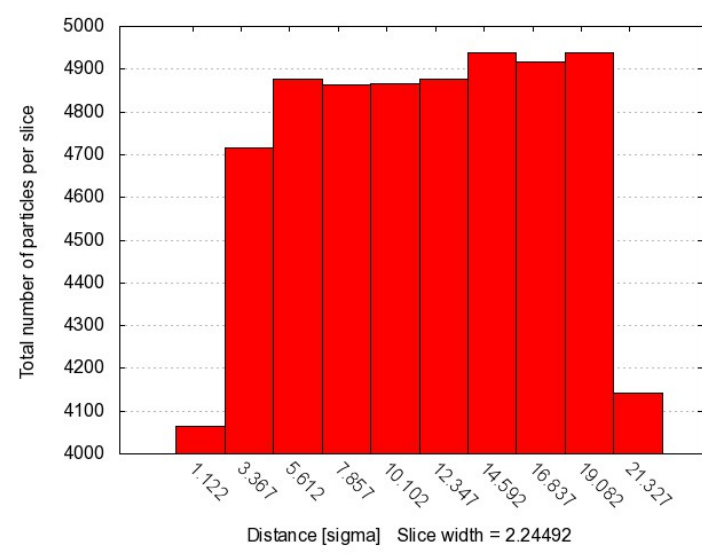

Fig. 5. Probability profile, gelation stage, $\mathrm{R}_{\mathrm{H}} / \mathrm{R}_{\mathrm{E}}=2$, Type:N, $\mathrm{p}=0.3$

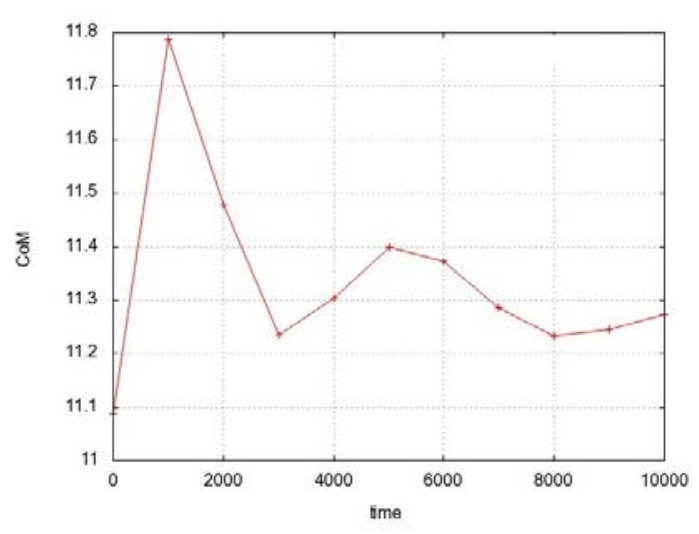

Fig. 6 . Center of Mass, gelation stage, $\mathrm{R}_{\mathrm{H}} / \mathrm{R}_{\mathrm{E}}=2$, Type: $\mathrm{N}, \mathrm{p}=0.3$

Renderings of particle positions after each stage of the simulation are shown in Figs. 7-14 with $\mathbf{H}$ particles in red and $\mathbf{E}$ particles in blue color. The radial distribution function RDF depicts morphological features and energy plots which attest to the equilibrium reached in each case. 


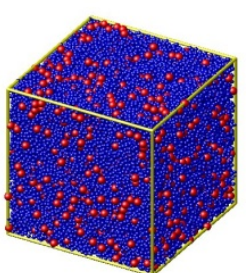

(a) PM:red PE:blue

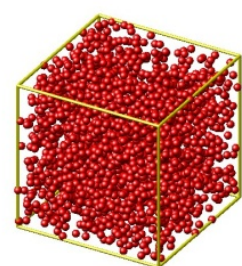

(b) PM only

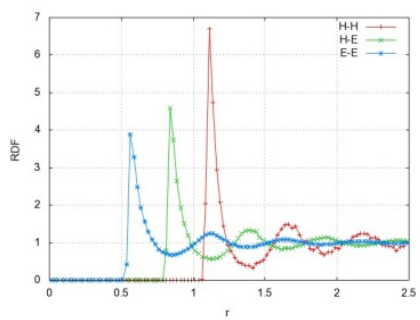

(c) $\mathrm{RDF}$

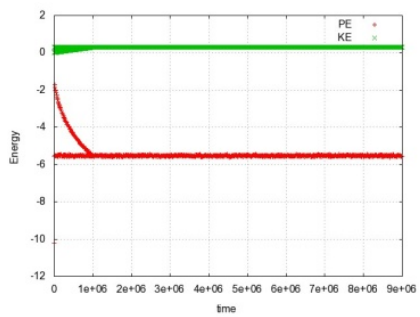

(d) Kinetic and Potential. Energy vs. time

Fig. 7 Mixing stage, $R_{H} / R_{E}=2 p=0.3$

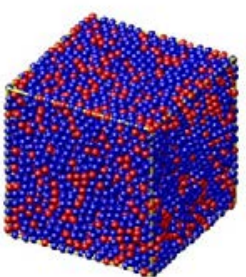

(a) PM:red PE:blue

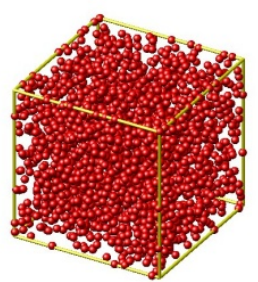

(b) PM only

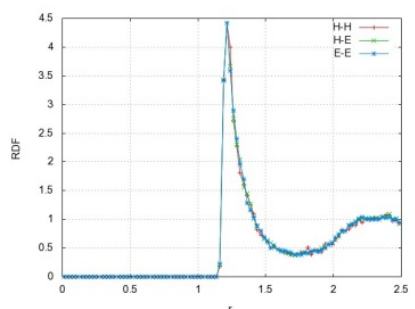

(c) $\mathrm{RDF}$

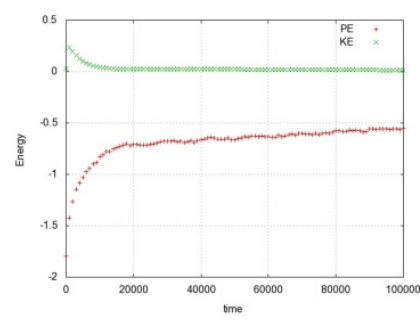

(d) Kinetic and Potential Energy vs. time

Fig. 8 Equilibration stage, $R_{H} / R_{E}=1$, Type: $N, p=0.3$

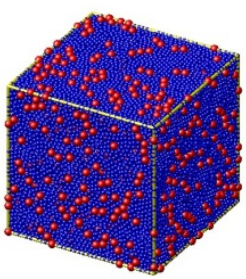

(a) PM:red PE:blue

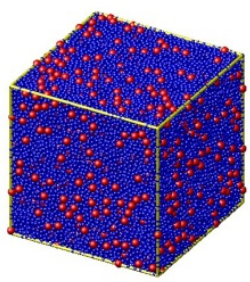

(a) PM:red PE:blue

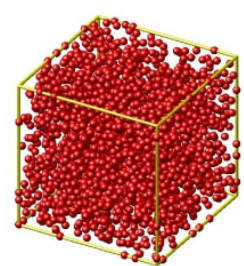

(b) PM only

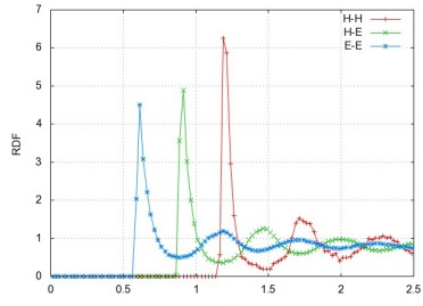

(c) $\mathrm{RDF}$

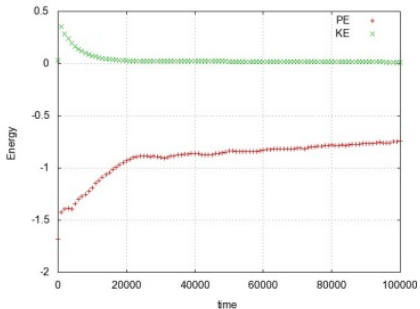

(d) Kinetic and Potential Energy vs. time

Fig. 9 Equilibration stage, $R_{H} / R_{E}=2$, Type: $N, p=0.3$

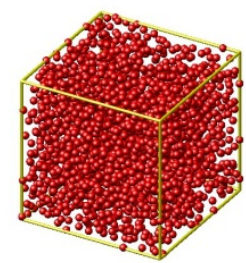

(b) PM only

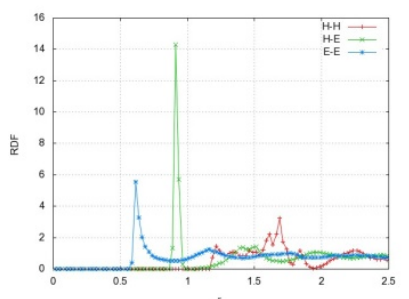

(c) $\mathrm{RDF}$

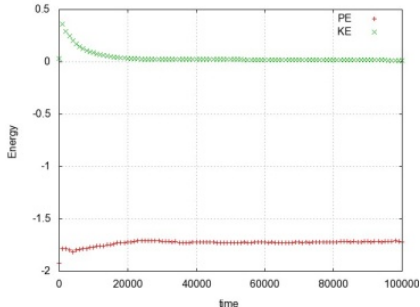

(d) Kinetic and Potential Energy vs. time

Fig. 10 Equilibration stage, $R_{H} / R_{E}=2$, Type: $A, p=0.3$ 


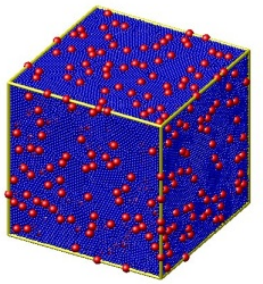

a) PM:red PE:blue

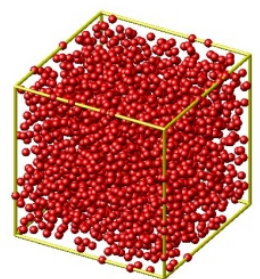

(b) PM only
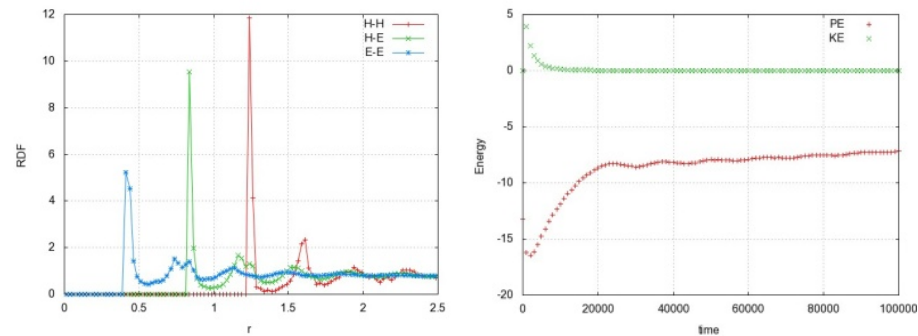

(c) $\mathrm{RDF}$

(d) Kinetic and Potential Energy vs. time

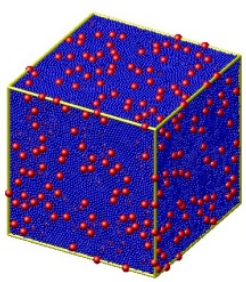

a) PM:red PE:blue

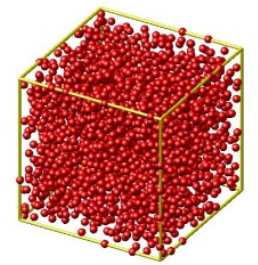

(b) PM only
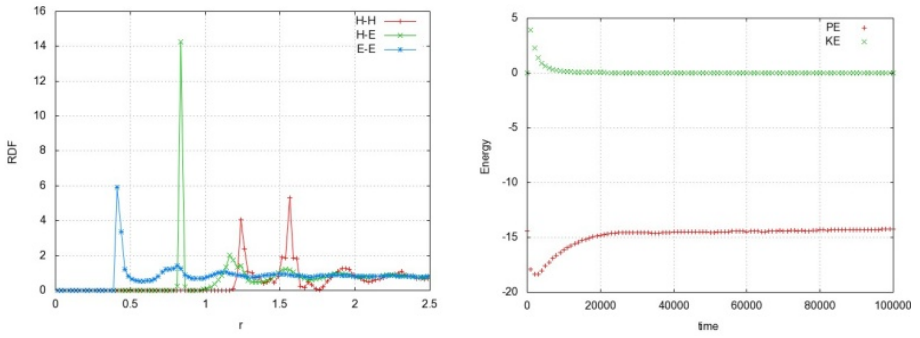

(c) $\mathrm{RDF}$

(d) Kinetic and Potential Energy vs. time

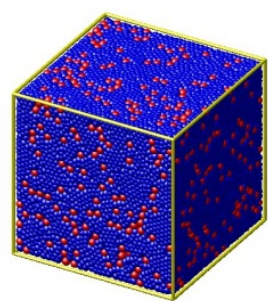

(a) PM:red PE:blue

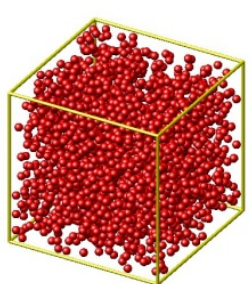

(b) PM only
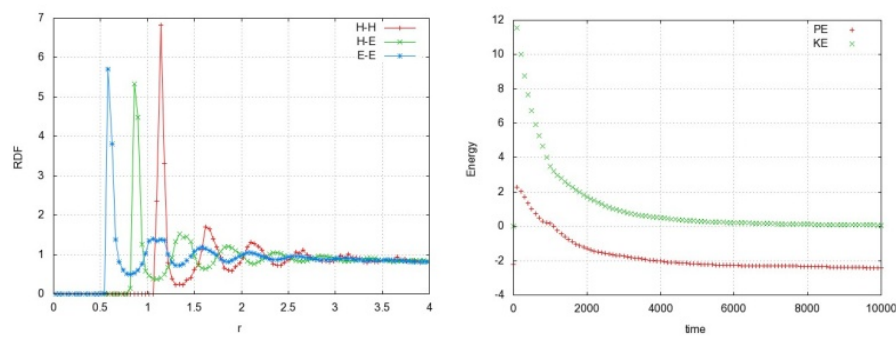

(c) $\mathrm{RDF}$

(d) Kinetic and Potential Energy vs. time

Fig. 13 Gelation stage, $R_{H} / R_{E}=2$, Type: $N, p=0.3$

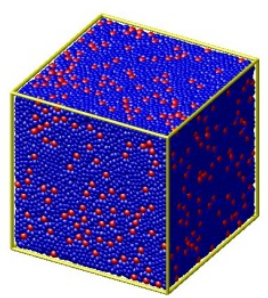

(a) PM:red PE:blue

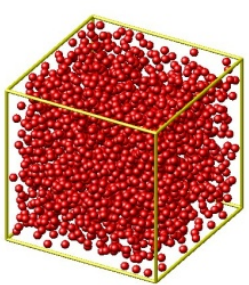

(b) PM only
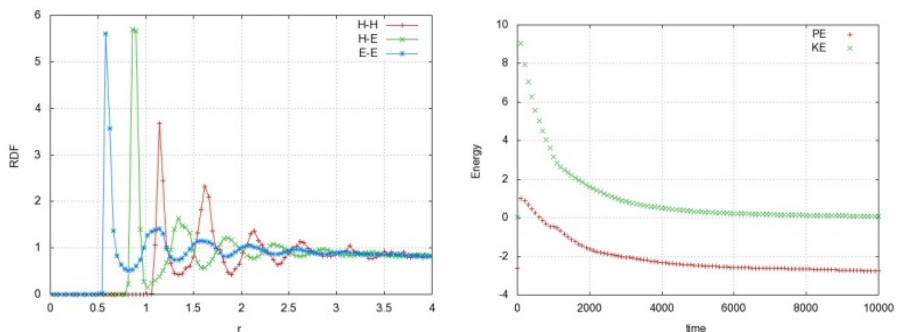

(c) RDF

(d) Kinetic and Potential Energy vs. time 


\section{Analysis}

For the mixing stage when $\mathrm{R}_{\mathrm{H}} / \mathrm{R}_{\mathrm{E}}=2$ the RDF for the particle pairs $\mathbf{H}-\mathbf{H}, \mathbf{E}-\mathbf{E}$, and $\mathbf{H}-\mathbf{E}$ are all as shown in Fig. 7 where the primary peak occurs at value $1.14 \sigma$ because there is no segregation and all particles are the same size. In the mixing stage, the packing fraction $\mathbf{f}=0.5236$ is well below the glass transition value $\mathbf{f}=0.64,0.675,0.73$ for $\mathrm{R}_{\mathrm{H}} / \mathrm{R}_{\mathrm{E}}=1,2,3$ respectively [35] [37]. Further, the potential energy in Fig. 9(d) becomes more negative to reflect the compaction of the powder [22] when the packing fraction $\mathbf{f}$ is increased.

In Fig. 7, for mixing with $\mathrm{R}_{\mathrm{H}} / \mathrm{R}_{\mathrm{E}}=2$, the $\mathrm{RDF}$ abscissa of the primary peaks of the RDF is separated to three peaks relative to the single peak in Fig. 8. In Fig. 7 the $\mathbf{H}-\mathbf{H}$ peak is at $1.14 \sigma$ and the E-E peak is at $0.58 \sigma$, which is roughly half the $\mathbf{H}-\mathbf{H}$ abscissa value. The $\mathbf{H}-\mathbf{E}$ peak is at $0.86 \sigma$ which is roughly $75 \%$ of the H-H peak location. This is due to the diameters of the E particles being one-half of the $\mathbf{H}$ particles.

In Fig. 8 for the equilibration stage for $\mathrm{R}_{\mathrm{H}} / \mathrm{R}_{\mathrm{E}}=1$, the particles are grown by instantaneously increasing the diameter by factor of $(0.64 / 0.5236){ }^{1 / 3}$, but due to the removal of the periodic boundary conditions (PBC) the effective packing fraction only changes from 0.5236 to 0.5529 . In Fig. 9 for the equilibration stage without $\mathbf{H}-\mathbf{E}$ particle affinity and where $\mathrm{R}_{\mathrm{H}} / \mathrm{R}_{\mathrm{E}}=2$, the potential energy becomes less negative indicating less compression among the spheres as a function of time. That is, the particles find their equilibrium positions because the system has not yet gelled, and this is accompanied by the kinetic energy decreasing to near zero while the temperature is lowered from $0.02 \varepsilon / \mathrm{k}_{\mathrm{B}}$ to $0.01 \varepsilon / \mathrm{k}_{\mathrm{B}}$.

To enhance H-H particle separation in Figs. 10, 12 for the equilibration stage and in Fig. 14 for the gelation stage, particle affinity is introduced between $\mathbf{H}$ and $\mathbf{E}$ with no affinity between $\mathbf{H}-\mathbf{H}$ or $\mathbf{E}-\mathbf{E}$ particles. This changes the radial distribution functions magnifying the vertical height of the $\mathbf{H}-\mathbf{E}$ peaks for all figures, although the location along the abscissa remains the same. In Figs.10, 12, and 14 the H-E primary peak is larger than the E-E peaks in the same figures because $\mathbf{H}$-E affinity introduces some attraction between $\mathbf{H}$ and $\mathbf{E}$ phases, as expected. Fig. 9 (no affinity) during equilibration with $\mathrm{R}_{\mathrm{H}} / \mathrm{R}_{\mathrm{E}}=2$ is similar to Fig. 7 (mixing) but the potential energy in Fig. 9(d) becomes less negative indicating that the particles are relaxing to equilibrium. 
In the equilibration stage, comparing Figs. 10, 12 (affinity) to Figs. 9,11 (no affinity), there is a sharp increase in the vertical height of the $\mathbf{H}-\mathbf{E}$ peak, and a relative decrease in the $\mathbf{H}-\mathbf{H}$ peak with little change in the E-E primary peak. Comparing Fig. 10 (affinity) to Fig. 9 (no affinity) where $R_{M} / R_{E}=2$, there are in Fig. 9 three distinct primary peaks, one for each inter-particle distance H-H, H-E, and E-E, but in Fig. 10 the H-E peak only is magnified due to affinity. Comparing Fig. 14 (affinity) to Fig. 13 (no affinity) for $\mathrm{R}_{\mathrm{H}} / \mathrm{R}_{\mathrm{E}}=3$, the $\mathbf{H}-\mathbf{E}$ peak shrinks by about $30 \%$ while the $\mathbf{H}-\mathbf{H}$ almost peak disappears and is distributed among numerous smaller peaks between 1 and 2 on the abscissa shifted to the right. This indicates that the $\mathbf{H}$ particles have moved away from each other, and this pattern repeats for $\mathrm{R}_{\mathrm{H}} / \mathrm{R}_{\mathrm{E}}=3$. Therefore, with affinity between the $\mathbf{H}$ and $\mathbf{E}$ particles, the $\mathbf{H}$ particles are on average farther apart from each other than they are without affinity, further supporting the results presented in Table 2. In addition, in Figs. 10, 12 (H-E particle affinity) the potential energy becomes more negative relative to Figs. 9, 11 (no affinity). This is because the extended tail of the L-J potential used to model the H-E attraction is adding more negative potential energy to the system confirming the H-E affinity. These observed relationships between figures 9 through 14 suggest that $\mathbf{E}$ particles are surrounding the $\mathbf{H}$ particles thus isolating the $\mathbf{H}$ particles from each other which support the results presented in Table 2 and Fig. 4.

\subsection{Summary}

Percolation may be interpreted as a combination of two things (a) the number of paths available at each particle node and (b) the probability of success of finding a conductive particle at each node. As the number of paths between nodes increases, the likelihood of success of a percolation from one side of the sample to the other also increases. Likewise, the probability of finding a conductive particle at any given node also increases the likelihood of a successful percolation. These two factors (success per node and paths per node) help us understand how different percolation thresholds occur.

Regarding phase and segregation, when hard shells are under pressure, segregation occurs because of the size, density, or kinetic differences among groups of particles. Liquids do not show this segregation behavior which is only seen with mixing powders [36]. Because particles will not mix as the glass 
transition is approached [36][46] , and because particles tend to agglomerate or segregate in response to pressure [36][46], growing spheres and varying the simulation box size is adopted as a way to control the undesired segregation.

When there is an attractive force between the $\mathbf{H}$ and $\mathbf{E}$ particles, the $\mathbf{H}$ particles become increasingly separated from each other, and this effect becomes more pronounced the greater the size difference between the particles. But, even without $\mathbf{H}-\mathbf{E}$ affinity when the $\mathbf{E}$ particles are smaller than the $\mathbf{H}$ particles, percolation is inhibited. Short range affinity can simulate friction to the extent that it prevents small $\mathbf{E}$ particles from segregating to interstitial locations while long range affinity promotes segregation lowering the percolation threshold.

The radial distribution functions describe inter-particle distances between particles, so when the RDF primary H-E peaks are smaller and shifted to the right, the percolation threshold is likely to increase as confirmed by the results. Our research shows two factors that can move the H-E peak to the right. The first factor is reducing the size of $\mathbf{E}$ particles relative to $\mathbf{H}$ particles to allow $\mathbf{E}$ particles to move in between and separate the $\mathbf{H}$ particles thus raising the percolation threshold. The second factor is providing short range affinity between the $\mathbf{H}$ and $\mathbf{E}$ particles which inhibits $\mathbf{E}$ particles from being expelled away from between $\mathbf{H}$ particles when the composite is compacted. These factors together raise the percolation threshold per Table 2 from $\mathrm{f}=0.1557$ to $\mathrm{f}=0.2517$ showing a relative percent increase of $61.7 \%$

\section{Conclusions}

In conclusion, we determined that the percolation threshold increases as the ratio of particles sizes $\mathrm{R}_{\mathrm{H}} / \mathrm{R}_{\mathrm{E}}$ increases. In addition, we found that the percolation threshold can be effected by inter-phase particle affinity and in fact appears to increase more so than in the cases of solely varying particle size alone. Without $\mathbf{H}-\mathbf{E}$ particle affinity, $\boldsymbol{\rho}_{\mathbf{c}}$ grows by $16.4 \%$ and $31.5 \%$ for $\mathrm{R}_{\mathrm{H}} / \mathrm{R}_{\mathrm{E}}=2$, and 3 respectively. When H-E is introduced in addition to relative particle size, the percolation threshold increases by $17.9 \%$ and $32.5 \%$ for $\mathrm{R}_{\mathrm{H}} / \mathrm{R}_{\mathrm{E}}=2$, and 3 respectively. 
In addition, we concur with other investigators [36][46][50][51][54] that complete mixing and controlling segregation is as critical to percolation as is particle compaction. In particular, we found for the purposes of particle dynamics simulation, it was necessary to mix the particles with sufficient interstitial space to allow complete mixing, but then to partially compact the particles just below glass transition to allow the particles to relax before applying final compaction to reach the target packing fractions of $64 \%, 67.5 \%$, and $73 \%$ for $\mathrm{R}_{\mathrm{H}} / \mathrm{R}_{\mathrm{E}}=1,2$, 3 respectively. This study confirms the hypothesis that both decreasing the particle size of the nonconductive phase relative to the conductive phase and some sort of affinity and or linking between the conductive and non conductive phases will maximize the electromechanical contact between the phases while minimizing the undesirable effect of electrical percolation in the conductive phase.

The results of this study were confined to a granular model where all particles were modeled as spheres and particle deformation was minimized (approximating hard-sphere conditions) with a large Leonard-Jones potential energy well depth. Therefore, future studies could involve addressing what happens when the particles deviate from spherical geometry as occurs with particle deformation caused by sintering and how friction or other adhesive forces perhaps via a colloid suspension involving other than the Leonard-Jones potential to model how a liquid solute affects the conductive phase percolation threshold in magnetoelectric composites. Further studies may involve magnetostrictive or piezoelectric materials made of polymers or the addition of a third phase of polymers or a third particle to act as a catalyst or binding agent between $\mathbf{E}$ and $\mathbf{H}$ phases.

\section{Acknowledgements}

The authors wish to express gratitude to Energy Materials Science Program (EMSE) at West Virginia University for financial and infrastructural support. In addition the authors would like acknowledge use of the West Virginia Super Computing System (Spruce Knob), funded by the National Science Foundation EPSCoR Research Infrastructure Improvement Cooperative Agreement \#1003907, without access to which the study would not have been possible. 


\section{References}

1. W. Eerenstein, N.D. Mathur, J.F. Scott (August 2006) Multiferroic and magnetoelectric materials. Nature, Dept. Matrl. Sci., Univ. Cambridge, Vol. 442(17)759:765

2. R. Grossinger, G.V. Duong, R. Sato-Turtlli (2008) The physics of magnetoelectric composites. Journal of Magnetism and Magnetic Materials 320: 1972-1977

3. J. Ma, J. Hu, Z. Li, C-W. Nan (2011) Recent progress in multiferric magnetoelectric composites: from bulk to thin films. Adv. Mater 23: 1062-1087

4. M. Bichurin, V. Petrov, S. Priya, A. Bhalla (2012) Editorial multiferric magnetoelectric composites and their applications. Adv. Cond. Matter Phys.12: 129794

5. V.L.O.de Brito, S.A.Cunha, L.V.Lemos, C.B.Nunes (2012) Magnetic properties of liquid-phase sintered $\mathrm{CoFe}_{2} \mathrm{O}_{4}$ for application in magneoelastic and magnetoelectric transducers. Sensors, 12:10086-10096

6. Y-H. Chu, L.W. Martin, M.B. Holcomb, M. Gajek, S-J. Han,Q. He, N. Balke, C-H.Yang, D.Lee, W. Hu, Q.Zhan, P-L.Yang, A.Fraile-Rodriquez, A. Scholl, S.X.Wang,R.Ramesh (2008) Electricfield control of local ferromagnetism using magnetoelectric multiferric. Nature Materials, 7:478482

7. C-W. Nan, M-I.S. Song, D. Veichland, G. Srinivasan (2008) Multiferric magnetoelectric composites: historical perspective, status, and future directions. J.Appl.Phys. 103

8. N. Ortega, A. Kumar, J.F. Scott, R.S. Katlyar (2015) Multifunctional magnetostatic materials for device applications. J.Phys: Cond .Matter 27

9. N.S.A.B. Sharif (2015) Synthesis and characterization of lead zirconate titanate $\left(\mathrm{Pb}\left[\mathrm{Zr}_{0.52} \mathrm{Ti}_{0.48}\right] \mathrm{O}_{3}\right)$ properties via high energy planetary ball milling. Ph.D. dissertation, Manufacturing Engineering, University Malaysia Pahang 
10. C.A. Randall, N. Kim, J-P. Kucera, W. Cao, T.R. Shrout (1998) Intrinsic and extrinsic size effects in fine grained morphotropic-phase-boundary lead zirconate titanete ceramics. J.Am.Cerm. 81(3):677-688

11. C-W. Nan, Y. Shen, J. Ma (2010) Physical properties of composites near percolation. Annual Review of Materials Research 40: 131-151

12. A.Bunde, W.Deiterich (2000) Percolation in composites. J. of Electroceramics, 5(2):81-92

13. T.I Muchenik, E.J. Barbero (2014) Micromechanics modeling of magnetoelectric composites. Composites and Advanced Materials CAMX Conference Proceedings, Orlando FL, Oct 13-16

14. T.I.Muchenik, E.J.Barbero (2015) Charge, voltage and work-conversion formulas for Magnetoelectric laminated composites. Smart Mater, Struct. 24

15. T.I. Muchenik (2016) Modeling of magnetoelectric composite structures. Ph.D. dissertation, West Virginia University

16. T.I. Muchenik, E.J. Barbero (2016) Prediction of extrinsic charge, voltage, and work-conversion factors for laminated magnetoelectric composites. Smart Matter. Struct. 25:015006

17. H. Scher, R. Zallen (1970) Critical density in percolation processes. J. Chem. Phys. 53:3759-3761

18. D.S. Bolintineanu, G.S. Grest, J.B. Lechman, F. Fierce, S.J. Plimpton, P.R. Schunk (2014) Particle dynamics modeling methods for colloid suspensions. Comp. Part. Mech. 1:321-356

19. L. Staron, J.C. Phillips (pub. online 23 Sept. 2015) How large grains increase bulk friction in bidisperse granular chute flows. Comp. Part. Mech., Springer

20. K.Saitoh, V.Magnanimo, S.Luding (2016) The effects of microscopic friction and size distributions on conditional probability distributions in soft particle packings. Comp. Part. Mech. (vol TBA) https://www2.msm.ctw.utwente.nl/sluding/PAPERS/2016 SaitohCPM.pdf

21. D.C.Rapaport (2014) Molecular dynamics simulation: a tool for exploration and discovery using simple models. J. Phys. Condens. Matter 26:503104-503121 
22. J. Rojek, S. Nosewicz, K. Jursak, M.Chmielewski, K. Bochenek, K. Pietrzak (2015) Discrete element simulation of powder compaction in cold uniaxial pressing with low pressure. Comp. Part. Mech. 3:513-524

23. R.P.Kusy (1977) Influence of particle size ratio on the continuity of aggregates. J. Appl. Phys. 48:5301

24. K.S. Deepa, S.K. Nisha, P. Parameswaran, M.T. Sebastian, J.James (2009) Effect of conductivity on filer on the percolation threshold of composites. Appl. Phys. Let. 94:142902

25. D. Carrera (28 Nov. 2007) Quantum tunneling in chemical reactions. MacMillan Group Meeting https://www.princeton.edu/chemistry/macmillan/group-meetings/DEC_tunneling.pdf

26. I.S. Beloborodov, A.V.Lopatin, V.M.Vinokur (2005) Coulomb effects and hopping transport in granular metals. Rev. B 72:125121

27. R.T. Hill, J.J. Mock, S.D. Wolter, N.M. Jokest, D.R.Smith, A. Chilkoti (2012) Plasmon ruler with angstrom length resolution. ACS NANO 6(10):9237-9246

28. S. Kadkhodazadeh, J.B. Wagner, H. Kneipp, K. Kneipp (2013) Coexistance of classical and quantum plasmonics in large plasmonic structures with subnanometer gaps. Applied Physics Letters 103:083103

29. J.A.Scholl, A. Garcia-Etxarri, A.L. Koh, J.A. Dionne (2013) Observations of quantum tunneling between two plasmonic nanopoles. American Chemical Society Publications, Nano Letter 13:564-569

30. J. Zhang, B.I. Shklovskii (2004) Density of states and conductivity of a granular metal or array of quantum dots. Phys. Rev. B 70:153317

31. J.Li, J-K.Kim (2007) Percolation threshold of conducting polymer composites containing 3D randomly distributed graphite nanoplatlets. Composites Science and Technology 67:2114-2120

32. X. Zhu, (Dec. 2013) Tutorial on Hertz Contact Stress. OPTI 512 https://wp.optics.arizona.edu/optomech/wp-content/uploads/sites/53/2016/10/OPTI-521-Tutorialon-Hertz-contact-stress-Xiaoyin-Zhu.pdf 
33. V. Popov (2010) Contact Mechanics and Friction. Springer-Verlag Berlin Heidelberg, file://C:/cygwin/home/WVU/DISSR/R Barbero/Papers/Springer.Contact.Mechanics.And.Frictio n.Mar.2010.eBook-ELOHiM.pdf

34. S. J. Plimpton (1995) Fast parallel Algorithms for short-range molecular dynamics. J. Comp. Phys. 117:1-19

35. G. Foffi, W. Goetz, F. Sciortino, P. Tartaglia, T. Voigtmann (2003) Mixing effects for the structural relaxation in binary hard-sphere liquids. Phys. Rev. Let. 91(8):085701

36. J.M. Ottino, D.V. Khakhar (2000) Mixing and segregation of granular materials Annual Review. Fluid Mechanics 32:55-91

37. G. Parisi, F. Zamponi (2010) Mean field theory of hard sphere glasses and jamming. Cond. Matt., PACS numbers: 05.20.-y, 61.43.Fs, 64.70.Q- https://arxiv.org/pdf/0802.2180.pdf

38. H.J.H. Brouwers (2006) Particle-size distribution and packing fraction of geometric random packing. Phys. Rev. E. 74

39. A.R. Kansal, S. Torquato, F.H. Stillinger (2002) Computer generation of dense polydisperse sphere packing. J. Chem. Phys. 117(18):8212

40. G.D. Scott, D.M. Kilgour (1969) The Density of Random Close Packing of Spheres. British Journal Applied Physics 2(2):863-866

41. J.C. GRunlan, W.W. Gerberich, L.F. Francis (2001) Lowering the percolation threshold of conductive composites using particulate polymer microstructure. J. of Applied Polymer Science 80:69-705

42. H.M. Hasanabadi, M. Wilhelm, D. Rodrigue (2014) A rheological criterion to determine the percolation threshold in polymer nano-composites, Rheol. Acta 5:869-882

43. R. Eisberg, R.Resnick (1985) Quantum mechanics of atoms molecules solids nuclei and particles. John Wiley and Sons, $2^{\text {nd }}$ Ed.

44. R.L.Kruse (1989) Programming with data structures, Chap. 17, Prentice Hall 
45. S. Nappini, Elena Magnano (2015) Surface charge and coating of $\mathrm{CeFe}_{2} \mathrm{O}_{4}$ nanoparticles evidence of preserved magnetic and electronic properties. J. Phys. Chem. C 119:25529-25541

46. K.van der Vaart, P.Gajjar, G.Epely-Chauvin, N.Andreini, J.M.N.T.Gray, C.Ancey (2015) An underlying asymmetry within particle-size segregation/. Phys. Rev. Let. 114: 238001

47. C.P. Royall, S.R.Williams, T.Ohtsuka, J.Tanaka (2008) Direct observation of a local structural mechanism for dynamic arrest. Nature Materials 7:556-561

48. T. Schilling, S. Dorosz, M. Radu, M. Mathue, S. Jungblut, K. Binder (2013) Mixtures of qnsiotropic and spherical colloids: phase behavior, confinement, percolation phenomena and kinetics. Eur. Phys. J. Special Topics 222:3039-3052

49. M-A. Suarez, N. Kem, W. Kob (2009) Out-of-equilibrium dynamics of a fractal model Gel. J. Chem. Phys. 130:194904

50. A. Amirjanov, K. Sobolev (2008) Optimization of a computer simulation model for packing of concrete aggregates. Particulate Science and Technology 26 (4):380-395

51. A.M. Puertas, M. Fuchs, M.E. Cates (2004) Dynamical heterogeneities close to a colloidal gel. J. Chem. Phys. 121(6)

52. E. Zaccarelli, S.V. Buldyrev, E. La Nave, A.J. Morene, I. Saika-Voivod, F. Sciortino, P. Tartaglia (2005) Model for reversible colloidal gelation. Phys.Rev.Let. 94:218301

53. G.S.Rohr (2001) Structure and bonding in crystalline materials. Cambridge University Press

54. M. Nakagawa, J.L. Moss, S.A. Altobelli (1999) MRI measurements and granular dynamics simulation of segregation of granular mixture. Proceedings of forth microgravity fluid physics and transport phenomena (NASA/CP-199902085526/SUPPL1)

55. D.Stauffer, A.Aharmony (1994) Introduction to Percolation Theory. $2^{\text {nd }}$ Ed. Taylor and Francis London 


\title{
Chapter 3
}

\section{Electrical percolation threshold of magnetostrictive inclusions in a piezoelectric matrix under simulated sintering conditions}

\begin{abstract}
Magnetoelectric (ME) composites can be produced by embedding magnetostrictive particles in a piezoelectric-matrix derived from a piezoelectric powder precursor. Using a bi-disperse hard-shell model [1] it has been shown that percolation in the magnetostrictive $\mathbf{H}$ phase can be reduced by both decreasing the piezoelectric $\mathbf{E}$ particle size relative to the $\mathbf{H}$ phase particle size, and increasing short range affinity between the $\mathbf{E}$ and $\mathbf{H}$ particles. This study builds on [1] by exploring the effects of deformation of the $\mathbf{H}$ or $\mathbf{E}$ particles. It was found that deformation of the $\mathbf{H}$ particles reduces the electrical percolation threshold, but that deformation of the $\mathbf{E}$ particles increases the $\mathbf{M E}$ coupling, and that both types of deformation increase the inter-phase H-E mechanical contact.
\end{abstract}

Key words: magnetostrictive, piezoelectric, magnetoelectric, particle dynamics, sintering, LAMMPS

\section{Introduction and Objectives}

Magnetostriction is a property of ferromagnetic materials that causes them to deform with strain when exposed to a magnetic field [14] [15] [16]. The piezoelectric effect is the ability to generate electrical potential in response to an applied mechanical strain [13] [14] [15] [16] [17] [18]. Magnetoelectric (ME) composites combine magnetostrictive $\mathbf{H}$ and piezoelectric $\mathbf{E}$ materials into a composite that can convert a magnetic field into an electric field or vise-versa with improved efficiency and sensitivity [17] [18] [19]. References [13] and [14] describe how to maximize the $\mathbf{M E}$ effect as a function of $\mathbf{H}$ concentration, in 
other words, maximize the piezomagnetic volume fraction $\left(\mathrm{p}=\mathrm{V}_{\mathrm{H}} /\left(\mathrm{V}_{\mathrm{H}}+\mathrm{V}_{\mathrm{E}}\right)\right.$ ) where $\mathrm{V}_{\mathrm{H}}, \mathrm{V}_{\mathrm{E}}$ are the volume of the $\mathbf{H}$ and $\mathbf{E}$ phases respectively.

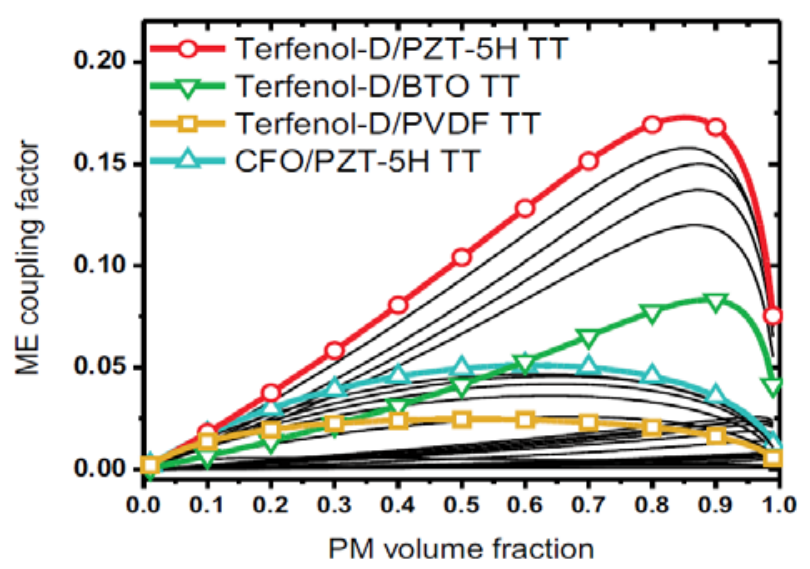

Fig. 1. Magnetoelectric coupling as a function of the relative amount of magnetostrictive material. Reprinted from [14]. Copyright (2016) with permission from IOP publishing.

As illustrated in Fig. 1, the ME coupling, which measures the efficiency of energy conversion from magnetic to electric work, increases with the piezomagetic $(\mathbf{H})$ volume fraction. However, the $\mathbf{H}$ phase is electrically conductive and its volume fraction is limited by electrical percolation because electrical conductivity diminishes the ME effect. Furthermore, deformation of the $\mathbf{H}$ particles due to sintering has a deleterious effect on the electrical percolation threshold $\left(\boldsymbol{\rho}_{\mathbf{c}}\right)$, with particles attaining the maximum percolation threshold when they are spherical. As per Fig. 1, one wishes to maximize the percolation threshold so that a larger volume of $\mathbf{H}$ phase can be used in order to maximize the ME coupling.

Sintering is a process by which powders are densified to remove interstitial space so that the packing fraction approaches one and bonds are formed between precursor particles. Sintering which leads to particle deformation without melting is done at temperatures generally below the composite melting temperature [2] [3]. Numerical techniques have been employed to study the sintering of close packed spheres [5], and numerous experimental studies have been conducted to investigate the sintering of magnetostrictive and piezoelectric ceramic composites. For example, [6] [7] [8] report the use of 
conventional ceramic processes by applying pressure with a chemical binder that promotes bonding to produce a pellet followed by applying heat below the precursor's melting temperature but sufficient to evaporate the binder. Another method reported for sintering ceramics is electric current assisted sintering (ECAS), where powders are inserted into a container that is heated by applying electric current through the powders while pressure is applied at a fixed temperature for a given period of time [9]. In yet another method, magnetoelectric PZT-CFO is produced via a chemically driven ethlenediaminetetraacetic acid (EDTA)-citrate gel process [10]. In all cases, deformation of the constituent particles occurs as a result of pressure and high temperatures or the transport of matter by viscous flow, evaporation, or atomic volume and surface migration [3] [11] [12]. Consequently, any process affected by electrical conductivity among particles in a composite must consider the relative shape of those particles which may form a conductive chain in the composite.

Aiming to exploit the as of yet unrealized ME optimization per Fig. 1, and using a hard shell model, [1] explored the relationships between particle size, inter-phase affinity and particle attraction, and the electrical percolation threshold [20] [21] in the electrical conducting magnetostrictive (H phase) when mixed with the nonconductive piezoelectric (E phase) for the purpose of maximizing the magnetoelectric (ME) effect. In [1] they concluded that the electrical percolation threshold increases when size of the $\mathbf{E}$ piezoelectric particles is reduced, and also when short range particle affinity is introduced between the $\mathbf{H}$ and $\mathbf{E}$ phases. However, the results in [1] were predicated on the assumption that neither the $\mathbf{H}$ nor $\mathbf{E}$ particles deformed from their idealized state of hard solid spheres. However, both the $\mathbf{H}$ or $\mathbf{E}$ particles may deform during sintering. Therefore, the objective of this study is to address the effect of particle deformation on the electrical percolation threshold of the $\mathbf{H}$ phase. Furthermore, it is also the objective of this study to investigate, through simulation, the likely effect of particle deformation on mechanical contact between both phases, which is needed to effectively transfer the strains from the piezomagnetic $\mathbf{H}$ phase to the piezoelectric $\mathbf{E}$ phase, which is crucial for achieving a high performance device [22]. 


\section{Methodology}

To simulate meso-scale (100 nm) particle interactions particle dynamics is performed using the Large Scale Atomic and Molecular Massively Parallel Simulation LAMMPS @ software [23]. All interactions between particles are modeled with the well known Leonard-Jones potential, and all results are expressed in Leonard-Jones natural units of $\boldsymbol{\varepsilon}$ and $\boldsymbol{\sigma}$ for energy and distance respectively.

\subsection{Simulation Specifications}

The simulation box initially contains $\mathrm{N}=(20)^{3}=$ cubic cells, each of side length $1.12246 \sigma$. Each cell contains either spherical $\mathbf{E}$ particles of diameter $1.12246 \sigma$ or 125 smaller spheres each of diameter $1.12246 \sigma / 5$ which replace an $\mathbf{H}$ particle of equal volume. A fraction $\mathrm{p}=\mathrm{N}_{\mathrm{H}} /\left(\mathrm{N}_{\mathrm{H}}+\mathrm{N}_{\mathrm{E}}\right)$ of the particles are tagged $\mathbf{H}$ for magnetostrictive while the rest a tagged as $\mathbf{E}$ for piezoelectric. Per [1] initially the hardshell simulation contains only particle modeled as spheres and is run in three stages to allow the particles to be well mixed, but also to attain a final packing fraction $\mathrm{f}=0.64$ at which random close packed particles jam or undergo a glass transition [24] [25] [26]. The first stage is the mixing (MIX) stage where particles are mixed at a low packing fraction of $f=0.5236$ (corresponding to the crystalline simple cubic lattice) to allow sufficient space for complete mixing. In the second stage, referred to as equilibration (EQU), the particle size is scaled up (by increasing $\sigma$ in the Leonard-Jones potential) to increase the packing fraction to an intermediate value where the particles are allowed to equilibrate without jamming [24]. In the third or gelation (GEL) stage, the particles are again grown so that the packing fraction reaches the jamming or glass transition [25] [26] value of $f=0.64$. After the three stages MIX, EQU, and GEL are completed, the $\mathbf{H}$ particles are replaced with deformable cubical clusters with each cube containing 125 smaller spheres (Fig. 3), and where each smaller sphere is modeled with a Leonard-Jones sigma value of $1.22246 / 5=0.22492$. The procedure is codified in a series of See Appendix C. regarding LAMMPS input scripts which are inclused in Appendix C. 
Post processing calculations for computing the coordination numbers (c.n.) and radii of gyration $\left(\mathbf{R}_{\mathbf{g}}\right)$ are performed with programs written in PERL and C included in Appendices A and B where the algorithms (dist9M.c and zapM.c) were developed and used to measure percolation between deformed $\mathbf{H}$ clusters, similar to the algorithm zap2.c used to measure hard-sphere percolation in [1], but modified to compute the smallest distance between all possible contact points between separate clusters. See Appendices A and B program for details.

\subsection{Two Dimensional Particles Dynamics}

Although this study involves full three dimensional simulations, the results of two dimensional simulations are easier to visualize, thus two dimensional simulations were initially performed to see if particle clusters diffuse around larger spherical particles in a way that mimics particle deformation that occurs during sintering. Fig. 2 illustrates that $\mathbf{H}$ clusters deform and diffuse in a way supporting the goals of this study, which is to explore the effect of particle deformation on the percolation threshold and interphase mechanical contact. It can be seen that after some time that the colored circles (H phase clusters) deform and fill the interstitial spaces between the hard blue spheres ( $\mathbf{E}$ phase). This is accomplished by slowly growing all particles in both phases in small discrete steps at the same rate until the interstitial spaces are filled and the packing fraction approaches one. The packing fraction defined as $\mathrm{f}=$ $\left(\mathrm{V}_{\mathrm{H}}+\mathrm{V}_{\mathrm{E}}\right)$ /Vbox where $\mathrm{V}_{\mathrm{H}}, \mathrm{V}_{\mathrm{E}}$, and $\mathrm{V}_{\text {box }}$ refer to the volume of the $\mathbf{H}$ phase, $\mathbf{E}$ phase, and simulation box, respectively.

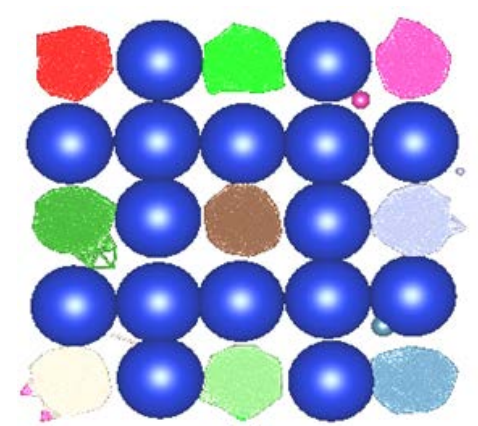

(a)

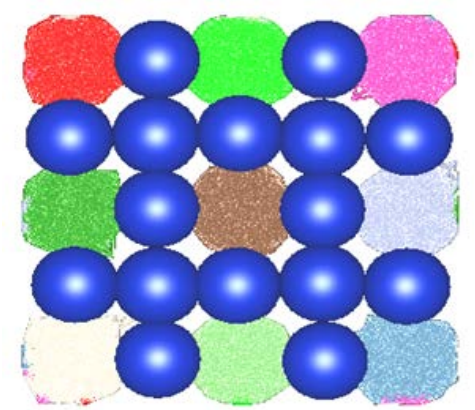

(b)

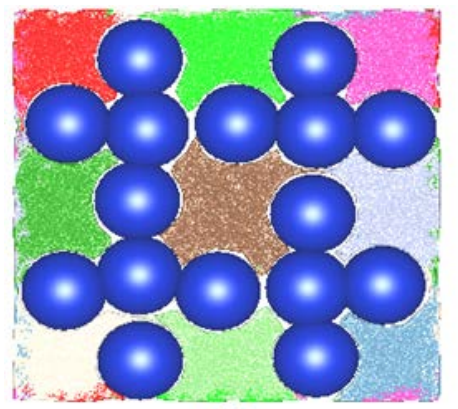

(c)

Fig. 2. 2D simulation with H-clusters deforming around undeformable spherical E-particles 


\section{Three Dimensional Particle Dynamics}

This study involves two parts. The first part is to simulate the mixing of magnetostrictive $\mathbf{H}$ and piezoelectric $\mathbf{E}$ powders, where the $\mathbf{H}$ clusters are modeled as small cubes, each composed of 125 small spheres per cube (Fig. 3). Each small sphere in a $\mathbf{H}$ cluster is modeled using the Leonard-Jones "6-12" potential, and all E particles are also modeled as hard-shell spheres using the Leonard-Jones "6-12" potential with modeling parameters $\boldsymbol{\sigma}$ and $\boldsymbol{\varepsilon}$ adjusted to reflect smaller size $($ Diameter $=1.12246 \sigma / 5)$ and interaction energy well depth $(\boldsymbol{\varepsilon})$ of the125 particles that make up each $\mathrm{H}$ cluster. The particles within each cube are allowed to move for a short time (Sect. 3.1) until the spheres are in a random configuration but still close enough to each other to constitute a cluster as shown in Fig. 4. Once the dense but random configuration of small spheres as shown in Fig. 4 is achieved, the motion is stopped (Sect. 3.1), and the collection of smaller spheres, referred to as clusters, and are regarded as equivalent to a continuous matter distribution (Fig. 5).
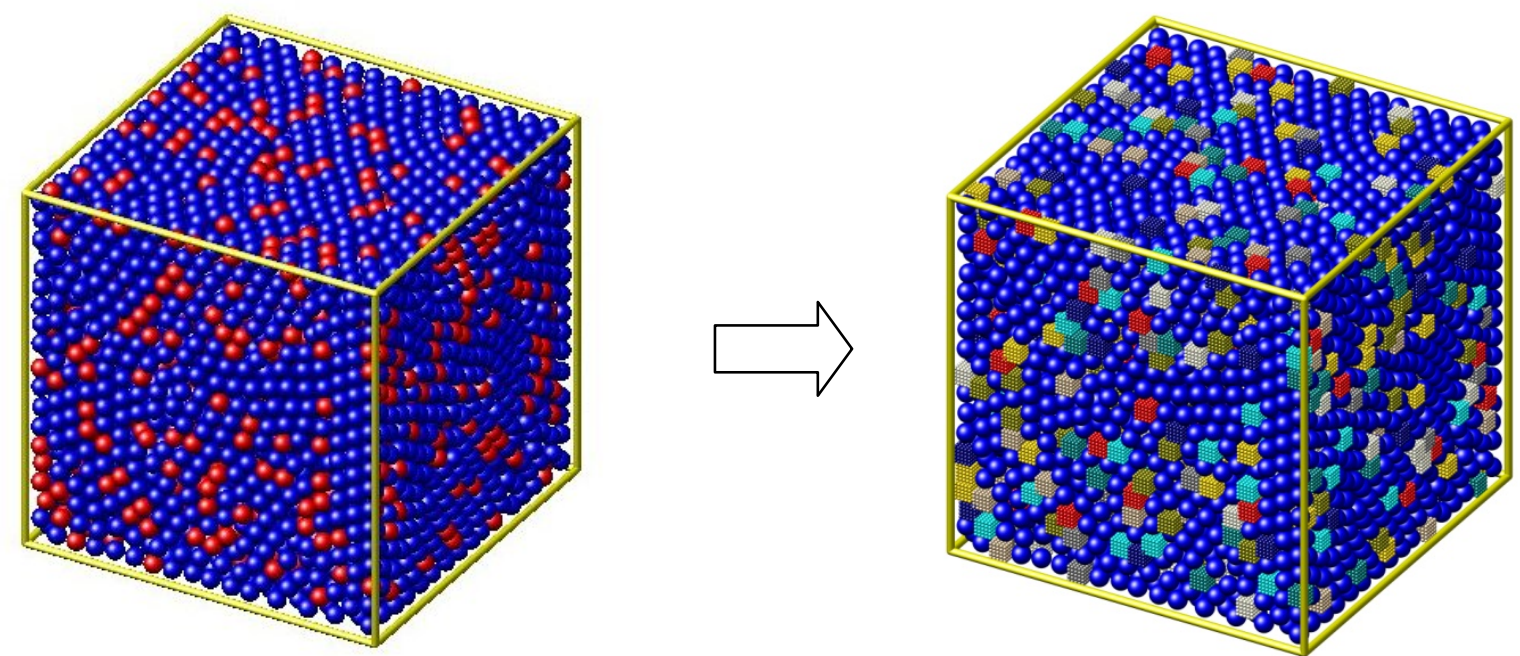

Fig. 3. Left: Hard-sphere GEL configuration for system without affinity (Noa) from [1]. Right: All Hphase red spheres on left replaced with cubical clusters (125 spheres per cluster). 


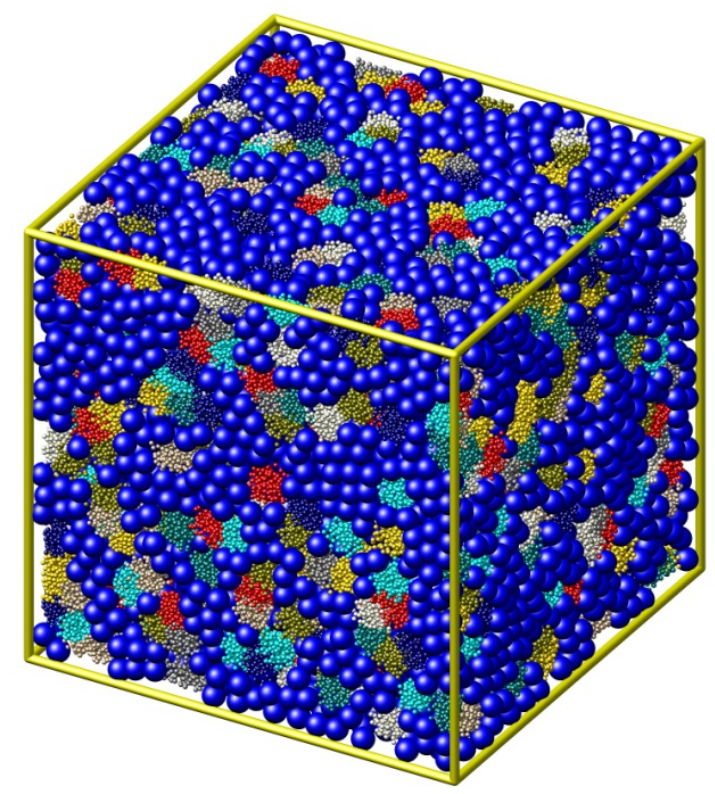

Fig. 4. $\mathbf{H}$ clusters deform and diffuse to fill the interstitial space between $\mathbf{E}$ phase spheres.
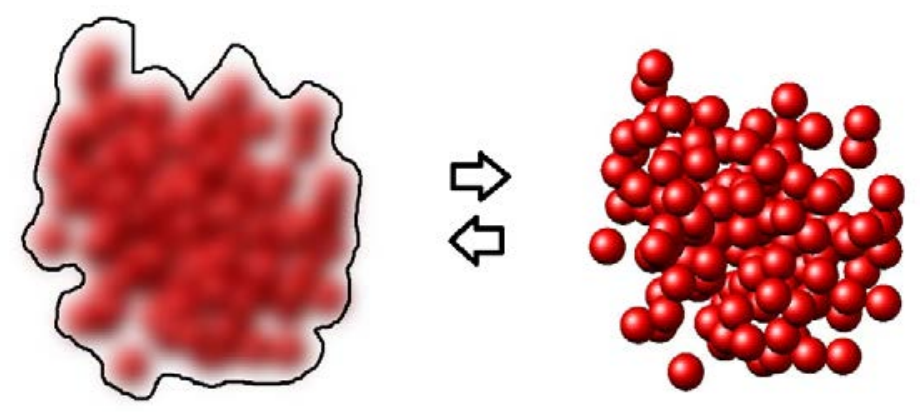

Fig. 5. Continuum of matter is replaced by 125 discrete particles in a cluster.

\subsection{Stopping Time}

During actual sintering, particle shape change (due to diffusion and surface migration) stops when the local gradient of the chemical potential between diffusing species becomes exhausted [2] [3] [4]. In addition, in actual sintering, mass transport (involving phase change) mostly occurs over small distances relative to particle size in a compacted powder mix [2]. Therefore, in the simulation particle migration should be restricted to local movement only. However, in the present simulation and taking into account that the Leonard-Jones potential represents a frictionless elastic potential, mass transport would never stop 
unless dampening or a reduction in kinetic energy are introduced. Therefore, in this study, to avoid excessive mass transport, an end-of-simulation condition is chosen to restrict the amount of medium- and long-range matter transport to under $25 \%$ of all matter in any given cluster.

In order for the clusters to simulate a continuous mass, the spheres in each cluster must be relatively close to each other. The radius of gyration is defined in Sect. 4.2 to characterize the size of the clusters. Then if any sphere in a cluster exceeds a distance greater than $2 \times \mathbf{R}_{\mathbf{g}}$ from that cluster's center of mass (Fig. 6) then for the purpose of computing percolation those small spheres are not counted as part of the continuous distribution, but are still used to compute the coordination number c.n. (Sect. 4.3). It can be seen in Table 3 that on the average the percent of small spheres outside $\mathbf{2} \times \mathbf{R}_{\mathbf{g}}$ are less than $25 \%$ for a simulation time of 3800 Leonard-Jones time steps with an iterative time step coded into LAMMPS of $\Delta \mathrm{t}=0.0002$.

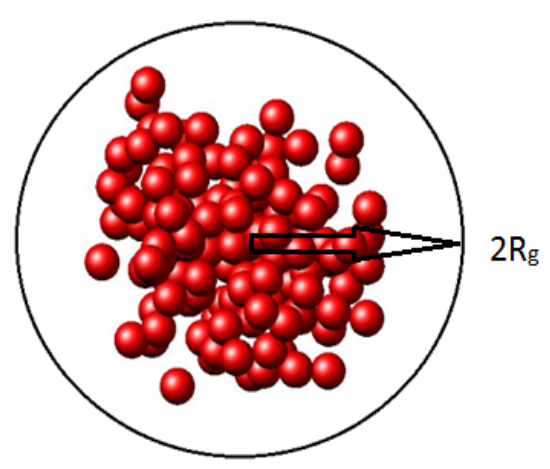

Fig. 6. Particles at a distance greater than $2 \times \mathbf{R}_{\mathbf{g}}$ from cluster center of mass are excluded from electrical percolation calculations

\subsection{Range Calculations}

A key parameter needed to compute both the intra-phase $\mathbf{H}-\mathbf{H}$ percolation and the $\mathbf{H}-\mathbf{H}$ or $\mathbf{H}-\mathbf{E}$ coordination numbers is the maximum range $\mathbf{R}_{\mathbf{m}}$ (in distance units of $\boldsymbol{\sigma}$ ) allowed between the centers of two particles such that the particles are considered connected, such that the c.n. is computed at $\mathbf{R}_{\mathbf{m}}$. There are three distinct situations for calculating range. The first (Fig. 7a.) is the electrical contact distance between $\mathbf{H}$ hard-shell spheres given by equation (1) where $\mathbf{D}$ is the diameter of $\mathbf{H}$ phase sphere = 
$1.12246 \sigma, \mathrm{SF}$ is the scaling factor $=(\mathrm{f} / 0.5236)^{1 / 3}$ per Sect. 3.2.1 and QT is the quantum tunneling factor $=(1+5 / 100)=1.05$ per Section 3.2.2.

Quantum tunneling (QT) considers the effect that classically isolated conducting particles may still be electronically connected due to the wave nature of charge carriers [28] [30]. Quantum tunneling is estimated to be $5 \mathrm{~nm}$ [27] [28] [30]. Therefore, for $100 \mathrm{~nm}$ diameter particles, the QT factor per equations (1-3) is 1.05. The scaling factor (SF) in equations (1-3) is a factor applied to sigma $(\sigma)$ in the Leonard-Jones inter-particle potential to maintain the packing fraction at the random close packed critical value of $f=0.64,0.675,0.73,0.755$ which are the particle densities above which jamming or glass transition occurs and motion becomes arrested for $\mathrm{R}_{\mathrm{H}} / \mathrm{R}_{\mathrm{E}}=1,2,3,4$ respectively [31] [32] (section 3.2.1). In this way the electrical contact range is calculated with equation (1).

The second range (Fig. 7b.) computed via equation (2) is the mechanical contact range which measure the distance between any small spheres in an $\mathbf{H}$ cluster and a nearby $\mathbf{E}$ particle. For the mechanical contact range per equation (2), no quantum tunneling is involved and the distance between the particles centers is simply 3/5 the diameter of an $\mathbf{E}$ particle.

The third range, given by equation (3), is the electrical contact distance between deformed $\mathbf{H}$-clusters. In this case, the distance between the surfaces of two $\mathbf{H}$ clusters is D×QT-D (Fig. 7c.), and the additional distance between the centers of 2 smaller spheres within each cluster is D/5. Thus the total electrical distance between $\mathbf{H}$ particle centers is $\mathrm{SF} \times[\mathrm{D} \times \mathrm{QT}-\mathrm{D}+\mathrm{D} / 5]$ scaled up by the scaling factor $\mathrm{SF}$ as explained above. Range distance between particles centers used in this study are reported in Table 1.

\subsubsection{Scaling factor SF}

For mixing, particles are sized to attain a packing fraction (f) that allows adequate room for mixing below glass transition or jamming packing fraction [24] [25]. After mixing, all particles are scaled up in size obtain the packing fraction for adequate particle deformation which corresponds to a compacted powder mix. Let $r_{1}$ be the particle radius before scaling and $r_{2}$ be the particle radius after scaling. For equally sized particles $\left(R_{H} / R_{E}=1\right)$, to increase the packing fraction to from $f=0.5236$ to $f=0.64$ for a 
fixed box volume $V_{b o x}, r_{1}$ must be increased such that $(4 / 3) \pi\left(r_{2}\right)^{3} /(4 / 3) \pi\left(r_{1}\right)^{3}=0.64 / 0.5236$. This means that $r_{2}=(0.64 / 0.5236)^{1 / 3} \times r_{1}=S F \times r_{1}$. Therefore, the scaling factor $(S F)$ for $R_{H} / R_{E}=1$ is calculated as $\mathrm{SF}=(0.64 / 0.5236)^{1 / 3}=1.069203$. In Table $1, \mathrm{SF}=(\mathrm{f} / 0.5236)^{1 / 3}$ where $\mathrm{f}$ is the packing fraction reported.

\subsubsection{Quantum tunneling}

Quantum tunneling is an effect whereby electric charge can jump a space between conductive but spacially particles which is forbidden by classical mechanics [28]. Quantum tunneling is reported to range from $3 \mathrm{~nm}$ [28] to $10 \mathrm{~nm}$ [29] between conducting particles in a non-conducting matrix. As explained in [1], if two conducting particles are at most $5 \mathrm{~nm}$ or closer, then there is a high likelihood of quantum tunneling between the two conducting particles. For $\mathbf{H}$ particle with diameter of $100 \mathrm{~nm}$, the conductivity distance used to evaluate percolation between particles centers is increased by a factor of $(100+5 / 100)=1.05$.

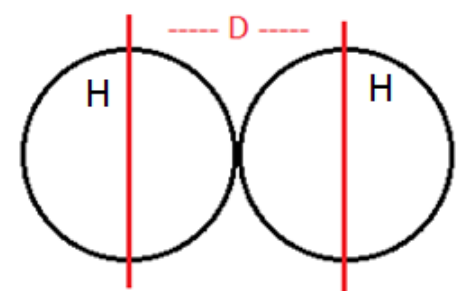

(a) $\mathrm{H}-\mathrm{H}$ electrical Equation (1)

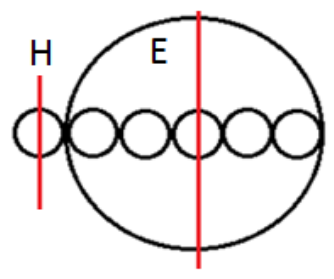

(b) H-E mechanical Equation (2)

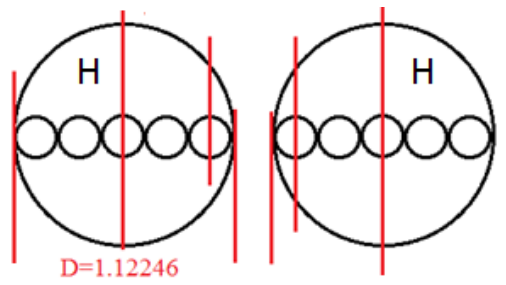

(c) H-H cluster electrical Equation (3)

Fig. 7. Illustrations for range calculations given by equations 1-3.

$$
\begin{gathered}
R_{m}=D \times S F \times Q T \\
R_{m}=3 \times \frac{D}{5} \times S F \\
R_{m}=S F \times\left(D \times Q T-D+\frac{D}{5}\right)=S F \times \frac{D}{4}
\end{gathered}
$$


Table 1. Range calculations $\left(\mathbf{R}_{\mathbf{m}}\right)$ as functions of $\mathbf{f}, \mathbf{S F}, \mathbf{Q T}$ per equations 1-3 used to compute different coordination numbers (c.n.) and the percolation thresholds $\left(\boldsymbol{\rho}_{\mathbf{c}}\right)$.

c.n. = coordination number evaluated at $\mathbf{R}_{\mathbf{m}}$

$\mathbf{f}=$ packing fraction (sect. 4.1)

SF = scaling factor (sect. 3.2.1)

$\mathbf{Q T}=$ quantum tunneling (sect. 3.2.2)

$\mathbf{R}_{\mathbf{m}}=$ range of connectivity (sect. 3.2, equations 1-3)

$\boldsymbol{\rho}_{\mathrm{c}}=\mathbf{H}-\mathbf{H}$ percolation threshold

* = See Chapter 2, Section 2.5

\begin{tabular}{|l|l|l|l|l|}
\hline parameter & $\mathbf{f}$ & $\mathbf{S F}$ & $\mathbf{Q T}$ & $\mathbf{R}_{\mathbf{m}}$ \\
\hline c.n.H-H $\left(\mathrm{R}_{\mathrm{H}} / \mathrm{R}_{\mathrm{E}}=1\right)$ & 0.64 & 1.0692 & 1.05 & $*$ \\
\hline c.n.H-H $\left(\mathrm{R}_{\mathrm{H}} / \mathrm{R}_{\mathrm{E}}=2\right)$ & 0.675 & 1.08835 & 1.05 & $*$ \\
\hline c.n.H-H $\left(\mathrm{R}_{\mathrm{H}} / \mathrm{R}_{\mathrm{E}}=3\right)$ & 0.73 & 1.11714 & 1.05 & $*$ \\
\hline c.n.H-H $\left(\mathrm{R}_{\mathrm{H}} / \mathrm{R}_{\mathrm{E}}=4\right)$ & 0.755 & 1.12975 & 1.05 & $*$ \\
\hline c.n.H-E $\left(\mathrm{R}_{\mathrm{H}} / \mathrm{R}_{\mathrm{E}}=1\right)$ & 0.64 & 1.0692 & 1 & 1.2 \\
\hline c.n.H-E $\left(\mathrm{R}_{\mathrm{H}} / \mathrm{R}_{\mathrm{E}}=2\right)$ & 0.675 & 1.08835 & 1 & 0.92 \\
\hline c.n.H-E $\left(\mathrm{R}_{\mathrm{H}} / \mathrm{R}_{\mathrm{E}}=3\right)$ & 0.73 & 1.11714 & 1 & 0.84 \\
\hline c.n.H-E $\left(\mathrm{R}_{\mathrm{H}} / \mathrm{R}_{\mathrm{E}}=4\right)$ & 0.755 & 1.12975 & 1 & 0.79 \\
\hline c.n.H-E $(\mathrm{cluster})$ & 0.64 & 1.0692 & 1 & 0.72 \\
\hline c.n.H-H $(c l u s t e r)$ & 0.64 & 1.0692 & 1.05 & 0.25 \\
\hline perc. $\boldsymbol{\rho}_{\mathbf{c}}\left(\mathrm{R}_{\mathrm{H}} / \mathrm{R}_{\mathrm{E}}=1\right)$ & 0.64 & 1.0692 & 1.05 & 1.26 \\
\hline perc. $\boldsymbol{\rho}_{\mathbf{c}}\left(\mathrm{R}_{\mathrm{H}} / \mathrm{R}_{\mathrm{E}}=2\right)$ & 0.675 & 1.08835 & 1.05 & 1.28 \\
\hline perc. $\boldsymbol{\rho}_{\mathbf{c}}\left(\mathrm{R}_{\mathrm{H}} / \mathrm{R}_{\mathrm{E}}=3\right)$ & 0.73 & 1.11714 & 1.05 & 1.32 \\
\hline perc. $\boldsymbol{\rho}_{\mathbf{c}}\left(\mathrm{R}_{\mathrm{H}} / \mathrm{R}_{\mathrm{E}}=4\right)$ & 0.755 & 1.12975 & 1.05 & 1.33 \\
\hline perc. $\boldsymbol{\rho}_{\mathbf{c}}(\mathrm{clusters})$ & 0.64 & 1.0692 & 1.05 & 0.3 \\
\hline
\end{tabular}

\subsection{Process and Thermodynamics}

All simulations of deformable phases in this study begin with $\mathbf{H}$ and $\mathbf{E}$ particles located in space as per configuration in the GEL stage of hard-shell simulations performed in [1]. One such initial configuration of $\mathbf{H}$ and $\mathbf{E}$ particles is shown on the left of Fig. 3. Next, the $\mathbf{H}$ hard spheres are replaced with cubical clusters each containing 125 smaller Leonard-Jones spheres.

It was found initially that replacing spheres with deformable cubes at the locations occupied by the precursor hard-shell $\mathbf{H}$ spheres resulted in simulation failure because portions of the cubes invaded the $\mathbf{E}$ hard-shell boundaries, causing excessive potential energy above the tollerance for the LAMMPS simulation software [23]. To solve this problem, the small spheres making up the deformable cubes were reduced in size until the cubes have time to deform into appropriate spherical clusters. The small spheres 
were then allowed to equilibrate for a short period of time, and then they were slightly scaled up in size by increasing the Leonard-Jones distance parameter $\boldsymbol{\sigma}$ and adjusting the energy parameter $\boldsymbol{\varepsilon}$. The system was then allowed to equilibrate. This process of sudden but incremental scaling followed by short time equilibration was repeated until all particles were at the required size to restore the packing fraction ( $\mathrm{f}=$ $\left.\mathrm{V}_{\mathrm{E}}+\mathrm{V}_{\mathrm{H}} / \mathrm{V}_{\text {box }}\right)$ and volume ratio $\mathrm{p}=\mathrm{V}_{\mathrm{H}} /\left(\mathrm{V}_{\mathrm{E}}+\mathrm{V}_{\mathrm{H}}\right)$ to the same values they had before the $\mathbf{H}$ particles were replaced with deformable cubes, where $\mathrm{V}_{\mathrm{H}}$ and $\mathrm{V}_{\mathrm{E}}$ equal the total volume taken up by the $\mathrm{H}$ and $\mathrm{E}$ phases respectively [1]. Energy fluctuation during the simulation of particle deformation is shown in Fig. 8. The potential energy (PE) is discontinuous because the size of all particles both $\mathbf{H}$ and $\mathbf{E}$ are increased suddenly at each discontinuity. This increase in PE causes a corresponding increase in kinetic energy (KE) as shown Fig. 8.

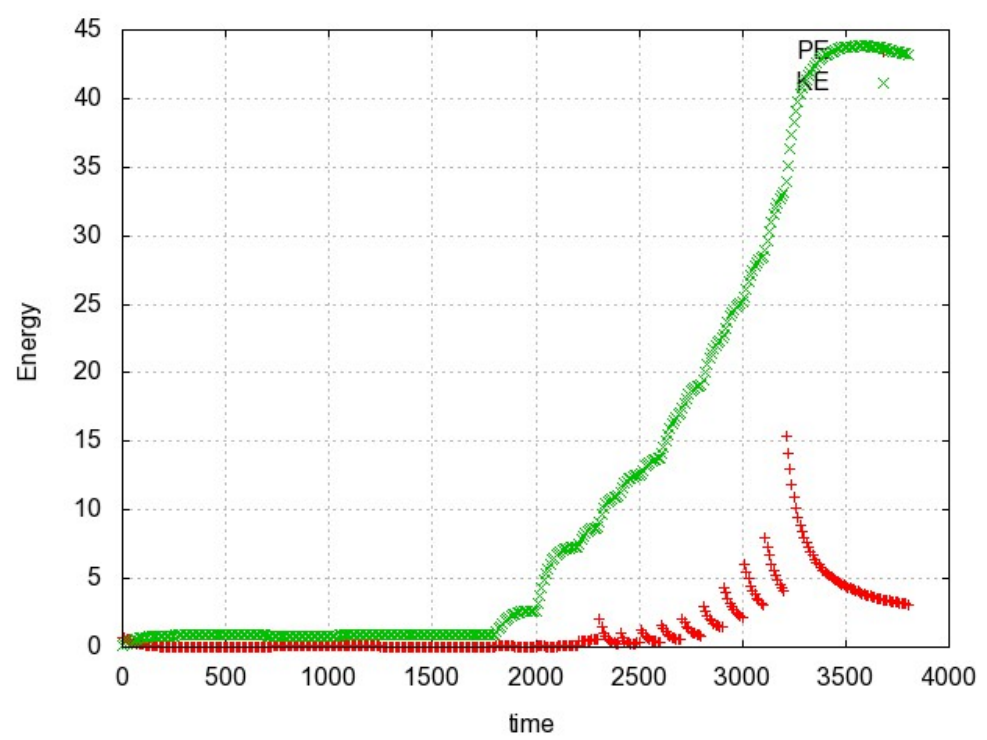

Fig. 8. “ $\times ”$ is Kinetic Energy “+” is Potential Energy

\section{Characterization and Measurement}

The techniques used for characterization and measurement of particle deformation and inter-phase mechanical contact are described in this section.

4.1 Packing fraction 
The packing fraction (f) is the volume of all solid matter relative to the volume of the simulation box.

$$
f=\frac{V_{E}+V_{H}}{V_{\text {box }}}
$$

The volume ratio ( $\boldsymbol{\rho}$ ) is the volume of the $\mathrm{H}$ phase relative to the volume of the simulation box.

$$
\rho=\frac{V_{H}}{V_{\text {box }}}=f \times p
$$

The volume fraction (p) is the volume of the $\mathrm{H}$ phase relative to the volume of all the solid matter.

$$
p=\frac{V_{H}}{V_{H}+V_{E}}
$$

\subsection{Radius of Gyration}

The radius of Gyration $\left(\mathbf{R}_{\mathbf{g}}\right)$ is the distance representing the effective rotational inertia of a distribution of point masses located at distances $r_{\mathbf{i}}$ with respect to the distribution's center of mass given by equation (7) located at $\mathrm{r}_{\mathrm{cm}}$ [33]

$$
R_{g}=\sqrt{\frac{1}{n} \sum_{i=1}^{n}\left(r_{i}-r_{c m}\right)^{2}}
$$

In this study, the radius of gyration is used to represent the elongated shape of the clusters as they deviate from the spherical shape. By way of comparison, the radius of gyration of $\mathbf{H}$ and $\mathbf{E}$ perfect spheres with diameter $2 \times \mathrm{R}=1.12246 \sigma$ is $\mathbf{R}_{\mathrm{g}}=0.775 \times \mathrm{R}=0.435 \sigma$ ( $\sigma$ is a natural unit of distance in LeonardJones units). A cylinder of radius $\mathbf{R}$ and equal volume to an $\mathbf{E}$ or $\mathbf{H}$ sphere has a radius of gyration such that $\left(\mathbf{R}_{\mathbf{g}}\right)^{2}=\mathrm{R}^{2} / 2+(4 / 3 \mathrm{R})^{2} / 12$, so that $\mathbf{R}_{\mathbf{g}}=0.452 \sigma$. It can be seen that the $\mathbf{R}_{\mathbf{g}}$ of a cylinder is larger than the $\mathbf{R}_{\mathbf{g}}$ of a sphere of the same volume. Thus, $\mathbf{R}_{\mathbf{g}}$ provides an indication of a cluster's deformation during the simulation pseudo-sintering (the effect of particle deformation on the percolation threshold during sintering).

\subsection{Coordination Number}

The coordination number (c.n.) [34] [36] [37] [38] [39] for a given particle is the number of contacts it has with other particles. The average c.n. is the average coordination number of all particles in a given 
class. In crystallography [36], the lattice structure of a simple cubic lattice has a c.n. $=6$, a body centered cubic lattice has a c.n. $=8$, and the hexagonal closest packed has c.n. $=12$. In this study, we define two types of coordination numbers. The first, CNHH (left Fig. 9), is the number of contacts the average $\mathbf{H}$ particle make with other $\mathbf{H}$ particles. The other type of coordination number is labeled CNHE (right Fig. 9) which is the number of contacts the average $\mathbf{H}$ particle makes with other $\mathbf{E}$ particles.

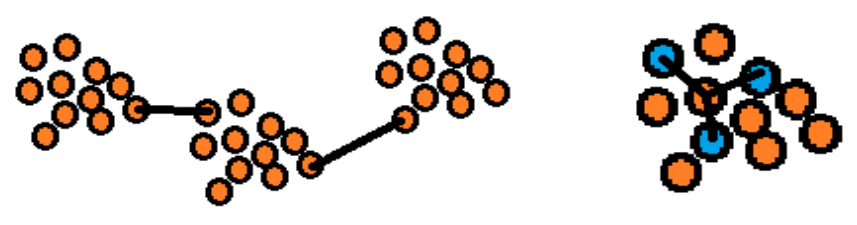

Fig. 9. Illustration of intra-phase CNHH (left), and inter-phase CNHE (right) coordination.

Per Fig. 9, CNHH characterizes the percolation paths an electrical signal could follow through the $\mathbf{H}$ phase. Thus the higher the CNHH, the more likely the percolation threshold $\left(\boldsymbol{\rho}_{\mathbf{c}}\right)$ will decrease. CNHE characterizes the mechanical contact between the $\mathbf{H}$ and $\mathbf{E}$ phases, which is proportionate to the efficiency of mechanical energy transfer between the $\mathbf{H}$ and $\mathbf{E}$ phases. All small spheres belonging to a particular cluster may participate in the calculation for either coordination number regardless of their distance from the center of mass of the cluster. The average c.n. for random close packed configurations of perfect spheres of equal size is reported to be approximately six [34] [38]. Higher c.n. values provide an indication of a lower percolation threshold and higher ME mechanical contact.

\section{Results}

\subsection{Deformation of $\mathbf{H}$ Phase Around Rigid E Phase}

This section focuses on the effects of the deformation of $\mathbf{H}$ particles around rigid spherical $\mathbf{E}$ particles. In Table 2, "RU” in the left most column stands for the initial configuration of particles used in this study taken after the GEL stage is completed in [1]. "Noa” stands for "No affinity” between any particles used to compute the initial GEL configuration per [1]. “Adh” stands for “Affinity” between $\mathbf{H}$ and $\mathbf{E}$ phase particles used to compute the initial GEL configuration per [1]. " $\boldsymbol{\rho}_{\mathbf{c}}$ " represents the critical or 
smallest volume ratio, $\boldsymbol{\rho}=\mathbf{V}_{\mathbf{H}} /\left(\mathbf{V}_{\text {box }}\right)$ for which electrical $\mathbf{H}-\mathbf{H}$ percolation is predicted. $\mathbf{V}_{\mathbf{H}}$ is the volume of the $\mathbf{H}$ phase and $\mathbf{V}_{\text {box }}$ is the total volume of the simulation box which for this study which is $(20 \times 1.12246 \sigma)^{3} . \mathbf{R}_{\mathbf{g}}$ is the radius of gyration in Leonard-Jones units of sigma $\sigma$.

Furthermore in Tables 2 and 3, “Bonds” stands for the number of bonds between deformed H particles found during percolation. "CNHH” stands for the coordination number of all $\mathbf{H}$ particles with respect to other $\mathbf{H}$ particles when all particles are modeled as hard-shell spheres with $\mathbf{R}_{\mathbf{H}} / \mathbf{R}_{\mathbf{E}}=1$ using data from [1]. CNHE1 through CNHE4 stands for the coordination number of all $\mathbf{H}$ particles with respect to surrounding $\mathbf{E}$ particles for $\mathbf{R}_{\mathbf{H}} / \mathbf{R}_{\mathbf{E}}=1,2,3,4$ respectively. It can be seen in Table 2 that the sum of CNHH and CNHE1 is approximately six which confirms the average coordination number of random close packed spheres of equal size [34] [37] [38]. Also in Table 2, it can also be seen that for the no interparticle (Noa) cases, the inter-phase $\mathbf{H}-\mathbf{H}$ coordination $\mathrm{CNHH}$ is directly proportional to the percolation threshold $\boldsymbol{\rho}_{\mathbf{c}}$. That is, as the $\mathbf{H}-\mathbf{H}$ c.n. goes up or down, so does the $\mathbf{H}-\mathbf{H}$ electrical percolation threshold. It can also be seen that the inter-phase particle coordination numbers CNHE1 to CNHE4 increases sharply with increasing difference in particle size $R_{H} / R_{E}=1$ to 4 .

Table 2. Characterization of hard-shell spheres simulation

\begin{tabular}{|c|c|c|c|c|c|c|c|c|}
\hline & $\underline{\mathbf{Q}}_{\mathrm{c}}$ & $\underline{\mathbf{R g}}$ & Bonds & $\underline{\mathrm{CNHH}}$ & CNHE1 & CNHE2 & CNHE3 & CNHE4 \\
\hline RU8/Noa & 0.25 & 0.435 & 1802 & 2.07 & 3.84 & 17.74 & 39.18 & 59.11 \\
\hline RU9/Noa & 0.26 & 0.435 & 1450 & 2.21 & 4.19 & 17.16 & 38.8 & 56.37 \\
\hline RU10/Noa & 0.22 & 0.435 & 1245 & 1.74 & 4.45 & 18.36 & 44.72 & 60.75 \\
\hline AVG & 0.243 & 0.435 & 1499.000 & 2.007 & 4.160 & 17.753 & 40.900 & 58.743 \\
\hline STD & 0.021 & 0.000 & 281.714 & 0.241 & 0.306 & 0.600 & 3.314 & 2.213 \\
\hline cov & 8.555 & 0.000 & 18.793 & 12.026 & 7.358 & 3.380 & 8.102 & 3.767 \\
\hline RU8/Adh & 0.3 & 0.435 & 1653 & 1.68 & 4.77 & 18.01 & 39.44 & 60.82 \\
\hline RU9/Adh & 0.3 & 0.435 & 1931 & 1.73 & 4.86 & 19.33 & 39.29 & 59.72 \\
\hline RU10/Adh & 0.29 & 0.435 & 1626 & 1.63 & 4.88 & 18.3 & 40.27 & 58.59 \\
\hline AVG & 0.297 & 0.435 & 1736.667 & 1.680 & 4.837 & 18.547 & 39.667 & 59.710 \\
\hline STD & 0.006 & 0.000 & 168.838 & 0.050 & 0.059 & 0.694 & 0.528 & 1.115 \\
\hline $\operatorname{cov}$ & 1.946 & 0.000 & 9.722 & 2.976 & 1.211 & 3.740 & 1.331 & 1.867 \\
\hline
\end{tabular}


The effects of $\mathbf{H}$ particle deformation are reported in Table 3. “A00/\#” stands for no affinity between any particles, and “A12/\#” stands for affinity between the $\mathbf{H}$ and $\mathbf{E}$ phase during the particle deformation. In all simulations, no H-H or E-E affinities are introduced, and \# stands for run number. All runs were repeated three times. The term “ \%strys” stands for the percent of small spheres per cluster that have drifted away more than $2 \times \mathbf{R}_{\mathbf{g}}$ from the center of mass of a given cluster, which is used to stop the simulation at a preset value to avoid excessive long range mass transport. "CNHH-M" stands for the average coordination number of all $\mathbf{H}$ clusters with respect to other $\mathbf{H}$ clusters. "CNHE-M" stands for the average coordination number of all $\mathbf{H}$ clusters with respect to all neighboring $\mathbf{E}$ particles.

Table 3. Characterization of deformable H-clusters in simulation

\begin{tabular}{|c|c|c|c|c|c|c|}
\hline Noa & $\rho_{c}$ & $\underline{\mathbf{R g}}$ & Bonds & \%strys & CNHH-M & CNHE-M \\
\hline $\mathrm{A} 00 / 1$ & 0.14 & 0.594 & 716 & 22.05 & 8.91 & 15.41 \\
\hline $\mathrm{A} 12 / 1$ & 0.14 & 0.597 & 715 & 19.29 & 8.81 & 17.72 \\
\hline$A 00 / 2$ & 0.17 & 0.59 & 986 & 25.15 & 9.59 & 16.42 \\
\hline$A 12 / 2$ & 0.17 & 0.593 & 990 & 22.52 & 9.53 & 19.02 \\
\hline$A 00 / 3$ & 0.13 & 0.593 & 512 & 20.77 & 9.18 & 14.03 \\
\hline$A 12 / 3$ & 0.11 & 0.595 & 281 & 18.3 & 9.52 & 15.63 \\
\hline AVG & 0.143 & 0.594 & 700.000 & 21.347 & 9.257 & 16.372 \\
\hline STD & 0.023 & 0.002 & 274.693 & 2.457 & 0.341 & 1.777 \\
\hline cov & 16.312 & 0.394 & 39.242 & 11.509 & 3.682 & 10.855 \\
\hline$\underline{\text { Adh }}$ & $\underline{p}$ & $\underline{\mathbf{R g}}$ & Bonds & \%strys & CNHH-M & CNHE-M \\
\hline $\mathrm{A} 00 / 1$ & 0.15 & 0.588 & 792 & 24.75 & 8.28 & 16.55 \\
\hline $\mathrm{A} 12 / 1$ & 0.15 & 0.592 & 719 & 15.54 & 8.16 & 18.32 \\
\hline$A 00 / 2$ & 0.17 & 0.59 & 986 & 25.15 & 9.59 & 16.42 \\
\hline $\mathrm{A} 12 / 2$ & 0.17 & 0.593 & 990 & 25.52 & 9.53 & 19.02 \\
\hline$A 00 / 3$ & 0.14 & 0.589 & 754 & 15.54 & 8.6 & 15.42 \\
\hline$A 12 / 3$ & 0.14 & 0.592 & 755 & 12.68 & 8.49 & 17.21 \\
\hline AVG & 0.153 & 0.591 & 832.667 & 19.863 & 8.775 & 17.157 \\
\hline STD & 0.014 & 0.002 & 122.523 & 5.879 & 0.628 & 1.323 \\
\hline cov & 8.910 & 0.333 & 14.715 & 29.597 & 7.152 & 7.713 \\
\hline
\end{tabular}

It can be seen in Table 3 that the percolation threshold for systems with H-E particle affinity (Adh) up to the GEL stage (Table 3, bottom half) is higher than the percolation threshold for systems with no affinity (Noa). This reinforces the conclusion that H-E particle affinity increases the $\mathbf{H}-\mathbf{H}$ percolation 
threshold regardless of $\mathbf{H}$ particle deformation. It may be seen in Table 3 that both the coordination CNHH-M and CNHE-M values have a relatively low spread about their average values. Furthermore, the coordination number for the “A00” (No inter-phase affinity during deformation) and “A12” (H-E affinity during deformation) are very similar while the $\mathbf{R}_{\mathbf{g}}$ values are almost the same in all cases. This indicates that when particle deformation (represended by $\mathbf{R}_{\mathbf{g}}$ ) is used to limit the sintering time (section 3.1), the percolation threshold is relatively independent of how the particles reached their final geometrical state , i.e., particle clusters are independent of path taken in phase-space to arrive at their asymmetrical conformations in space.

A comparison between the results of Tables 2 and 3 is presented in Table 4 where average values and their associated coefficients of variance (COV) from Tables 2 and 3 are reported. It can be seen in Table 4 that regardless of whether the $\mathbf{H}$ phase deformation is started from the hard-shell affinity (Adh) or nonaffinity (Noa) hard-shell configurations [1], the percolation threshold ( $\left.\boldsymbol{\rho}_{\mathbf{c}}\right)$ drops from 0.243 to 0.143 (Noa conditions), and from 0.297 to 0.153 (Adh conditions) as the radius of gyration $\left(\mathbf{R}_{\mathbf{g}}\right)$ increases from $\left(\mathbf{R}_{\mathbf{g}}=\right.$ $0.435 \sigma$ ) for a perfect sphere to values of $\mathbf{R}_{\mathbf{g}}$ between $0.591 \sigma$ to $0.594 \sigma$ for clusters of small asymmetrical spheroids (Fig. 10).

In Fig. 11 a representative histogram of $\mathbf{R}_{\mathbf{g}}$ values is shown for a case of no-affinity (Noa) at the hardshell GEL stage [1], followed by no-affinity (A00) particle deformation in this study. It can be seen that the majority of clusters deform about the same, as indicated by the sharp peak of $\mathbf{R}_{\mathbf{g}}$ in Fig.11. 
Table 4. Comparison of Tables 2 and 3.

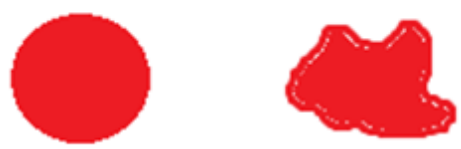

\begin{tabular}{|c|c|c|c|}
\hline Noa & $\begin{array}{l}\text { No sintering } \\
\text { Hard-shell }\end{array}$ & $\begin{array}{l}\text { Pseudo-sintering } \\
\text { Deformation }\end{array}$ & Comments \\
\hline $\boldsymbol{\rho}_{\mathrm{c}}$ & $0.243(8.55) \rightarrow$ & $0.143(16.31)$ & Perc. threshold decreases \\
\hline $\mathbf{R}_{\mathrm{g}}$ & $0.435 \quad \rightarrow$ & $0.594(0.394)$ & Sphere deforms \\
\hline $\mathrm{CN}_{\mathrm{H}-\mathrm{H}}$ & $2.01(12.03) \rightarrow$ & $9.26(3.68)$ & Gain mechanical contact \\
\hline $\mathrm{CN}_{\mathrm{H}-\mathrm{E}}$ & $4.16(7.36) \rightarrow$ & $16.37(10.86)$ & Gain mechanical contact \\
\hline c.n. & $6.17 \quad \rightarrow$ & 25.63 & \\
\hline \%strys & & $21.35(11.51)$ & \\
\hline
\end{tabular}

\begin{tabular}{|c|c|c|c|}
\hline Adh & $\begin{array}{l}\text { No sintering } \\
\text { Hard-shell }\end{array}$ & $\begin{array}{l}\text { Pseudo-sintering } \\
\text { Deformation }\end{array}$ & Comments \\
\hline$\rho_{\mathrm{c}}$ & $0.297(1.95) \rightarrow$ & $0.153(8.91)$ & Perc. threshold decreases \\
\hline $\mathbf{R}_{\mathrm{g}}$ & $0.435 \quad \rightarrow$ & $0.591(0.33)$ & Sphere deforms \\
\hline $\mathrm{CN}_{\mathrm{H}-\mathrm{H}}$ & $1.68(2.98) \rightarrow$ & $8.78(7.15)$ & Gain mechanical contact \\
\hline $\mathrm{CN}_{\mathrm{H}-\mathrm{E}}$ & $4.94(1.21)$ & $17.16(7.71)$ & Gain mechanical contact \\
\hline c.n. & 6.62 & 25.94 & \\
\hline \%strys & & $19.83(29.60)$ & \\
\hline
\end{tabular}




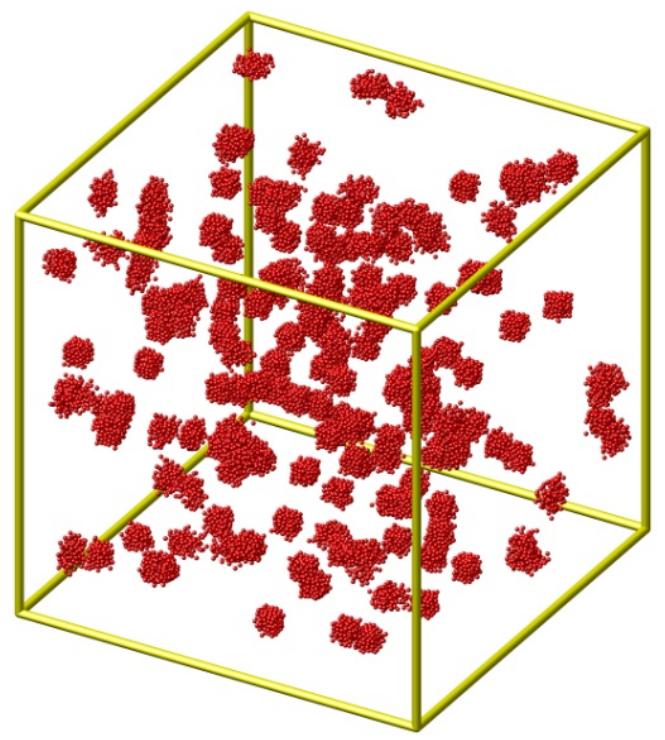

Fig. 10. Cube clusters morph into spheroids.

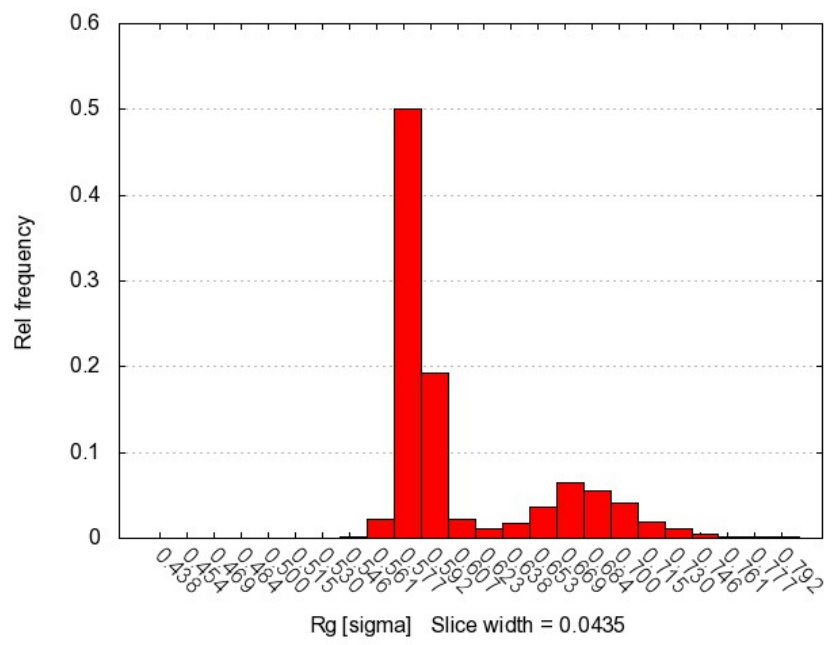

Fig. 11. Histogram of radius of gyration $\left(\mathbf{R}_{\mathbf{g}}\right)$ under Noa followed by A00 conditions.

Per Table 4, it can be seen that the coordination number for hard shells without particle deformation $(\mathrm{CNHH})$ have values between the values of 6 to 7 in approximate agreement with others who have studied the coordination of random close packed spheres [34] [37] [38]. This is true for both no-affinity (Noa) and affinity (Adh) conditions. Also per Table 4, when $\mathbf{H}$ phase particle deformation is allowed, $\mathbf{H}$ H coordination increases from a value approximately 2 (CNHH hard-shell model) to values in the range 8 
to 9 (CNHH-M pseudo-sintering model). This indicates that the number of paths for electrical signal across the $\mathbf{H}$ phase has increased dramatically due to elongation of the clusters, and this increase in electrical percolation paths explains the reduction of $\mathbf{H}-\mathbf{H}$ electrical percolation reported in Table 4, column 2. Also per Table 4, CNHE (c.n. between the $\mathbf{H}$ and $\mathbf{E}$ phases) increases from approximately 4 to values in the range 16 to 17 . This is a fourfold increase in mechanical contact between the $\mathbf{H}$ and $\mathbf{E}$ phases that has occurred when the $\mathbf{H}$ particles are deformed. This is an encouraging finding as the optimum ME composite would be one that maximizes the $\mathbf{H}$-E mechanical contact while minimizing $\mathbf{H}$ H electrical percolation, maximizing the percolation threshold.

\subsection{Deformation of E Phase Around Rigid $\mathbf{H}$ Phase}

This section focuses on the effects of deformation of $\mathbf{E}$ particles around rigid spherical $\mathbf{H}$ particles. Since the $\mathbf{E}$ phase constitutes the majority of the $\mathbf{M E}$ composite's volume, it is not necessary to collect the $\mathbf{E}$ particles into small clusters. The Scher-Zallen [35] invariant percolation threshold for a conducting phase $(\mathbf{H})$ of spheres immersed in equally sized insulting $(\mathbf{E})$ spheres is $\rho=0.154$. Barbero and Bedard [1] found that decreasing the size of the $\mathbf{E}$ phase to $R_{H} / R_{E}=3$ could increase the percolation threshold to $\boldsymbol{\rho}_{\mathbf{c}}=0.297$ or 0.243 depending on whether H-E particle affinity (Adh) is considered or not, respectively. In the affinity (Adh) case, at most $29.7 \%$ of the volume of the ME composite that does not percolate is composed of $\mathbf{H}$ phase. Therefore, we assume that immersing larger spherical $\mathbf{H}$ particles in a random close packed continuum of smaller $\mathbf{E}$ particles is equivalent to deforming $\mathbf{E}$ particles to fill the interstitial space between rigid $\mathbf{H}$ particles. Results of simulation with the $\mathbf{H}$ and $\mathbf{E}$ hard-shell spheres as in [1] are shown in Fig. 12.

In Fig. 12, the increase in the percolation threshold $\boldsymbol{\rho}_{\mathbf{c}}$ versus the decrease in the size of the $\mathbf{E}$ phase is apparent. However, somewhere between $\mathrm{R}_{\mathrm{H}} / \mathrm{R}_{\mathrm{E}}=3$ to 4 , the percolation threshold tapers off in the case of no affinity (Noa) between the $\mathbf{H}$ and $\mathbf{E}$ phases, and decreases for the case where the $\mathbf{H}$ and $\mathbf{E}$ phases experience short range affinity (Adh). In light of decreases in percolation thresholds for both Noa and Adh cases, we postulate that the increase in the percolation threshold $\left(\boldsymbol{\rho}_{\mathbf{c}}\right)$ is due to the smaller particles 
moving to interstitial space between the $\mathbf{H}$ particles and hence separating the $\mathbf{H}$ particles away from each other. However, when the $\mathbf{E}$ phase particles become too small, they can no longer push the $\mathbf{H}$ particles apart. In addition, the deviation from a in the linear relationship between $\boldsymbol{\rho}_{c}$ and $R_{H} / R_{E}$ between 3 and 4 observed in Fig. 12 becomes more pronounced when there is H-E (Adh) affinity between the $\mathbf{H}$ and $\mathbf{E}$ particles. This is because when two large $\mathbf{H}$ particles are both attracted to the same $\mathbf{E}$ particle, they are effectively attracted to each other, decreasing the distance between $\mathbf{H}$ phase particles, and thus decreasing the percolation threshold. The pair-wise Leonard-Jones potential used in this model does not have any friction or lateral force components, so this decrease in the trend of increasing percolation threshold $\left(\boldsymbol{\rho}_{\mathrm{c}}\right)$ versus $R_{H} / R_{E}$ shown in Fig. 12 may not be so severe in practice because actual particles will experience frictional forces that may keep the smaller $\mathbf{E}$ particles from being pushed out of the way. In the case where there is $\mathbf{H}-\mathbf{E}$ particle affinity (Adh), because the L-J potential does not have any lateral force components, the $\mathbf{E}$ particles in this simulation can still be pushed into interstitial spaces between $\mathbf{H}$ particles and act as attractors within the $\mathbf{H}$ phase, closing the distances between $\mathbf{H}$ particles thus lowering the percolation threshold.

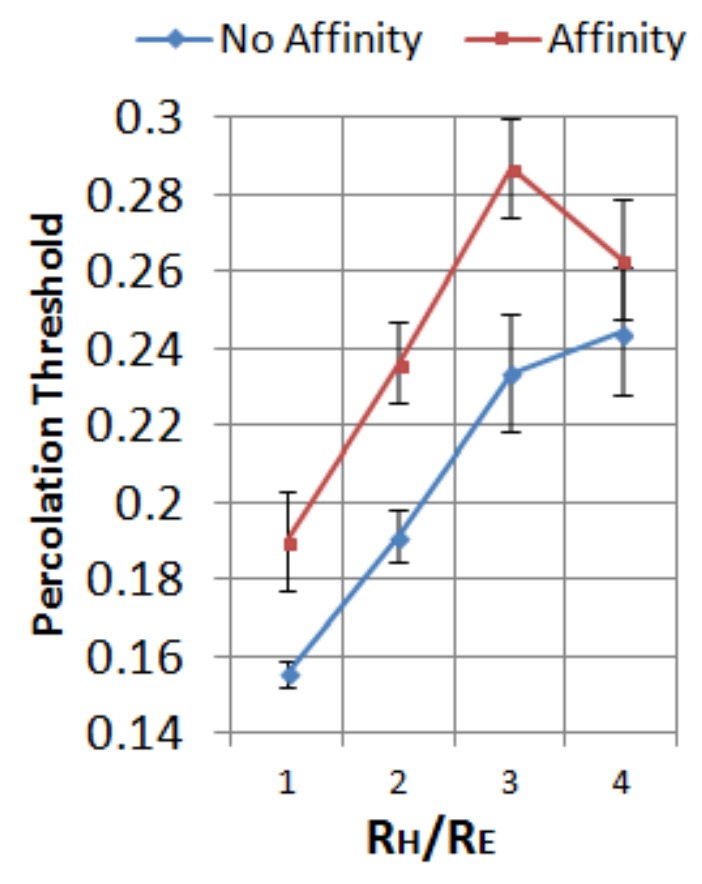

Fig. 12. Percolation threshold when both $H$ and $E$ phases are modeled as hard-shell spheres. 


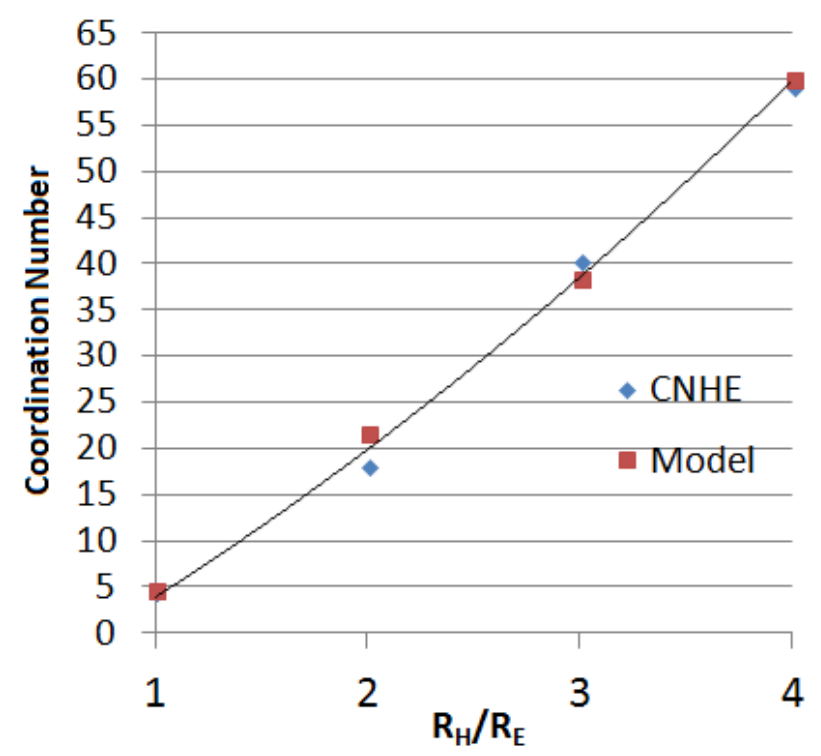

Fig. 13. CNHE1-4 H-E inter-phase coordination number of hard-shell model.

As previously mentioned, the coordination number c.n. is an indicator of mechanical contact because the higher the c.n., the larger the numbers of contacts between particles and thus the more efficient the energy transfer. The predicted relationship between inter-phase ME mechanical contact (CNHE) and relative particle size $R_{H} / R_{E}$ is shown in Fig. 13. When the relative size of the $\mathbf{E}$ particles decreases with respect to the $\mathbf{H}$ particles the number of bonds between the $\mathbf{E}$ and $\mathbf{H}$ particles (CNHE) clearly increases. Therefore, we propose a simple qualitative parametric model (Fig. 14) that purports that mechanical contact between $\mathbf{H}$ and $\mathbf{E}$ particles is of the same order of magnitude as the ratio of the surface area of a larger sphere centered at the $\mathbf{H}$ particle intersecting the cross sectional area of the smaller $\mathbf{E}$ particles at the E particle's center. Equation (8) is parametrically adjusted to fit the c.n. data per Fig. 13, where in equ. (8) $\mathbf{D}=$ the diameter of the $\mathbf{H}$ particles, and $x=R_{H} / R_{E}$ the ratio of the $\mathbf{H}$ to $\mathbf{E}$ particle radii. Fig. 14 illustrates the derivation of equ. (8) where the c.n. $\times$ intersect area of the small sphere $=$ the area of the larger sphere at distance $\mathrm{R}_{\mathrm{H}}+\mathrm{R}_{\mathrm{E}}$. 


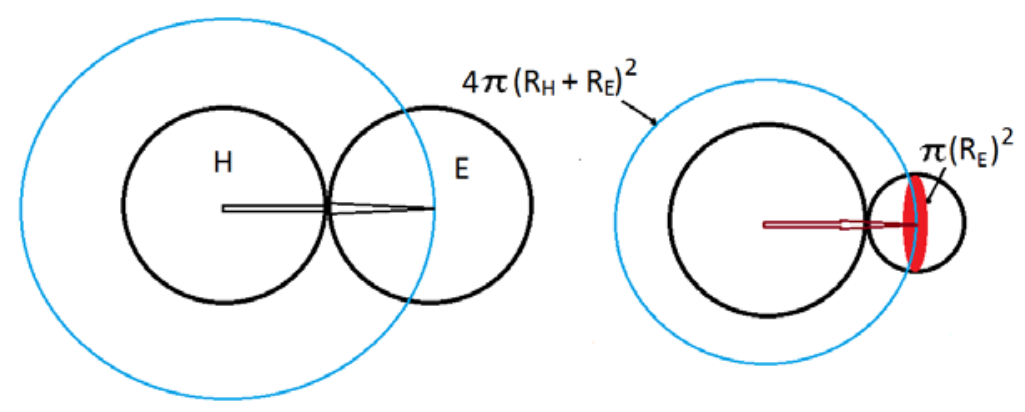

Fig. 14. Two equal size particles in contact (left), and two unequal sized particles in contact (right).

$$
\text { c. } n . \propto 4 \pi \frac{\left(\frac{D}{2}+\frac{D}{2 x}\right)^{2}}{\pi\left(\frac{D}{2 x}\right)^{2}}=4(x+1)^{2} \approx 2.4 \times\left(R_{H} /_{R_{E}}+1\right)^{2}-5
$$

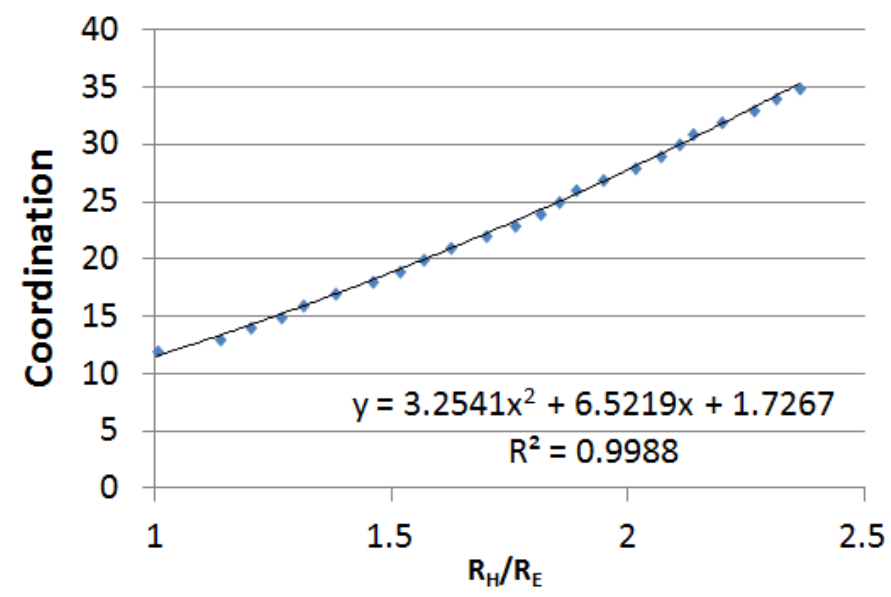

Fig. 15. Polynomial curve fit of [44] Table 2.

Liang [44 Table 2] produced a table of coordination numbers for a bi-disperse population of spheres as a function of relative particle size. Curve fitting their results of coorination versus relative particle size shows a good fit for a quadratic polynomial validating our results in Fig. 13. 


\section{Conclusions}

In conclusion, we observed that the deformation of the electrically conducting, magnetostrictive $\mathbf{H}$ phase particles, which occurs in practice due to sintering, is likely to lower the percolation threshold in bidisperse $\mathbf{M E}$ composites because the deformed particles will be elongated (Higher $\mathbf{R}_{\mathbf{g}}$ ) and will also have more contact points (higher c.n.). Hence any electric signal will have a greater number of paths to travel across a magnetoelectric composite, thus diminishing the ME effect.

We also observed that for spherical particles the inter-phase H-E coordination number (CNHE) is likely to increase quadratically as the piezoelectric $\mathbf{H}$ phase particles deform during sintering. However, allowing the $\mathbf{H}$ phase particles to deform during sintering may counteract the $\mathbf{M E}$ enhancing effect of reducing E-phase relative particle size and thus lower the percolation threshold. The simulation data suggest that the best way to raise the electrical percolation threshold $\boldsymbol{\rho}_{\mathbf{c}}$ while maintaining a maximum mechanical ME coupling between the $\mathbf{E}$ and $\mathbf{H}$ phases is perhaps to use piezoelectric $\mathbf{E}$ particles at (3.5) ${ }^{-1}$ the size of magnetostrictive $\mathbf{H}$, and promote $\mathbf{E}$ and $\mathbf{H}$ particle affinity whenever possible. Furthermore, during sintering, it seems better to promote deformation of the $\mathbf{E}$ phase while minimizing deformation of the $\mathbf{H}$ phase in order to maximize mechanical coupling and the electrical percolation threshold $\left(\boldsymbol{\rho}_{\mathbf{c}}\right)$.

We also observed that the radius of gyration $\left(\mathbf{R}_{\mathbf{g}}\right)$ on average grows from $\mathbf{R}_{\mathbf{g}}=0.435 \sigma$ for perfectly spherical particles to an average value of $\mathbf{R}_{\mathbf{g}}=0.592 \sigma$ in all cases. The simulation stops at less than $25 \%$ mass transport from each $\mathrm{H}$ particles center of mass in all cases but it also arrives at a the same increase in $\mathbf{R}_{\mathbf{g}}$ of $(0.5925-0.435) / 0.435 \times 100=6.85 \%$ for the same simulation run time (3800 time steps), implying a connection between sintering time and average extent of particle deformation.

Regarding pre-sintering particle mixing, numerous studies including [40] [41] [42] [43] report the phenomena of phase segregation when mixing particles of different sizes or mass, which is highly deleterious to the percolation threshold. Therefore, it is required that the $\mathbf{H}$ and $\mathbf{E}$ phases be well mixed with each other prior to sintering. In addition, we confirmed the results of [34] [38] and others that the coordination number for random close packed hard-shell spheres is approximately between 5.9 and 6.7, and we propose a simplified qualitative model which predicts that the coordination number between bi- 
disperse spheres increases quadratically as a function of relative particles size $\left(x=R_{H} / R_{E}\right)$ between the two species.

\section{Acknowledgements}

The authors wish to acknowledge use of the West Virginia Super Computing System (Spruce Knob), funding by the National Science Foundation EPSCoR Research Infrastructure Improvement Cooperative Agreement \#1003907, without access to which this study would not have been possible. We also acknowledge the guidance and assistance from Dr. X. Song, Dr. Y. Chen, Dr. F.A. Campo, and Dr.

E.M. Sosa at West Virginia University’s department of Mechanical and Aerospace Engineering. 


\section{References}

1. E.J. Barbero, A.J. Bedard (2017) Electrical percolation threshold of magnetostrictive inclusions in a piezoelectric matrix composite as a function of relative particle size. Computational Particle Mechanics (published 25 July online ISSN 2196-4378)

2. J.K. Mackenzie, R. Shuttleworth (1949) A phenomenological theory of sintering. Proceeding of the Physical Society B 62(12):833-852

3. C. Herring (1950) Effect of change of scale in sintering phenomena. Journal of Applied Physics 21:301-303

4. L.C. DeJonghe, M.N. Rahaman (2003) Handbook of Advanced Ceramics, Chapter 4.1 Sintering of Ceramics, Elsevier, Academic Press, ISBN 0-12-654640-1

5. R. Bjork, V. Takare, H.L. Frandsen, N. Pryds (2012) The sintering behavior of close packed spheres. Scripta Materialia 67(1):81-84

6. W. Zhang, R.E. Eitel (2011) Low-temperature sintering and properties of 0.98PZT-0.02KN ceramics with LiBiO2 and CuO addition. J. Am. Ceram. Soc. 94(10):3386-3390

7. X. Chao, D. Ma, R. Gu, Z. Yang (2010) Effects of CuO addition on the electrical responses of the low-temperature sintered $\mathrm{Pb}\left(\mathrm{Zr}_{0.52} \mathrm{Ti}_{0.48}\right) \mathrm{O}_{3}-\mathrm{Pb}\left(\mathrm{Mg}_{1 / 3} \mathrm{Nb}_{2 / 3}\right)-\mathrm{Pb}\left(\mathrm{Zb}_{1 / 3} \mathrm{Nb}_{2 / 3}\right) \mathrm{O}_{3}$ ceramics. Journal of Alloys and Compounds 491:698-702

8. G. Srinivasan, E.T. Rasmussen, J. Gallegos, R. Srinivasan (2001) Magnetoelectric bilayer and multilayer structures of magnetostrictive and piezoelectric oxides. Physical Review B 64:214408

9. R. Orru, R. Licheri, A.M. Locci, A. Cincotti, G. Cao (2009) Consolidation/synthesis of materials by electric current activated/assisted sintering. Materials Science and Engineering 63:127-287

10. L. Weng, Y. Fu, S. Song, J. Tang, J. Li (2007) Synthesis of lead zirconate titanate-cobalt ferrite magnetoelectric particulate composites via and ethlenediamineetetraacetic acid-citrate gel process. Scripta Materialia 56:465-468 
11. K.M. Allen, N. Auyeung, N. Rahmatian, J.F. Klausner, E.N. Coker (2013) Cobalt Ferrite in YSZ as use as reactive material in solar thermochemical water and carbon dioxide splitting, part II: kinetic modeling. J. of Minerals, Metals \& Materials Society 65(12):1682-1693

12. S. Hara, A. Ohi, N. Shikazono (2015) Sintering analysis of sub-micron-sized nickel powders: kinetic monte carlo simulation verified by FIB-SEM reconstruction. Journal of Powder Sources 276:105-112

13. T.I. Muchenik, E.J. Barbero (2015) Charge, voltage and work conversion formulas for magnetoelectric laminated composites. Smart Matter Structure 24:1-15

14. T.I. Muchenik, E.J. Barbero (2016) Prediction of extrinsic charge, voltage, and work-conversion factors for laminated magnetoelectric composites. Smart Matter Structure 25:1-16

15. W.Eerenstein, N.D.Mathur, J.F.Scott (2006) Multiferroic and magnetoelectric materials. Nature 442:759-765

16. R. Grossinger, G.V. Duong, R. Sato-Turtlli (2008) The physics of magnetoelectric composites. Journal of Magnetism and Magnetic Materials 320:1972-1977

17. J.Ma, J.Hu, Z.Li, C-W.Nan (2011) Recent progress in multiferric magnetoelectric composites: from bulk to thin films. Adv. Matter 23: 1062-1087

18. C-W.Nan, M.I. Bichurin, D.Veichland, G.Srinivasan (2008) Multiferric magnetoelectric composites: historical perspective status and future directions. J. Appl. Phys. 103:031101

19. M.Bichurin, V.Petrov, S.Priya, A.Bhalla (2012) Editorial multiferric magnetoelectric composites and their applications. Adv. Cond. Matter Phys.12: 129794

20. A. Bunde, W. Deiterich (2000) Percolation in composites. J. Electroceramics 5(2):81-92

21. C-W. Nan, Y. Shen, J. Ma (2010) Physical properties of composites near percolation. Annu. Rev. Matter. Res 40:131-151

22. E.J. Barbero (Editor) (2016) Multifunctional Composites. CreateSpace, Charleston NC ISBN:978-15168-0452-8

23. S.J.Plimpton (1995) Fast parallel algorithms for short-range molecular dynamics. J. Comp. Phys. $117: 1-19$ 
24. A. Donev, S. Torquato, F.H. Stillinger, Robert Connelly (2004) Jamming in hard sphere and disk packings. J. Applied Physics 95(3):989-999

25. A.R. Kansal, S. Torquato, F.H. Stillinger (2002) Computer generation of dense polydisperse sphere packings. J. Chem. Phys. 117:8212-8218

26. E. Zaccarrelli, S.V. Buldyrev, E. La Nave, A.J. Moreno, I. Saika-Voivod, F. Sciortino, P. Tartagelia (2005) Model of reversible colloidal gelation. Phys. Rev. Lett. 94:218301

27. I.S. Beloborodov, A.V. Lopatin, V.M. Vinkur (2005) Coulomb effects and hopping transport in granular metals. Physical Review B 72:125121

28. D. Carrera (2007) Quantum tunneling in chemical reactions. MacMillian Group 28 November. https://www.princeton.edu/chemistry/macmillan/group-meetings/DEC tunneling.pdf

29. J. Li, J-K. Kim (2007) Percolation threshold of conducting polymer composites containing 3D randomly distributed graphite nanoplatelets. Composites Science and Technology 67:2114-2120

30. S. Kadkhodazadeh, J.B.Wagner, H.Kneipp, K.Kneipp (2013) Coexistance of classical and quantum plasmonics in large plasminix structures with subnanometer gaps. Applied Physics Letters 103:083103:1-4

31. M.D. Rintoul, S. Torquato (1996) Computer simulations of dense hard-sphere systems. J. Chem. Phys. 105(20):4198-4201

32. P. Jalali, M. Li (2004) An estimate of random close packing density in monodisperse hard spheres. J. Chem. Phys. 120:1138-1139

33. Radius of Gyration Research Gate 21 October 2014 https://www.researchgate.net/file.PostFileLoader.html?id=5824372493553baba73427c1\&assetKey= $\underline{\text { AS\%3A426799843811331\%401478768420695 }}$

34. A. Bezrukov, D. Stoyan, M. Bargeil (2001) Spatial statistics for simulated packings of spheres. Image Anal. Stereol. 20:203-206

35. Scher, R. Zallen (1970) Critical density in percolation processes. J.Chem. Phys. 53:3759-3760

36. G.S. Rohr (2001) Structure and bonding in crystalline materials. Cambridge University Press 
37. S.S. Tulluri (2003) Analysis of random packing of uniform spheres using the monte-carlo simulation method. Masters Thesis, New Jersey Inst. of Tech., Dept. of Mech. Eng.

38. J. Zhou, Y. Zhang, J.K. Chen (2009) Numerical simulation of random packing of spherical particles for powder-based additive manufacturing. Journal of Manufacturing Science and Engineering. 131:031004:1-8

39. R. Richard, L. Oger, J.P. Troadec, A. Gervios (2001) A model of binary assemblies of spheres. Eur. Phys. J. E 6:295-303

40. J.M. Ottino, D.V. Khakhar (2000) Mixing and segregation of granular materials. Annu. Rev. Fluid Mech. 32:55-91

41. S.W. Meier, R.M. Lueptow, J.M. Ottino () A dynamical system approach to mixing and segregation of granular materials in tumblers. Advances in Physics 56(5):757-827

42. D.C. Rapaport (2014) Molecular dynamics simulation: a tool for exploration and discovery using simple models. J. Phys. Condensed Matter 26:503104:1-17

43. K.van der Vaart, P. Gajjar, G. Epley-Chauvin, N. Andreini, J.M.N.T. Gray, C. Ancey (2015) Underlying Asymetry within particle size segregation. Phys. Rev. Lett. 114:238001:1-5

44. H. Liang, W. Wang (1985) Coordination numbers for rigid spheres and local compositions. Fluid Phase Equilibria. 24:165-175 


\section{Chapter 4}

\section{Extension of hard-shell methods from Chapter 2 applied to relative particle size of $\mathrm{R}_{\mathrm{H}} / \mathrm{R}_{\mathrm{E}}=1,2,3,4$}

\subsection{Introduction}

This chapter describes what happens when the particle dynamics hard-shell methods in Chapter 2 are applied to two cases. The first case is a continuation of the results per Chapter 2, but with the particle size ratio of $R_{H} / R_{E}=4$. The second case is where the $E$ phase is divided into four equally sized subgroups, and each subgroup is at different particle size ranging from $R_{H} / R_{E}=1$ to 4 .

\subsection{Review of Chapter 2.}

In chapter 2 it was reported that at when the size of the piezoelectric $\mathbf{E}$ phase particles is reduced relative to the magnetostrictive $\mathbf{H}$ particles, the electrical intra-phase $\mathbf{H}-\mathbf{H}$ percolation threshold ( $\boldsymbol{\rho}_{\mathbf{c}}$ ) increases per Fig. 1. It can be seen in Fig. 1 (repeated here from Fig. 4 Ch.2) that $\boldsymbol{\rho}_{\mathrm{c}}$ increases linearly under no particles affinity conditions (Noa) and under H-E particle affinity conditions (Adh), but that the percolation threshold $\left(\boldsymbol{\rho}_{\mathbf{c}}\right)$ has approximately a $25 \%$ increase relative to the no affinity (Noa) data. 


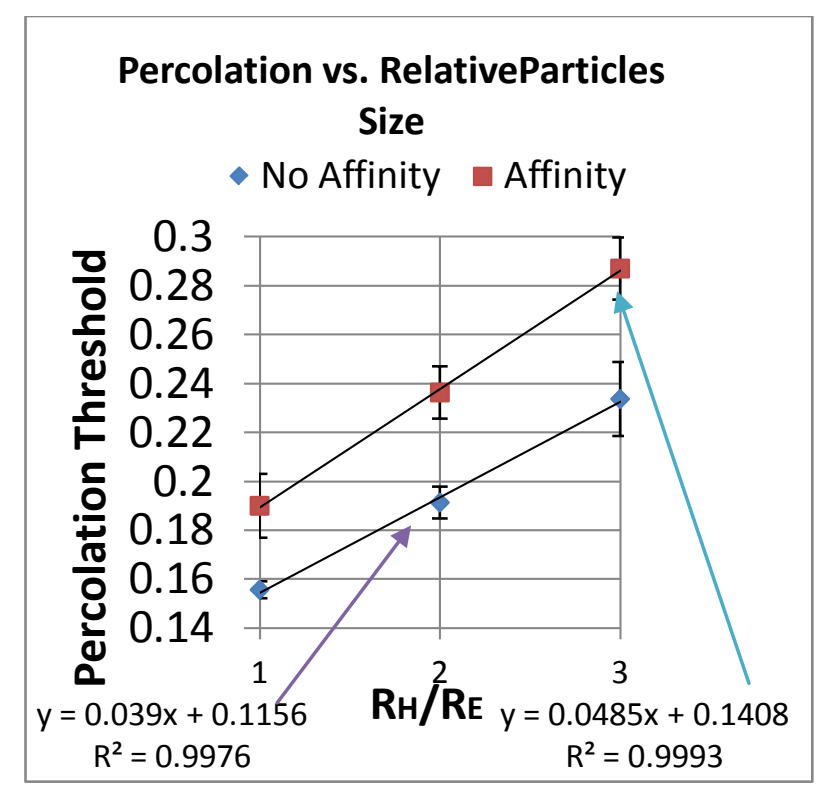

Fig. 1. Percolation threshold $(\rho c)$ versus particle size

3.0 Methods and results for $\mathrm{R}_{\mathrm{H}} / \mathrm{R}_{\mathrm{E}}=4$

\subsection{Methods}

The three stages MIX, EQU, and GEL used in Chapter 2 for $\mathrm{R}_{\mathrm{H}} / \mathrm{R}_{\mathrm{E}}=1,2,3$ where in the MIX stage the all particles are mixed at a low packing fraction of $\mathrm{f}=0.5236$ for one million time steps from a temperature of $0.01 \varepsilon / \mathrm{k}_{\mathrm{B}}$ to $0.02 \varepsilon / \mathrm{k}_{\mathrm{B}}$, then for nine million additional times at a constant temperature of $0.2 \varepsilon / \mathrm{k}_{\mathrm{B}}$ with periodic boundary conditions on (Appendix C). In the subsequent EQU stage, the periodic boundary conditions are changed to reflective (momentum is reversed upon impact), and the inter-particle Leonard-Jones potential distance parameter sigma $(\sigma)$ is increased so that the packing fraction is $f=$ $0.755 \times(20 / 21)^{3}=0.652$. In the final GEL stage, the interaction between the walls and the particles is changed from reflective to Leonard-Jones walls so that the particles are compressed and the packing fraction is thus increased to $\mathrm{f}=0.755$ corresponding to the jamming packing fraction (Chapter 2 ). In both the EQU and GEL stages the temperature is ramped from 0.02 to $0.01 \varepsilon / \mathrm{k}_{\mathrm{B}}$ to allow only the effects from potential energy and stress between particles to determine final particle position. In addition to the afore procedure described above (which is identical to Chapter 2) the following adjustments were made for simulations where $\mathrm{R}_{\mathrm{H}} / \mathrm{R}_{\mathrm{E}}=4$. The EQU stage was broken up into two 50 million time step blocks. In 
the first block the mass of the $\mathbf{H}$ particles is 100 Leonard-Jones units, and the mass of the $\mathbf{E}$ particle is 20.169 L-J. This is followed by a second 50 million time step block where the mass of the $\mathbf{H}$ particles is 100 but the mass of the $\mathbf{E}$ particles is 2.169 which is the correct relative mass of $\mathbf{H}$ to $\mathbf{E}$ based on size and molar weight. This was done to slow down the smaller $\mathbf{E}$ particles for part of the run until the particles could equilibrate sufficiently, otherwise due to their small size the E particles would acquire velocities outside the tolerance levels of the LAMMPS software and fail. The GEL stage is similarly broken up into two ten-thousand time step block where the mass of the $\mathbf{E}$ particles is increased by a factor of 10 in the first block and returned to its correct relative mass by size and molar weight relative to $\mathbf{H}$ in the second block (Appendix C).

\subsection{Results}

The question next is what happens if $\mathrm{R}_{\mathrm{H}} / \mathrm{R}_{\mathrm{E}}$ is increased to 4 ? The answer surprisingly is not a direct extension of the trend in Fig. 1. Rather it can be seen in Fig. 2 that there is a dip in the trend per Fig. 1, and this dip is more severe under $\mathbf{H}-\mathbf{E}$ affinity (Adh) conditions than with no affinity (Noa) conditions.

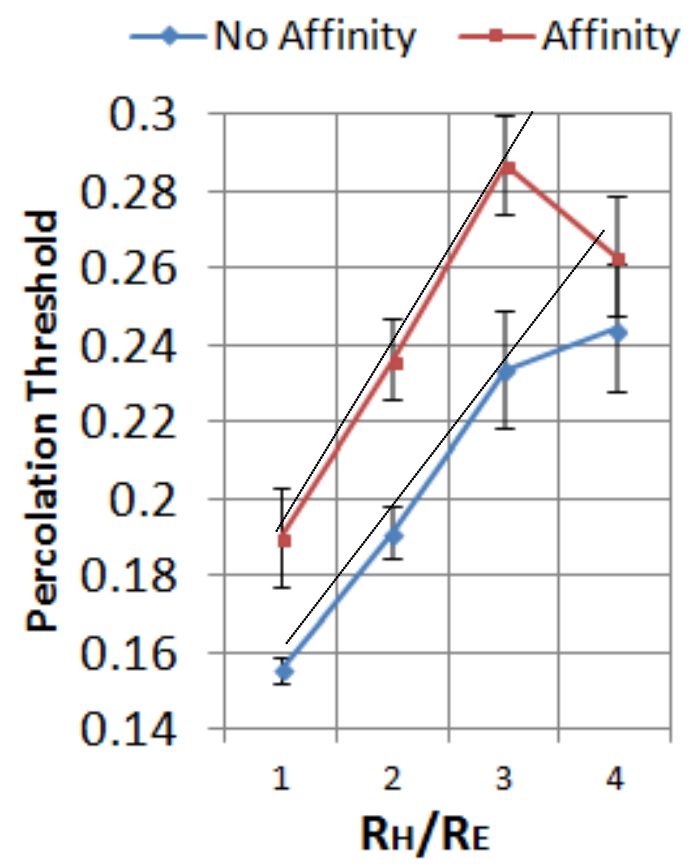

Fig. 2. Percolation threshold $\left(\boldsymbol{\rho}_{c}\right)$ extended to $R_{H} / R_{E}=4$ showing a dip in trend 
An explanation for this deviation from linearity is shown in Fig. 3. As the $\mathbf{E}$ phase (blue) particle size decreases relative to $\mathbf{H}$, the $\mathbf{E}$ particles move in between the $\mathbf{H}$ particles, pushing the $\mathbf{H}$ particles apart. However, as the $\mathbf{E}$ particles continue to shrink relative to the $\mathbf{H}$, the $\mathbf{E}$ can no longer push the $\mathbf{H}$ phase apart. This implies a maximum relative size above which the percolation threshold between $\mathbf{H}$ particles will decrease rather than increase because the $\mathbf{E}$ particles may no longer separate the $\mathbf{H}$ particles because the $\mathbf{E}$ particles simply are too small and they are pushed into interstitial space (space remaining if $\mathbf{H}$ particles were touching) where they cannot as readily separate $\mathbf{H}$ particles. It is noted, however, in this simulation the Leonard-Jones pairs-wise particle potential used in this study does not allow for a lateral force or frictional component. So, in practice under experimental conditions we would not expect the $\mathbf{E}$ particles to be as easily pushed out of the way because the $\mathbf{E}$ particles would experience friction.

It is also apparent in Fig. 2 that this "dip” in the linear $\boldsymbol{\rho}_{\mathbf{c}}$ vs. size trend is larger when there is an affinity between $\mathbf{H}$ and $\mathbf{E}$ particles. This is because when two $\mathbf{H}$ particles are attracted to the same $\mathbf{E}$ particles they are also attracted to each other, and if attracted to each other they will become closer together thus reducing the percolation threshold $\left(\boldsymbol{\rho}_{\mathrm{c}}\right)$.
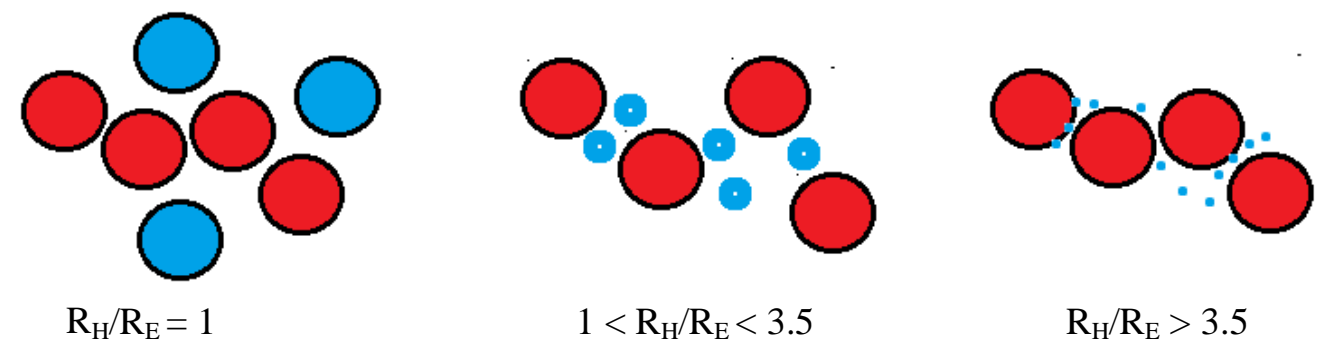

Fig. 3. Illustration of particle size effect $(\operatorname{Red}=\mathbf{H}$, Blue $=\mathbf{E})$ 


\subsection{Analysis}

For $\mathrm{R}_{\mathrm{H}} / \mathrm{R}_{\mathrm{E}}=4$, Fig. 4 shows two radial distribution functions after the gelation stage (GEL) at a volume fraction of 30\% for a case of no particle affinity (Noa) (a) on the left, and with H-E particle affinity (b) on the right. In theory, if all particles were only in direct contact with their nearest neighbors then the center-to-center distance for the $\mathbf{H}$ particles would be $\mathrm{D}=1.12246 \sigma$, the $\mathbf{H}$-E center-to-center distance would be $\mathrm{D} / 2+\mathrm{D} / 8=(5 / 8) \times 1.12246=0.70154 \sigma$, and the center-to-center distance for $\mathbf{E}$ particles would be $\mathrm{D} / 5=0.22449 \sigma$. It can be seen in Fig 4(a) (Noa) that the $\mathbf{H}-\mathbf{H}$ center-to-center distance is $1.31 \sigma$, the H-E distance is $0.74 \sigma$, and the E-E distance is $0.34 \sigma$. In Fig. 4(b) when H-E affinity is applied, the corresponding $\mathbf{H}-\mathbf{H}$ center-to center distance is $1.31 \sigma$, the $\mathbf{H - E}$ distance is $0.74 \sigma$, and the $\mathbf{E}$-E distance is 0.31б. Therefore, there is no appreciable shift in peaks abscissa values when H-E affinity is invoked. The primary peaks for $\mathrm{R}_{\mathrm{H}} / \mathrm{R}_{\mathrm{E}}=2$ and 3 also do not move when $\mathbf{H}-\mathbf{E}$ affinity is applied. However, in both Fig. 5 and Fig. 6, it is noticed that the $\mathbf{H}-\mathbf{H}$ primary peak loses some area to the $\mathbf{H}-\mathbf{H}$ secondary peak which grows, but the primary peak remains larger. But in Fig. 6, the primary H-H peak looses sufficient area to the $\mathbf{H}-\mathbf{H}$ secondary peak such that the secondary peak is then larger than the primary. This reinforces the explanation per Fig. 3 how it is that the percolation threshold $\left(\boldsymbol{\rho}_{c}\right)$ is largest at $R_{H} / R_{E}=3$, which is that there must be a combination of steric effects (geometry) and particle forces that results in an optimal relative particle size that maximizes $\boldsymbol{\rho}_{\mathbf{c}}$.
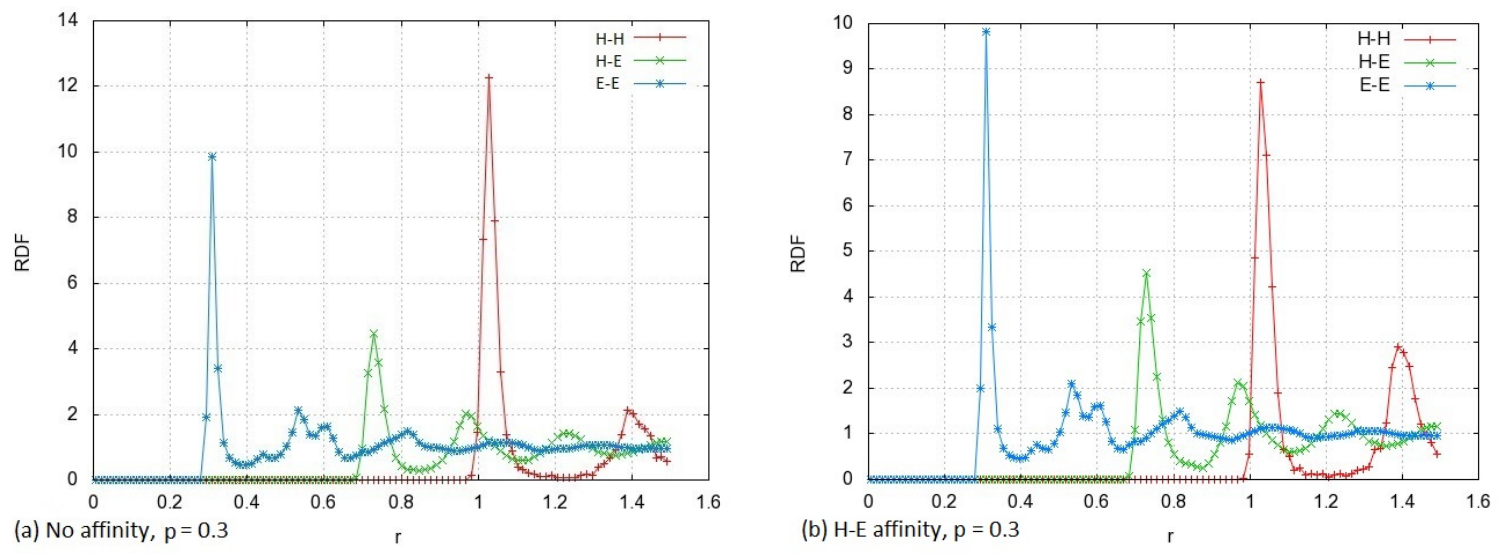

Fig. 4. Radial Distribution Functions for $\mathbf{H}$ and $\mathbf{E}$ phases, $p=0.3, R_{H} / R_{E}=4$ 

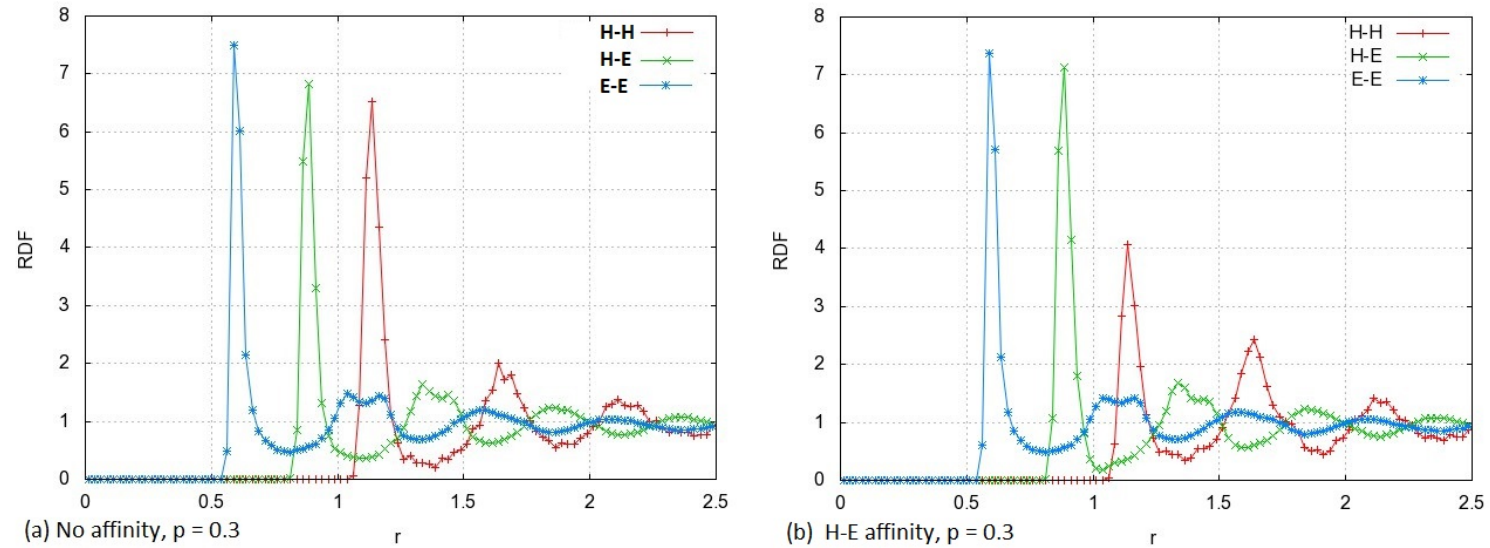

Fig. 5. Radial Distribution Functions for $\mathbf{H}$ and $\mathbf{E}$ phases, $p=0.3, R_{H} / R_{E}=2$
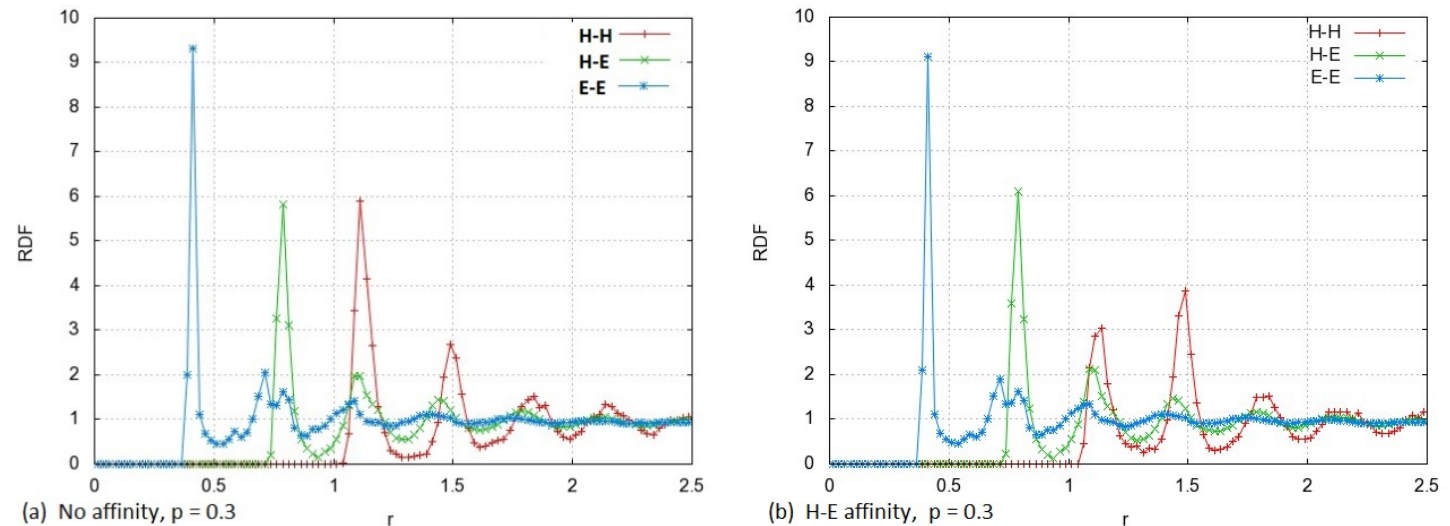

Fig. 6. Radial Distributions Functions for $\mathbf{H}$ and $\mathbf{E}$ phases, $p=0.3, R_{H} / R_{E}=3$

\subsection{Supporting analysis and results for comparison of thermodynamics to Chapter 2}

Fig. 7 shows the potential and kinetic energy curves for the case no particle affinity where particle size is $R_{H} / R_{E}=4$, and the volume fraction $\left[V_{H} /\left(V_{H}+V_{E}\right)\right]=0.3$. The general character of the energy curves for $R_{H} / R_{E}=4$ are the same as those reported for $R_{H} / R_{E}=1,2,3$ in chapter 2 . In the mixing stage, Fig 7 (left), periodic boundary conditions are on and the packing fraction is low where $f=0.5236$. This allows sufficient room for mixing and periodic boundary conditions particle segregation. For the MIX stage, the temperature is ramped from 0.01 to $0.2 \varepsilon / \mathrm{k}_{\mathrm{B}}$ ( $\mathrm{k}_{\mathrm{B}}$ is the Boltzmann constant) for the first $1,000,000$ time steps, then the temperature is fixed at a constant value of $0.2 \varepsilon / \mathrm{k}_{\mathrm{B}}$ for the remaining nine million time steps. The initial temperature ramp from 0.01 to $0.2 \varepsilon / \mathrm{k}_{\mathrm{B}}$ was done to equilibrate the 
particles from their simple cubic lattice starting positions by allowing the particles to react only to forces from other particles before kinetic energy is introduced. After one million time steps the particles are mixed at a relatively hot temperature of $0.2 \varepsilon / \mathrm{k}_{\mathrm{B}}$ to ensure complete mixing. In the equilibration stage (EQU) Fig. 7 (middle), the potential energy curve slowly increases indicating the particles are equilibrating as the stresses between particles are relaxed. This occurs because the packing fraction while increased to $f=0.755 \times(20 / 21)^{3}=0.65$ is still below the jamming packing fraction of $f=0.755$ [1] [2] in the case of $R_{H} / R_{E}=4$. For the gelation stage (GEL) Fig. 8 (right), the potential energy is becoming more negative indication gelation or glass transition to a solid state is occurring. It may be noticed that the values of the potential energy for $R_{H} / R_{E}=4$ is approximately $25 \%$ larger than equivalent values for $R_{H} / R_{E}$ $=3$ and approximately 10 times larger than equivalent values reported for $R_{H} / R_{E}=2$ in chapter 2 . This is because the distance between the particle centers is smaller as $\mathrm{R}_{\mathrm{H}} / \mathrm{R}_{\mathrm{E}}$ becomes larger, and while the Leonard-Jones sigma $(\sigma)$ values was adjusted for each different particle size, the energy or Leonard-Jones well depth was left constant at $10 \varepsilon$ during the GEL stage.

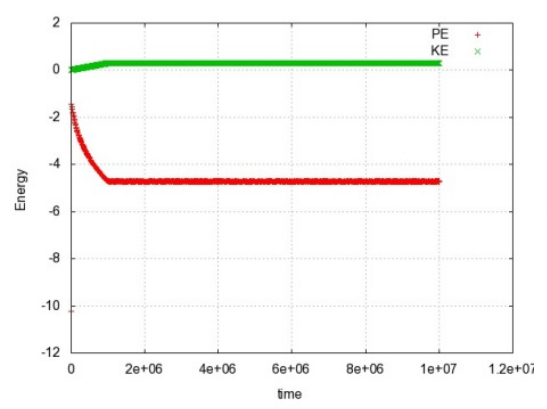

MIX

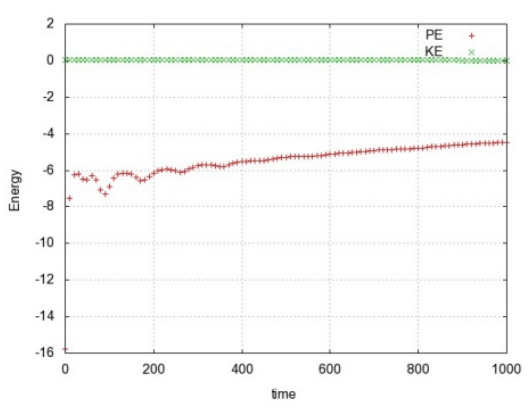

EQU

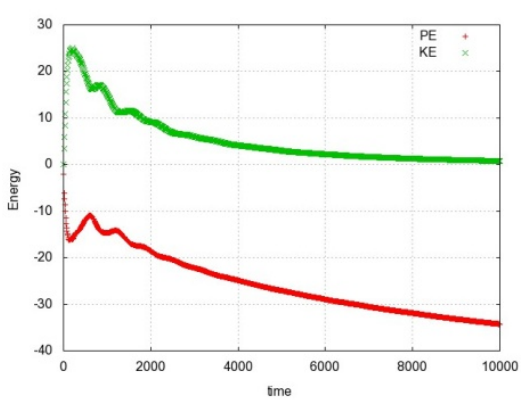

GEL

Fig. 7. Kinetic and Potential Energy during all three stages of mixing, equilibration and gelation for a sample case $\mathrm{R}_{\mathrm{H}} / \mathrm{R}_{\mathrm{E}}=4$, no affinity (Noa), $\mathrm{p}=$ volume ratio $=0.3$

Fig. 8 shows a case of the spatial coordinates for a case with no particle affinity for $p=0.3$ after the GEL stage for $R_{H} / R_{E}=4$ were it can be seen the particles are well mixed. As in chapter 2 for $R H / R E=1$, 2, 3 complete inter-phase mixing is required to maximize the percolation threshold and avoid particle segregation [3] [4] [5]. 

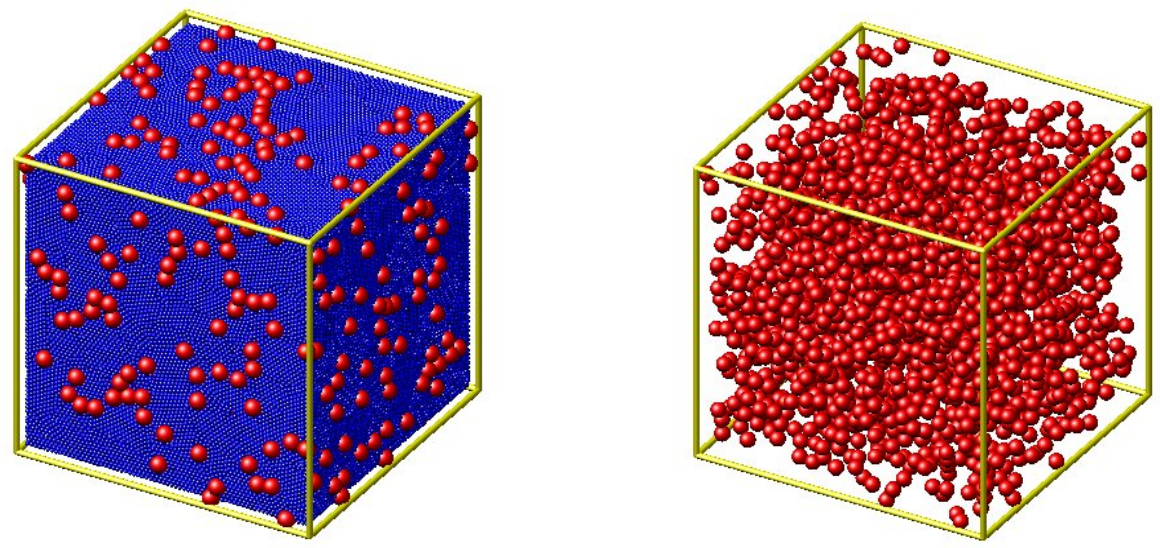

Fig. 8. Image of hard-shell particle simulation after GEL stage with no particle affinity and volume ratio $\mathrm{p}=0.3$ Left: Both $\mathbf{H}$ (red) and $\mathbf{E}$ (blue) articles. Right: $\mathbf{H}$ only showing particles well mixed

Shown in Fig. 9, for all simulations is the average local volume fraction $\left(\mathbf{p}_{\mathbf{a}}\right)$ which is the volume of $\mathbf{H}$ phase per volume of both phases in a small sphere of radius 2D about every $\mathbf{H}$ particle. The $\mathbf{p}_{\mathbf{a}}$ is close to the global volume fraction $\left(\mathbf{p}_{\mathbf{c}}\right)$ corresponding to the smallest $\boldsymbol{\rho}_{\mathbf{c}}$ where $\mathrm{D}=1.12246 \sigma$. As can be seen in Fig. 9 that $\mathbf{p}_{\mathbf{a}}$ is close to $\mathbf{p}_{\mathbf{c}}$ (lowest global volume fraction where percolation occurs) for all cases. Thus all mixtures are maintained sufficiently uniform after the GEL stage to maximize $\mathbf{H}-\mathbf{H}$ electrical percolation.

\begin{tabular}{c|c|l|l} 
Affinity & $\mathrm{R}_{\mathrm{H}} / \mathrm{R}_{\mathrm{E}}$ & $\mathrm{p}_{\mathrm{a}}$ & $\mathrm{p}_{\mathrm{c}}$ \\
\hline $\mathrm{N}$ & 1 & $0.2429(0.0247)$ & 0.2433 \\
\hline $\mathrm{N}$ & 2 & $0.2780(0.0136)$ & 0.2833 \\
\hline $\mathrm{N}$ & $\mathbf{3}$ & $0.3213(0.0127)$ & 0.320 \\
\hline $\mathrm{N}$ & 4 & $0.3121(0.0253)$ & 0.3267 \\
\hline $\mathrm{N}$ & AVG & 0.2965 & 0.2933 \\
\hline $\mathrm{A}$ & 1 & $0.2996(0.0065)$ & 0.2967 \\
\hline $\mathrm{A}$ & 2 & $0.3375(0.00987)$ & 0.350 \\
\hline $\mathrm{A}$ & $\mathbf{3}$ & $0.3984(0.0252)$ & 0.3933 \\
\hline $\mathrm{A}$ & 4 & $0.3600(0.0054)$ & 0.3567 \\
\hline $\mathrm{A}$ & AVG & 0.3516 & 0.3492
\end{tabular}

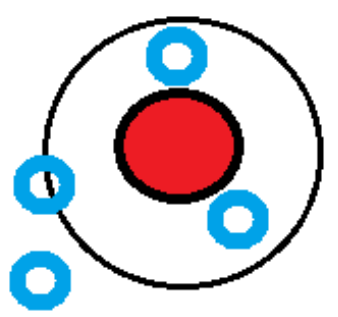

Fig. 9. Average local volume fraction ( $\left.\mathbf{p}_{\mathbf{a}}\right)$ (std. dev. three trials) is statistically equal to the global volume fraction $\mathbf{p}_{\mathbf{c}}=\mathrm{V}_{\mathrm{H}} / \mathrm{V}_{\mathrm{H}}+\mathrm{V}_{\mathrm{E}}$ 
6.0 Results and analysis for mixture of all particle size ratios in single simulation

Fig. 10 show a case after the GEL stage, for no affinity, and volume fraction $\mathrm{p}=0.3$ where all particles sizes are mixed together in equal amounts. That is for a given volume fraction of $\mathbf{H}$, the remaining $\mathbf{E}$ particles are divided into 4 groups for $\mathrm{R}_{\mathrm{H}} / \mathrm{R}_{\mathrm{E}}=1$, 2, 3, 4, and each group constitutes $25 \%$ of the particles out of the $\mathbf{E}$ phase. Fig. 11 shows the corresponding radial distribution function and kinetic and potential energy curves for the simulation results shown in Fig. 10. The radial distribution functions and energy curves in Fig. 11 are similar to others for individual particle size ratios $\left(\mathrm{R}_{\mathrm{H}} / \mathrm{R}_{\mathrm{E}}\right)$ in chapter 2 , and verify the expected inter-particle distances and the arrest of particle motion as expected during the gelation stage. The percolation threshold $\boldsymbol{\rho}_{\mathbf{c}}$ is plotted versus the packing fraction rather than the size ratio $\left(R_{H} / R_{E}\right)$ because in this case $R_{H} / R_{E}$ is indeterminate. However, per Fig. 11, the percolation threshold ( $\boldsymbol{\rho}_{\mathbf{c}}$ ) is lower than would be expected based on comparison to the other cases for $\mathrm{R}_{\mathrm{H}} / \mathrm{R}_{\mathrm{E}}=1,2,3$. It is hypothesized this is because the solutions for mixture of all particles form a superposition of solutions for each individual case. Therefore, the percolation threshold can be any value previous reported for single particle ratio cases. In this case, mixing all particles sizes together results in a percolation threshold landing somewhere between the $\mathrm{R}_{\mathrm{H}} / \mathrm{R}_{\mathrm{E}}=1$ to 2 . It is concluded that allowing a mixture of all particles sizes diminishes the effects of reduced relative particles size and inter-phase $\mathbf{H}-\mathbf{E}$ particle affinity. 


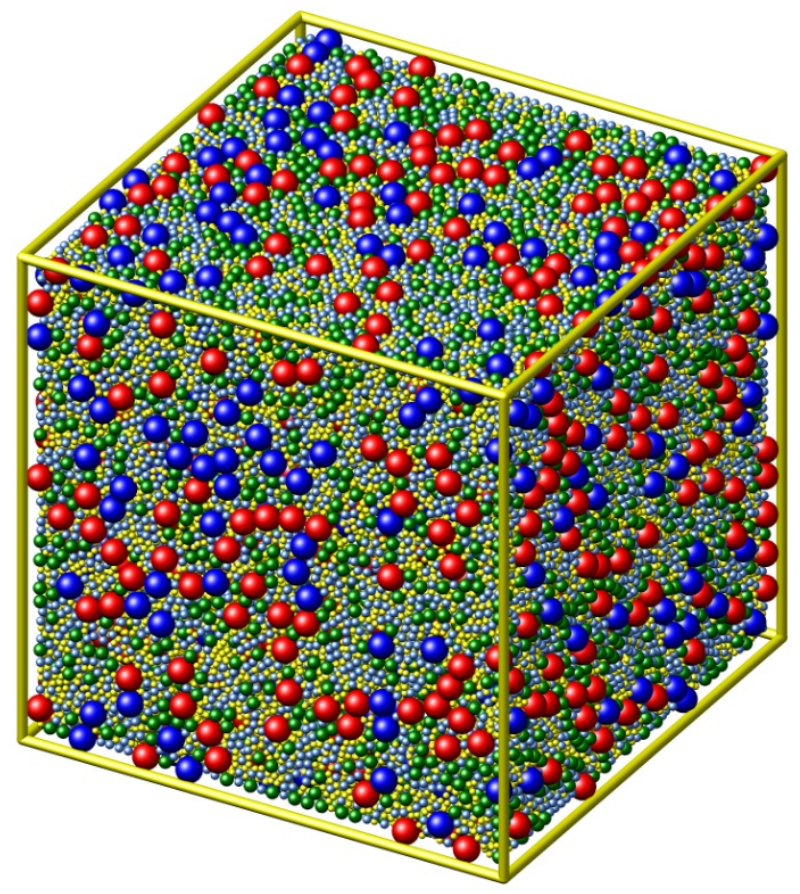

Fig. 10. Post GEL stage results for all sizes $\mathrm{RH} / \mathrm{RE}=1,2,3,4$ each in equal number perportions of $25 \%$ of all E phase particles
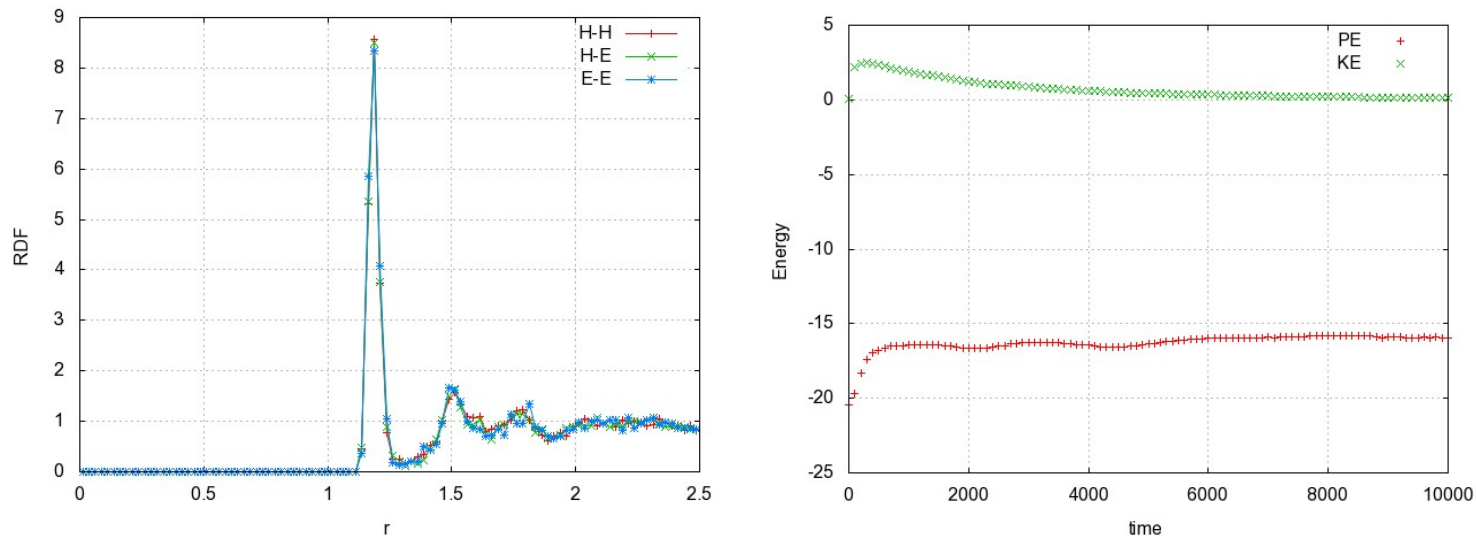

Fig. 11. (left) radial distribution function and (right) kinetic and potential energy curves corresponding to the GEL stage per Fig. 10. 


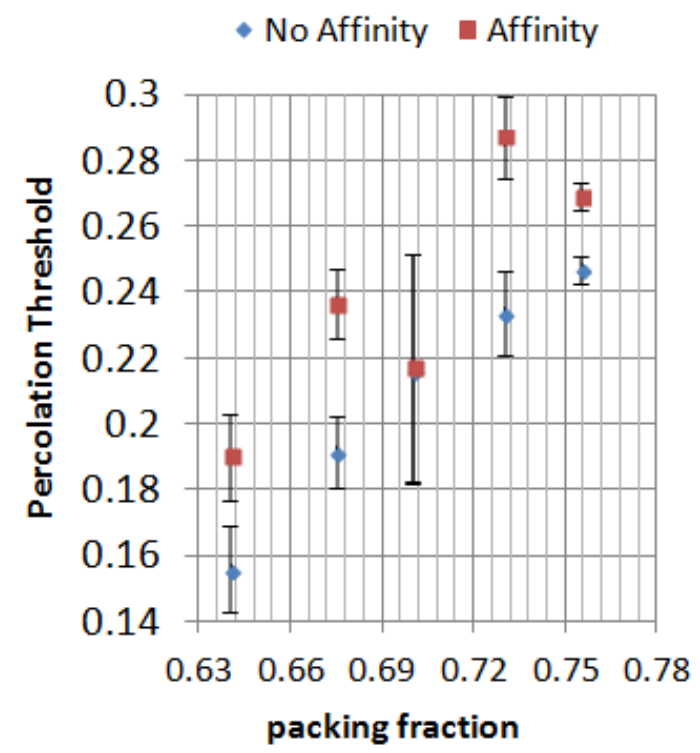

Fig. 12. Percolation threshold $(\rho c)$ versus the packing fraction

\section{References}

1. A. Donev, S. Torquato, F. H. Stillinger, R. Connley (2003) Jamming in hard sphere and disk packings. J. Appl Phys. 95(3):989-997

2. A.R. Kansal, S. Torquato, F.H. Stillinger (2002) Computer generation of dense polydisperse sphere packings. J. Chem. Phys. 117(18):8212-8218

3. S.W. Meier, R.M. Lueptow, J.M.Ottino (2007) A dynamical systems approach to mixing and segregation of granular materials in tumblers. Advances in Physics 56(5):757-827

4. J.M. Ottino, D.V. Khakhar (2000) Mixing and segregation of granular materials. Annu. Ref. Fluid Mech. 32:55-91

5. K. van der Vaart, P. Gajjar, G. Epely-Chauvin, N. Andreini, J.M.N.T. Gray, C. Ancey (2015) Underlying asymmetry with in particle size segregation. Phys. Rev. Lett. 114(238001):1-5 


\section{Chapter 5}

\section{Conclusions and future work}

\subsection{Conclusions}

1.1 Conclusions based on particulate hard shell only simulations

Based upon the results from chapters 2 and 4, as the piezoelectric $\mathbf{E}$ particle size decreases relative to the magnetostrictive $\mathbf{H}$ particles, the inter-phase $\mathbf{H}-\mathbf{H}$ electrical percolation threshold increases because the $\mathbf{E}$ particles are interposing between the $\mathbf{H}$ particles. Further, particle affinity and short range attraction between the $\mathbf{H}$ and $\mathbf{E}$ particles facilitates a mechanism whereby the larger $\mathbf{H}$ particles are covered or painted with smaller $\mathbf{E}$ particles, so that particle affinity also increases the percolation threshold. However, if the smaller $\mathbf{E}$ particles become too small, their ability to interpose between and separate the $\mathbf{H}$ particles is diminished because rather the $\mathrm{E}$ particles get pushed into interstitial space (space between spheres in contact). Therefore, it is concluded that to maximize the percolation threshold the ratio of the radii of the larger to smaller particles size should be $R_{H} / R_{E}=3.5$. In addition, the spread of particles size about this optimal size of 3.5 should be as narrow as possible.

\subsection{Conclusions regarding particle deformation}

Based upon the results from chapter 3, the shape of the magnetostrictive $\mathbf{H}$ phase that minimizes percolation thus maximizing the percolation threshold is spherical. Even a modest amount of deformation such as demonstrated when the radius of gyration grew from $0.435 \sigma$ to $0.592 \sigma$ for deformed $\mathbf{H}$ particles in chapter 3 is sufficient to decrease the percolation threshold by a factor of approximately 2, and in practice experimentally probably by a factor of 5 or more. However, deformation of the piezoelectric $\mathbf{E}$ phase around rigid spherical $\mathbf{H}$ particles, on the other hand, will increase the percolation 
threshold. Therefore, to maximize the magnetoelectric (ME) effect an optimal choice of relative particle sizes and particles affinities is required.

\subsection{General recommendations for applications of the results of this study}

Based upon the results of this study, in practice it is implied that for a magnetostrictive $\mathbf{H}$ particles of size $100 \mathrm{~nm}$, a monodisperse distribution of $\mathbf{E}$ particles with $\mathbf{E}$ particles (3.5) ${ }^{-1}$ the size of the $\mathbf{H}$ particles is optimal for maximizing the ME effect. On the other hand, maximization of mechanical contact between the $\mathbf{E}$ and $\mathbf{H}$ phases occurs the larger the particle size ratio $R_{H} / R_{E}$ is. In addition, across all cases of particles size, particle affinity between the $\mathbf{H}$ and $\mathbf{E}$ phases universally pushes the magnetostrictive percolation threshold up. Therefore, the following are suggested to minimize the conductive percolation or conductivity across the magnetostrictive $\mathbf{H}$ phase of a magnetoelectric composite while maximizing the magnetoelectric (ME) effect.

1. Mix the particles completely during the pre-sintering or mixing stage sufficiently to avoid phase particle separation that may occur as a result of particle dynamics and rheology.

2. Use an asymmetric relative size distribution of particles sizes with a sharp drop off in relative particles sizes $R_{H} / R_{E}<3.5$, but with a longer tail on the right side of the particle distribution for particles $\mathrm{R}_{\mathrm{H}} / \mathrm{R}_{\mathrm{E}}>3.5$ to maximize magnetoelectro mechanical contact.

3. Perform sintering maintaining the temperature below the melting points of both the magnetostrictive and piezoelectric precursor phases, but if possible with an $\mathbf{H}$ precursor melting temperature above the $\mathbf{E}$ phase melting point.

4. Maximize inter-phase $\mathbf{H}-\mathbf{E}$ affinity whenever possible, perhaps with a binding agent during sintering or a third phase that would act as an adhesive but not substantially diminish inter-phase mechanical contact. 


\subsection{Future work}

The results of these simulations were based upon the Leonard-Jones potential energy function which only has a radial component and is frictionless. Therefore, an improvement to the methods employed here would be to use a granular potential [1] [2] where both friction and lateral forces between all particles as well as angular moment of all particles would be incorporated into the particulate simulation model in LAMMPS [3-6].

In chapter 2 it was shown that up to a certain critical particle size ratio $\mathrm{R}_{\mathrm{H}} / \mathrm{R}_{\mathrm{E}}=3.5$, the percolation threshold of the larger particulate phase increases linearly, but after this critical size ratio, the linear trends dips. The nature of this dip in the trend and particular critical value at which it occurs bears further investigation. With a granular potential for incorporating friction into the simulations, using incremental steps of $R_{H} / R_{E}$ values of 3, 3.2 up to 5.0 additional simulations could be conducted to flesh out the details of how and why the percolation threshold falls. Granular simulations could be developed by varying both the particle affinity and the run times and temperatures which should be mapped to corresponding experimental measurements strengthening the methods used in this study's ability to predict the outcomes of practical experiments.

Quantum tunneling [7], the ability of electrons to tunnel through classically forbidden regions and the wave nature of conductivity was assumed in this study to be of fixed effect with an increase in conductivity range of $5 \mathrm{~nm}$ between two insulated particles. Although based on other results [8] [9] [10], this is a valid but undeveloped assumption. In actuality, electron transport depends on many factors like the quantum orbitals of constituent atoms and the capacitive dielectric properties between molecules, and to this end, Boltzmann particle transport theory [11] and the phenomena of quantum electron hopping [8] [12] [13] could be incorporated to adapt this work for the effects of quantum mechanics.

Per [2] [14], the phenomena of dynamic segregation as a result of particle flow and rheology presents a problem to the goal of maximizing the magnetostrictive percolation threshold. Therefore, additional 
studies should be conducted to find the optimal experimental technique needed to maximize the isolation of the $\mathbf{H}$ phase particles during the "pre-sintering" phase of $\mathbf{M E}$ composite preparation.

Finally, the processes of sintering (increasing particle contact and material density) such as applying pressure and temperature in solid state methods, or use of binding agents in liquid sintering, or the use of electricity and or microwaves are varied and extensive [15] [16]. Each technique as emphasizes different factors such as the interplay between coarse graining and densification [16]. Additional studies should be conducted to explore the applications of varied and clever sintering methods to the problem of optimizing the magnetoelectric effect.

\section{References}

1. J.M. Ottino, D.V. Khakhar (2000) Mixing and segregation of granular materials. Annu. Rev. Fluid Mech. 32:55-91

2. S.W. Meier, R.M. Lueptow, J.M. Ottino (2007) A dynamical systems approach to mixing and segregation of granular materials in tumblers. Adv. in Phys. 56(5):757-827

3. M. Prateek (2012) DEM simulation of flow of granular particles on an inclined plane in LIGGGHTS. https://www.researchgate.net/publication/236586147

4. V. Vedachalam (2011) Discrete element modeling of granular snow particles using LIGGGHTS, MS Thesis, Edinburgh Parallel Computing Center, UK.

5. C. Kloss, C. Goniva (2010) Granular simulations in LAMMPS, Lammps workshop Johannes Kepler University, Linz.

6. I.J.T Padding (2013) Particle-based simulations (6KM59) http://padding.awardspace.com/Particle-based_Simulations_Padding-small.pdf

7. D. Carrera (2007) Quantum tunneling in chemical reactions. https://www.princeton.edu/chemistry/macmillan/group-meetings/DEC_tunneling.pdf

8. I.S. Beloborodov, A.V. Lopatin, V.M. Vinokur (2005) Coulomb effects and hopping transport in granular materials. Phys. Rev. B 72:125121 
9. S. Kadkhodazadeh, J.B. Wagner, H. Kneipp, K. Kneipp (2013) Coexistance of classical and quantum plasmonics in large plasmonic structure with subnanometer gaps. Appl. Phys. Lett. 103:083103

10. J. Li, J-J. Kim (2007) Percolation threshold of conducting polymer composites containing 3D randomly distributed graphite nanoplatelets. Composites Science and Technology 67:2114-2120

11. R.R. Nourgaliev, T.N. Dihn, T.G. Theofanous, D. Joseph (2003) The lattice Boltzmann equation method: theoretical interpretation, numerics and implications. Int. J. Multiphase Flow 29:117-169

12. V. Ambegaokar, B.I. Halperin, J.S. Langer (1971) Hopping conductivity in disordered systems. Phys. Rev. B 4(8):2612-2620

13. Z.Shuai, H. Geng, W. Xu, Y. Liao, J-M.Andre (2014) From charge transport parameters to chare mobility in organic semiconductors through multiscale simulation. Chem. Soc. Rev. 43:26622679

14. D.C. Rapaport (2014) Molecular dynamics simulation: a tool for exploring and discovery using simple models. J. Phys: Condens. Matt. 26:503104

15. J.K. Mackenzie, R. Shuttleworth (1949) A phenomenological theory of sintering. Proc. Phys. Soc. LXII, 12-B.

16. L.C. DeJonghe, M.N. Rahaman (2003) Sintering of Ceramics Chapter 4, Handbook of Advanced Ceramics, S. Somiya (editors), Elsevier Inc. 
Appendix A. Programs used in hard-shell modeling (Chapter 2 [1])

The following list is one example of the order in which the programs in this appendix were run to produce the output in [Bar17]. All programs in this appendix were run on a model ASUS 2.53 GHZ running 64 bit Windows 7 Home Premium 2009. LAMMPS @ was run on the West Virginia University High Performance Cluster running a UNIX operating system.

$<\mathbf{p}>$ is inline parameter for volume fraction.

$<\mathbf{R}_{\mathbf{m}}>$ is inline parameter for maximum center to center distance or range wherein particles are connected.

$\underline{\text { Run program }} \quad \underline{\text { Explanation }} \quad \underline{\text { Page }}$

1. pmpe4.c $<\mathrm{p}>\quad$ Produce set of input coordinates for $\mathrm{R}_{\mathrm{H}} / \mathrm{R}_{\mathrm{E}}=4$.

$$
\mathrm{p}=\text { number fraction }=\mathrm{N}_{\mathrm{H}} /\left(\mathrm{N}_{\mathrm{H}}+\mathrm{N}_{\mathrm{E}}\right)
$$

2. lammps $<$ input_MIX.txt $\quad$ Run Lammps. See appendix C for MIX scripts

3. cvt_dmp_inp2.pl Convert output Lammps dump file to input data file.

4. lammps < input_EQU.txt Run Lammps. See appendix C for EQU script

5. cvt_dmp_inp3.pl Convert output dump file to input data file.

6. lammps < GEL.txt Run Lammps. See appendix C for GEL script

7. filt_coords_PM-2.pl Strip unnecessary information from dump file. 95

8. filt_coords_PE-2.pl Strip unnecessary information from dump file. 96

9. filt_coords_PM-R.pl Strip unnecessary information from dump file. 97

10. dist3.c $<\mathrm{Rm}>\quad$ Create file of bond pairs. $\mathrm{Rm}=$ connection radius. $\mathrm{Rm}=$ maximum range between centers of particles wherein particles are connected. 98

11. zap2.c $<$ Rm $>\quad$ Recurse through bond pairs and evaluate percolation. $\quad 99$ 
12. CNHH.c $<$ Rm $>\quad$ Calculate c.n. for all $\mathrm{H}$ particles with respect to other $\mathrm{H}$ particles. 102

13. pa64.c Calculate local packing fraction.

103

\section{References}

1. E.J. Barbero, A.J. Bedard (2017) Electrical percolation threshold of magnetostrictive inclusions in a piezoelectric matrix composite as a function of relative particle size. Computational Particle Mechanics (printed online 25 July 2017 ISSN 2196-4378)

2. D. Stauffer, A. Aharmoney (1994) Introduction to Percolation Theory. $2^{\text {nd }}$ Ed. Taylor and Francis, London UK.

3. R.L. Kruse (1989) Programming with data structures, Prentice Hall, Chap. 17

4. S.J.Plimpton (1995) LAMMPS (c) Fast parallel algorithms for short-range molecular dynamics. J. Comp. Phys. 117:1-19 
Programs used in Chapter 2

$/ *$

pmpe4.c 14 Dec 16 J.Bedard

Purpose: Produce random random coordinates when RH/RE $=4 / 1$

Usage: pmpe4 <vol_fract\%>

*/

\# include <stdio.h>

\# include <stdlib.h>

\# include <math.h>

\# include <string.h>

int Box1(float $x$, float $y$, float $z$, float $d x$, float dy, float $d z$, int ndx, int typ, int $\left.Q, F I L E{ }^{*} f p 1\right)$;

int Box2(float $x$, float $y$, float $z$, float $d x$, float dy, float $d z$, int ndx, int typ, int $\left.Q, F I L E{ }^{*} f p 1\right)$;

int Box4(float $x$, float $y$, float $z$, float $d x$, float dy, float $d z$, int ndx, int typ, int $\left.Q, F I L E{ }^{*} f p 1\right)$;

int main(int argc, char *argv[])\{

FILE *fp1; fp1=fopen("data1.dat", "w");

int $i, j, k$, atoms, chg, sx, sy, sz, ndx, typ, Q, PM;

int atoms2;

float sdx, sdy, sdz, dx, dy, dz, x,y, z, prc, vf;

char str[20];

float rnd;

srand(( unsigned)time(NULL));

strcpy(str, argv[1]); $\operatorname{prc}=\operatorname{atof}(s t r) ;$

$I^{*} \operatorname{strcpy}(\operatorname{str}, \operatorname{argv}[2]) ; \quad$ offset $=\operatorname{atoi}(\mathrm{str}) ; * /$

/* CONSTANTS \& <<COMPILE */

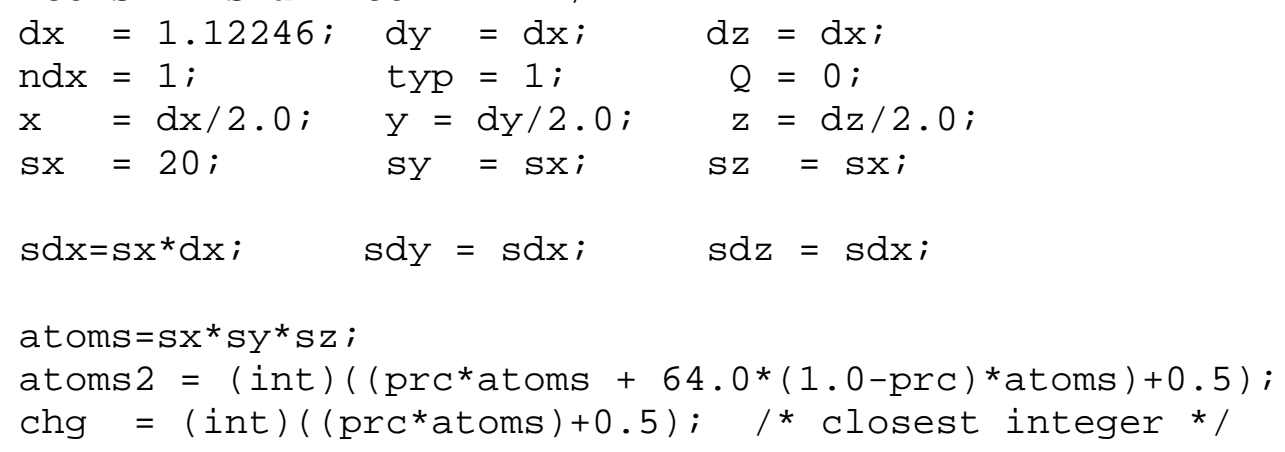

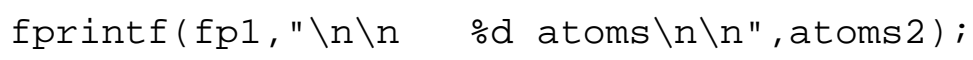




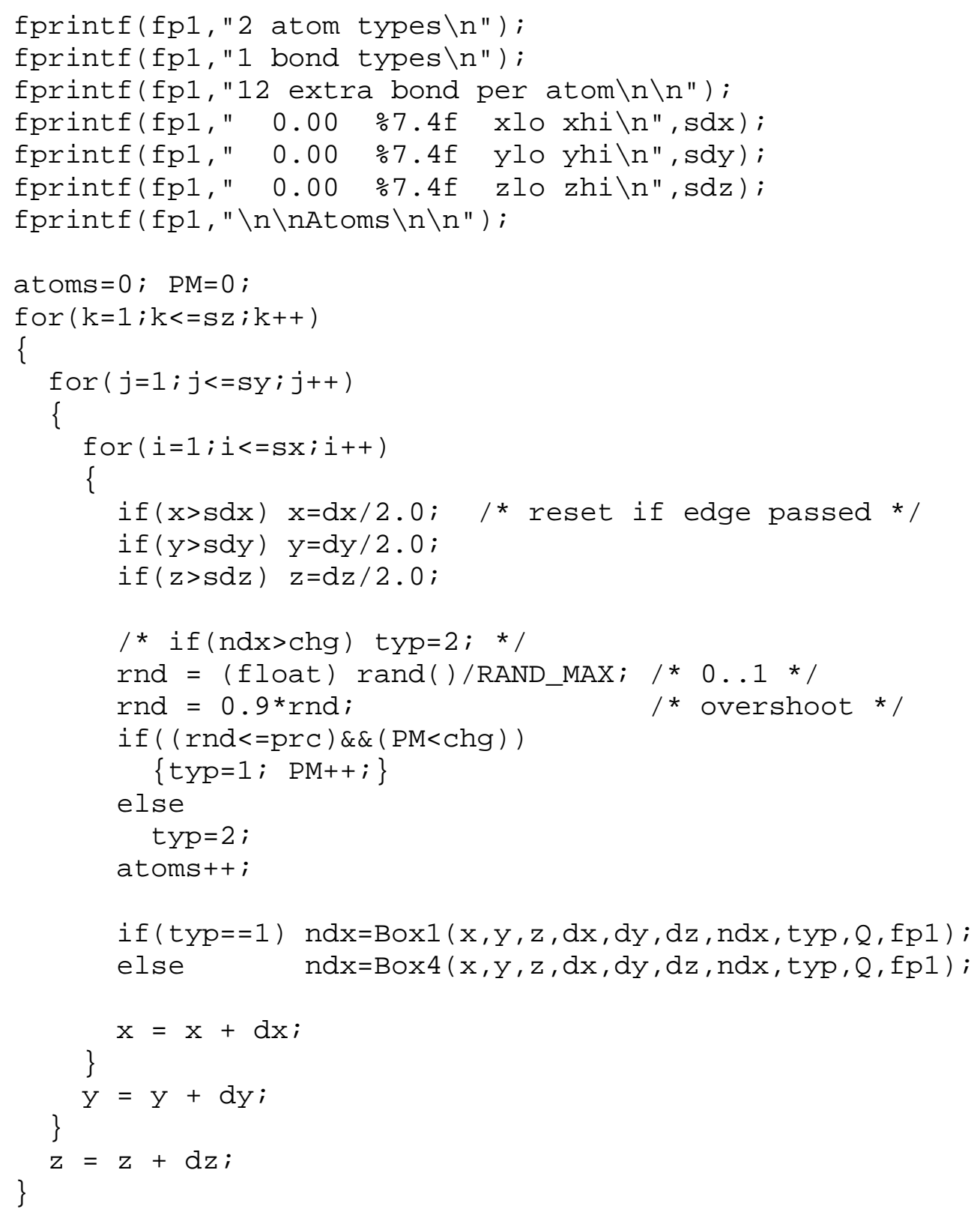


return(ndx);

\}

int Box2(float $x$, float $y$, float $z$, float $d x$, float dy, float $d z$, int ndx, int typ, int $Q, F I L E ~ * f p 1)$

\{

int $i, j, k$;

float $x 2, y 2, z 2$;

$\mathrm{x} 2=x ; y 2=y ; \quad z 2=z ; \quad /$ * always adjust from initial xyz */

$\mathrm{dx}=\mathrm{dx} / 2.0 ; \quad \mathrm{dy}=\mathrm{dy} / 2.0 ; \mathrm{dz}=\mathrm{dz} / 2.0$;

$x=x 2+d x ; y=y 2+d y ; z=z 2+d z ;$

fprintf(fp1, "\%d \%d \%d \%d \%f \%f \%f\n", ndx, ndx, typ, $Q, x, y, z)$;

$n d x++; \quad x=x 2+d x ; y=y 2+d y ; z=z 2-d z$;

fprintf(fp1, "\%d \%d \%d \%d \%f \%f \%f\n", ndx, ndx, typ, $Q, x, y, z)$;

$n d x++; \quad x=x 2+d x ; y=y 2-d y ; z=z 2+d z$;

fprintf(fp1, "\%d \%d \%d \%d \%f \%f \%f\n", ndx, ndx, typ, $Q, x, y, z)$;

$n d x++; \quad x=x 2+d x ; y=y 2-d y ; z=z 2-d z$;

fprintf(fp1, "\%d \%d \%d \%d \%f \%f \%f\n", ndx, ndx, typ, $Q, x, y, z)$;

$n d x++; \quad x=x 2-d x ; y=y 2+d y ; z=z 2+d z ;$

fprintf(fp1, "\%d \%d \%d \%d \%f \%f \%f\n", ndx, ndx, typ, $Q, x, y, z)$;

$n d x++; \quad x=x 2-d x ; y=y 2+d y ; z=z 2-d z$;

fprintf(fp1, "\%d \%d \%d \%d \%f \%f \%f\n", ndx, ndx, typ, $Q, x, y, z)$;

$n d x++; \quad x=x 2-d x ; y=y 2-d y ; z=z 2+d z$;

fprintf(fp1, "\%d \%d \%d \%d \%f \%f \%f\n", ndx, ndx, typ, $Q, x, y, z)$;

$n d x++; \quad x=x 2-d x ; y=y 2-d y ; z=z 2-d z$;

fprintf(fp1, "\%d \%d \%d \%d \%f \%f \%f\n", ndx, ndx, typ, $Q, x, y, z)$; $\mathrm{ndx}++$;

return $(\mathrm{ndx})$;

\}

int Box4(float $x$, float $y$, float $z$, float $d x$, float dy, float $d z$, int ndx, int typ, int $\left.Q, F I L E{ }^{*} f p 1\right)$

\{

int $i, j, k$;

float $\mathrm{x} 2, \mathrm{y} 2, \mathrm{z} 2$;

$\mathrm{x} 2=\mathrm{x} ; \mathrm{y} 2=\mathrm{y} ; \mathrm{z2}=\mathrm{z} ; \quad /$ * always adjust from initial $\mathrm{xyz}$ */

$\mathrm{dx}=\mathrm{dx} / 4.0 ; \mathrm{dy}=\mathrm{dy} / 4.0 ; \mathrm{dz}=\mathrm{dz} / 4.0$;

$\mathrm{x}=\mathrm{x} 2+\mathrm{dx} ; \mathrm{y}=\mathrm{y} 2+\mathrm{dy} ; \mathrm{z}=\mathrm{z} 2+\mathrm{dz}$;

$n d x=\operatorname{Box} 2(x, y, z, d x, d y, d z, n d x$, typ $, Q, f p 1)$;

$\mathrm{x}=\mathrm{x} 2+\mathrm{dx} ; \mathrm{y}=\mathrm{y} 2+\mathrm{dy} ; \mathrm{z}=\mathrm{z} 2-\mathrm{dz}$;

$n d x=\operatorname{Box} 2(x, y, z, d x, d y, d z, n d x$, typ $, Q, f p 1)$;

$\mathrm{x}=\mathrm{x} 2+\mathrm{dx} ; \mathrm{y}=\mathrm{y} 2-\mathrm{dy} ; \mathrm{z}=\mathrm{z} 2+\mathrm{dz}$;

$n d x=\operatorname{Box} 2(x, y, z, d x, d y, d z, n d x$, typ $, Q, f p 1)$;

$\mathrm{x}=\mathrm{x} 2+\mathrm{dx} ; \mathrm{y}=\mathrm{y} 2-\mathrm{dy} ; \mathrm{z}=\mathrm{z} 2-\mathrm{dz}$;

$n d x=\operatorname{Box} 2(x, y, z, d x, d y, d z, n d x$, typ $, Q, f p 1)$; 


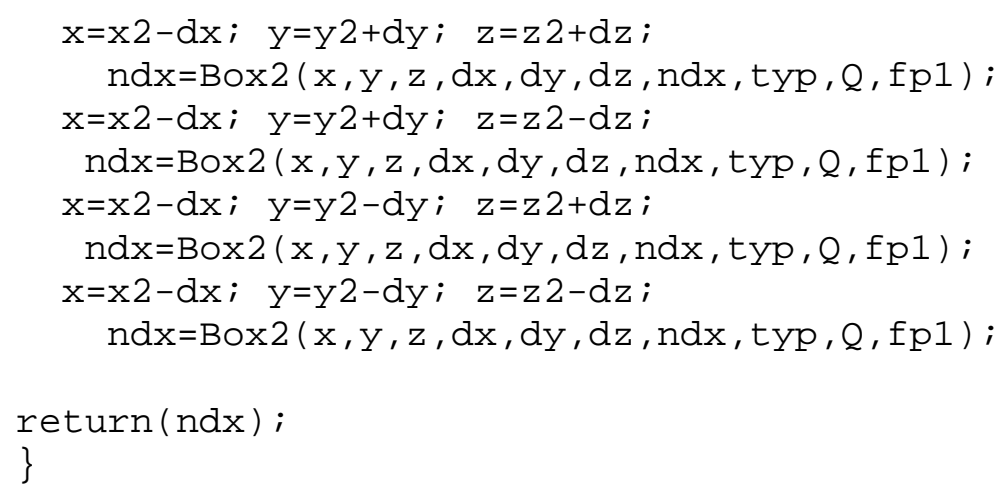




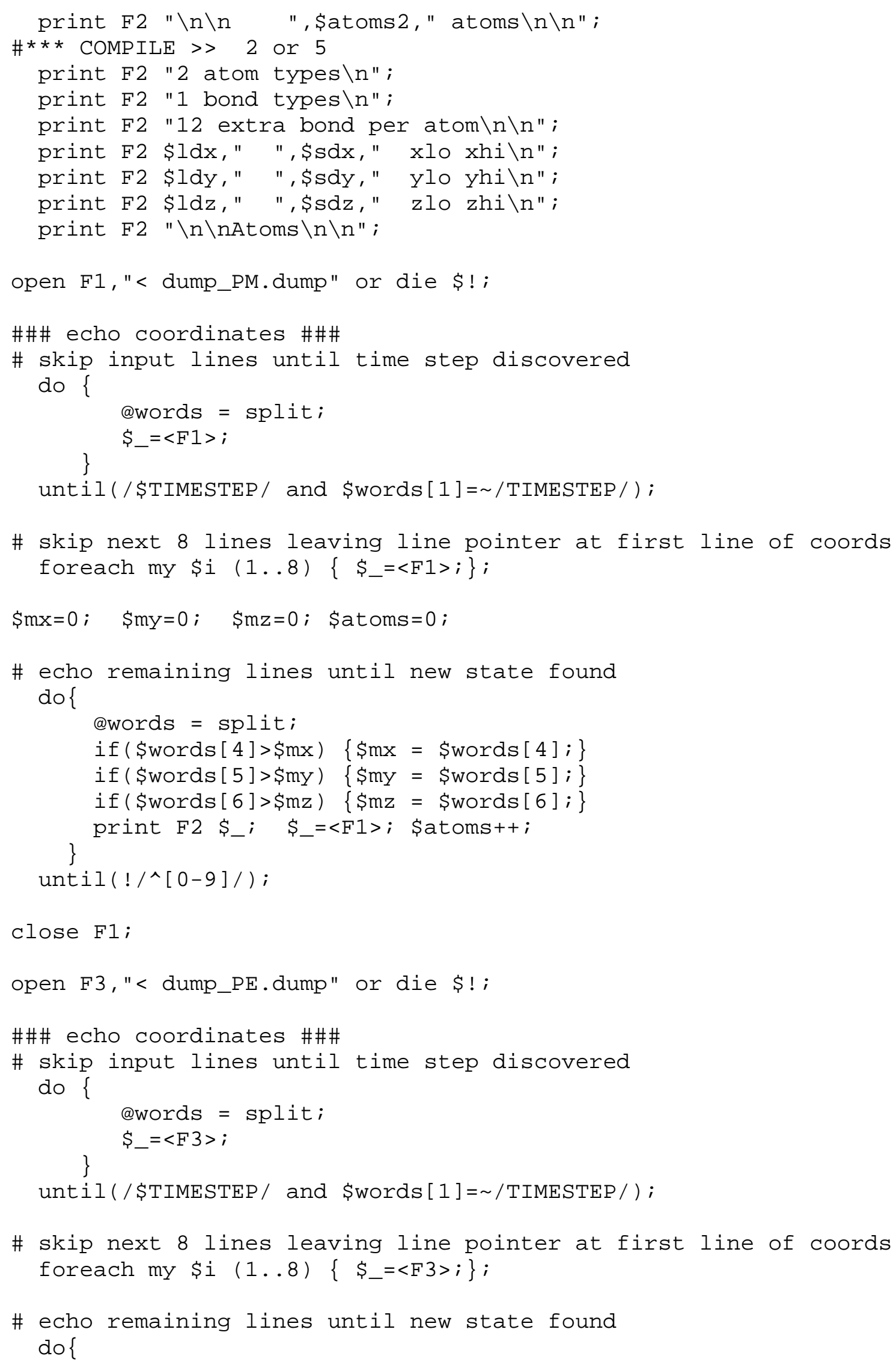




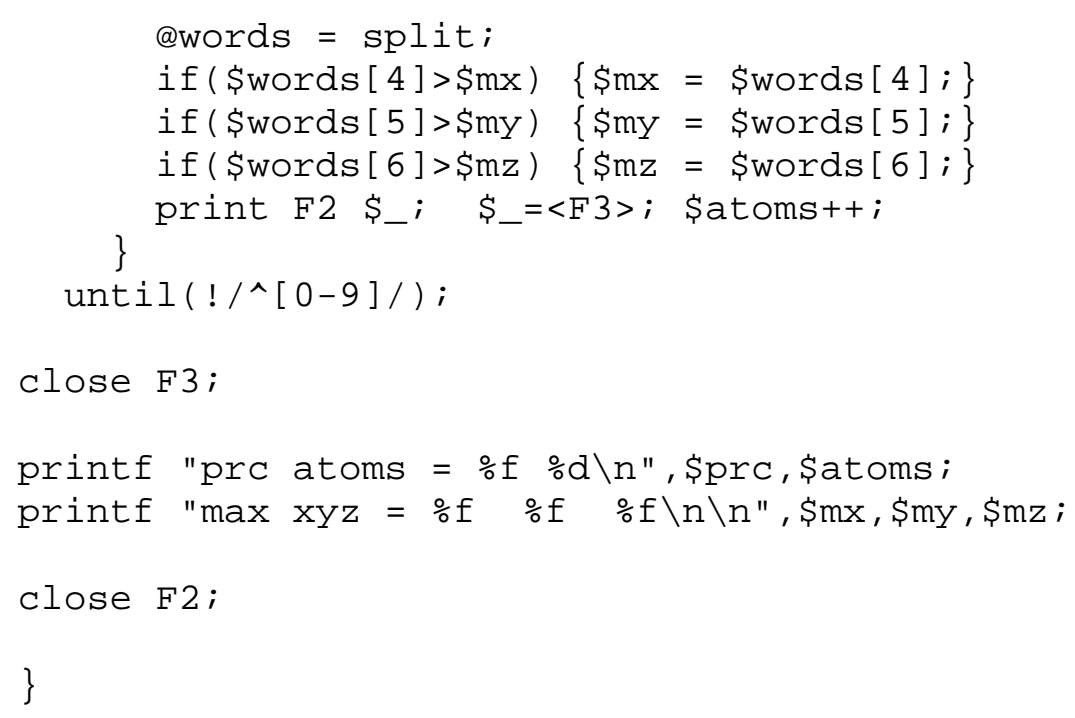




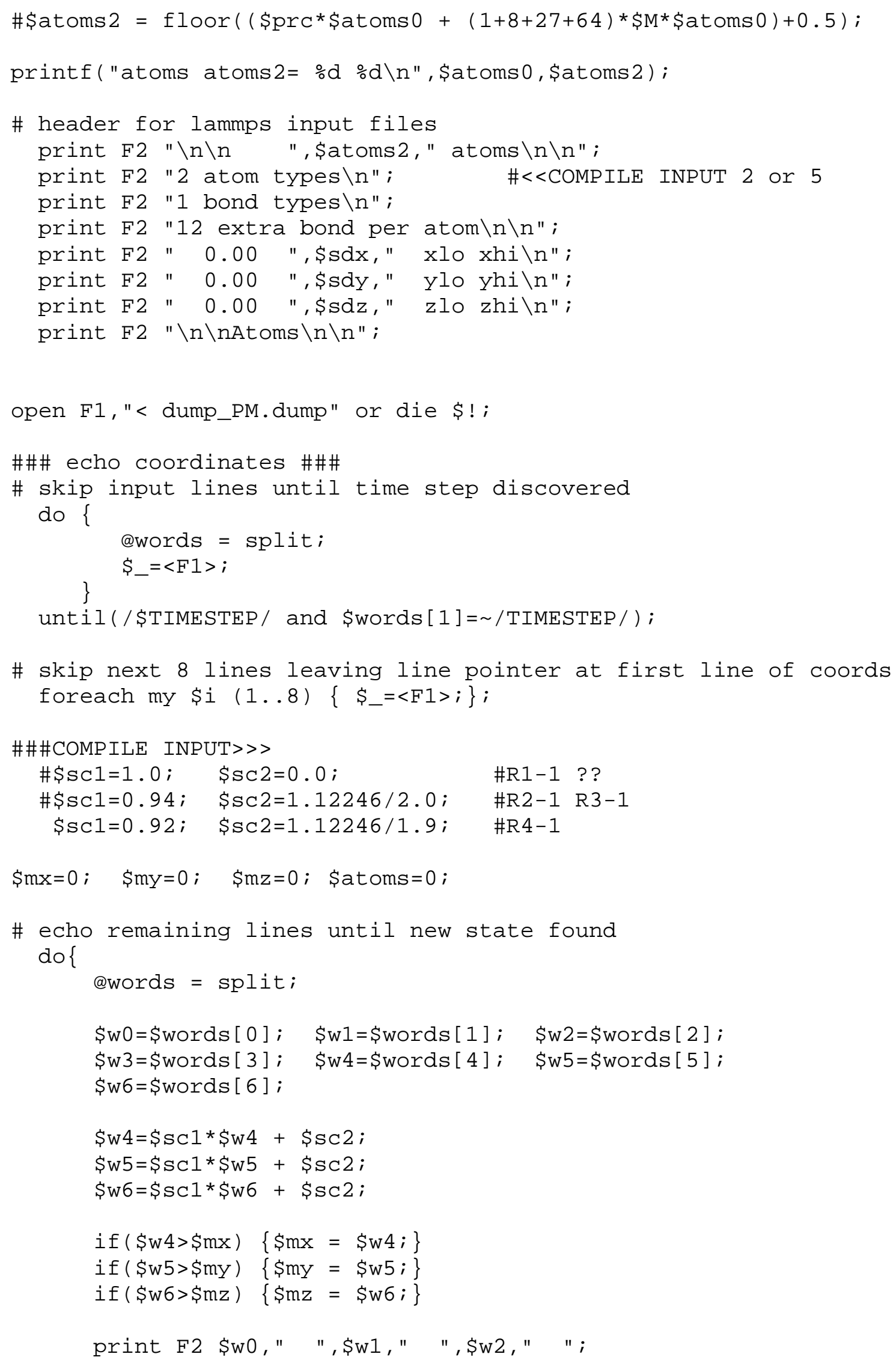




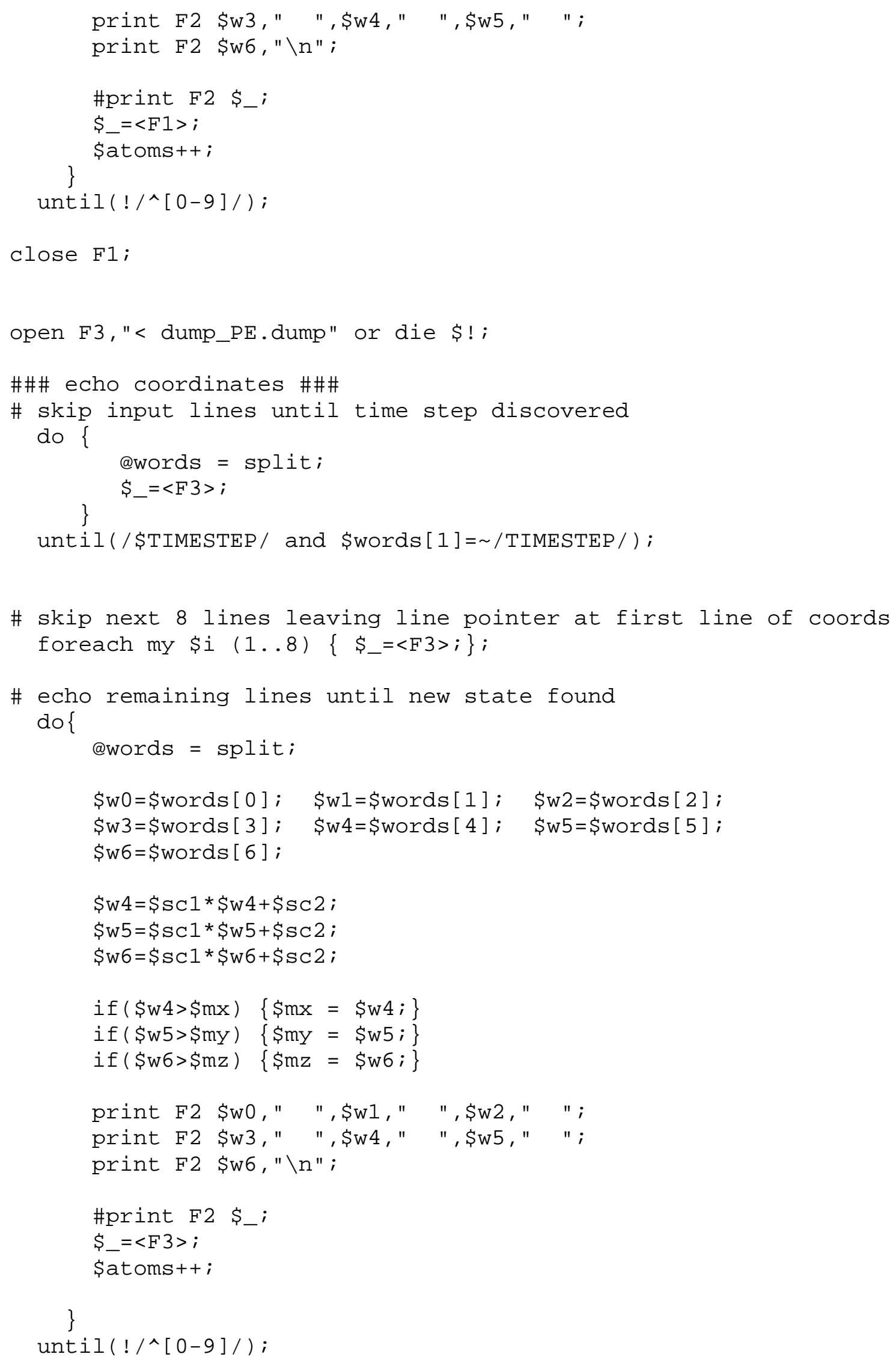




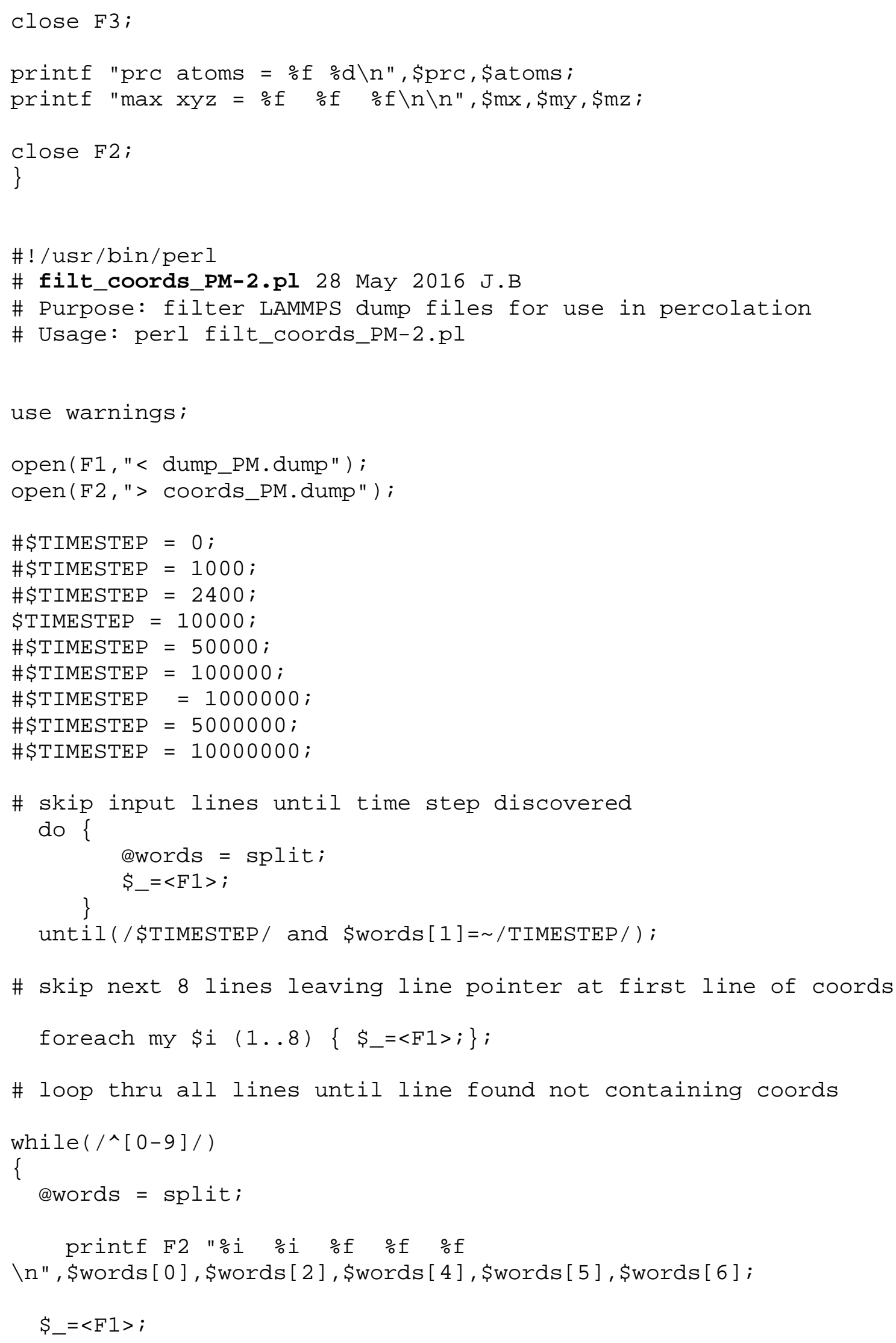


close F1; close F2;

\#!/usr/bin/perl

\# filt_coords_PE-2.pl 28 May 2016 J.B

\# purpose: filter LAMMPS dump files for use in percolation programs

\# usage: \# perl filt_coords_PE-2.pl

\# UNIX command to sort sort $-n<$ outfile $>$ sorted file

\# input: 1. compiletime time-step

\# 2. lammps dumpfile from NVT or NPT

\# output: ascii file with 5 columns: id type $x, y, z$

use warnings;

open (F1, "< dump_PE.dump");

open(F2,"> coords_PE.dump");

\#\$TIMESTEP $=0$;

\#\$TIMESTEP $=1150 ;$

$\#$ \$TIMESTEP $=2400 ;$

$\$$ TIMESTEP $=10000 ;$

\#\$TIMESTEP $=50000 ;$

\#\$TIMESTEP $=100000 ;$

$\#$ \#TIMESTEP $=500000 \%$

$\#$ \$TIMESTEP = $10000000 ;$

$\#$ \$TIMESTEP $=5000000 ;$

$\#$ \$TIMESTEP $=10000000 ;$

\# skip input lines until time step discovered do \{

@words = split;

\}

$\$=<F 1>$;

until(/\$TIMESTEP/ and \$words[1]= /TIMESTEP/);

\# skip next 8 lines leaving line pointer at first line of coords

foreach my $\$ \mathrm{i}(1 . .8)\left\{\$_{-}=<\mathrm{F} 1>;\right\}$;

\# loop thru all lines until line found not containing coords

while $(/ \wedge[\odot-9] /)$

\{

@words = split

printf $F 2$ "\%i $\% i \quad \% f \quad \% f \quad \% f$

$\backslash \mathrm{n} "$, \$words [0], \$words [2], \$words [4], \$words [5], \$words [6];

$\$+=\mathrm{F} 1>$; 


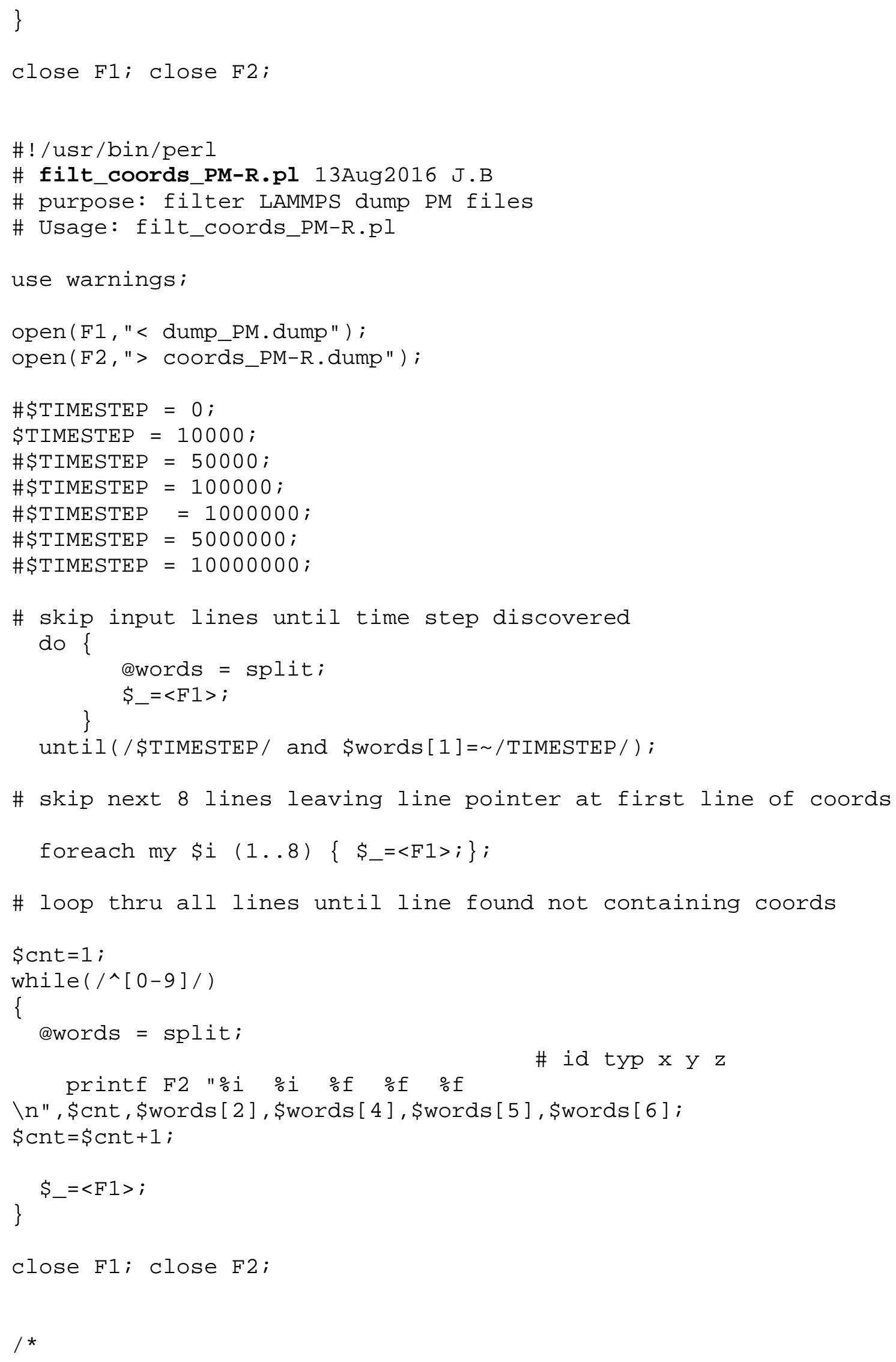


dist3.c 13Aug2016 J.Bedard

Purpose: compute distance between all atoms and output bond pairs list Usage: dist3. c < Rm> */

\# include <stdio.h>
\# include <stdlib.h>
\# include <math.h>
\# include <string.h>

int main(int argc, char *argv[])\{

FILE *fp1; fp1=fopen("coords_PM-R.dump", "r");

FILE *fp2; fp2=fopen("bonds_PM.dump", "w");

double $c[50000][4] ; / * 0.14999$ \#atoms, [id] [type, $x, y, z]$

int cnt, atoms, id;

int $i, j$;

float $\quad x, y, z$, typ; $\quad /$ scanf reads float only */

double $x 2, y 2, z 2$;

double D, D2, Rmin, Rmax;

char str[20], cv;

Rmin=1 . 0 ;

strcpy(str, argv[1]); $\operatorname{Rmax}=($ double) atof(str);

/*** get coords LOAD Crd[id][typ x y z $]$ ***/

$/$ * first index is atom id */

atoms $=1 ; \mathrm{cV}=$ 'A';

while ( cV ! =EOF)

\{

$c v=f s c a n f(f p 1, " \% d \% f \% f \% f \% f \backslash n ", \& i d, \& t y p, \& x, \& y, \& z)$;

$C[$ atoms $][\Theta]=($ double $)$ typ;

$C[$ atoms $][1]=($ double $) x$;

$C[$ atoms $][2]=($ double $) y$;

$c[$ atoms $][3]=($ double $) z$;

\}

atoms++;

atoms=atoms -2 ;

printf( "\%d\n", atoms );

$l^{*}$ for $(i=1 ; i<=$ atoms $; i++)$

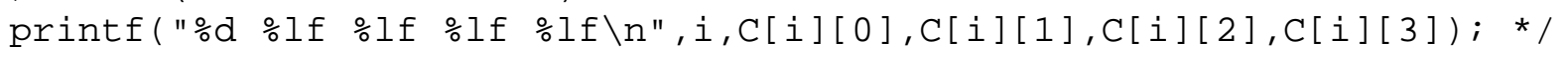

cnt $=1$;

for $(i=1 ; i<=$ atoms $-1 ; i++)$

\{

for $(j=i+1 ; j<=$ atoms $; j++)$

\{

$x 2=\operatorname{pow}(C[i][1]-C[j][1], 2) ;$ 


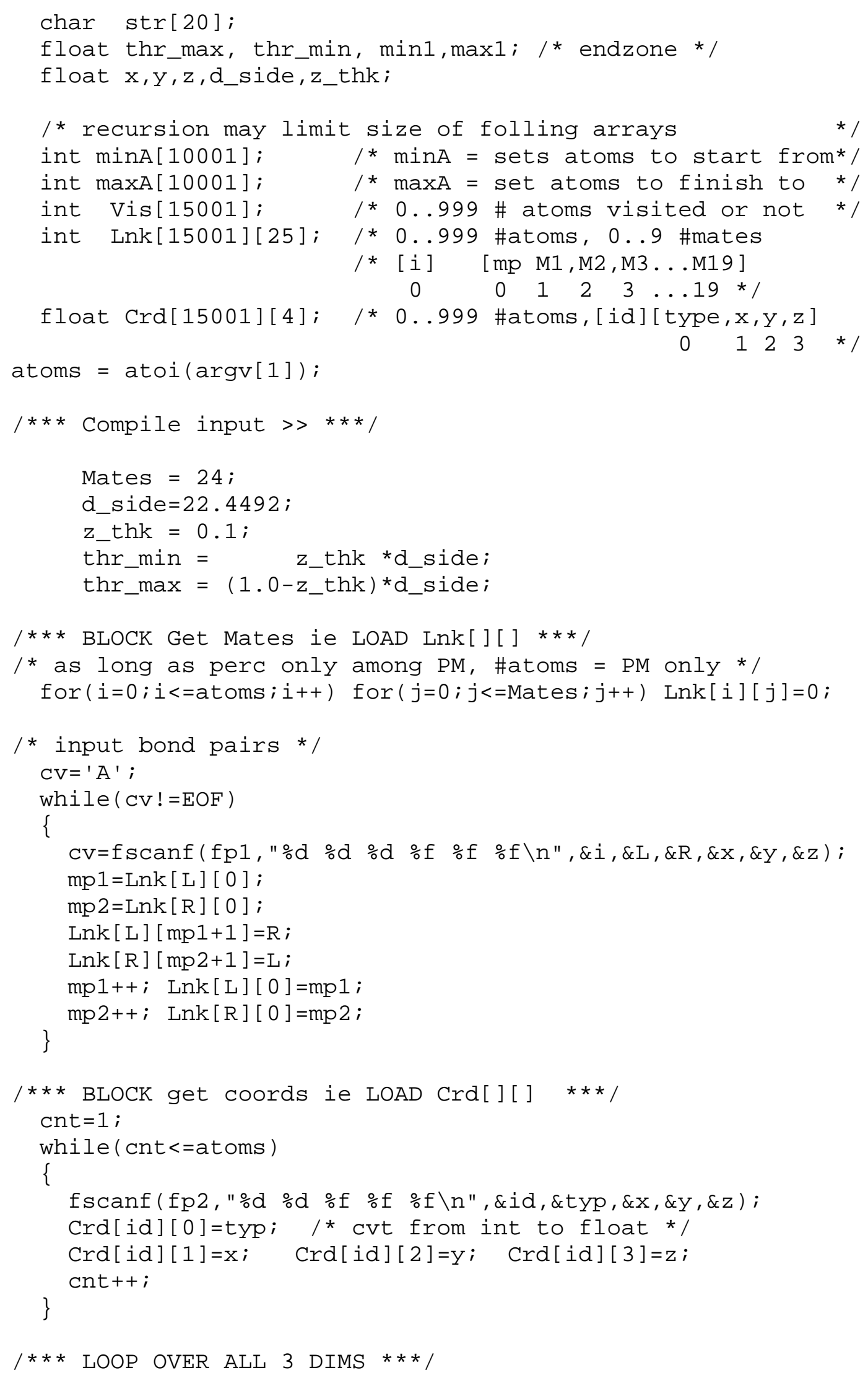




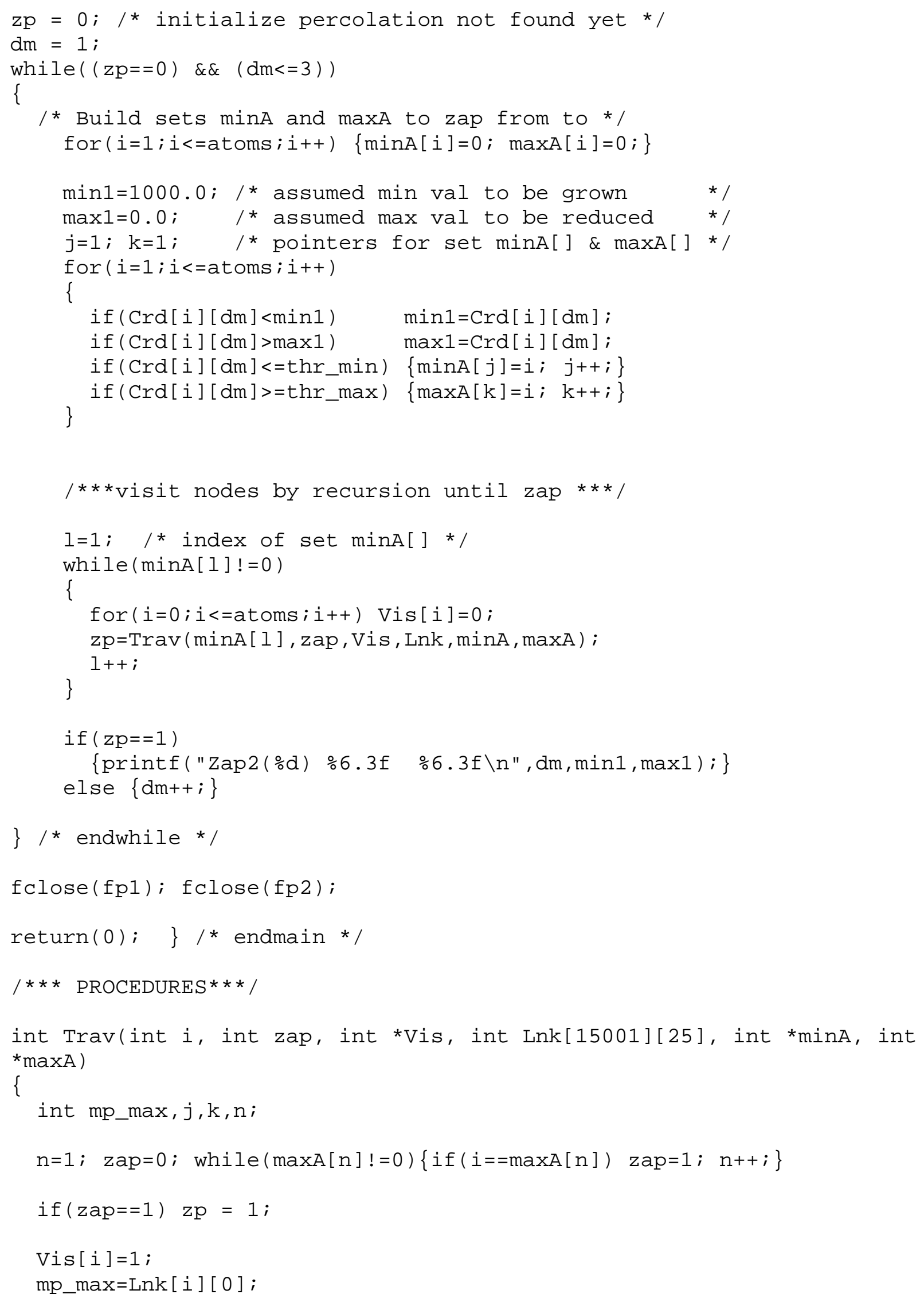




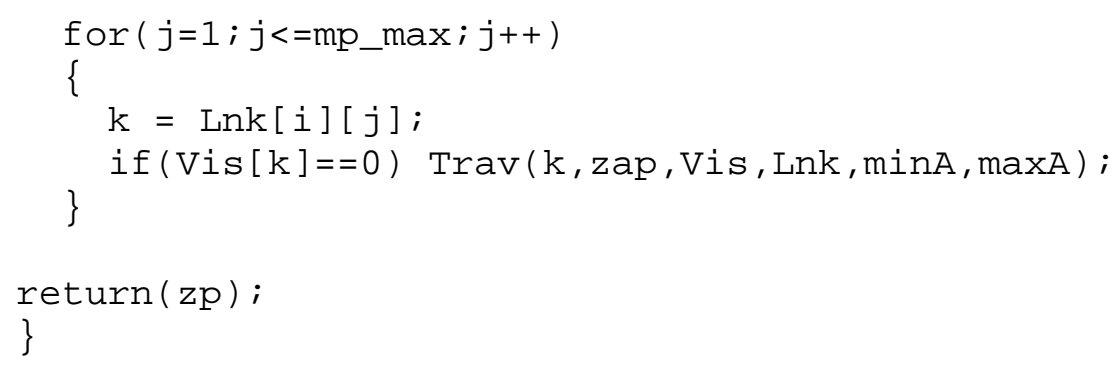




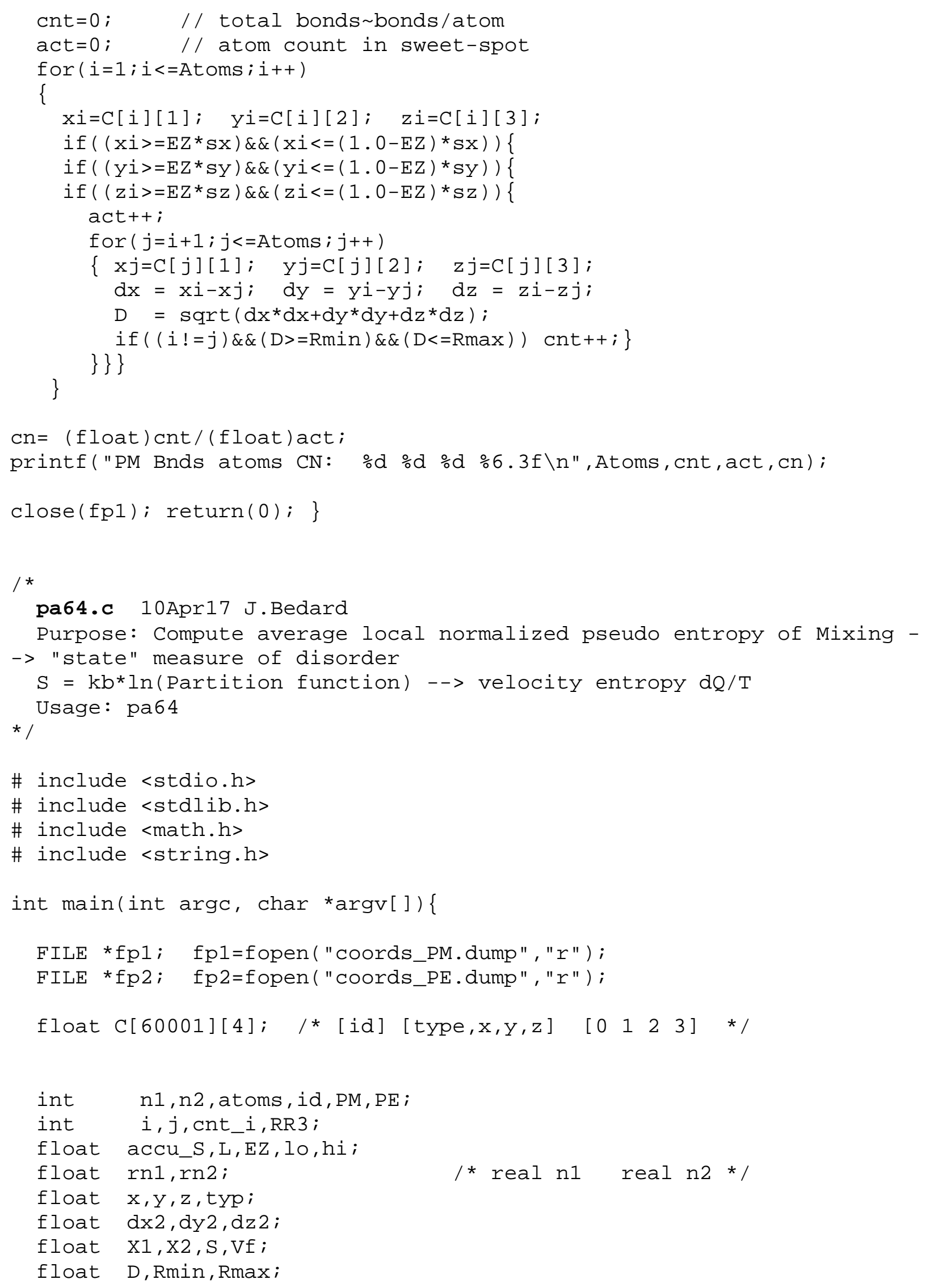




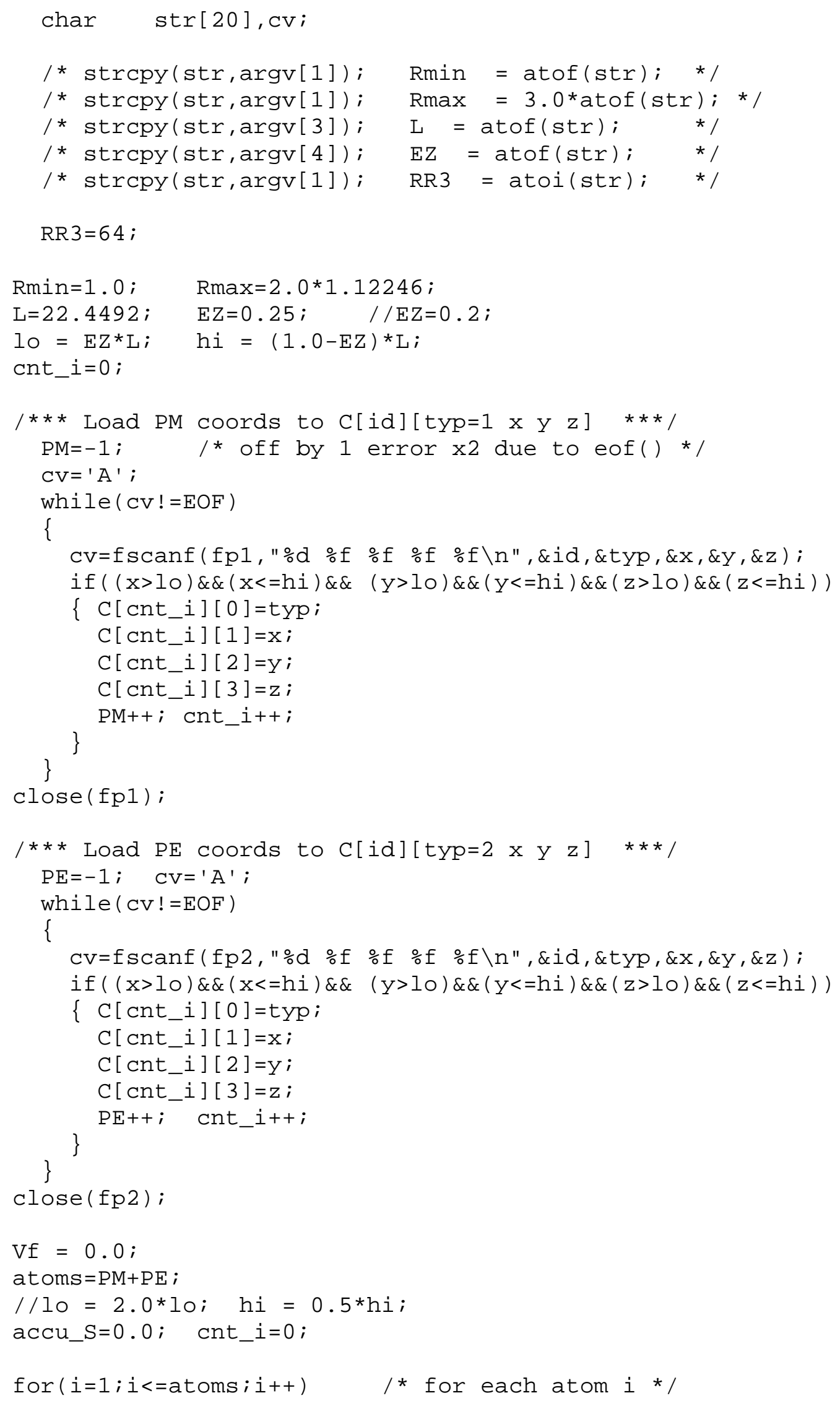


I* count all atoms within Rmax of atom $i$ *

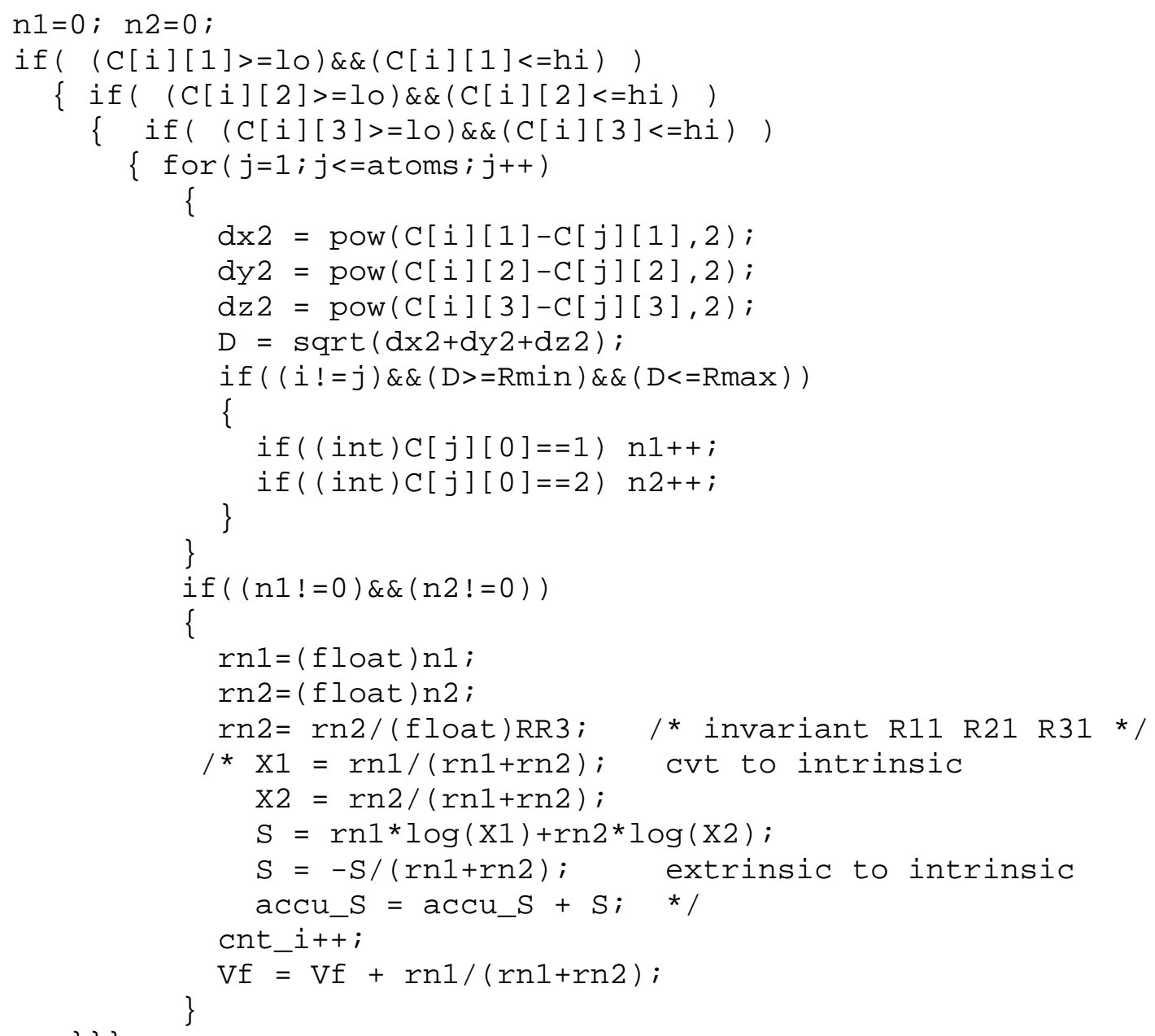


Appendix B. Programs used in hard-shell modeling for particle deformation (Chapter 3)

The following list is one example of the order in which the programs in this appendix were run to produce the output for deformable $\mathbf{H}$ and $\mathbf{E}$ phase particles simulations per this paper. All programs in this appendix were run on a model ASUS 2.53 GHZ laptop running 64 bit Windows 7 Home Premium 2009. LAMMPS @ was run on the West Virginia University High Performance Cluster running a UNIX operating system.

Order of execution programs used in study of particle deformation per pseudo-sintering

Programs filt_coord_PM-2.pl and filt_coord_PE-2.pl are the same as those listed in appendix A.

$<\mathrm{p}>$ is an inline parameter standing for volume fraction $=$ number fraction $=$ number fraction $=\mathrm{N}_{\mathrm{H}} /$ $\left(\mathrm{N}_{\mathrm{H}}+\mathrm{N}_{\mathrm{E}}\right)$.

$<$ ts $>$ stands for time step in Leonard-Jones units. $t s=3800$ timesteps from most results in this study.

$\underline{\text { Run program }}$

1. filt_coords_PM-2.pl

2. filt_coords_PE-2.pl

3. glob3.c $<\mathrm{p}>$

4. lammps < input_MIX7.txt

5. lammps < input_redump.txt

6. filt_coords_PM-mol-R.pl

7. filt_coords_PE-mol.pl

8. $\quad$ Rg3.pl $<\mathrm{ts}><\mathrm{p}>$

9. $\operatorname{dist9M.c~}<\mathrm{p}>$ $\underline{\text { Explanation }}$

Strip unnecessary information from data file.

Strip unnecessary information from data file.

Replace all H coordinates with 125 point cubes

Run Lammps. See appendix C for MIX7 script.

Remove all but coordinates from single timestep.

Strip away unnecessary information from data file.

Strip away unnecessary information from data file.

Compute table of $\mathbf{R}_{\mathbf{g}}$ values for each cluster.

Create file of bond pairs between molecules
Page

App. A

App. A

108 
10. ZapM.c $<\mathrm{p}>$

11. CNHH-M.c

12. CNHE-M.c
Recurse through bond pairs and test for percolation

Compute c.n. between $\mathrm{H}$ phase clusters

Compute c.n. between $\mathrm{H}$ and $\mathrm{E}$ hard-shell spheres
121

124

126

\section{References}

1. E.J. Barbero, A.J. Bedard (2017) Electrical percolation threshold of magnetostrictive inclusions in a piezoelectric matrix composite as a function of relative particle size. Computational Particle Mechanics (printed online 25 July 2017 ISSN 2196-4378)

2. D. Stauffer, A. Aharmoney (1994) Introduction to Percolation Theory. $2^{\text {nd }}$ Ed. Taylor and Francis, London UK.

3. R.L. Kruse (1989) Programming with data structures, Prentice Hall, Chap. 17

4. S.J.Plimpton (1995) LAMMPS @ Fast parallel algorithms for short-range molecular dynamics. J. Comp. Phys. 117:1-19 
$\underline{\text { Programs used in Chapter } 3}$

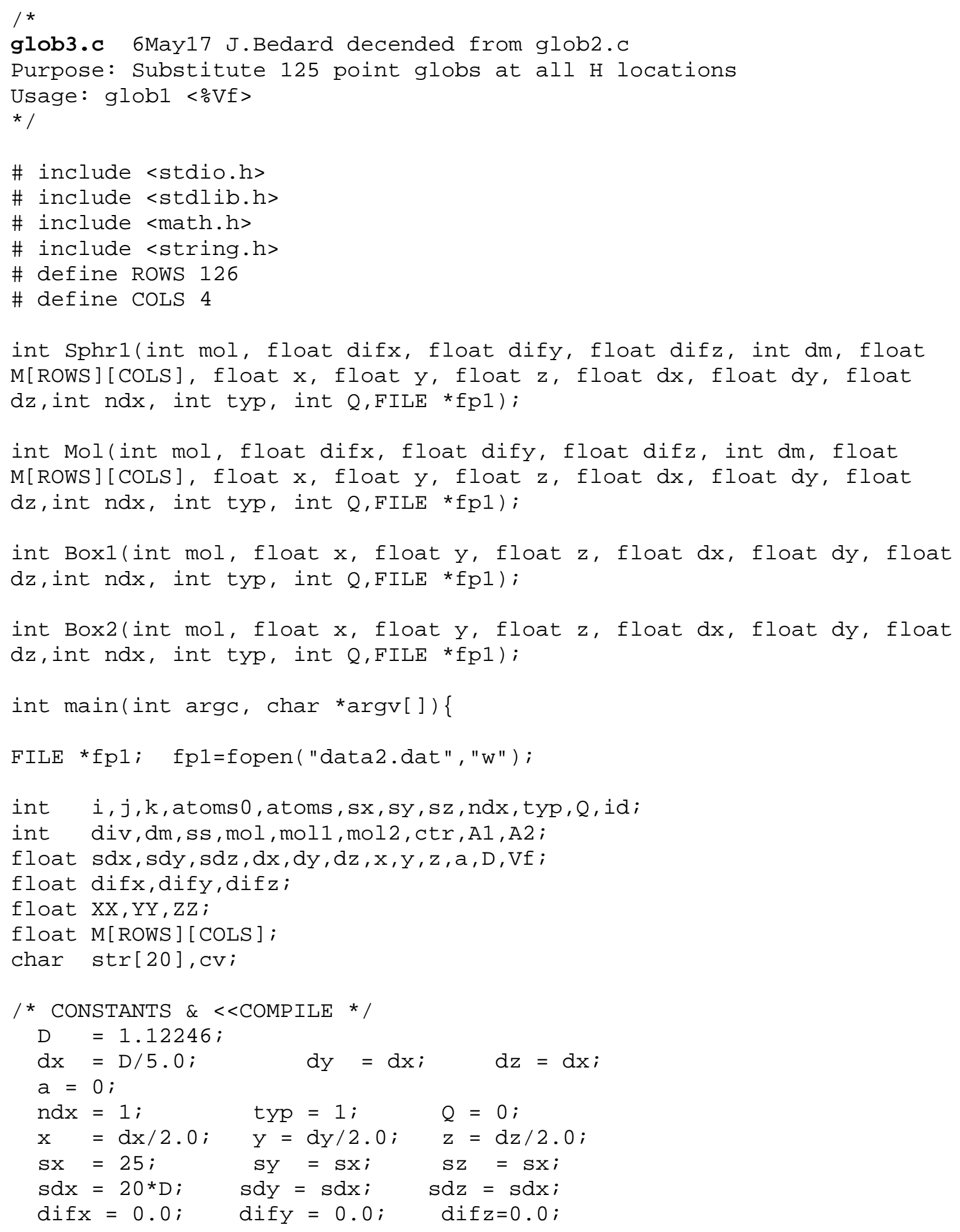




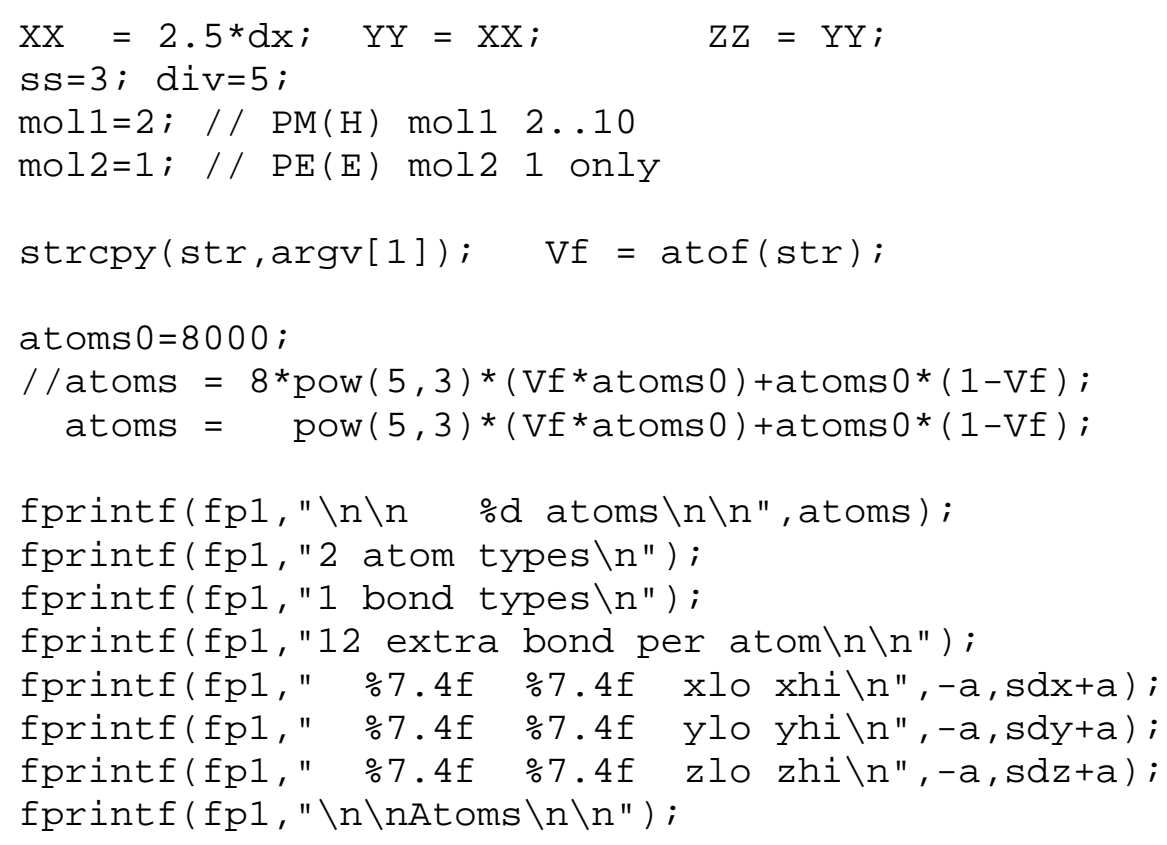




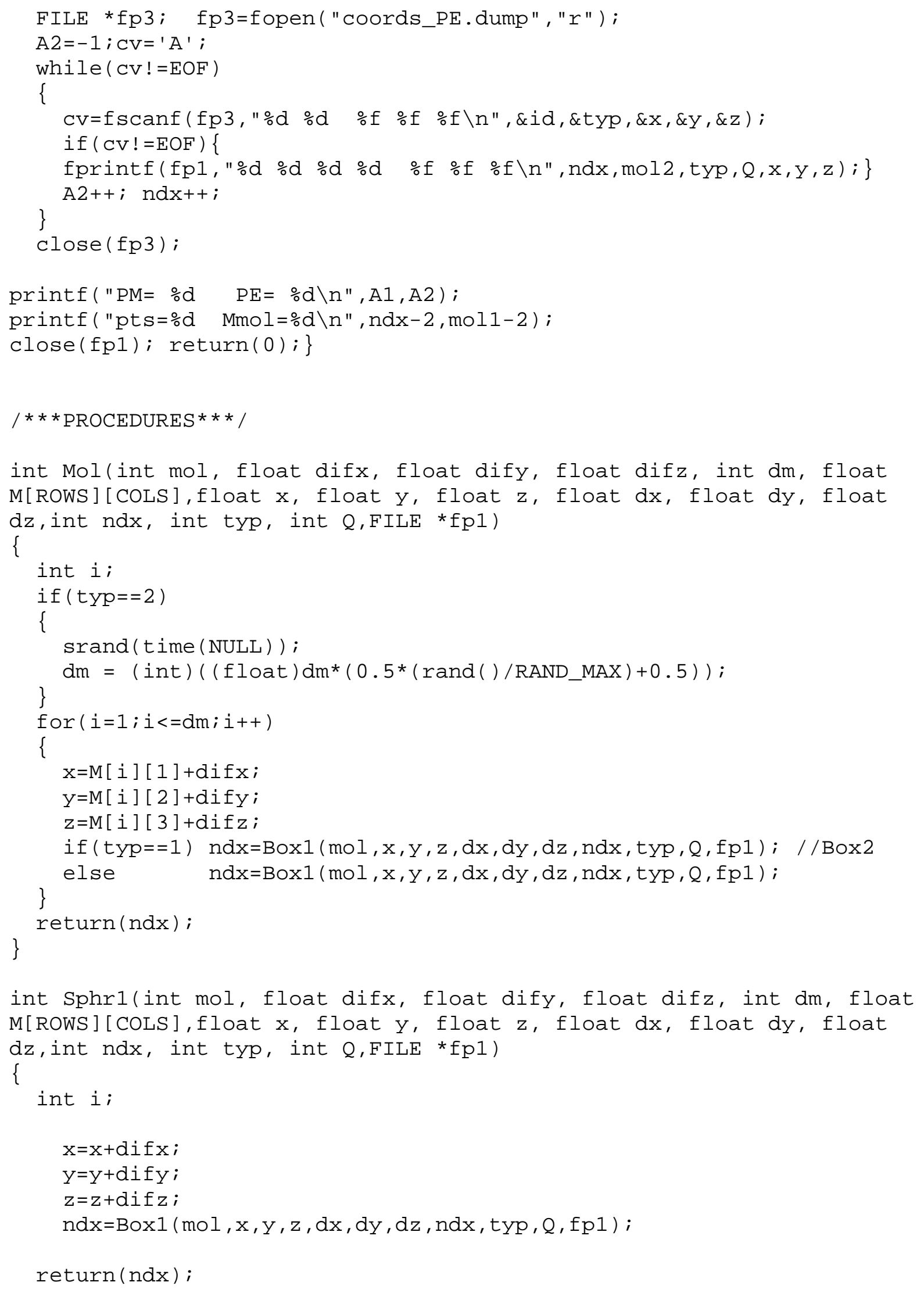


int Box1(int mol, float $x$, float $y$, float $z$, float $d x$, float dy, float $\mathrm{dz}$, int ndx, int typ, int $Q$, FILE ${ }^{*} \mathrm{fp} 1$ )

\{

fprintf(fp1, "\%d \%d \%d \%d \%f \%f \%f\n", ndx, mol, typ, $Q, x, y, z)$; $n d x++$; \} $\operatorname{return}(n d x)$;

int Box2(int mol, float $x$, float $y$, float $z$, float $d x$, float dy, float $\mathrm{dz}$, int $\mathrm{ndx}$, int typ, int $\mathrm{Q}$, FILE $\left.{ }^{*} \mathrm{fp} 1\right)$ \{

int $i, j, k$;

float $x 2, y 2, z 2$;

$\mathrm{x} 2=\mathrm{x} ; \mathrm{y} 2=\mathrm{y} ; \mathrm{z} 2=\mathrm{z} ; \quad /$ * always adjust from initial $\mathrm{xyz}$ */ $\mathrm{dx}=\mathrm{dx} / 4.0 ; \quad \mathrm{dy}=\mathrm{dy} / 4.0 ; \mathrm{dz}=\mathrm{dz} / 4.0$;

$x=x 2+d x ; y=y 2+d y ; z=z 2+d z ;$

fprintf(fp1, "\%d \%d \%d \%d \%f \%f \%f\n", ndx, mol, typ, $Q, x, y, z)$; $n d x++; \quad x=x 2+d x ; y=y 2+d y ; z=z 2-d z$;

fprintf(fp1, "\%d \%d \%d \%d \%f \%f \%f\n", ndx, mol, typ, $Q, x, y, z)$; $n d x++; \quad x=x 2+d x ; y=y 2-d y ; z=z 2+d z ;$

fprintf(fp1, "\%d \%d \%d \%d \%f \%f \%f\n", ndx, mol, typ, $Q, x, y, z)$; $n d x++; x=x 2+d x ; y=y 2-d y ; z=z 2-d z$;

fprintf(fp1, "\%d \%d \%d \%d \%f \%f \%f\n", ndx, mol, typ, $Q, x, y, z)$;

$n d x++; \quad x=x 2-d x ; y=y 2+d y ; z=z 2+d z ;$

fprintf(fp1, "\%d \%d \%d \%d \%f \%f \%f\n", ndx, mol, typ, $Q, x, y, z)$; $n d x++; \quad x=x 2-d x ; y=y 2+d y ; z=z 2-d z$;

fprintf(fp1, "\%d \%d \%d \%d \%f \%f \%f\n", ndx, mol, typ, $Q, x, y, z)$; $n d x++; \quad x=x 2-d x ; y=y 2-d y ; z=z 2+d z$;

fprintf(fp1, "\%d \%d \%d \%d \%f \%f \%f\n", ndx, mol, typ, $Q, x, y, z)$; $n d x++; x=x 2-d x ; y=y 2-d y ; z=z 2-d z$;

fprintf(fp1, "\%d \%d \%d \%d \%f \%f \%f\n", ndx, mol, typ, $Q, x, y, z)$; $n d x++$;

$\operatorname{return}(n d x)$;

\}

\#! /usr/bin/perl

\# filt_coords_PM-mol-R.pl (renumbered) 22Feb17 J.B

\# purpose: filter dump attimestep for dist5M.c mol bonds list \# $\quad$ OUTPUT HAS MOLECUL FIELD--BUT PAPR1 FILT PROGS DO NOT

\# usage: filt_coords_PM-mol-R.pl <timestep>

use warnings;

\$TIMESTEP $=$ \$ARGV $[0] ;$ 


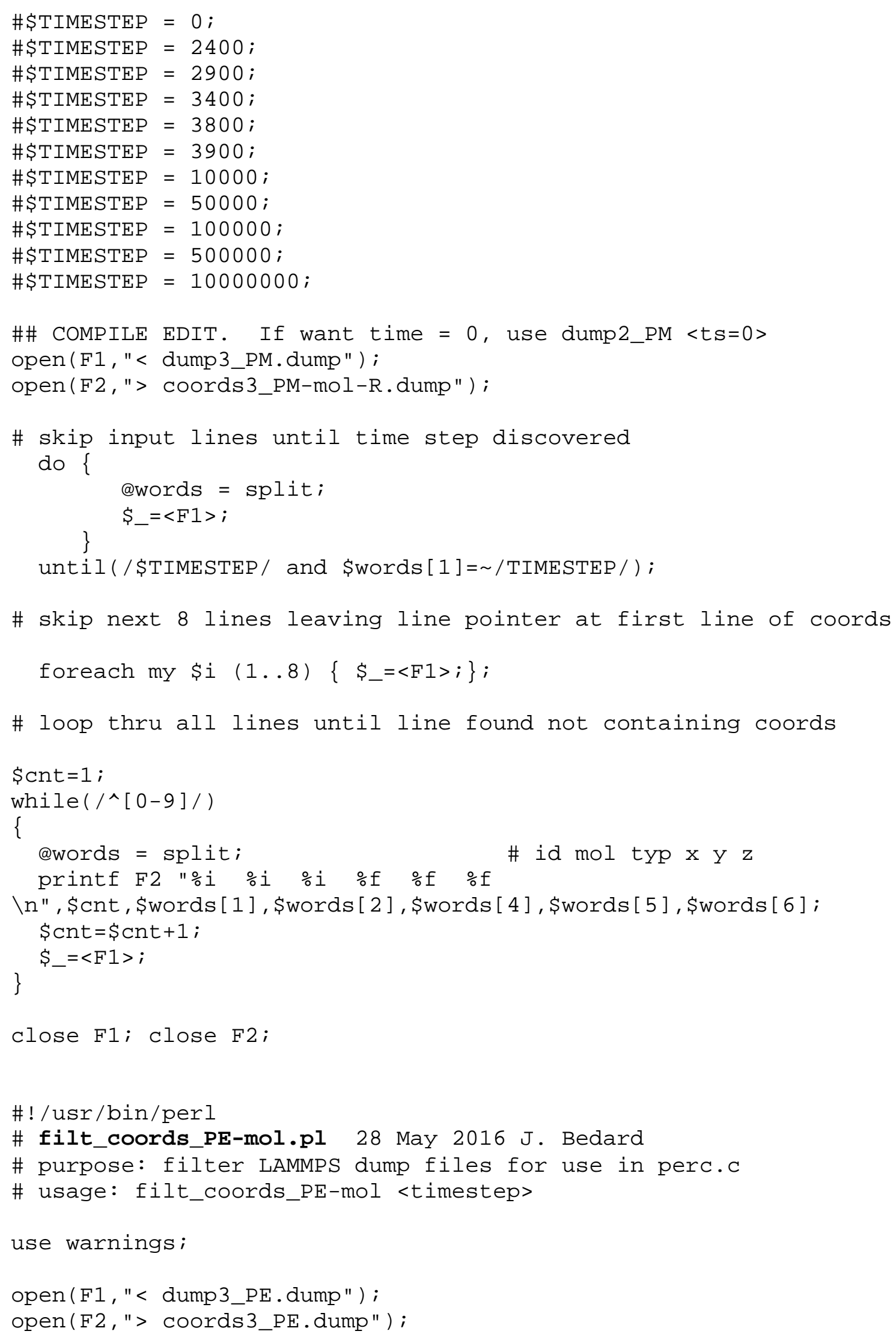




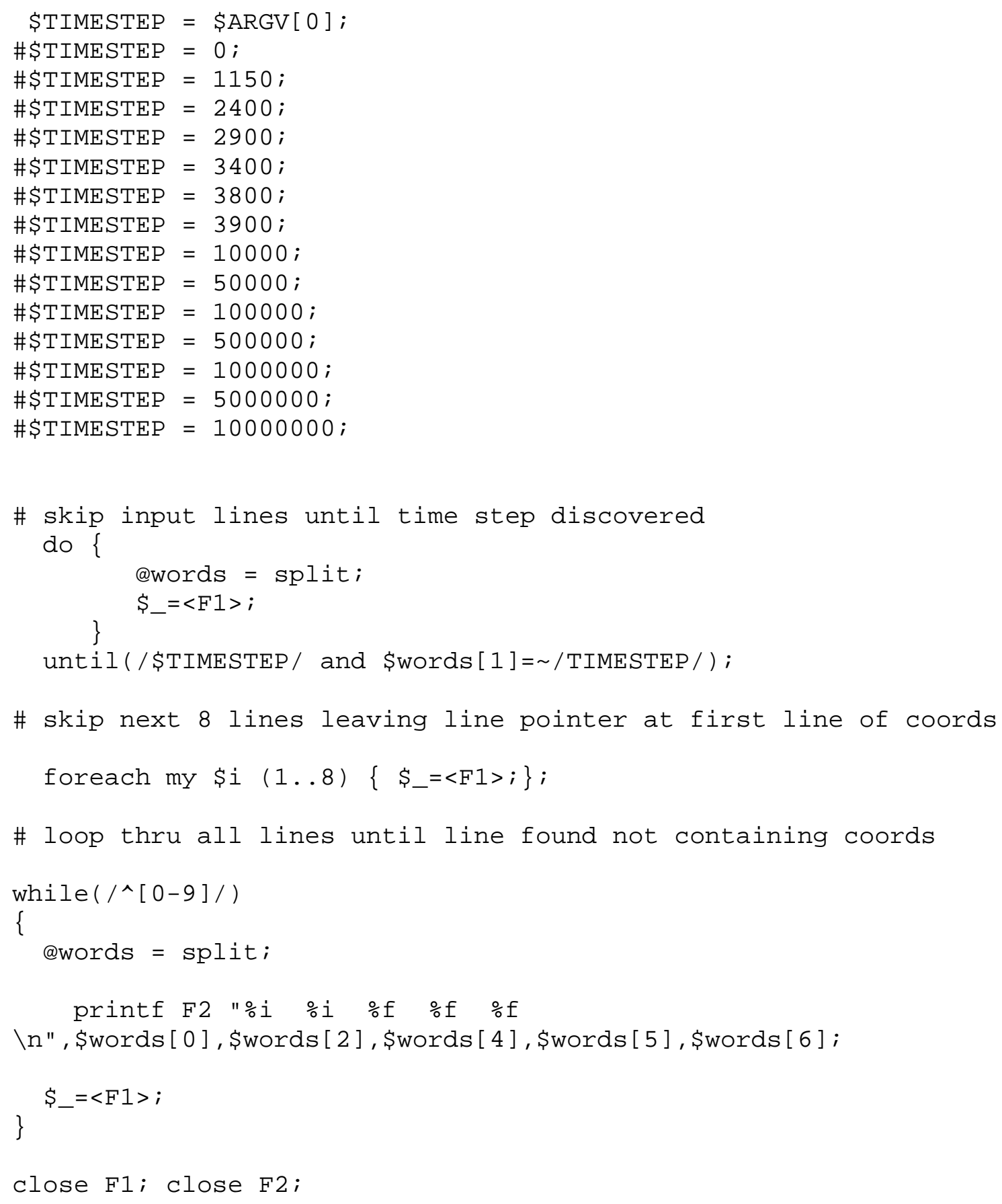




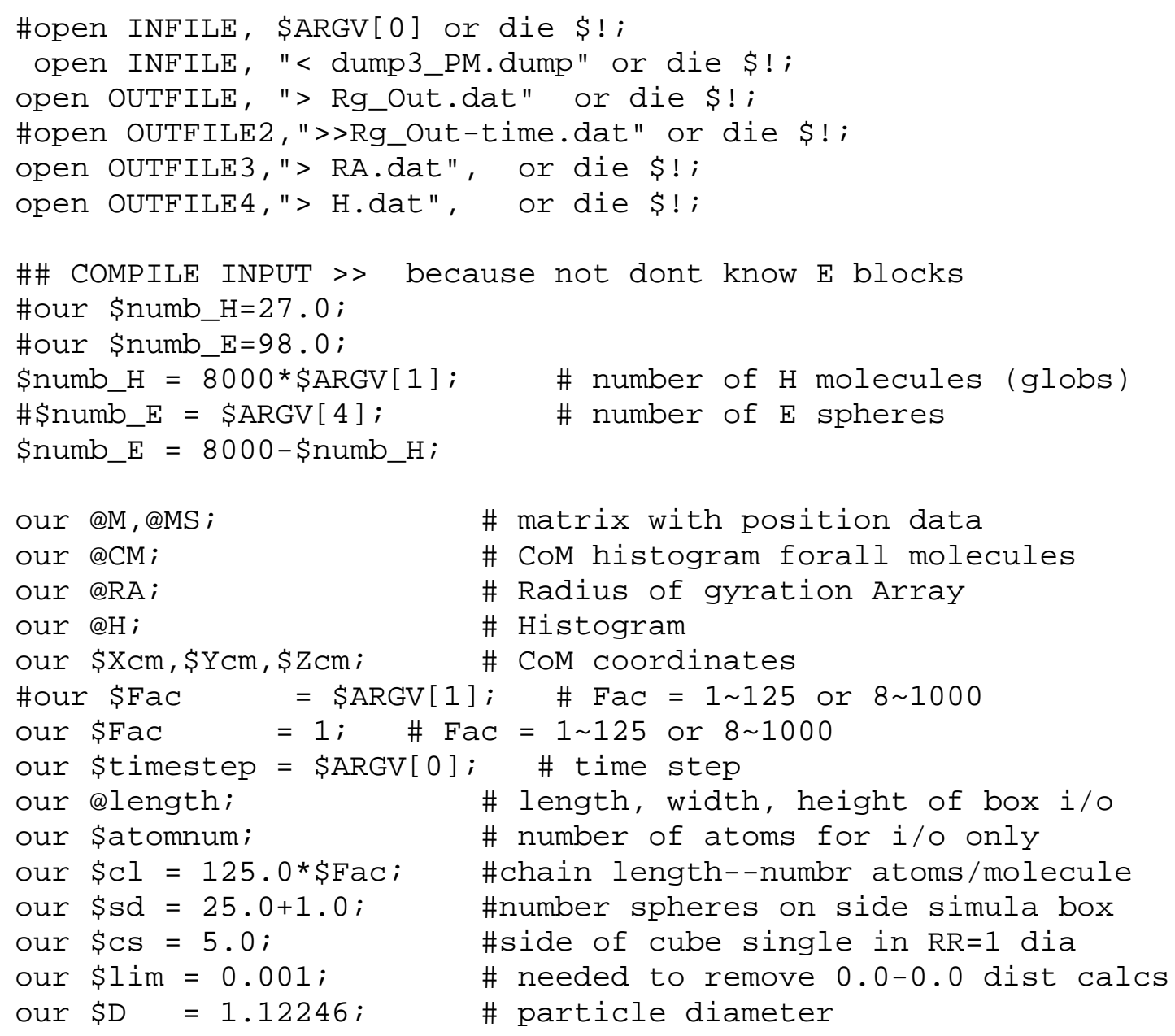

\#\#\# BLOCK LOAD ARRAY M WITH ATOM DATA \#\#\#

\# skip lines until time step is found while $(!(\$+=\sim /$ timestep/ $)) \quad\{\$+=<$ INFILE $>;\}$

\# get dims of box and skip lines to get to position data $\$_{-}=\langle$INFILE $>$; $\#$ skip item2

$\$ \_=\langle\text {INFILE }>\text {; }$

my @numline = split;

\$atomnum = \$numline $[0]$;

$\$_{-}=<$INFILE $>$; skip item3

$\$_{-}=<$INFILE>;

my @boxline = split;

\$length $[0]=$ \$boxline[1] - \$boxline [0];

$\$_{-}=\langle$INFILE $>$;

my @boxline = split;

\$length[1] = \$boxline[1] - \$boxline[0];

$\$_{-}=<$INFILE $>$; 


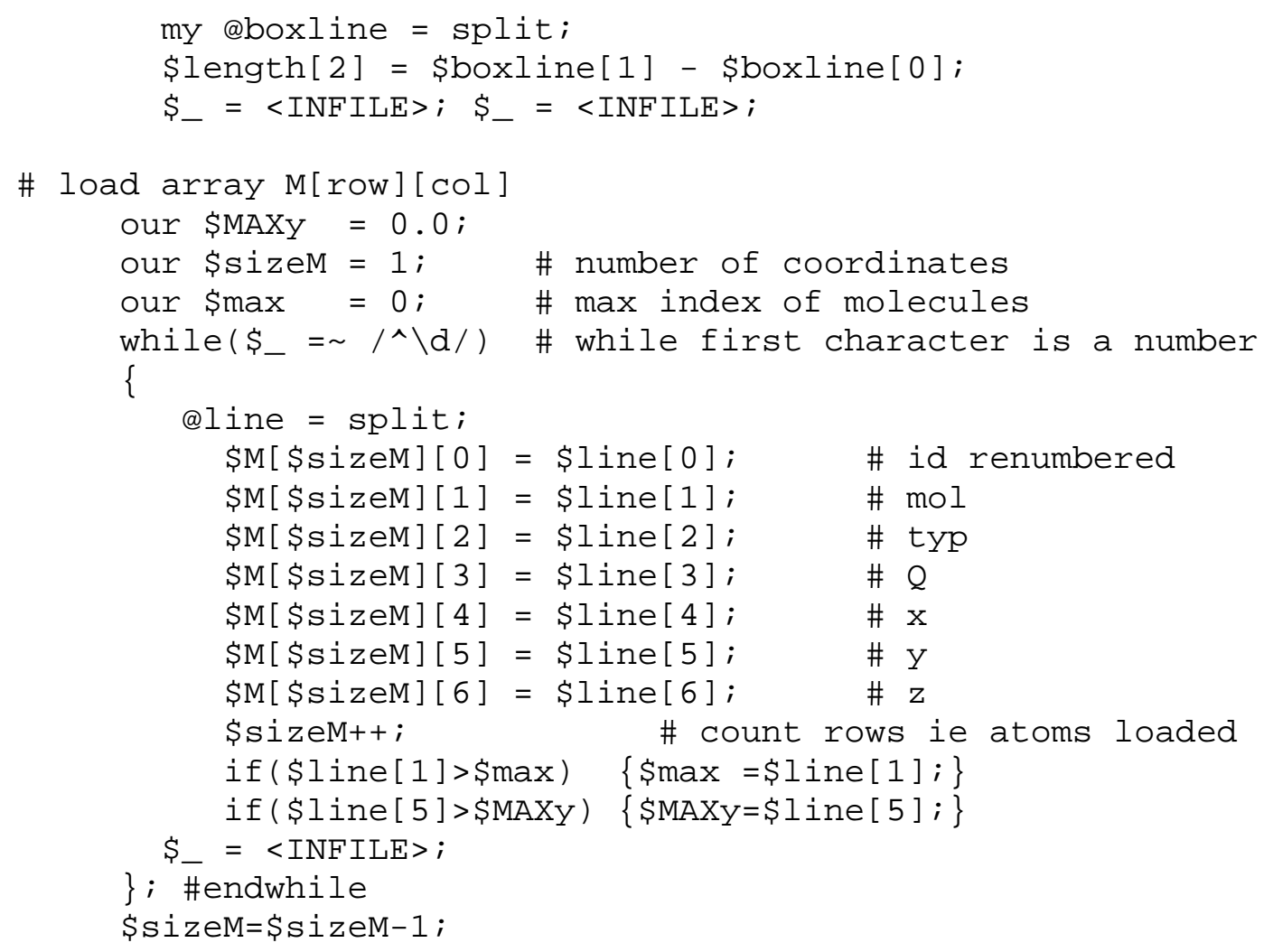




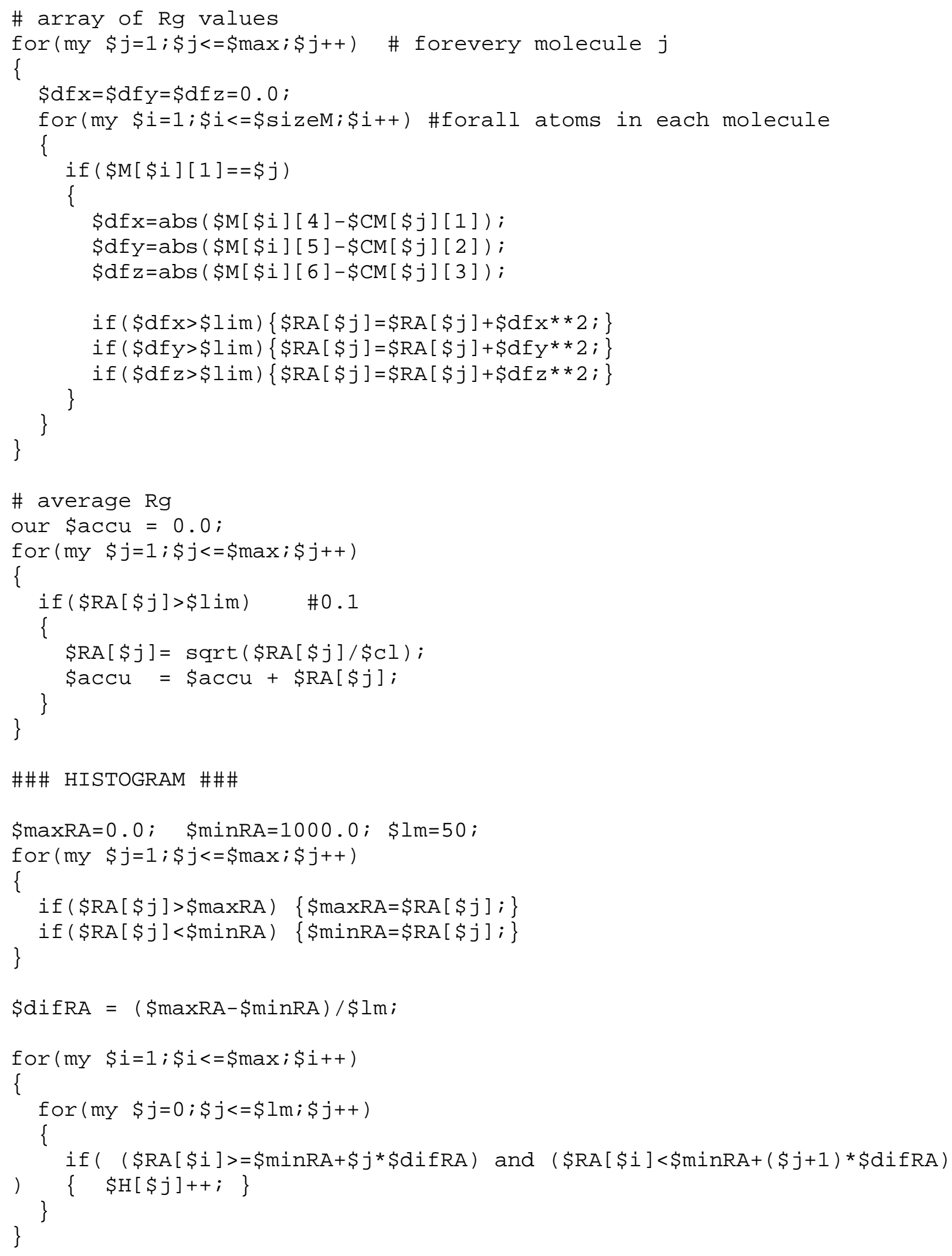


for (my $\$ j=0.55 * \$ 1 m ; \$ j<=\$ 1 m+1 ; \$ j++)$ \{printf OUTFILE4 "\%6.3f

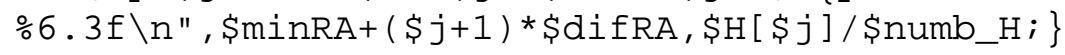

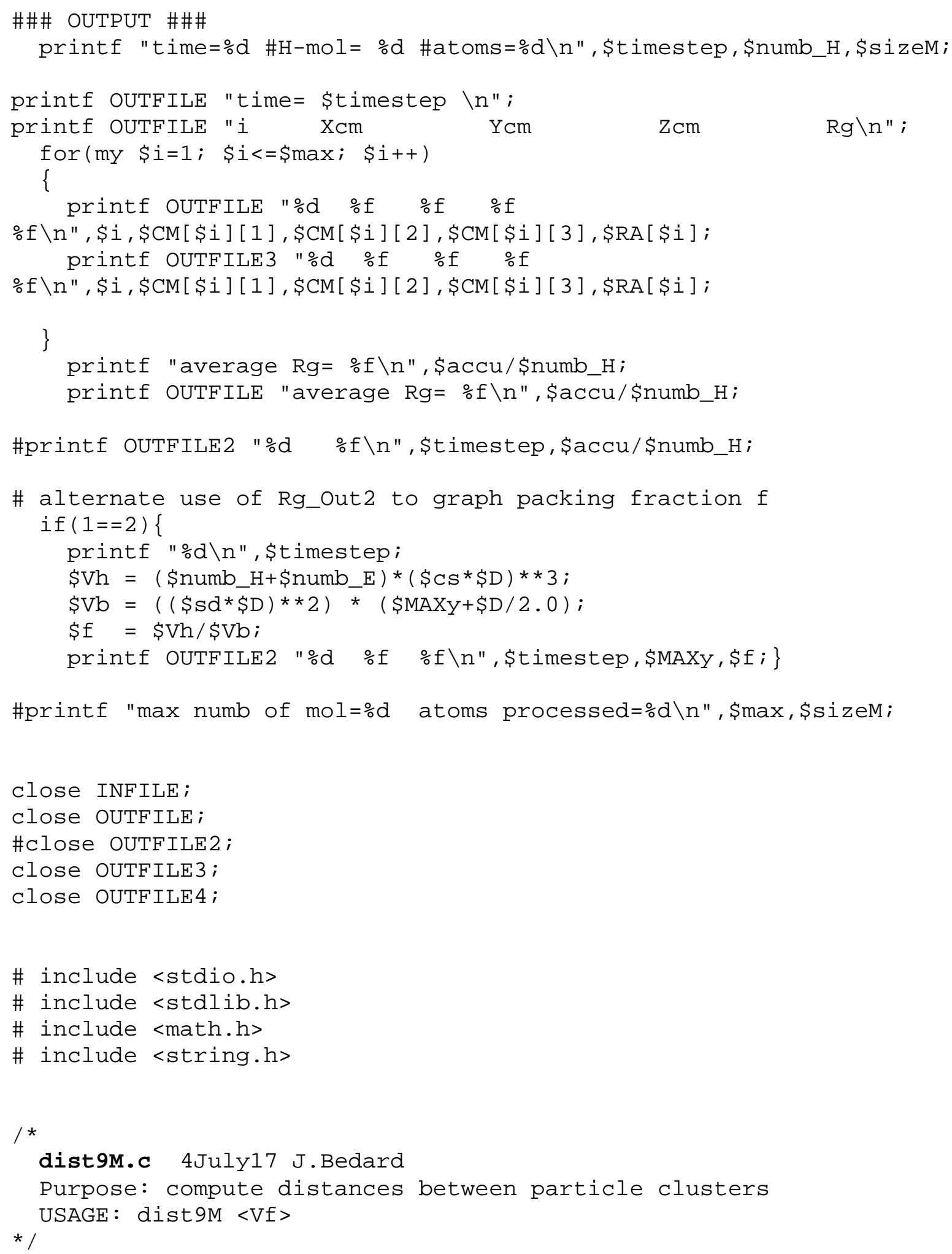




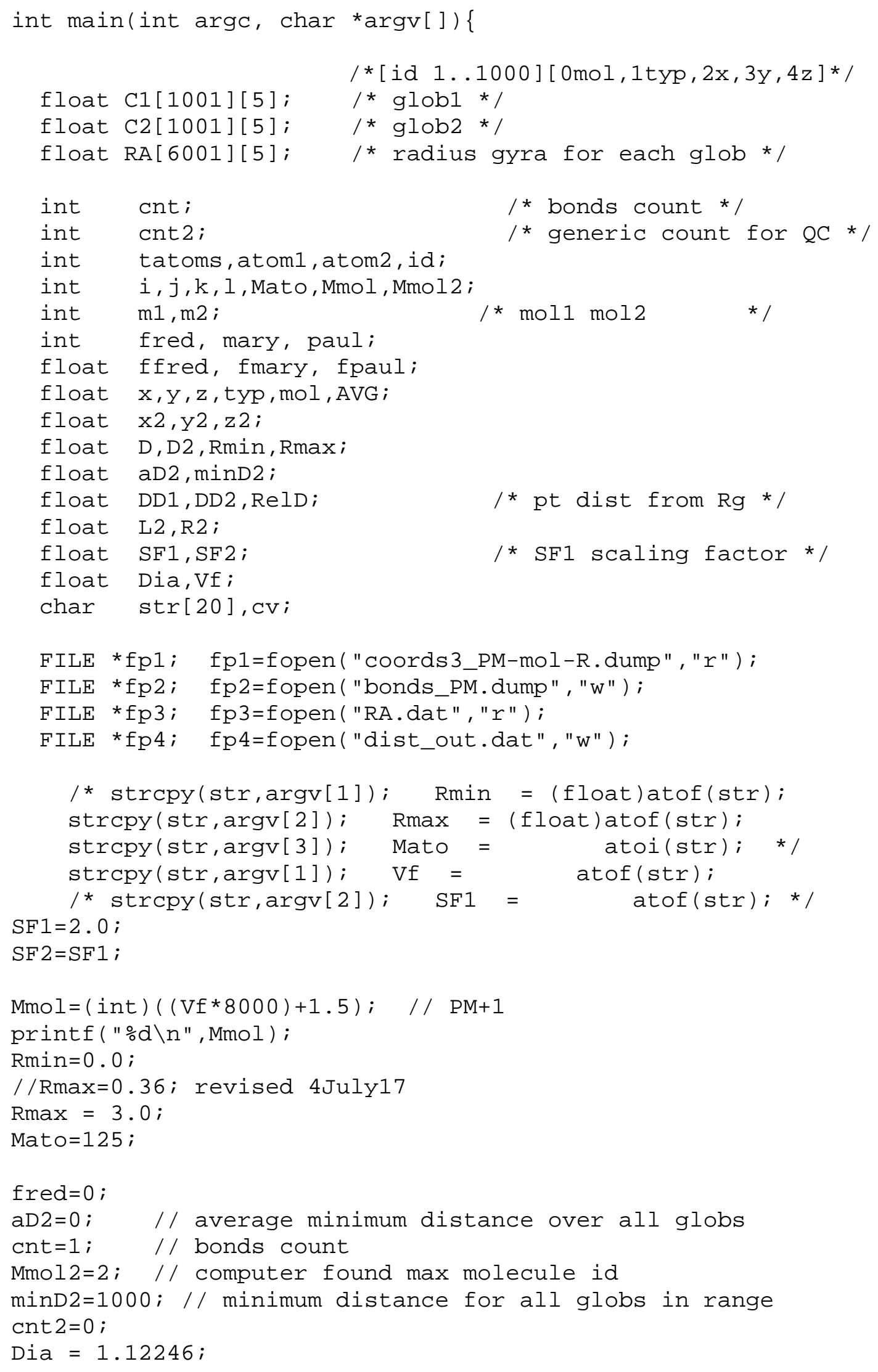




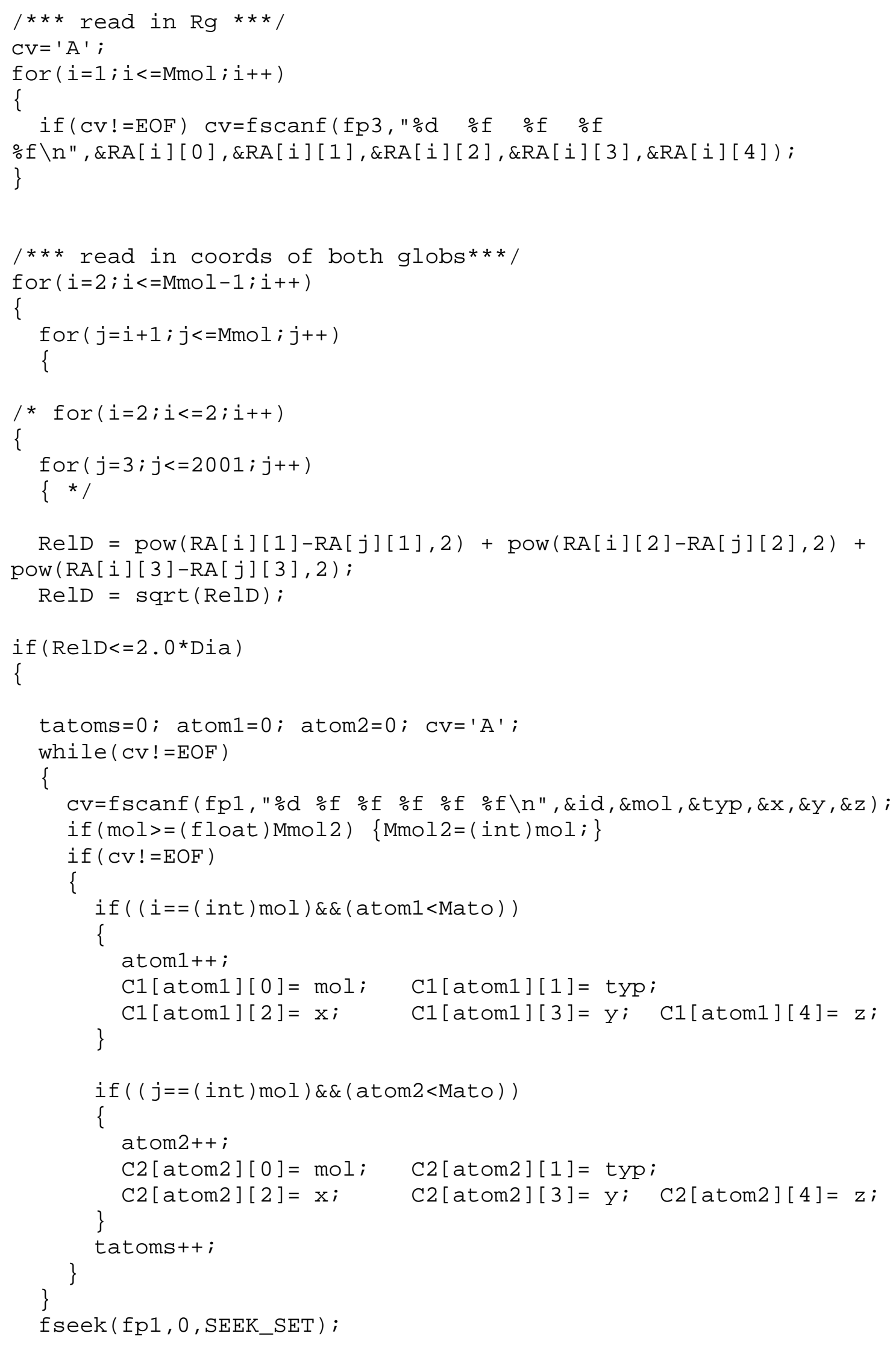


1 * compute minimum dist for all atoms between two globs* D2 $=1000.0$;

for $(\mathrm{k}=1 ; \mathrm{k}<=$ Mato $; \mathrm{k}++)$

\{

for $(1=1 ; 1<=$ Mato $; 1++)$

\{

$\mathrm{m} 1=\mathrm{C} 1[\mathrm{k}][0] ; \quad \mathrm{m} 2=\mathrm{C} 2[1][\Theta] ;$

$\mathrm{DD} 1=\operatorname{pow}(\mathrm{C} 1[\mathrm{k}][2]-\mathrm{RA}[\mathrm{m1}][1], 2)+\operatorname{pow}(\mathrm{C} 1[\mathrm{k}][3]-$

$\mathrm{RA}[\mathrm{m} 1][2], 2)+\operatorname{pow}(\mathrm{C} 1[\mathrm{k}][4]-\mathrm{RA}[\mathrm{m} 1][3], 2)$;

$\mathrm{DD} 2=\operatorname{pow}(\mathrm{C} 2[1][2]-\mathrm{RA}[\mathrm{m} 2][1], 2)+\operatorname{pow}(\mathrm{C} 2[1][3]-$

$\operatorname{RA}[\mathrm{m} 2][2], 2)+\operatorname{pow}(\mathrm{C} 2[1][4]-\operatorname{RA}[\mathrm{m} 2][3], 2)$;

DD1=sqrt (DD1)； $\quad$ DD2=sqrt (DD2)；

if (DD1>SF1*RA[m1][4]) fred++;

if (DD2>SF2*RA[m2][4]) fred ++ ;

if $\left(\left(\mathrm{DD} 1<=\mathrm{SF} 1{ }^{*} \mathrm{RA}[\mathrm{m} 1][4]\right) \& \&\left(\mathrm{DD} 2<=\mathrm{SF} 2{ }^{*} \mathrm{RA}[\mathrm{m} 2][4]\right)\right)$

\{

$\mathrm{x} 2=\operatorname{pow}(\mathrm{C} 1[\mathrm{k}][2]-\mathrm{C} 2[1][2], 2) ;$

$\mathrm{y} 2=\operatorname{pow}(\mathrm{C} 1[\mathrm{k}][3]-\mathrm{C} 2[1][3], 2) ;$

$\mathrm{z2}=\operatorname{pow}(\mathrm{C} 1[\mathrm{k}][4]-\mathrm{C} 2[1][4], 2)$;

$\mathrm{D}=\operatorname{sqrt}(\mathrm{x} 2+\mathrm{y} 2+\mathrm{z} 2)$

if $((C 1[i][\odot] !=C 2[j][\odot]) \& \&(D>=\operatorname{Rmin}) \& \&(D<=\operatorname{Rmax}))$

\}$^{\}}$ $\{$ if $(\mathrm{D}<\mathrm{D} 2) \quad\{\mathrm{L} 2=\mathrm{C} 1[\mathrm{k}][\Theta] ; \mathrm{R} 2=\mathrm{C} 2[1][\Theta] ; \mathrm{D} 2=\mathrm{D} ;\}\}$

$/ /$ if $((\mathrm{C} 1[\mathrm{i}][\Theta] !=\mathrm{C} 2[\mathrm{j}][\Theta]) \& \&(\mathrm{D} 2>=\operatorname{Rmin}) \& \&(\mathrm{D} 2<=\operatorname{Rmax}))$ if $((\mathrm{D} 2>=\mathrm{Rmin}) \& \&(\mathrm{D} 2<=\mathrm{Rmax}))$

\{

fprintf(fp2, "\%d \%4. of \%4. of \%f $\odot . \odot \backslash n "$, cnt, L2, R2, D2) ; \} cnt++; aD2=aD2+D2; if (D2<minD2) \{minD2=D2;\}

\} //for $\mathrm{j}$

\} //endif

if ( $i \% 100==\odot)$ \{printf( $i, j, c n t: \% d \quad \% d \quad \% d \quad D D 1, D D 2: \% f$ $\% f \backslash n ", i, j, c n t, D D 1, D D 2) ;\}$

\} //for i

$\mathrm{SF} 1=($ float $) \mathrm{fred} /\left(\right.$ Mato* $\left.\mathrm{Vf}{ }^{*} 8000.0\right)$;

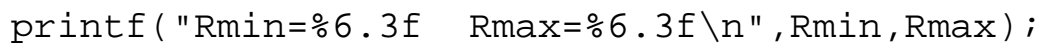

printf("Mmol_id=\%d total bonds=\%d $\backslash n ", M m o l 2$, cnt -1$)$; 
printf("Dmin=\%6.3f Davg=\%6.3f\n", minD2, aD2/(float)(cnt-1));

printf("freds=\%d percent freds=\%6.3f\%\n",fred, SF1*100);

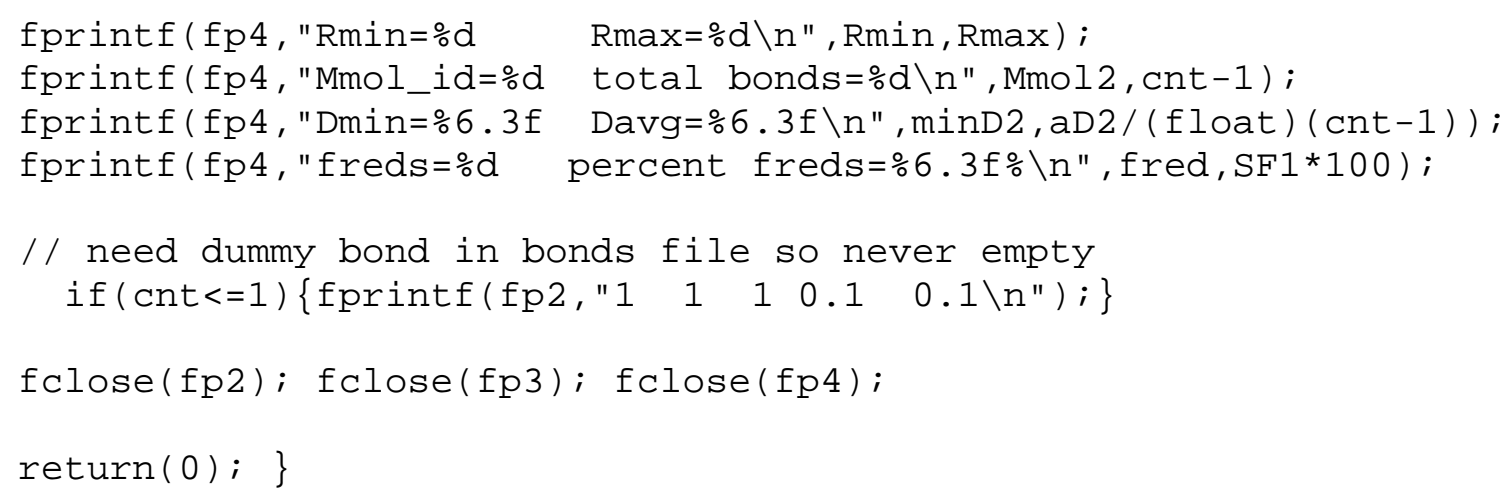




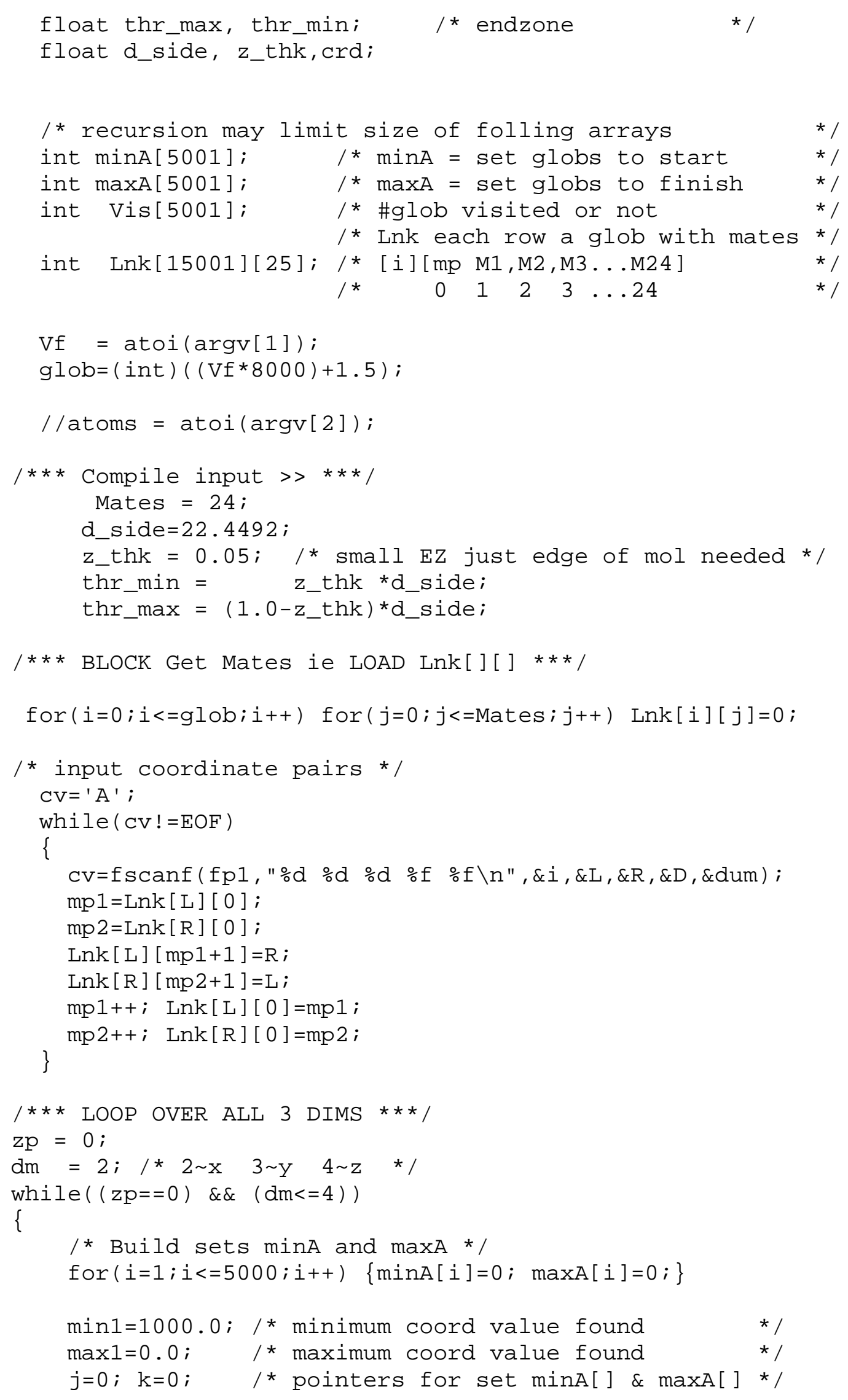




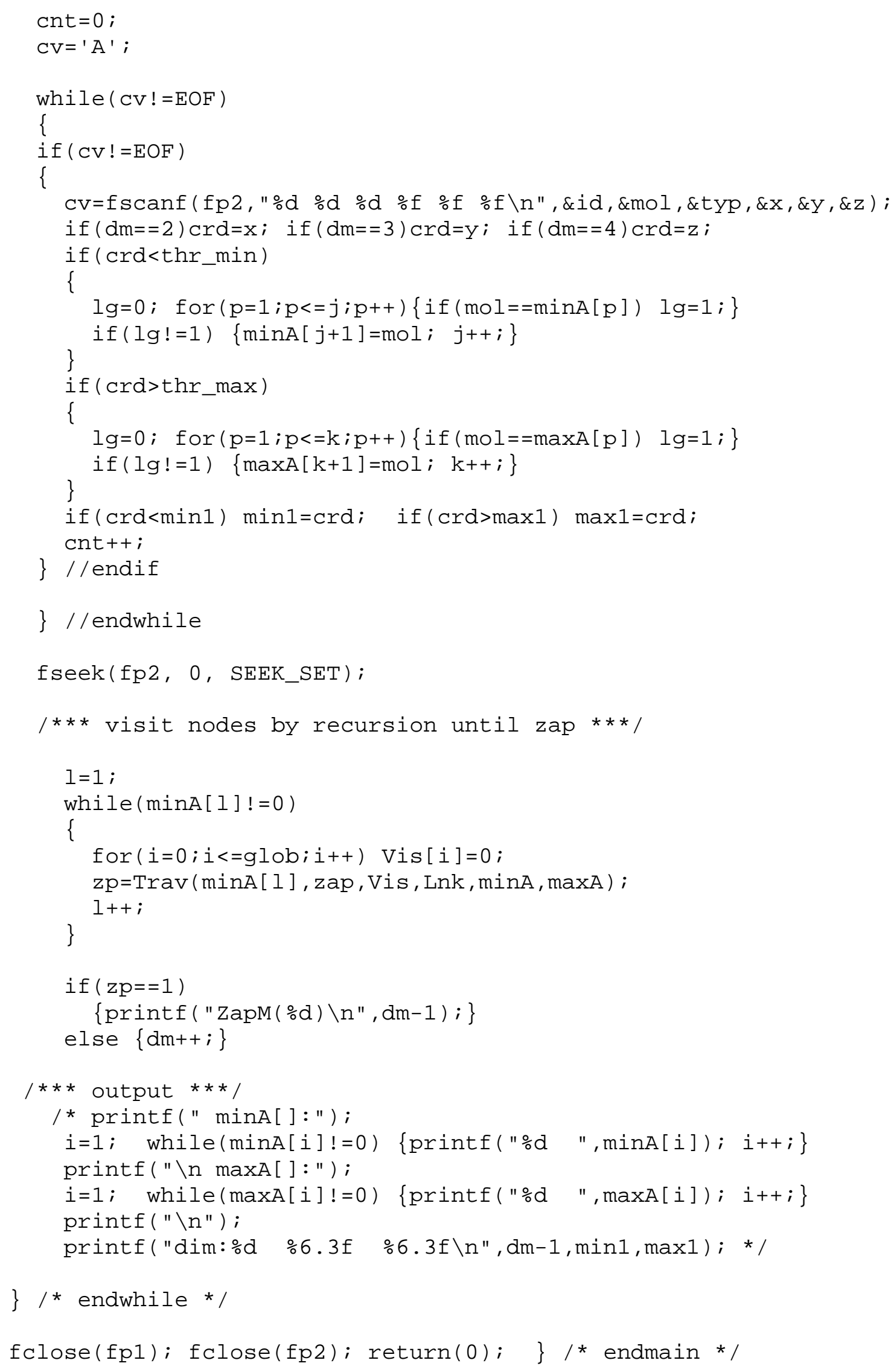




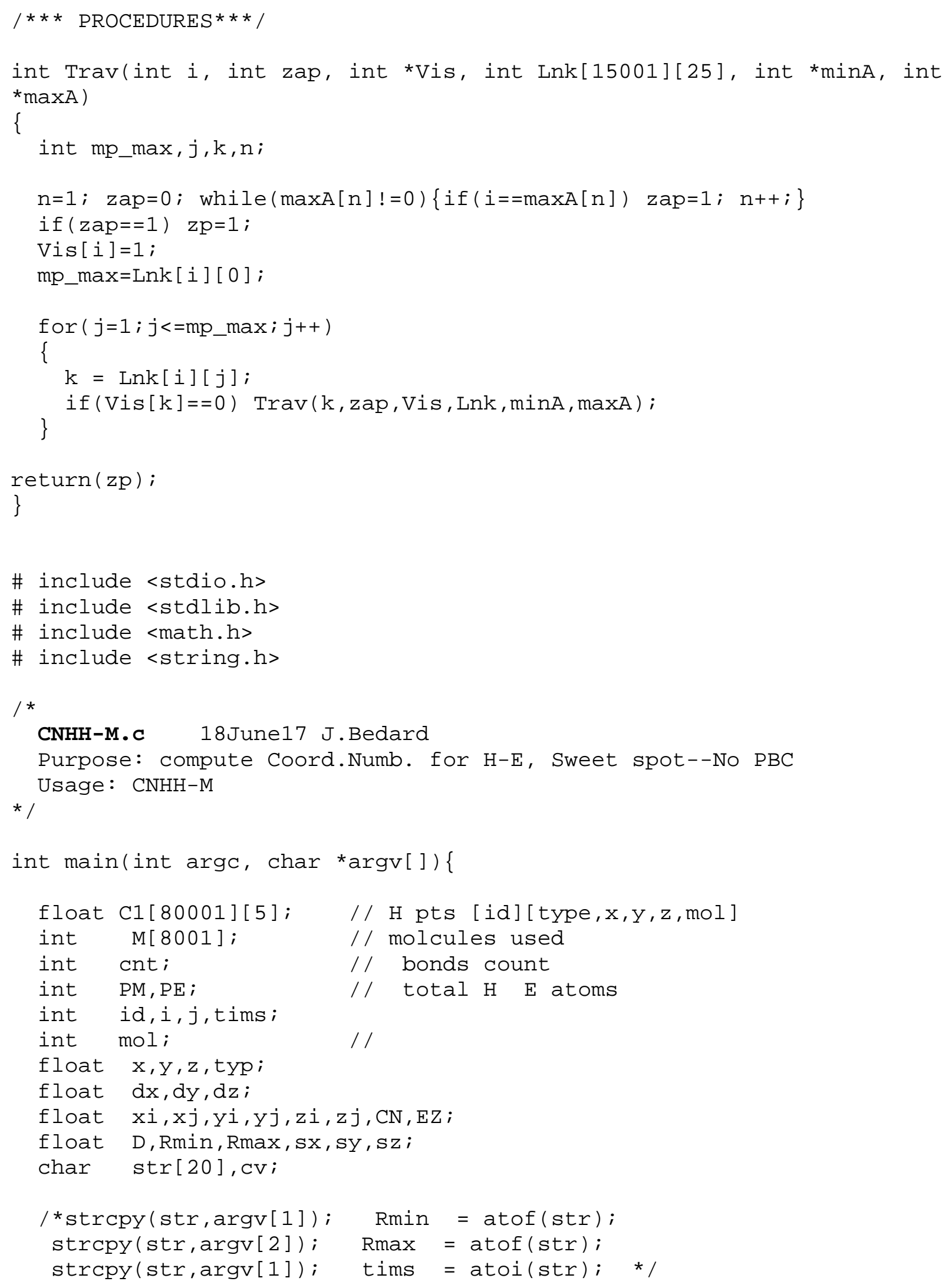




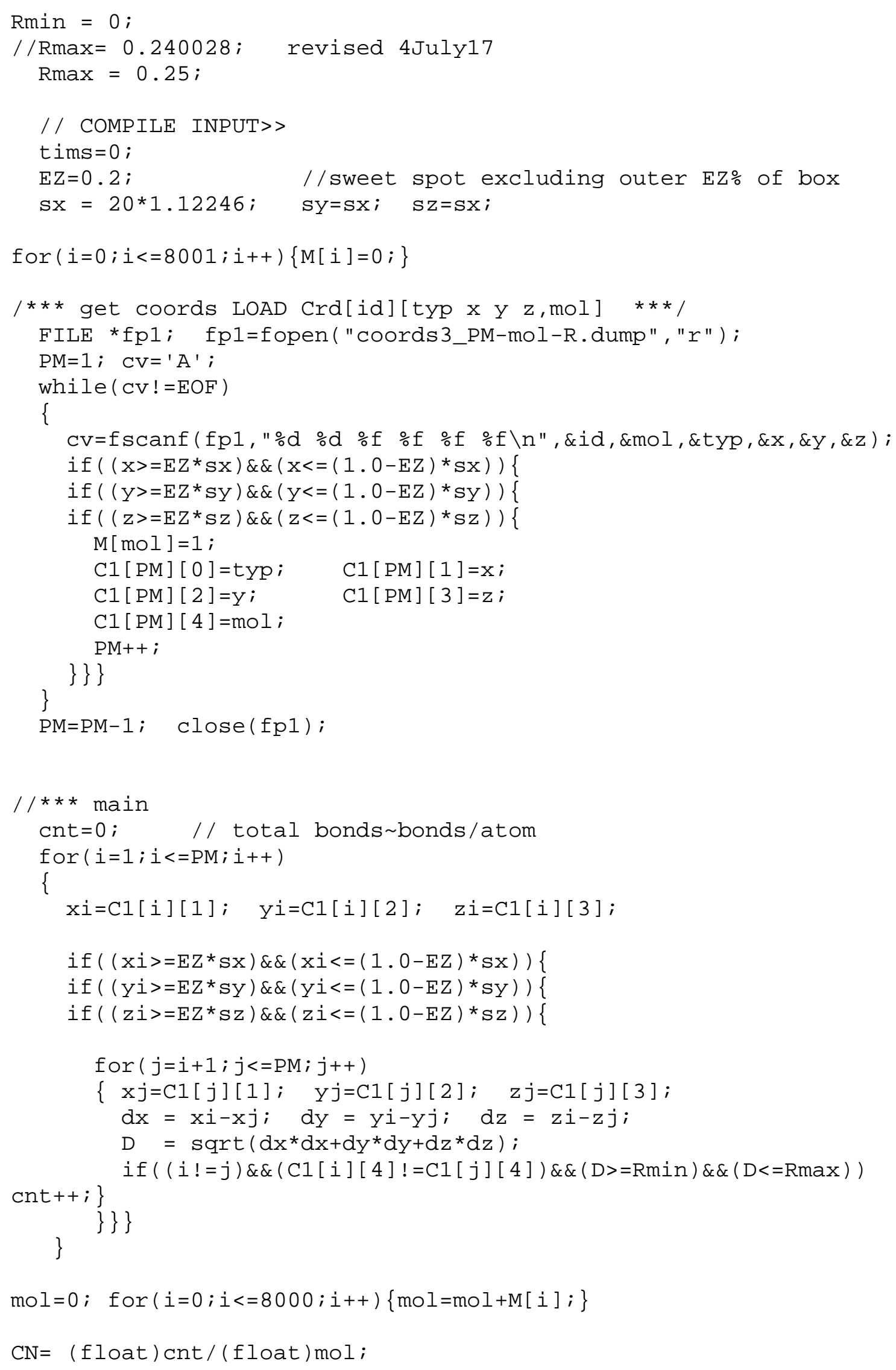


printf("\%d \%6.3f\n", mol, CN);

$\operatorname{return}(0) ;\}$

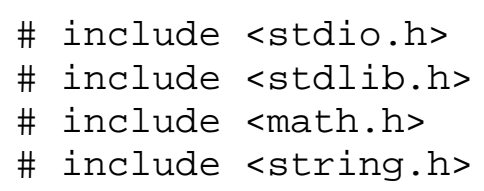




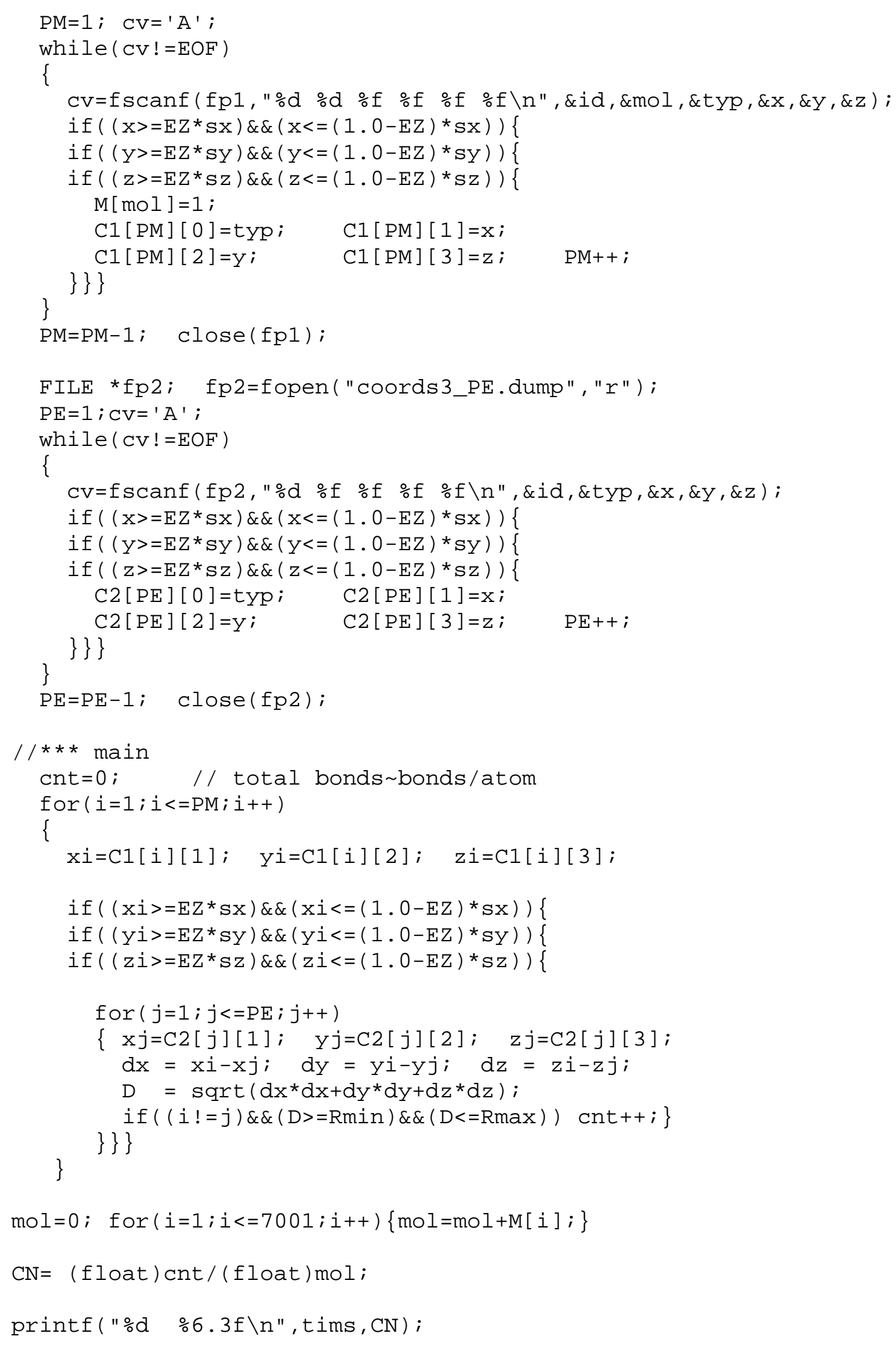


$\operatorname{return}(\odot) ;\}$ 
Appendix C. Lammps scripts

The following script files are selected input scripts used by Lammps (C) for this study. Files need to be adjusted and customized depending on particles size and volume fraction of particles. The scripts presented here are applied for no-affinity (Noa) conditions. To apply scripts for the Affinity (Adh) conditions, extend the sigma parameters as needed. All LAMMPS scripts were run with LAMMPS version compiled May 2016 with following command: lmp < input_file.txt

$\underline{\text { Index of LAMMPS scripts }} \quad \underline{\text { Explanation }} \quad \underline{\text { Page }}$

1. input_MIX.txt $\quad$ Used in MIX stage for all particle sizes 130

2. input_COOL1.txt Used in EQU stage for RH/RE = 1, 2, 3, and for R=all 131

3. input_COOL2.txt Used in GEL stage for RH/RE = 1, 2, 3, and for R=all 132

4. input_EQU1.txt $\quad$ Used in EQU stage for RH/RE = 4 134

5. input_EQU2.txt Used in EQU stage for RH/RE = 4 following EQU1 135

6. input_GEL1.txt Used in GEL stage for RH/RE = 4 136

7. input_GEL2.txt Used in GEL stage for RH/RE = 4 following GEL1 138

8. input_MIX7.txt $\quad$ Used after scripts to mix deformable clusters 139 
LAMMPS script files used in this study

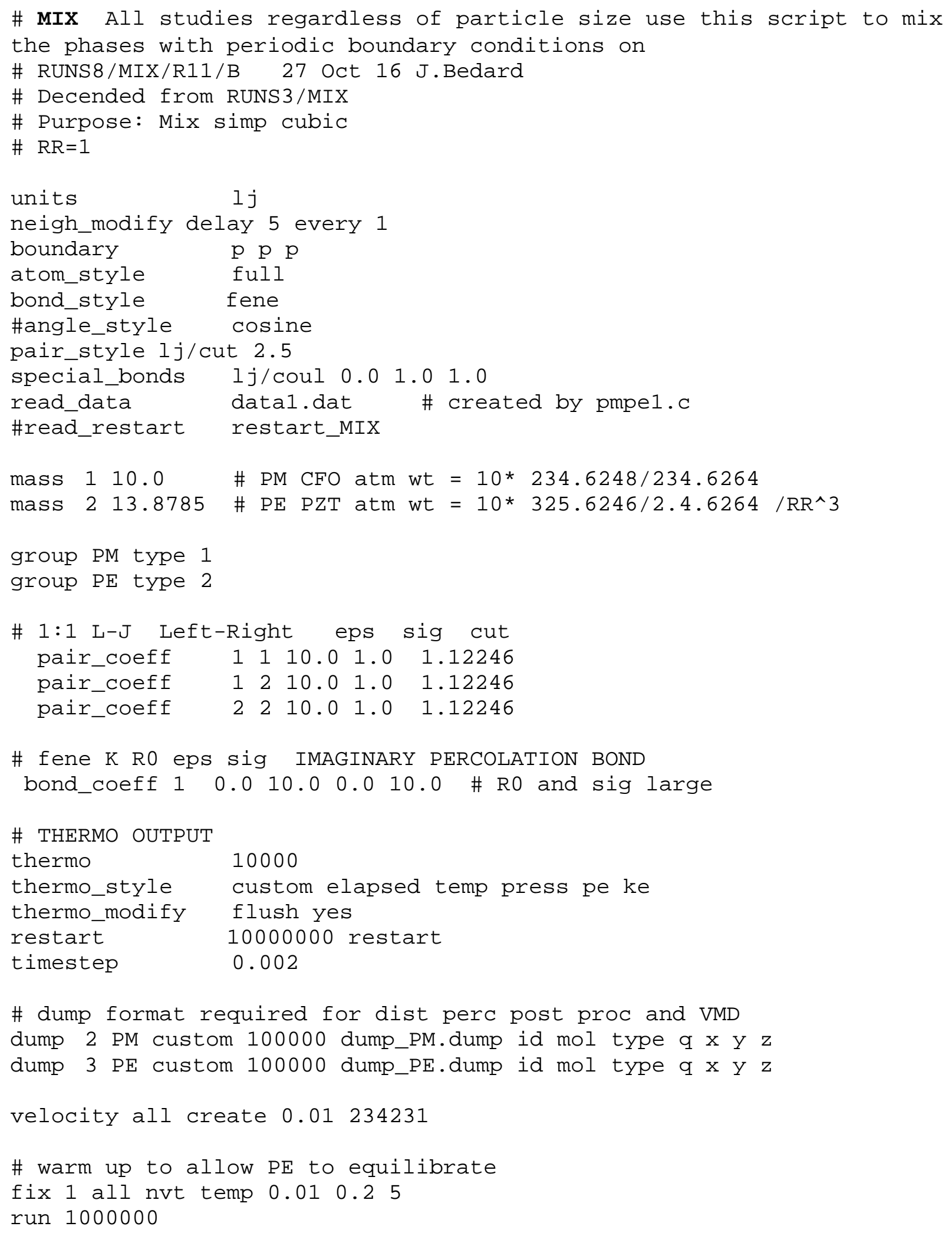




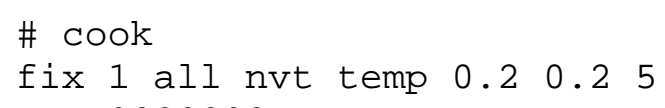

\# dump format required for dist and perc post processing dump 2 PM custom 10000 dump_PM.dump id mol type q x y z dump 3 PE custom 10000 dump_PE.dump id mol type $q \times$ y $z$

\# COMPACT atoms -y dir

\#fix 2 all gravity 0.05 spherical -9090

\#fix 2 all addforce $\odot . \odot-\odot .015$ ๑.๑

fix 3 all wall/reflect xlo EDGE xhi EDGE ylo EDGE yhi EDGE zlo EDGE zhi EDGE

\#fix 3 all wall/gran 2000 NuLL $5 \odot$ NuLL $\odot .5 \odot$ yplane $\odot 11.2$ 


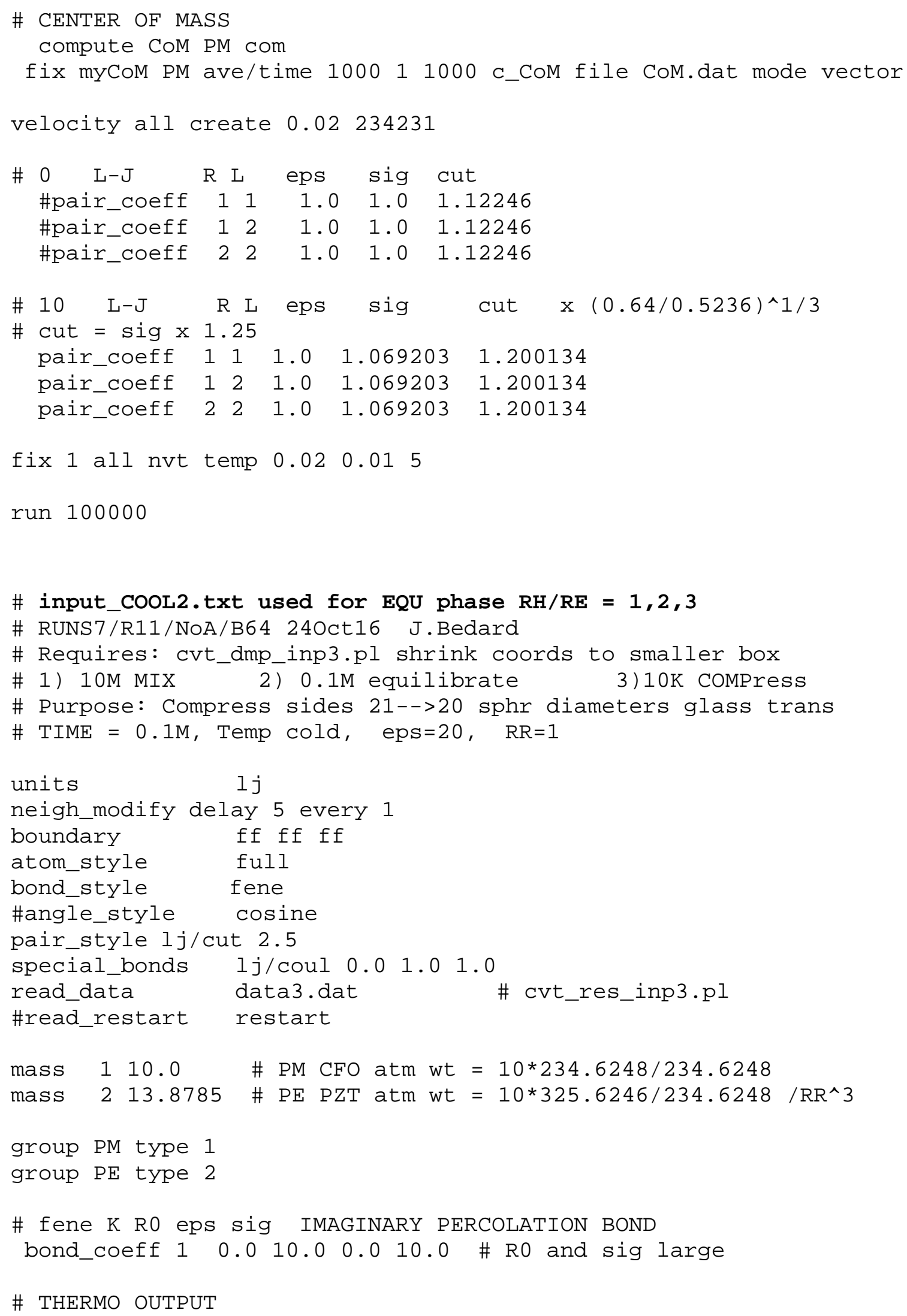




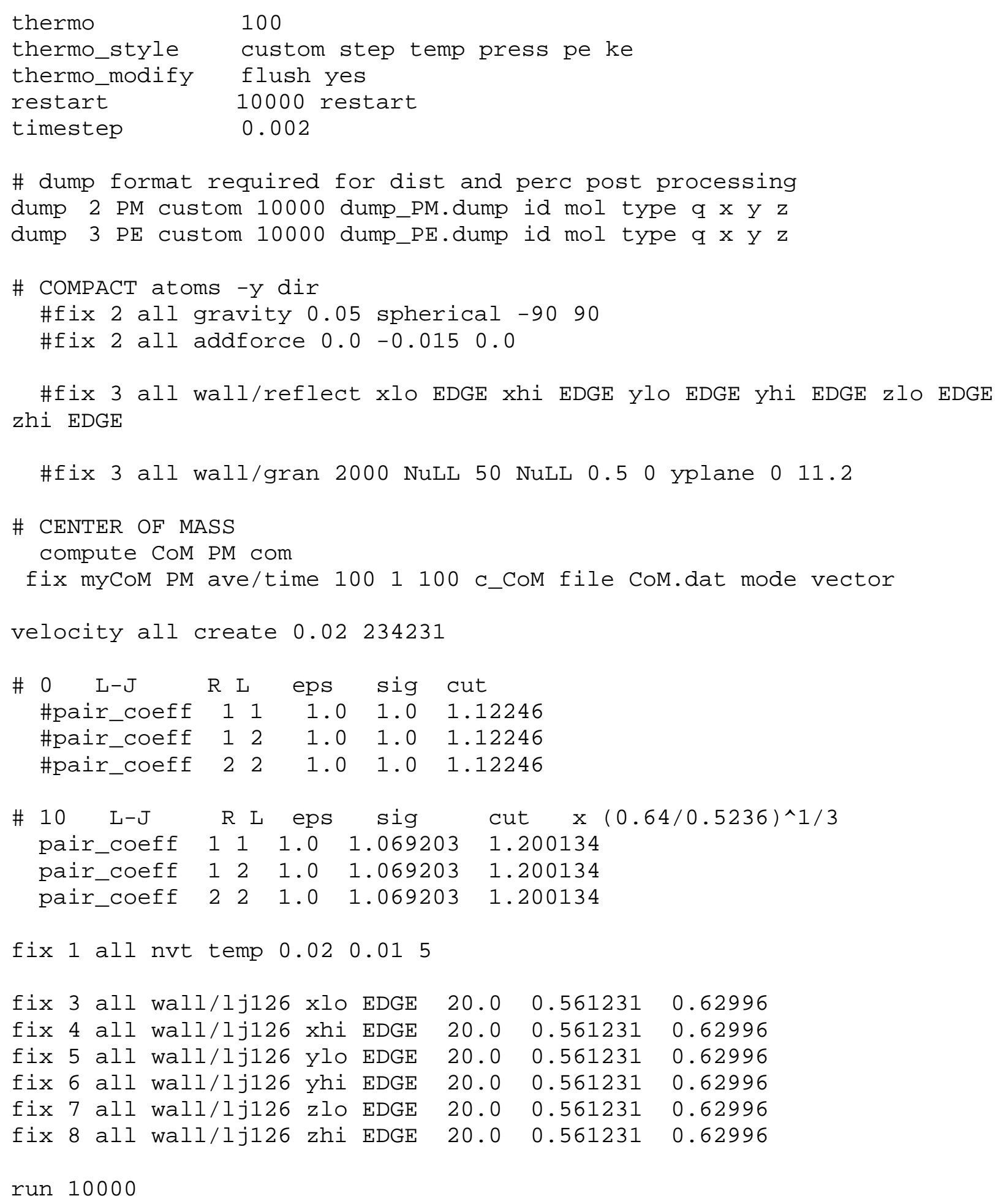

fix 1 all nvt temp 0.020 .015

fix 3 all wall/lj126 xlo EDGE $20.0 \quad 0.561231 \quad 0.62996$

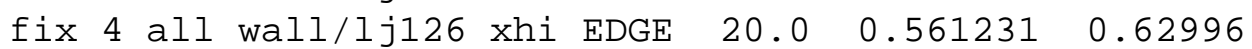

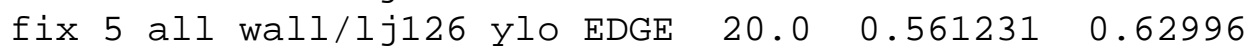

fix 6 all wall/lj126 yhi EDGE 20.๑ $0.561231 \quad 0.62996$

fix 7 all wall/lj126 zlo EDGE $20.0 \quad 0.561231 \quad 0.62996$

fix 8 all wall/lj126 zhi EDGE $20 . \odot \quad 0.561231 \quad 0.62996$

run 10000 


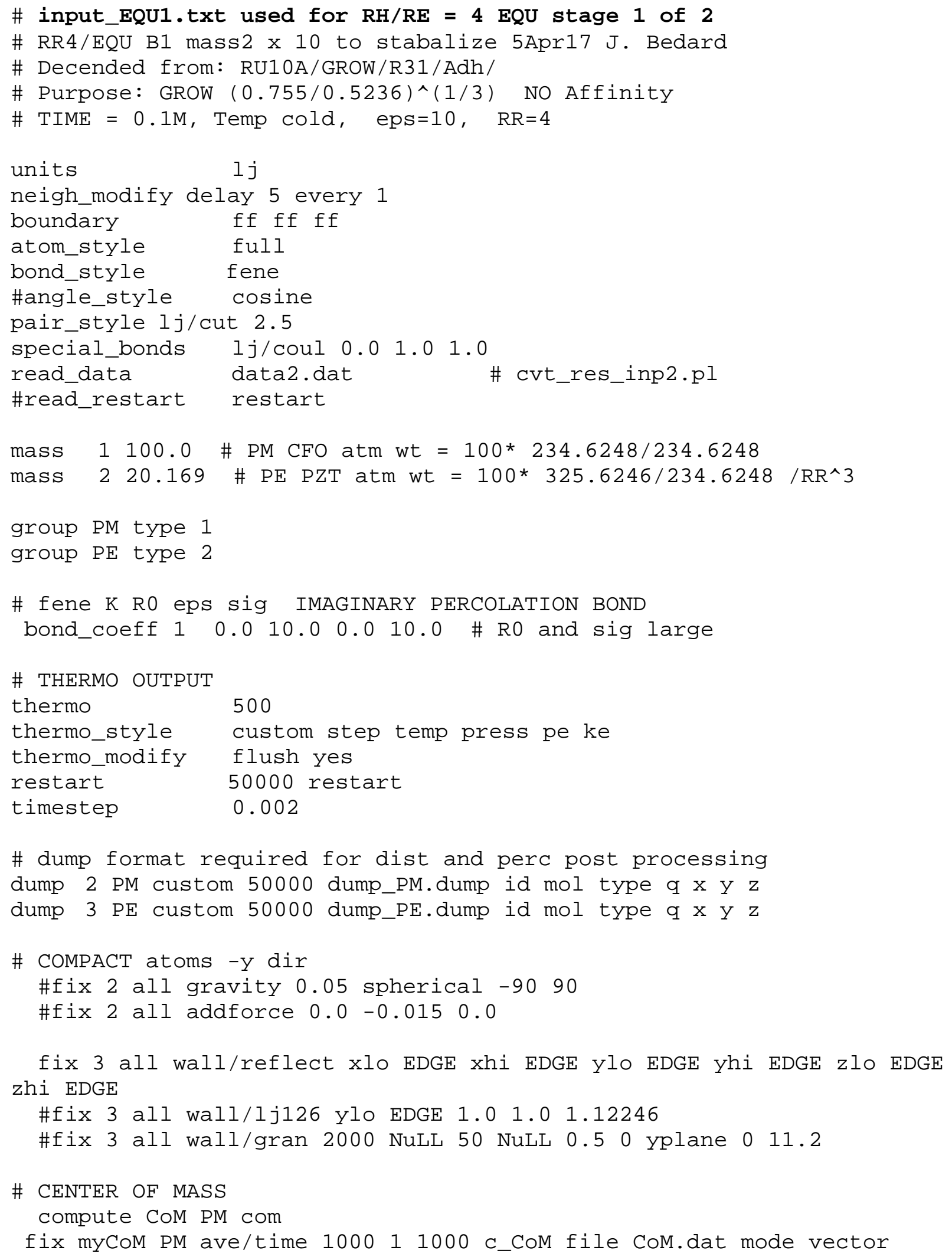




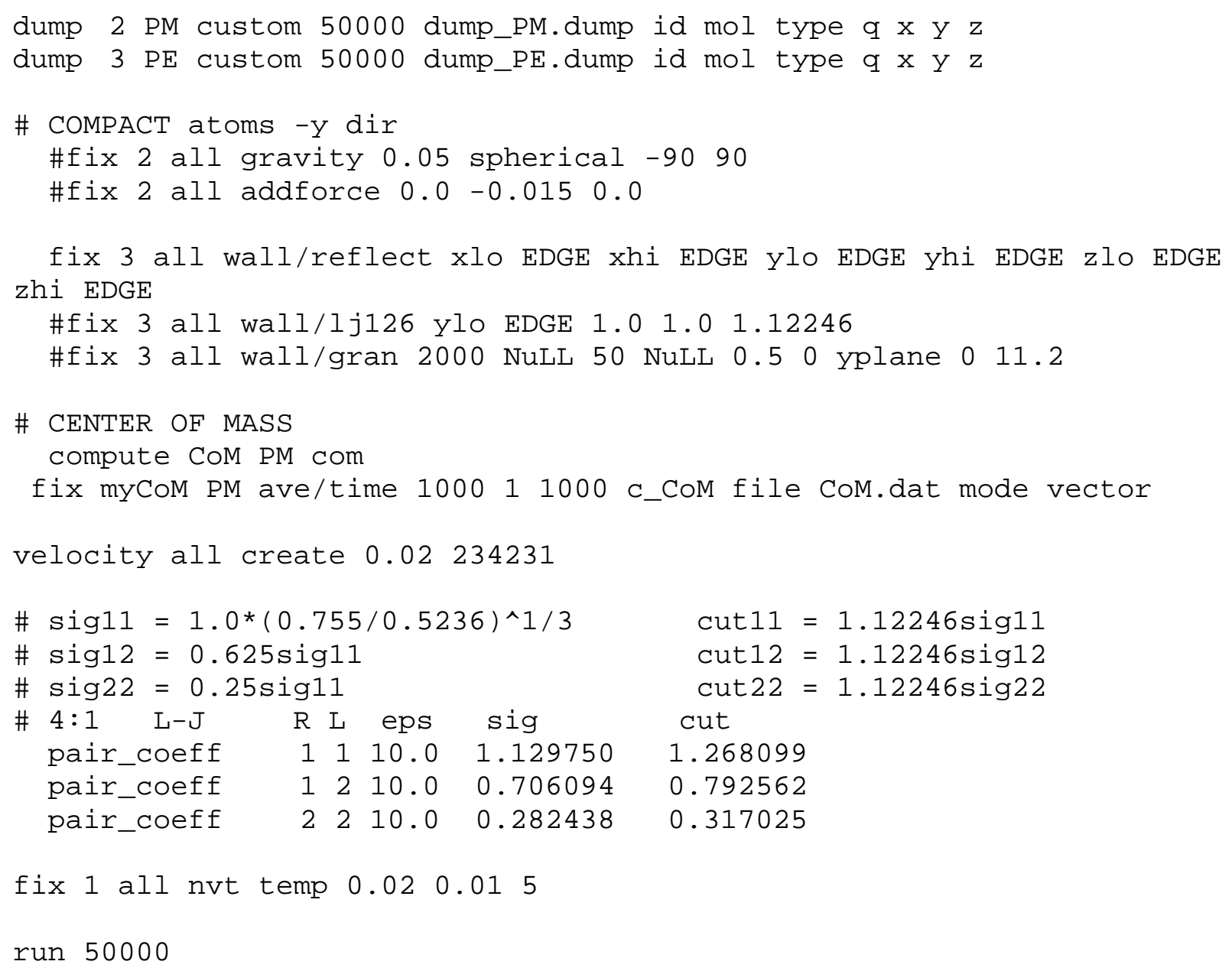




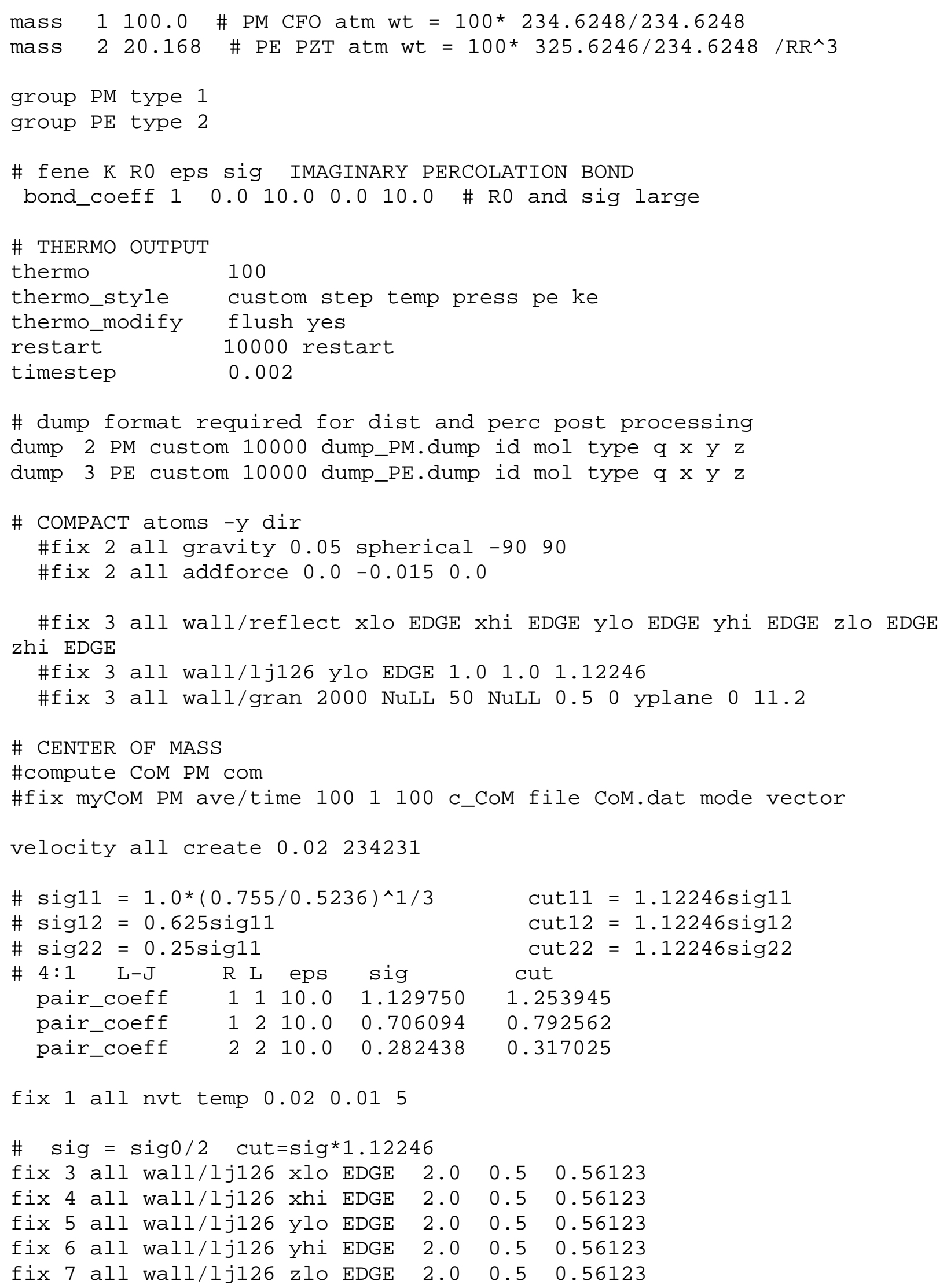

fix 1 all nvt temp 0.020 .015

$\# \quad$ sig $=$ sig®/2 cut=sig*1.12246

fix 3 all wall/lj126 xlo EDGE $2 . \odot \quad 0.5 \quad 0.56123$

fix 4 all wall/lj126 xhi EDGE $2.0 \quad 0.5 \quad 0.56123$

fix 5 all wall/lj126 ylo EDGE $2.0 \quad 0.5 \quad 0.56123$

fix 6 all wall/lj126 yhi EDGE $2.0 \quad 0.5 \quad 0.56123$

$\begin{array}{llllll}\text { fix } 7 \text { all wall/lj126 zlo EDGE } 2 . \odot & 0.5 & 0.56123\end{array}$ 


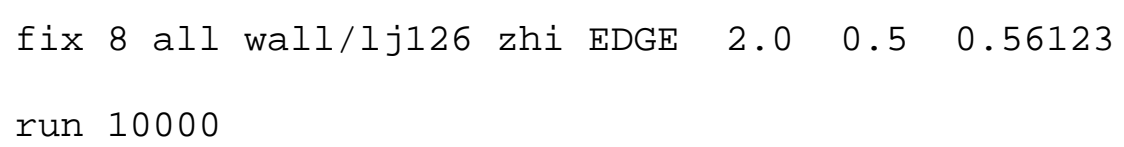




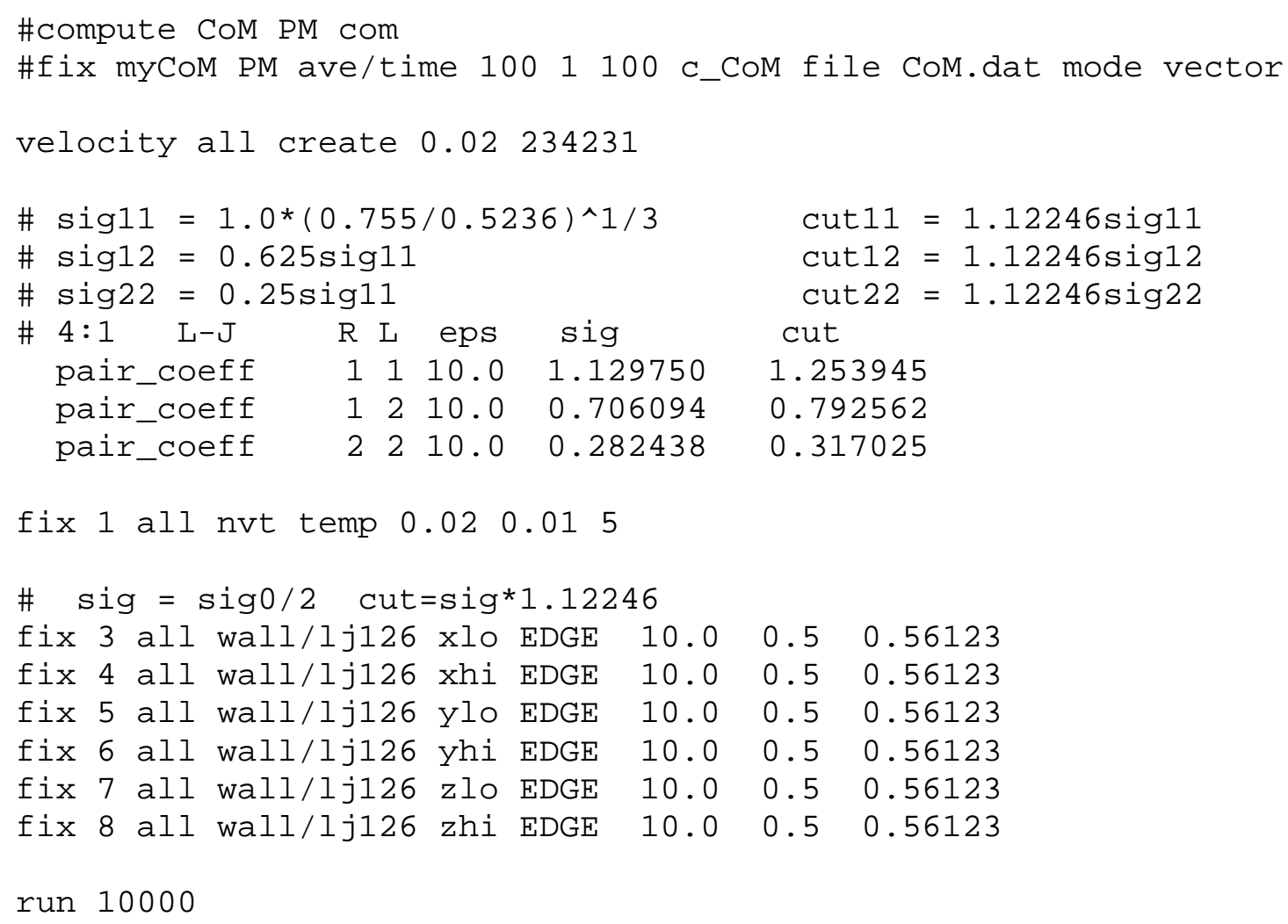




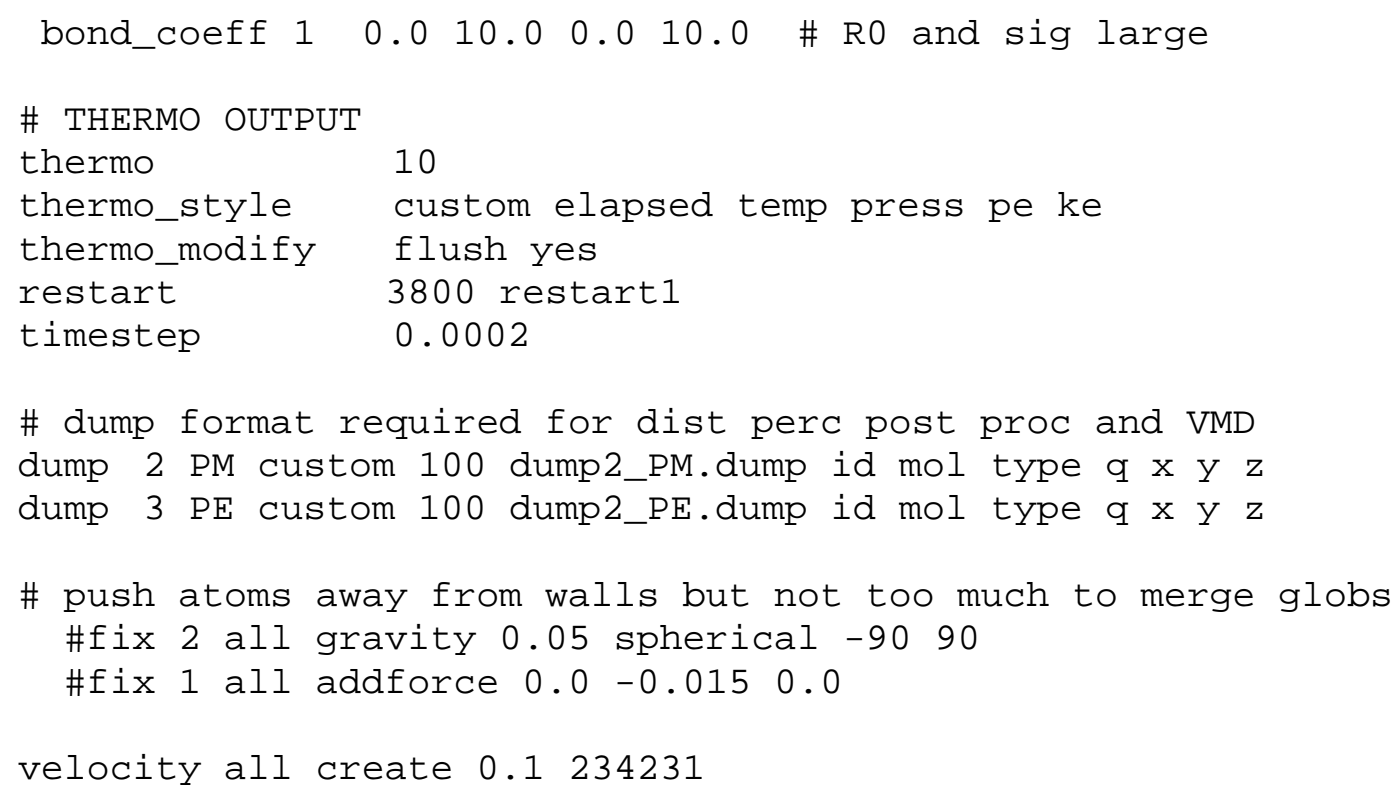


fix 6 all wall/lj126 yhi EDGE $2 . \odot \quad 0.12 \quad 0.134695$

fix 7 all wall/lj126 zlo EDGE $2.0 \quad 0.120 .134695$

fix 8 all wall/lj126 zhi EDGE $2.0 \quad 0.12 \quad 0.134695$

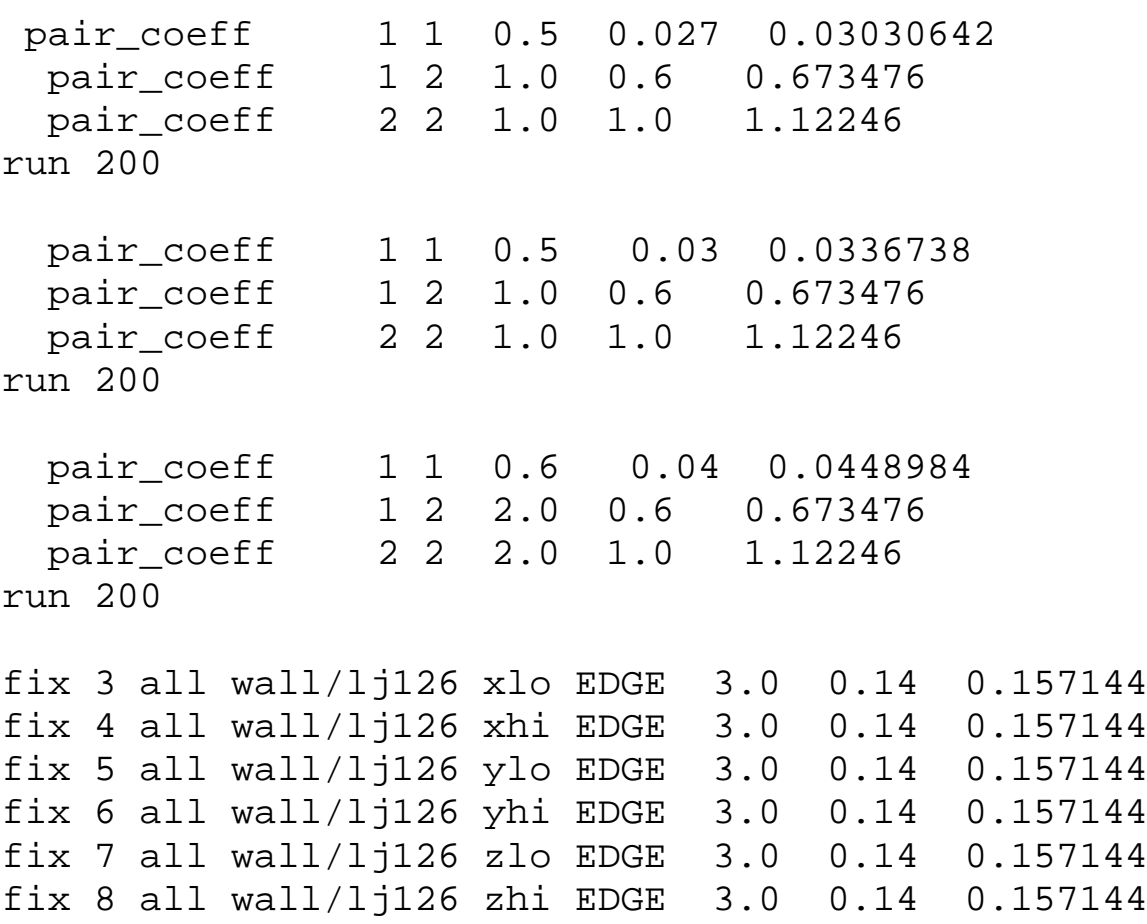
pair_coeff
$\begin{array}{lll}1 & 1 & 0.6\end{array}$
0.045
0.050510
pair_coeff
$\begin{array}{llll}1 & 2 & 2.0 & 0.6\end{array}$
$\odot .673476$
pair_coeff
$\begin{array}{llll}2 & 2 & 2.0 & 1.0\end{array}$
1.12246

run 200
pair_coeff
$\begin{array}{lll}1 & 1 & 0.6\end{array}$
0.05
0.056123
pair_coeff
122.0
0.6
0.673476
pair_coeff
222.0
1.0
1.12246

run 200
pair_coeff
$\begin{array}{lll}1 & 1 & 0.7\end{array}$
0.06
0.067348
pair_coeff
121.0
0.6
0.673476
pair_coeff
221.0
1.01 .12246

run $20 \overline{0}$

fix 3 all wall/lj126 xlo EDGE $4.0 \quad 0.2$ 0.224492

fix 4 all wall/lj126 xhi EDGE $4.0 \quad 0.2$ ०.224492

fix 5 all wall/lj126 ylo EDGE $4.0 \quad 0.2$ 0.224492

fix 6 all wall/lj126 yhi EDGE $4.0 \quad 0.2$ ०.224492

fix 7 all wall/lj126 zlo EDGE $4.0 \quad 0.2$ 0.224492

fix 8 all wall/lj126 zhi EDGE $4.0 \quad 0.2 \quad 0.224492$
pair_coeff
$\begin{array}{lll}1 & 1 & 0.7\end{array}$
$\odot . \odot 7$
$\odot .0785572$
pair_coeff
$\begin{array}{lll}1 & 2 & 3.0\end{array}$
$0.6 \quad 0.673476$
pair_coeff
223.0
$1.0 \quad 1.12246$ 
run 100

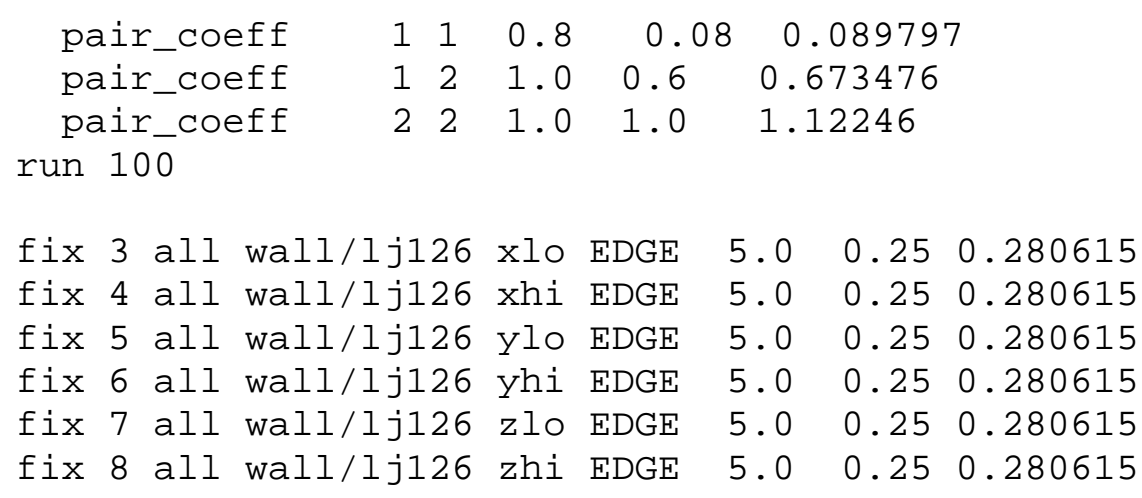

\begin{tabular}{|c|c|c|c|c|}
\hline pair_coeff & 11 & 0.8 & 0.09 & 0.101214 \\
\hline pair_coeff & 12 & 4.0 & 0.6 & $\odot .673476$ \\
\hline pair_coeff & 22 & 4.0 & 1.0 & 1.12246 \\
\hline
\end{tabular}

$\begin{array}{lllllll}\text { pair_coeff } & 1 & 1 & 0.9 & 0.1 & 0.112246 \\ \text { pair_coeff } & 1 & 2 & 1.0 & 0.6 & 0.673476 & \\ \text { pair_coeff } & 2 & 2 & 1.0 & 1.0 & 1.12246 & \\ \text { run } 10 \odot & & & & & & \\ & & & & & & \\ \text { fix } 3 \text { all wall/lj126 } & \text { xlo EDGE } & 6 . \odot & 0.3 & 0.336738 \\ \text { fix } 4 \text { all wall/lj126 xhi EDGE } & 6 . \odot & 0.3 & 0.336738 \\ \text { fix } 5 \text { all wall/lj126 ylo EDGE } & 6 . \odot & 0.3 & 0.336738 \\ \text { fix } 6 \text { all wall/lj126 yhi EDGE } & 6 . \odot & 0.3 & 0.336738 \\ \text { fix } 7 \text { all wall/lj126 } & \text { zlo EDGE } & 6 . \odot & 0.3 & 0.336738 \\ \text { fix } 8 \text { all wall/lj126 zhi EDGE } & 6 . \odot & 0.3 & 0.336738\end{array}$

$\begin{array}{lrlllll}\text { pair_coeff } & 1 & 1 & 0.9 & 0.11 & 0.123471 \\ \text { pair_coeff } & 1 & 2 & 5.0 & 0.6 & 0.673476 & \\ \text { pair_coeff } & 2 & 2 & 5.0 & 1.0 & 1.12246 & \\ \text { run } 10 \odot & & & & & \\ & & & & & \\ \text { pair_coeff } & 1 & 1 & 1.0 & 0.12 & 0.134695 & \\ \text { pair_coeff } & 1 & 2 & 6.0 & 0.6 & 0.673476 & \\ \text { pair_coeff } & 2 & 2 & 6.0 & 1.0 & 1.12246 & \\ \text { run } 10 \odot & & & & & & \\ \text { fix } 3 \text { all wall/lj126 } & \text { xlo EDGE } & 7.0 & 0.35 & 0.392861 \\ \text { fix } 4 \text { all wall/lj126 } & \text { xhi EDGE } & 7.0 & 0.35 & 0.392861 \\ \text { fix } 5 \text { all wall/lj126 ylo EDGE } & 7.0 & 0.35 & 0.392861 \\ \text { fix } 6 \text { all wall/lj126 yhi EDGE } & 7.0 & 0.35 & 0.392861 \\ \text { fix } 7 \text { all wall/lj126 } & \text { zlo EDGE } & 7.0 & 0.35 & 0.392861 \\ \text { fix } 8 \text { all wall/lj126 } & \text { zhi EDGE } & 7.0 & 0.35 & 0.392861\end{array}$

$\begin{array}{llllll}\text { pair_coeff } & 1 & 1 & 1.0 & 0.13 & 0.14592 \\ \text { pair_coeff } & 1 & 2 & 6.0 & 0.6 & 0.673476 \\ \text { pair_coeff } & 22 & 6.0 & 1.0 & 1.12246\end{array}$

run 100 


\begin{tabular}{|c|c|c|c|c|}
\hline pair_coeff & 11 & 1.0 & 0.14 & $\odot .157144$ \\
\hline pair_coeff & 12 & 7.0 & 0.6 & 0.673476 \\
\hline pair_coeff & 22 & 7.0 & 1.0 & 1.12246 \\
\hline
\end{tabular}

fix 3 all wall/lj126 xlo EDGE fix 4 all wall/lj126 xhi EDGE fix 5 all wall/lj126 ylo EDGE fix 6 all wall/lj126 yhi EDGE fix 7 all wall/lj126 zlo EDGE fix 8 all wall/lj126 zhi EDGE

$\begin{array}{lll}7.5 & 0.4 & 0.448984 \\ 7.5 & 0.4 & 0.448984 \\ 7.5 & 0.4 & 0.448984 \\ 7.5 & 0.4 & 0.448984 \\ 7.5 & 0.4 & 0.448984 \\ 7.5 & 0.4 & 0.448984\end{array}$

\begin{tabular}{|c|c|c|c|c|}
\hline pair_coeff & 11 & 1.0 & 0.15 & 0.168369 \\
\hline pair_coeff & 12 & 7.0 & 0.6 & 0.673476 \\
\hline pair_coeff & 22 & 7.0 & 1.0 & 1.12246 \\
\hline
\end{tabular}

run 100

\begin{tabular}{|c|c|c|c|c|}
\hline pair_coeff & 11 & 1.0 & 0.16 & 0.179594 \\
\hline pair_coeff & 12 & 8.0 & 0.6 & 0.673476 \\
\hline pair_coeff & 22 & 8.0 & 1.0 & 1.12246 \\
\hline
\end{tabular}

$\begin{array}{lllllll}\text { fix } 3 \text { all wall/lj126 xlo EDGE } & 8 . \odot & 0.45 & 0.505107 \\ \text { fix } 4 \text { all wall/lj126 } & \text { xhi EDGE } & 8 . \odot & 0.45 & 0.505107 \\ \text { fix } 5 \text { all wall/lj126 ylo EDGE } & 8 . \odot & 0.45 & 0.505107 \\ \text { fix } 6 \text { all wall/lj126 yhi EDGE } & 8 . \odot & 0.45 & 0.505107 \\ \text { fix } 7 \text { all wall/lj126 } & \text { zlo EDGE } & 8 . \odot & 0.45 & 0.505107 \\ \text { fix } 8 \text { all wall/lj126 zhi EDGE } & 8 . \odot & 0.45 & 0.505107\end{array}$

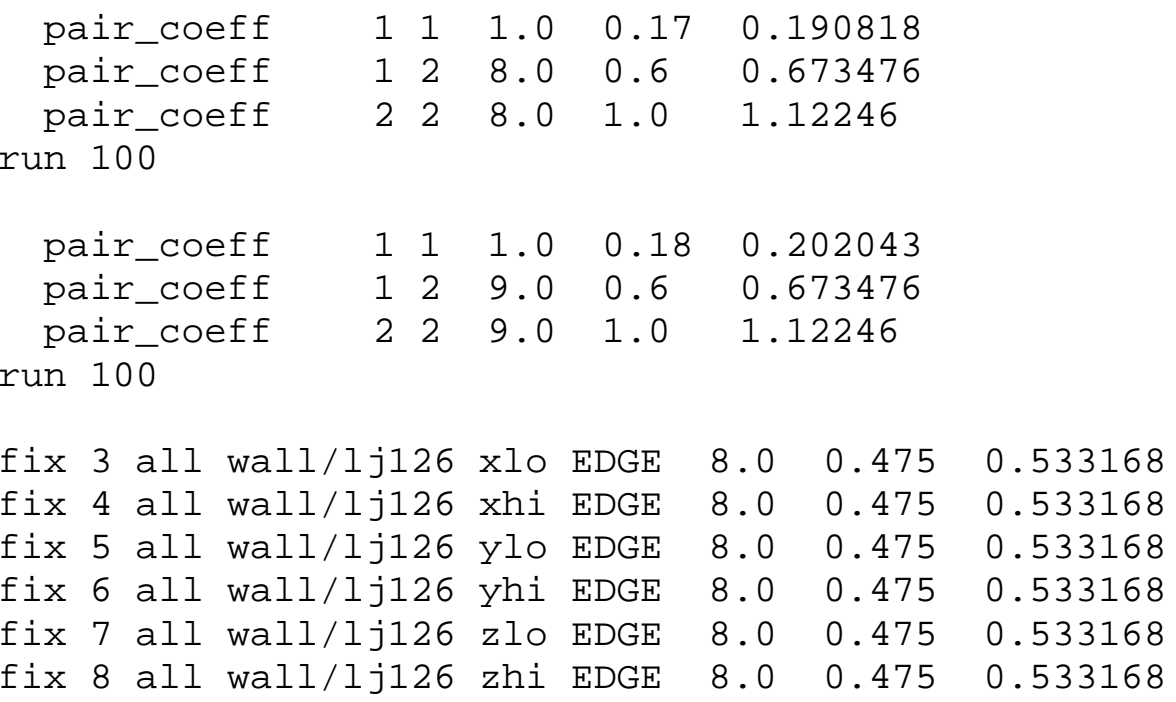

$\begin{array}{llllll}\text { pair_coeff } & 1 & 1 & 1.0 & 0.19 & 0.213267 \\ \text { pair_coeff } & 1 & 2 & 9.0 & 0.6 & 0.673476 \\ \text { pair_coeff } & 2 & 2 & 9.0 & 1.0 & 1.12246\end{array}$


$\begin{array}{lllllll}\text { fix } 3 \text { all wall/lj126 xlo EDGE } & 10 . \odot & 0.5 & 0.56123 \\ \text { fix } 4 \text { all wall/lj126 } & \text { xhi EDGE } & 10 . \odot & 0.5 & 0.56123 \\ \text { fix } 5 \text { all wall/lj126 ylo EDGE } & 10 . \odot & 0.5 & 0.56123 \\ \text { fix } 6 \text { all wall/lj126 yhi EDGE } & 10 . \odot & 0.5 & 0.56123 \\ \text { fix } 7 \text { all wall/lj126 zlo EDGE } & 10 . \odot & 0.5 & 0.56123 \\ \text { fix } 8 \text { all wall/lj126 zhi EDGE } & 10 . \odot & 0.5 & 0.56123\end{array}$

$\begin{array}{lllllll}\text { pair_coeff } & 1 & 1 & 1.0 & 0.2 & 0.224492 & \\ \text { pair_coeff } & 1 & 2 & 10.0 & 0.6 & 0.673476 & \\ \text { pair_coeff } & 2 & 2 & 10.0 & 1.0 & 1.12246 & \\ \text { run } 100 & & & & & & \\ \text { fix } 3 \text { all wall/lj126 } & \text { xlo EDGE } & 12.0 & 0.5 & 0.56123 \\ \text { fix } 4 \text { all wall/lj126 xhi EDGE } & 12.0 & 0.5 & 0.56123 \\ \text { fix } 5 \text { all wall/lj126 ylo EDGE } & 12.0 & 0.5 & 0.56123 \\ \text { fix } 6 \text { all wall/lj126 yhi EDGE } & 12.0 & 0.5 & 0.56123 \\ \text { fix } 7 \text { all wall/lj126 } & \text { zlo EDGE } & 12.0 & 0.5 & 0.56123 \\ \text { fix } 8 \text { all wall/lj126 zhi EDGE } & 12.0 & 0.5 & 0.56123\end{array}$
\# $10 \mathrm{~L}-\mathrm{J} \quad \mathrm{R} \mathrm{L}$ eps sig cut $\times(0.64 / 0.5236)^{\wedge} 1 / 3$
$\begin{array}{lllll}\text { pair_coeff } & 11 & 1.0 & 0.213841 & 0.400275\end{array}$
$\begin{array}{lllll}\text { pair_coeff } & 12 & 10.0 & 0.641522 & 0.720083\end{array}$

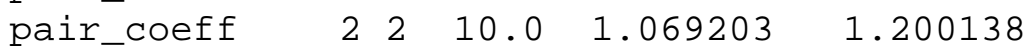
run 100

$\begin{array}{llllllll}\text { fix } 3 \text { all wall/lj126 } & \text { xlo EDGE } & 14.0 & 0.5 & 0.56123 \\ \text { fix } 4 \text { all wall/lj126 } & \text { xhi } \text { EDGE } & 14.0 & 0.5 & 0.56123 \\ \text { fix } 5 \text { all wall/lj126 ylo EDGE } & 14.0 & 0.5 & 0.56123 \\ \text { fix } 6 \text { all wall/lj126 yhi EDGE } & 14.0 & 0.5 & 0.56123 \\ \text { fix } 7 \text { all wall/lj126 } & \text { zlo EDGE } & 14.0 & 0.5 & 0.56123 \\ \text { fix } 8 \text { all wall/lj126 zhi EDGE } & 14.0 & 0.5 & 0.56123\end{array}$

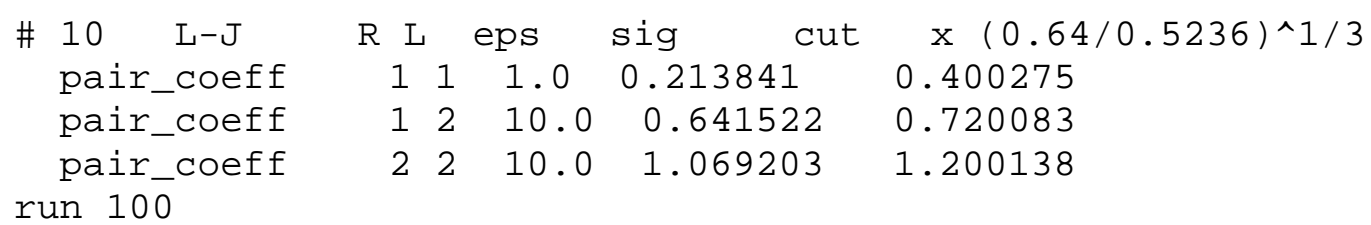

fix 3 all wall/lj126 xlo EDGE $16.0 \quad 0.5 \quad 0.56123$

fix 4 all wall/lj126 xhi EDGE $16.0 \quad 0.5 \quad 0.56123$

fix 5 all wall/lj126 ylo EDGE $16 . \odot \quad 0.5 \quad 0.56123$

fix 6 all wall/lj126 yhi EDGE $16 . \odot \quad 0.5 \quad 0.56123$

fix 7 all wall/lj126 zlo EDGE $16.0 \quad 0.5 \quad 0.56123$

fix 8 all wall/lj126 zhi EDGE $16.0 \quad 0.5 \quad 0.56123$

$\begin{array}{lrllll}\text { \# } 10 \quad \mathrm{~L}-\mathrm{J} & \mathrm{R} L & \mathrm{eps} & \mathrm{sig} & \mathrm{cut} & \mathrm{x}(0.64 / 0.5236)^{\wedge} 1 / 3 \\ \text { pair_coeff } & 1 & 1 & 1.0 & 0.213841 & 0.400275 \\ \text { pair_coeff } & 1 & 2 & 10.0 & 0.641522 & 0.720083 \\ \text { pair_coeff } & 2 & 2 & 10.0 & 1.069203 & 1.200138 \\ \text { run } 10 \odot & & & & & \end{array}$


$\begin{array}{lllllll}\text { fix } 3 \text { all wall/lj126 } & \text { xlo EDGE } & 18 . \odot & \odot .5 & 0.56123 \\ \text { fix } 4 \text { all wall/lj126 } & \text { xhi EDGE } & 18 . \odot & \odot .5 & 0.56123 \\ \text { fix } 5 \text { all wall/lj126 ylo EDGE } & 18 . \odot & 0.5 & 0.56123 \\ \text { fix } 6 \text { all wall/lj126 yhi EDGE } & 18 . \odot & \odot .5 & 0.56123 \\ \text { fix } 7 \text { all wall/lj126 zlo EDGE } & 18 . \odot & 0.5 & 0.56123 \\ \text { fix } 8 \text { all wall/lj126 zhi EDGE } & 18 . \odot & 0.5 & 0.56123\end{array}$

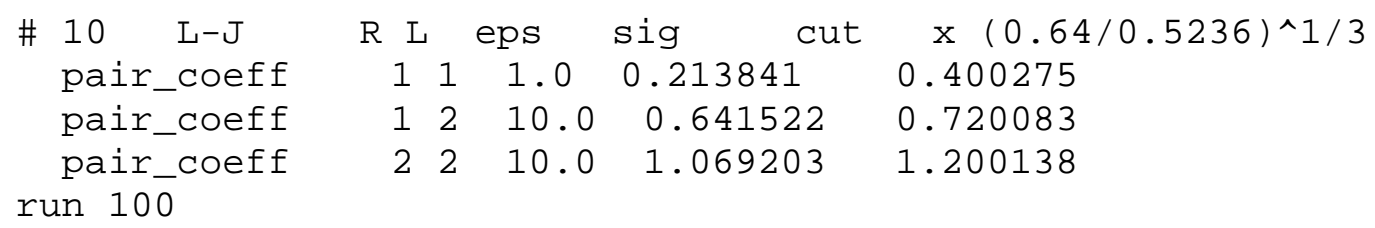

fix 3 all wall/lj126 xlo EDGE $19.0 \quad 0.5 \quad 0.56123$

fix 4 all wall/lj126 xhi EDGE $19.0 \quad 0.5 \quad 0.56123$

fix 5 all wall/lj126 ylo EDGE $19.0 \quad 0.5 \quad 0.56123$

fix 6 all wall/lj126 yhi EDGE $19.0 \quad 0.5 \quad 0.56123$

fix 7 all wall/lj126 zlo EDGE $19.0 \quad 0.5 \quad 0.56123$

fix 8 all wall/lj126 zhi EDGE $19 . \odot \quad 0.5 \quad 0.56123$
\# $10 \mathrm{~L}-\mathrm{J} \quad \mathrm{R} \mathrm{L}$ eps sig cut $\times(0.64 / 0.5236)^{\wedge} 1 / 3$
$\begin{array}{lllll}\text { pair_coeff } & 11 & 1.0 & 0.213841 & 0.400275\end{array}$
$\begin{array}{lllll}\text { pair_coeff } & 12 & 10.0 & 0.641522 & 0.720083\end{array}$

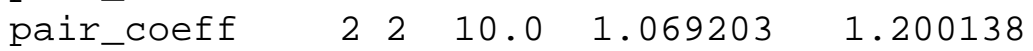
run 100

fix 3 all wall/lj126 xlo EDGE $20.0 \quad 0.5 \quad 0.56123$

fix 4 all wall/lj126 xhi EDGE $20.0 \quad 0.5 \quad 0.56123$

fix 5 all wall/lj126 ylo EDGE $20.0 \quad 0.5 \quad 0.56123$

fix 6 all wall/lj126 yhi EDGE $20.0 \quad 0.5 \quad 0.56123$

fix 7 all wall/lj126 zlo EDGE $20.0 \quad 0.5 \quad 0.56123$

fix 8 all wall/lj126 zhi EDGE $20.0 \quad 0.5 \quad 0.56123$
\# $10 \quad \mathrm{~L}-\mathrm{J}$
$R$ L eps sig
cut
$\times(0.64 / 0.5236)^{\wedge} 1 / 3$
pair_coeff
pair_coeff
$\begin{array}{lllll}1 & 1 & 1.0 & 0.213841 & 0.400275\end{array}$
$\begin{array}{llll}1 & 2 & 10.0 & 0.641522\end{array}$
0.720083
pair_coeff
$\begin{array}{llll}2 & 2 & 10.0 & 1.069203\end{array}$
1. 200138

run 100 